\title{
Device Applications of Solution Processed MIR Semiconductor Nanocrystal Thin Films
}

by

\author{
Matthew Cryer
}

A Thesis

submitted to the Victoria University of Wellington in fulfilment of the requirements for the degree of Doctor of Philosophy in Physics.

Victoria University of Wellington 2019 

'You have to think like a mountain climber.' 'Oh, do I?' 'Yes. Some people take days, sweat buckets, endure pain and cold and risk injury and - in some cases - permanent death to achieve the summit of a mountain only to discover there a party of their peers freshly arrived by aircraft and enjoying a light picnic.' 'If I was one of those climbers I'd be pretty damned annoyed.' 'Well, it is considered rather impolite to land an aircraft on a summit which people are at that moment struggling up to the hard way, but it can and does happen. Good manners indicate that the picnic ought to be shared and that those who arrived by aircraft express awe and respect for the accomplishment of the climbers.' 'The point, of course, is that the people who spent days and sweated buckets could also have taken an aircraft to the summit if all they'd wanted was to absorb the view. It is the struggle that they crave. The sense of achievement is produced by the route to and from the peak, not by the peak itself. It is just the fold between the pages.' The avatar hesitated. It put its head a little to one side and narrowed its eyes. 'How far do I have to take this analogy, $\mathrm{Cr}$ Ziller?'

Iain M. Banks Look to Windward 


\section{Abstract}

Colloidal semiconductor nanocrystals (NCs) with bandgaps less than $1 \mathrm{eV}$ allow the development of mid wave infrared (MIR) sensitive detectors that exploit the benefits of colloidal materials, primarily bandgap selection and solution deposition. Additionally, the electrical behaviour of these films can be examined for characteristics that can increase the functionality of NC based detectors.

The production of devices that are designed to be competitive as ultralow-cost, room temperature MIR detectors, operating with photonic, rather than thermal detection is detailed. The evolution of the colloidal synthesis, spray deposition methods, substrate materials and post deposition treatments used here lead to highly robust and high performing devices. These devices demonstrate a "colour" sensitivity down to 300 $\mathrm{nm}$ in the $\mathrm{MIR}(\approx 10 \%$ of scale), with superior responsivities for this class of device, up to $0.9 \mathrm{~A} \mathrm{~W}^{-1}$, and competitive specific detectivity up to $8 \times 10^{9}$ Jones at $200 \mathrm{~Hz}$ and $300 \mathrm{~K}$. Furthermore, these devices utilise a cheap and robust substrate material that allows operation after deformation up to $45^{\circ}$ without degradation over many cycles. These devices offer a template for ultra-low-cost MIR detectors with performance that rivals microbolometers but with better measurement speed and spectral sensitivity. As such these devices showcase the key advantages of using colloidal NCs in MIR applications. 
Planar and fully air processed thin film devices that demonstrate photoinduced memristive behaviour and can be used as a transistors, photodetectors or memory devices are investigated. Following long term $(60 \mathrm{~h})$ air exposure, unpackaged NC films develop reliable memristive characteristics in tandem with temperature, gate and photoresponse. On/off ratios of more than 50 are achieved and the devices show long term stability, producing repeatable metrics over days of measurement. The on/off behaviour is shown to be dependent on previous charge flow and carrier density, implying memristive rather than switching behaviour. These observations are described within a long term trap filling model. This work represents an advance in the integration of NC films into electronic devices, which may lead to the development of multi-functional electronic components.

Building on the previous work the steps taken to move from a planar device, that works well in controlled conditions, to a multi-pixel sensor that can demonstrate MIR video imaging at room temperature in a noisy environment are shown. This is achieved with a 15 pixel detector that consists only of a polymer substrate and solution patterned NC pixels. This device can detect a $373 \mathrm{~K}$ object with the device at $298 \mathrm{~K}$ in a noisy environment. This performance is enabled by photogain at $5 \mathrm{~V}$ bias that reaches a maximum External Quantum Efficiency (EQE) of $1940 \pm 290 \%$ for a pixel with a $3.3 \mu \mathrm{m}$ bandgap. Through the use of four separate bandgaps it is shown that "multicolour" thermal imaging systems can deliver another layer of information, on top of intensity, to the user. The behaviour of the system is examined under use and it is shown that the photoconductive device behaves as expected with regards to bias, and that trap enabled gain is sensitive to total incident flux, more than the spectral energy distribution of the target. Finally, it is shown that solution patterned QD fabrication methods can deliver electrical reproducibility between pixels that is sufficient to allow an imaging plane of multiple pixels. 
The somewhat neglected tin chalcogenide semiconductor nanocrystals are investigated and inverse MIR detection at room temperature is demonstrated with planar, solution and air-processed PbSnTe and SnTe QD devices. The detection mechanism is shown to be mediated by an interaction between MIR radiation and the vibrational stretches of adsorbed hydroxyl species at the oxdised NC surface. Devices are shown to possess $\mathrm{mA} \mathrm{W}^{-1}$ responsivity via a reduction in film conductance due to MIR radiation and, unlike classic MIR photoconductors, are unaffected by visible wavelengths. As such these devices offer the possibility of MIR thermal imaging that has an intrinsic solution to the blinding caused by higher energy light sources.

In summary, it is shown that semiconductor NCs with an all ambient fully solution processed deposition and ligand exchange procedure can be used to create simple, robust and cheap devices that are beginning to demonstrate metrics on par with current commercial thermal detector systems. It is also shown that these devices can under certain circumstances demonstrate novel behaviours that offer the prospects of enhanced or novel functionality. 


\section{Acknowledgments}

My greatest and most sincere thanks go to my wife Laura. Thank you for supporting me physically and mentally, you are the love of my life and I am very lucky to have found you.

To my supervisor A/Prof Jon Halpert, thank you for giving me the opportunity to study for my $\mathrm{PhD}$, I appreciate that you took a chance as I could have been very unsuited to research based on the job I was doing when we first spoke. Secondly, thank you for the constant pressure to get results and work towards publication, not only have I produced a body of work of which I am proud, the focus on publication kept me motivated throughout. Thirdly, thank you for trusting me to work independently for the second 18 months after you moved to Hong Kong. I felt that as I got up to speed we made a good team.

To Dr Natalie Plank, thank you for letting me hang around your group, for arranging my defence and for agreeing to be my Victoria Supervisor.

To David Flynn, thank you initially for your teaching and patience and after that for your friendship and support (and coffee).

To Dr Parth Vashishtha thank you for all your help with the chemistry side of things and your constant positivity, it made my first year a lot easier. I will not miss trying to follow you up stairs.

To Dr Holger Fiedler, thank you for your tolerance of talking about work over coffee. Having someone of your experience and honesty to steer my abstract theorising was invaluable.

To (hopefully Dr by now) Leo Browning, thank you for letting me mess 
viii

about with all your new test equipment, but more importantly thank you for your constant chat about "the arts" and mechanical keyboards. Our career brainstorming sessions were pure cathartic gold.

To Felicia Ullstad, Hamish Colenso and Jackson Miller, thank you for keeping me sane during the last 6 months.

To Erin Service, Geoff Laufersky, Karen Thorne, Mohsen Maddah, Selvan MT and everyone else I annoyed while they were trying to work, thank you for your tolerance.

To Nick Grinter, Alex Puglisi and Alan Rennie, it pains me to say it, but you are the unsung heroes of the School. I would not have been able to complete the volume of work I have without your tolerance of my last minute requests, thank you.

To Major David Bunker, thank you for your support in my last year at work. You managed to keep it together even when I was doing calculus in the back of my vehicle when I should have probably been looking after your Company.

To my Aunt, Emeritus Prof Margaret Harris FRS, thank you for the encouragement, "You should get your PhD." was good advice.

Finally, thank you to my parents. Your tireless support and belief in me has enabled everything else. An upbringing where it was considered completely normal behaviour to get the dictionary out over dinner led me to become who I am today. There is no more valuable a gift than the opportunity to think for oneself, and you gave that to me in spades. 


\section{Contents}

1 Introduction and Background 1

1.1 Introduction . . . . . . . . . . . . . . 1

1.2 Fundamentals .................... 2

1.2.1 Blackbody Radiation . . . . . . . . . . . . 3

1.2.2 Principles of Infrared Detection . . . . . . . . . . . 6

1.2.3 Photodetection Concepts . . . . . . . . . 8

1.2.4 Transistors .................. 12

1.2 .5 Memory . . . . . . . . . . . . . . 15

1.2.6 Carrier Generation . . . . . . . . . . . . . 16

1.2 .7 Density of States . . . . . . . . . . . . . 18

1.2.8 Conduction in QD Films . . . . . . . . . . . . . . . 20

1.3 Current Detector Technology . . . . . . . . . . . . 27

1.3.1 History . . . . . . . . . . . . . 27

1.3.2 Thermal Detectors . . . . . . . . . . . . . 28

1.3.3 Bulk Semiconductors . . . . . . . . . . . . . 28

1.3.4 Detector Characteristics . . . . . . . . . . . . 30

1.3.5 Detector Noise . . . . . . . . . . . . . . 32

1.4 Quantum Dots . . . . . . . . . . . . . . . . . 33

1.4.1 Theoretical ................. 33

1.4.2 Colloidal QD Synthesis . . . . . . . . . . 36

1.5 Quantum Dot Infrared Photo Detectors (QDIPS) . . . . . . . 41

1.5.1 Quantum Dot use in Detectors . . . . . . . . . . . . 42 
1.5.2 Current QDIP Devices . . . . . . . . . . . . . . 46

1.6 Summary . . . . . . . . . . . . . . . . 50

2 Methods 53

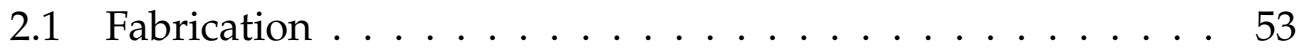

2.1 .1 QD Synthesis . . . . . . . . . . . . . . . . 53

2.1.2 Photolithography . . . . . . . . . . . . . . . . 55

2.1.3 Device Solution Processing . . . . . . . . . . . . 57

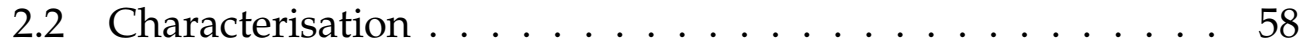

2.2.1 IR Absorbance Measurements . . . . . . . . . . . 58

2.2.2 Transmission Electron Microscopy . . . . . . . . . . . 59

2.2.3 Scanning Electron Microscopy . . . . . . . . . . . 62

2.2.4 X-ray Diffraction . . . . . . . . . . . . . . . 63

2.2.5 Parameter Analyser . . . . . . . . . . . . . . . 64

2.2.6 Opto-Electrical Measurements . . . . . . . . . . . 65

2.2.7 Multi Channel Measurement System . . . . . . . . . . 70

3 HgTe Synthesis and MIR Detection $\quad 75$

3.1 Motivation . . . . . . . . . . . . . . . . . 75

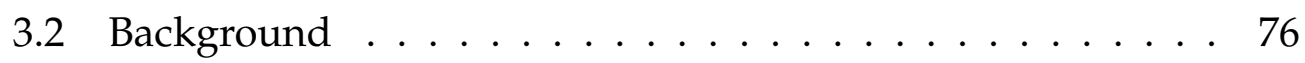

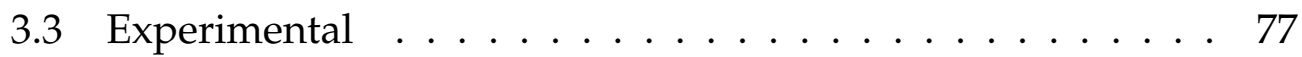

3.3.1 CQD Synthesis . . . . . . . . . . . . . . . . . . 77

3.3.2 CQD Purification . . . . . . . . . . . . . . . . . . 79

3.3.3 Device Design and Fabrication . . . . . . . . . . . 79

3.3.4 Film Deposition Methods . . . . . . . . . . . . . 82

3.3.5 Characterisation . . . . . . . . . . . . . . 84

3.4 Results . . . . . . . . . . . . . . . 86

3.4.1 QD Characterisation . . . . . . . . . . . . 86

3.4.2 Organic Ligands on Silicon Substrates . . . . . . . . 88

3.4.3 MIR Photodetection with Silicon Substrates . . . . . . 93

3.4.4 Post Deposition Inorganic Ligand Exchange . . . . . 94

3.4 .5 Kapton Substrates . . . . . . . . . . . . . . . . . . 98 
3.4.6 PET Substrates . . . . . . . . . . . . . . . . . . . . 100

3.4.7 Room Temp Flexible Device . . . . . . . . . . . . . 101

3.5 Discussion . . . . . . . . . . . . . . . . . . . . . . . . 111

3.6 Conclusion . . . . . . . . . . . . . . . . . . . . . . . 120

4 Memristance in Oxidised HgTe Devices 123

4.1 Motivation . . . . . . . . . . . . . . . . . . . . . . . . . . 123

4.2 Background . . . . . . . . . . . . . . . . . . . . . . . . . 124

4.3 Experimental . . . . . . . . . . . . . . . 126

4.3 .1 Devices . . . . . . . . . . . . . . . . . . 126

4.3.2 Measurement . . . . . . . . . . . . . . . . . 126

4.4 Results . . . . . . . . . . . . . . . . . . . . . . . . . 128

4.4.1 Initial Characterisation . . . . . . . . . . . . . . . 128

4.4 .2 IV Hysteresis . . . . . . . . . . . . . . . . . . . 132

4.4.3 Memristance and the Memory Cycle . . . . . . . . . 135

4.4.4 Time Evolution . . . . . . . . . . . . . . . . . . . . 137

4.4.5 Transistor Behaviour . . . . . . . . . . . . . . . . . . . 139

4.4 .6 Memory . . . . . . . . . . . . . . . . . . . . 142

4.5 Discussion . . . . . . . . . . . . . . . . . . . . . . 148

4.5.1 Hysteresis Dependence on Temperature and Illumination . . . . . . . . . . . . . . 150

4.5.2 Memory Dependence on Temperature and Illumination . . . . . . . . . . . . . . . . 155

4.6 Conclusion . . . . . . . . . . . . . . . . . . . . . . . . . . . 157

5 Multi Pixel Imaging with Photogain Enhanced Detection 161

5.1 Motivation . . . . . . . . . . . . . . . . . . . . 161

5.2 Background . . . . . . . . . . . . . . . . . . . 162

5.3 Experimental . . . . . . . . . . . . . . . . . . . . . 163

5.3.1 Device Design . . . . . . . . . . . . . . . . . . . . . 164

5.3 .2 Silicon Devices . . . . . . . . . . . . . . . . . . 165

5.3.3 PMMA Devices . . . . . . . . . . . . . . . . . . . . 166 
5.4 Results . . . . . . . . . . . . . . . . . . . . . 170

5.4 .1 Multicolour Imaging . . . . . . . . . . . . . . . 173

5.5 Discussion . . . . . . . . . . . . . . . . . 176

5.6 Conclusion . . . . . . . . . . . . . . . . . . . . . . 182

6 Tin Chalcogenide Quantum Dots 185

6.1 Motivation . . . . . . . . . . . . . . . . . . . . 185

6.2 Background . . . . . . . . . . . . . . . 186

6.3 Experimental . . . . . . . . . . . . . . . . . . . . . 187

6.4 Results . . . . . . . . . . . . . . . . . . . . . 190

6.4 .1 XRD and Absorbance . . . . . . . . . . . . . 190

6.4.2 QD Characterisation . . . . . . . . . . . . . . 195

6.4.3 Device Characterisation . . . . . . . . . . . . . . 205

6.4 .4 MIR Optical Response . . . . . . . . . . . . . . . . 206

6.5 Discussion . . . . . . . . . . . . . . . . . . . . . 210

6.5 .1 Absorbance Features . . . . . . . . . . . . . . . . 210

6.5 .2 Electrical Response . . . . . . . . . . . . . . . . 216

6.6 Conclusion . . . . . . . . . . . . . . . . . . . . . . . . . . . . . 219

7 Conclusion and Further Work 223

7.1 Conclusion . . . . . . . . . . . . . . . . . . . . 223

7.1.1 HgTe Quantum Dots for MIR Imaging . . . . . . . . . 223

7.1.2 Memory Applications . . . . . . . . . . . . . . 226

7.1.3 Tin based Quantum Dots for Sensing _ . . . . . . 226

7.2 Further Work . . . . . . . . . . . . . . . . . . . 227

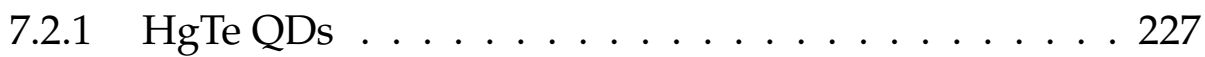

7.2.2 Ambipolar Transistor Behaviour . . . . . . . . . . 228

7.2 .3 Memory Behaviour . . . . . . . . . . . . . . . . . . . 229

$7.2 .4 \quad$ SnTe Devices . . . . . . . . . . . . . . . . . . . 229

7.3 Summary . . . . . . . . . . . . . . 230

$\begin{array}{ll}\text { A Acronyms } & 251\end{array}$ 
B Why Use Multiple Band Gaps? 


\section{Chapter 1}

\section{Introduction and Background}

\subsection{Introduction}

Due to the high cost of commercial detectors the infrared (IR) portion of the electromagnetic spectrum is underused. Because the military and aerospace industries can afford the current technology there has been no motivation to remove the underlying cost driver [1,2]. Namely vacuum insulated and/or cryogenically cooled detector elements. This high cost has severely limited the wider uptake of the technology [3,4]. There are numerous known applications for IR imaging, including, but not limited to, military field imaging at all scales, aerospace fault detection, standoff chemical detection, communications, passive observation and medical imaging $[5,6]$. Yet if the technology can undergo a large reduction in cost or increase in miniaturisation then the potential number of applications is large, a few examples being:

1. Compact chemical identification systems that miniaturise the principles of IR spectroscopy for use in sniffer and search devices [7].

2. Collision detection and imaging systems that are not affected by ambient particulate or light conditions [8,9].

3. Industrial process control and tagging technology. [7]. 
4. Portable and discreet biometric identification [10].

5. Active and passive surveillance systems with increased functionality [11].

6. Targeted environmental and heat loss monitoring to enhance energy efficiency [12].

The wider uptake of MIR imaging technology is not dependent upon finding uses for the technology, but on the current device architecture that limits application due to its bulk, weight, cost, complexity, and limited field of view [1,2]. The problem that this thesis will address is, can quantum dots (QDs) be used to make simple and cheap techniques to produce devices that solve known problems with current technology?

Due to the highly multidisciplinary nature of this work the breadth of background knowledge is large. The remainder of this Chapter aims to provide the basic knowledge and literature review that is required to understand the method and intent of the results Chapters. Any highly specific background will be found in the Chapter in which it is required.

\subsection{Fundamentals}

Interest in the IR is driven by a motivation for a remote imaging method that does not require an external energy source, in the case of thermal IR utilising the blackbody emission of the target object. This ability to passively detect is the reason why the IR is commonly synonymous with "night vision". This work is concerned with thermal night vision, not with image intensification night vision, the latter using low levels of reflected ambient radiation to image a target, not emitted radiation. Successful imaging requires high transmittance through the medium existing between the detector and the target, usually air, therefore detectors must 
be operated in an atmospheric spectral window. The IR spectrum is split into regions that correspond to windows in atmospheric absorbance, this is shown in Figure 1.1. The defined regimes of IR radiation are [14]:

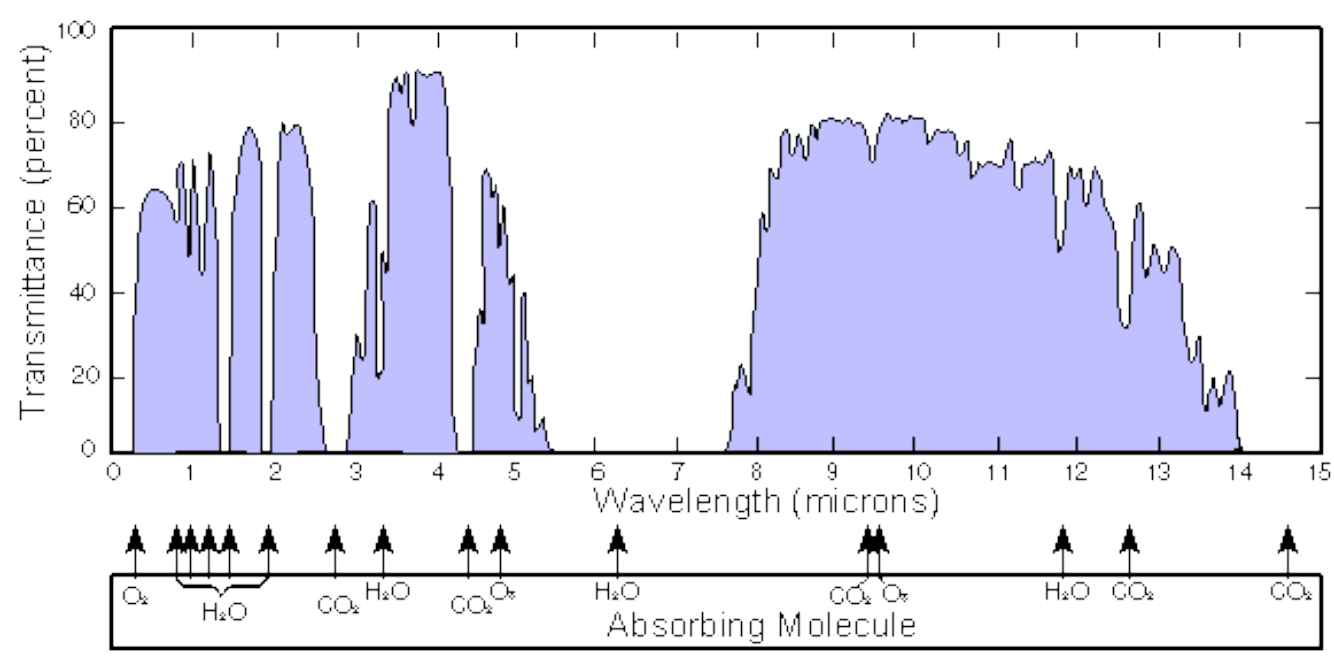

Figure 1.1: Atmospheric transmittance windows and the molecular vibrations that cause absorbance. Reproduced under Creative Commons-ShareAlike License [13].

- Near-IR (NIR) from 0.7 to $1 \mu \mathrm{m}$.

- Short-wave IR (SWIR) from 1 to $3 \mu \mathrm{m}$.

- Mid-wave IR (MIR) from 3 to $5 \mu \mathrm{m}$.

- Long-wave IR (LWIR) from 7 to $14 \mu \mathrm{m}$.

This work is concerned with the integration of nanomaterials that demonstrate an onset of absorption somewhere between 2 and $5 \mu \mathrm{m}$, into photodetectors.

\subsubsection{Blackbody Radiation}

The underlying physics of the electromagnetic radiation emitted by bodies of a defined temperature was discovered and quantified by Max Planck in 
1914 [15]. The most common form of this is the equation showing spectral distribution with wavelength $\left(B_{\lambda}(\lambda, T)\right)$,

$$
B_{\lambda}(\lambda, T)=\frac{2 h c^{2}}{\lambda^{5}} \frac{1}{e^{\frac{h c}{\lambda k T}}-1} .
$$

Where $h$ is the Planck constant, $c$ is the speed of light, $\lambda$ is the wavelength and $k$ is the Boltzmann constant. When considering infrared detection the underlying blackbody emission is best depicted by spectral emission curves that are defined by the temperature of the object that is the detection target. Figure 1.2 shows that we can easily identify possible temperature detection regimes if we know the wavelengths of light that can be detected by that specific detector/material. For example, a detector that can detect light of $4 \mu \mathrm{m}$ will never be able to see a body at $200 \mathrm{~K}$, however it could well see a body at $300 \mathrm{~K}$ if it is sufficiently sensitive. 


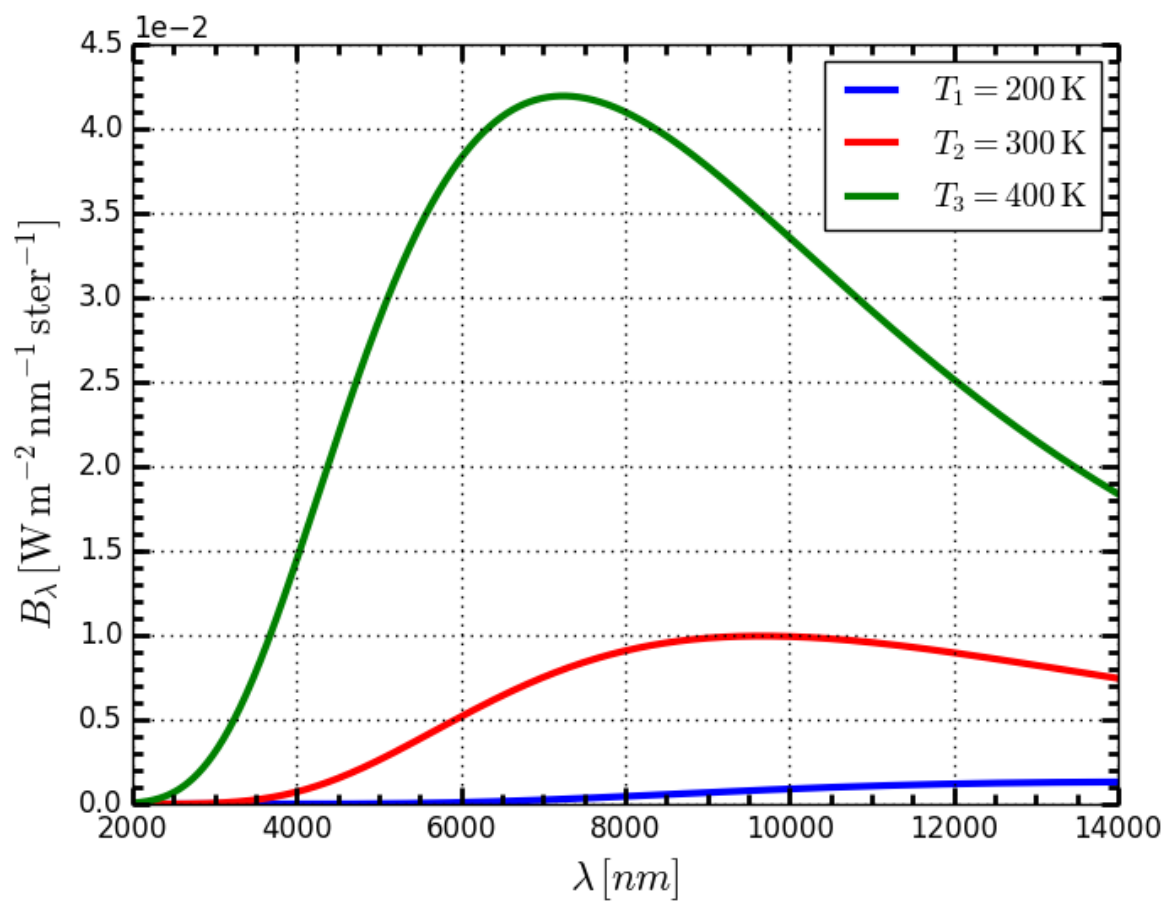

Figure 1.2: Planck curves showing idealised blackbody spectral radiance against wavelength for three different temperatures. Note that for room temperature objects the total flux is much greater at $8 \mu \mathrm{m}$ than at $3 \mu \mathrm{m}$, hence the common use of LWIR detectors. Note also that the relative difference between $300 \mathrm{~K}$ and $400 \mathrm{~K}$ is greater in the MIR than the LWIR.

The MIR region is conventionally defined as the region that spans the peak emission for bodies with a temperature between $\approx 1500 \mathrm{~K}$ and 600 $\mathrm{K}(2-5 \mu \mathrm{m})$. The peak emission for a body at $300 \mathrm{~K}$, body temperature, occurs at $9.65 \mu \mathrm{m}$. Up until now it has been necessary to reduce the band gap energy of the sensitiser material beyond $8 \mu \mathrm{m}$ and into the Long Wavelength IR (LWIR) in order to detect a $300 \mathrm{~K}$ body. This reasoning however, is only correct when considering the wavelength of peak emission given by Wien's Displacement Law [16],

$$
\lambda_{\text {peak }} T=2.898 \times 10^{-3} \mathrm{~m} \mathrm{~K} .
$$


In reality (as shown in Figure 1.2) a body at $300 \mathrm{~K}$ emits non-negligible radiation at wavelengths down to $3 \mu \mathrm{m}$. An alternate definition is that the MIR contains the emission onset of objects that are at $300 \mathrm{~K}$ and above. Figure 1.2 shows that the total photon flux for a body at $300 \mathrm{~K}$ is higher at $8 \mu \mathrm{m}$ than at $5 \mu \mathrm{m}$. This makes it considerably easier to detect a $300 \mathrm{~K}$ body with an $8 \mu \mathrm{m}$ detector in the LWIR, than in the MIR. This however comes at the cost of target temperature sensitivity. As a worked example, the ratio in photon flux between a $300 \mathrm{~K}$ body and a $200 \mathrm{~K}$ body at $8 \mu \mathrm{m}$ is on the order of 10:1. At $5 \mu \mathrm{m}$ it is much higher, on the order of 100:1. Considering the implication of this for spectral selection at the detector, it is clear that to see small differences in temperatures above $300 \mathrm{~K}$ one wants to use a detector that has spectral onset of absorption in the MIR. It should be made clear that to use this theoretical difference in ratio requires a sufficiently sensitive MIR sensor as the MIR flux at these temperatures is considerably lower than the LWIR flux. Currently, due to the use of single band gap detectors this "colour" sensitivity remains a hypothetical problem rather than a performance limiter. As single bandgap (single "colour") detectors only need function as intensity detectors. This is due to higher photon emission in the LWIR region, and as such single colour detection in the $300 \mathrm{~K}$ target range is far easier in the LWIR than the MIR [14,17].

\subsubsection{Principles of Infrared Detection}

The general model for a detector can be seen in Figure 1.3 [17]. In this model the window provides isolation from the ambient environment and as such it selects the environment (i.e. vacuum, nitrogen etc) and spectrum of light that is ultimately incident on the transducer. The aperture selects the field of view, becoming very important when considering multi pixel sensors. The transducer is the sensor array that converts radiation into electrical power and finally the readout electronics amplify and process the transducer output into an electrical signal that is compatible with the 


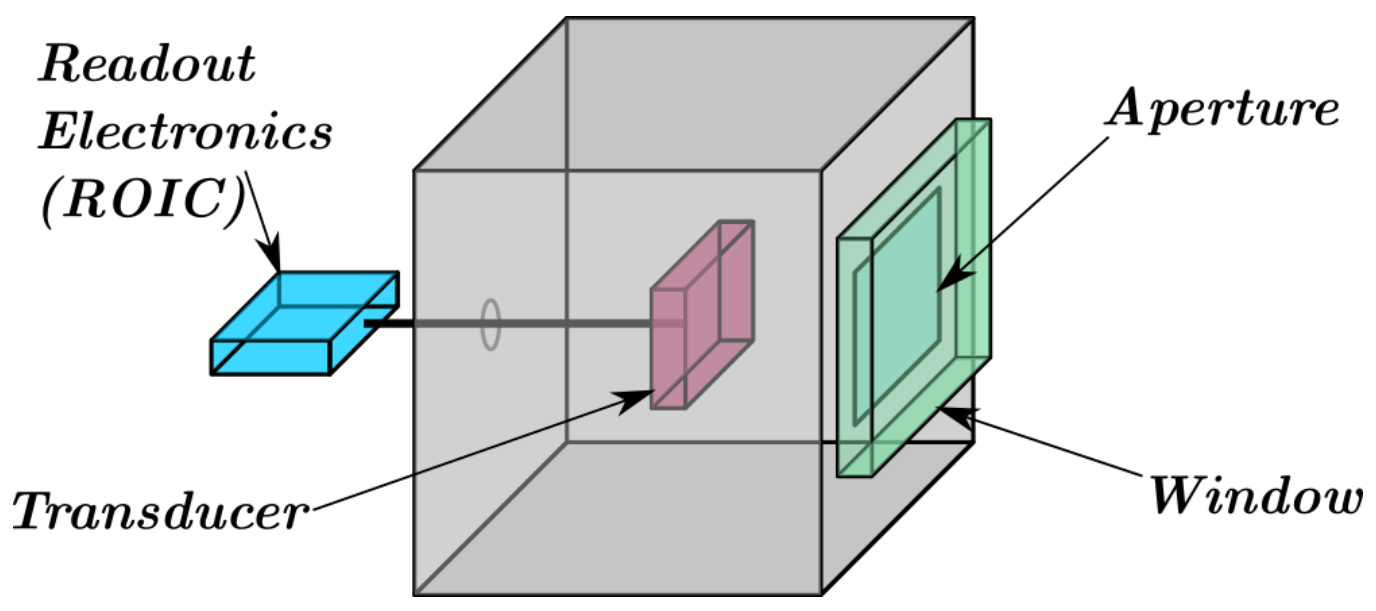

Figure 1.3: A simple cartoon view showing the generic components of a detector system, this work is focussed on the transducer.

intended use [17]. This thesis is concerned with the integration of nanomaterials into the transducer, which shall be referred to as "the detector". Chapter 5 will however touch on the purpose and use of transducer side signal processing with regard to the signals received from these new detector prototypes. Aperture and window considerations will be used to test the behaviour of the materials, but will not be discussed from a holistic device engineering perspective.

There are three key input signal variables that define the behaviour of a semiconductor based photon detector. They are [18]:

1. The intrinsic thermal carrier density $\left(n_{i}\right)$ [19]:

$$
n_{i}=N_{C} N_{V} e^{\frac{-E g}{2 k T}}
$$

where $N_{C}$ and $N_{V}$ are the effective density of states in the conduction and valence bands respectively and $E_{g}$ is the bandgap. The dark current due to these thermal carriers is denoted as $I_{0}$, which should be constant for a given temperature. The noise in the dark signal is denoted $\sqrt{I_{0}}[18]$. 
2. The background photon flux incident on the detector, $\Phi_{B}$. This is the total number of photons incident from everything that is not the intended target. The current due to the background flux is denoted as $I_{b k g d}$.

3. The signal flux, $\Phi_{S}$ and the signal current $I_{\text {signal }}$.

The general conditions for detection can be expressed in terms of these variables. If the dark current noise is greater than the background current or signal current then the detector will produce only a noise signal. By cooling the detector the number of thermal carriers, and hence also the dark current, can be greatly reduced and this is the reason that current photonic IR detectors are cooled [4,20]. To see an object against a background (noting that the background flux is not solely the conventional image background but also the incident photons from the device structure itself) the signal current must be greater than the total background current, and this difference must be greater than the dark current noise [21], simply defined by

$$
I_{\text {signal }}>I_{b k g d}+\sqrt{I_{0}} .
$$

It can also be seen from equation 1.3 that there is an inverse relationship between the bandgap and the noise signal at any given detector temperature $(T)$. This is another reason why MIR detectors, which operate with larger bandgap energies than LWIR detectors, are a more desirable option for the development of uncooled devices.

\subsubsection{Photodetection Concepts}

There are many different types of IR detector. The first division in these is between photonic/quantum detectors and thermal detectors $[4,20]$. The working principal of thermal detectors is exploiting the changing conductivity of the material with temperature $[4,20]$. Quantum detectors all share the fact that photons are absorbed by the material and the absorption of 
above bandgap photons creates electron hole pairs (EHPs) within the material. The last remaining key division is between photoconductive (PC) and photovoltaic (PV) operation. PC detectors absorb photons generating free charge carriers and thus have an observably increased conductivity $[20,22]$. They are operated under a non-zero bias voltage and the changing resistance in the circuit with illumination allows an output signal to be observed. PC detectors do not require a built in field to operate and as such are the simplest type of device design by far [17], shown in Figure 1.4.

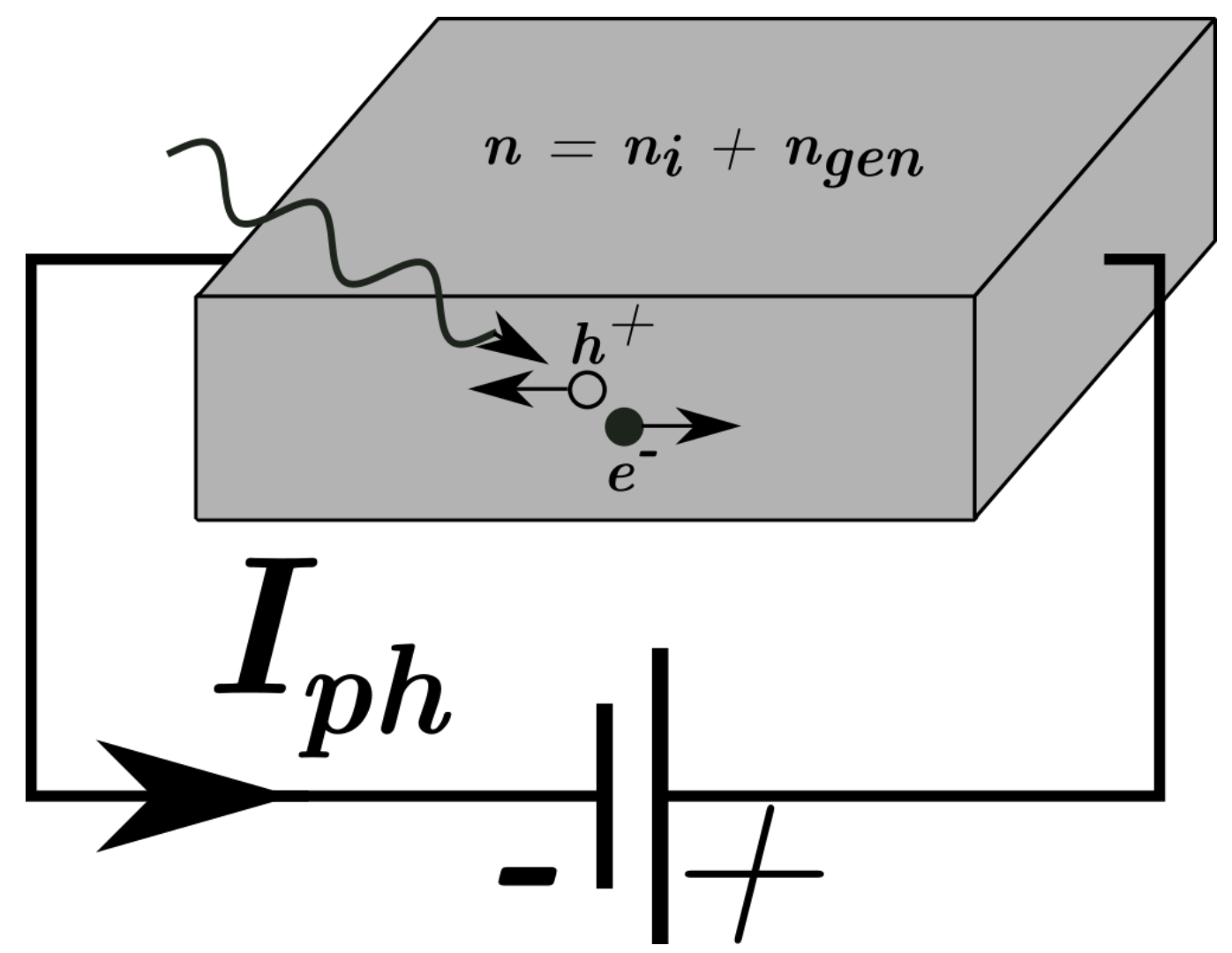

Figure 1.4: Diagram of a simple PC detector, the photogenerated carriers cause an increase in total carrier density. Under a non zero applied bias this manifests as an increased current or equally a reduction in measured resistance. In this simplified example the dark current is determined by the intrinsic carrier density $\left(n_{i}\right)$. 
In PC devices it is possible to have an EQE greater than unity. This effect is called gain and represents more than one electron flowing per absorbed photon. For gain to occur both contacts to the material must not limit current flow for either carrier, contacts that do not present an energetic barrier to carriers entering or exiting the material are called ohmic contacts [22]. Assuming that the device has ohmic contacts then PC gain will occur if the transit time for holes is different to electrons. If the hole transit time is much slower than the electron transit time then in order for the material to remain electrically neutral, multiple electrons will cycle through the circuit in the time it takes one hole to reach the negative electrode [22]. The increased transit time for one of the carriers can be caused by such factors as a difference in mobility or a differing trapping rate between the carriers.

PV operation is the generation of a short circuit current under no applied bias, the generation of EHPs within the material causing a detectable current without the need for an external electric field [22]. PC detectors are structurally far simpler than PV as there is no need for a suitable structure that allows the generation of a built in field. The most common construction that generates a built in field is the PN junction, shown in Figure 1.5. These are made by implanting electron acceptor ions (p-type) or electron donor ions (n-type) into an intrinsic semiconductor. In the p-type material holes are the majority carrier and in the n-type electrons are the majority carrier. When these two materials are put in physical contact the holes diffuse into the n-type material and recombine and as this also happens with electron diffusion, a physical region that is depleted of carriers is formed. The implanted ions however are physically fixed and therefore there is a region of negative ions near the $\mathrm{p}$-type material and a region of positive ions near the n-type material. It is the field that this charge distribution generates that is the built in field. When non implanted ions in this region absorb a photon an EHP is generated and the electron and hole drift under the influence of the field in different directions, generating a photocurrent. 
Commonly bulk PV photodetectors work in one of two modes, PV or pho-

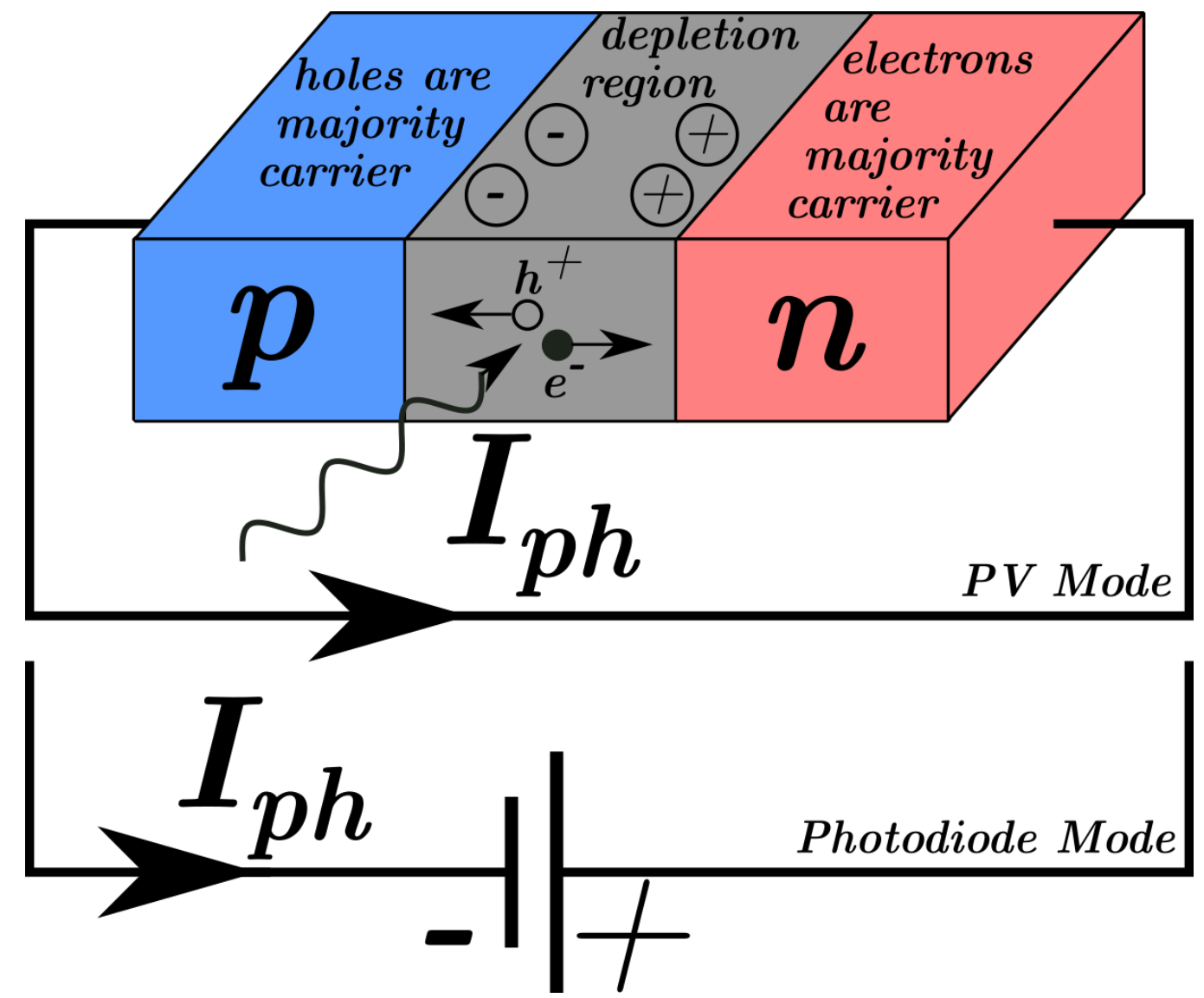

Figure 1.5: Diagram of a PN junction detector, operated in either the PV or the photodiode mode. In the $p$-doped area holes are the majority carrier and in the n-doped area electrons are the majority carrier, the depletion region where these regions meet is where the built in field is created. In the photodiode mode the applied field increases the built in field.

todiode. If there is no applied bias across the junction then the device is working in the PV mode and the incident light will cause a photocurrent $I_{p h}$ across the junction. The use of a load resistance will be required to give a load line, which can be calibrated to incident light power. If the junction is reverse biased it is operating as a photodiode and will still see a change in current with incident radiation. The terminology for reverse bias here means that the applied bias acts to increase the built in field. This reverse 
bias causes a greater accelerating field over the depletion layer, giving a faster response time but the greater current in the device also causes an increase in noise [20,23]. For photodiodes in reverse bias the maximum possible EQE is unity. Gain is not possible because one photon only generates one EHP and each of the carriers recombines when it reaches the edge of the depletion region, independent of the other carrier.

Bulk semiconductor crystals for both PC and PV devices can be grown by a variety of methods, with Molecular Beam Epitaxy (MBE) being the route to the highest performing detectors at this time [24]. This method delivers a high quality crystal over a large area with great control. This method however, is expensive and limited to deposition of one composition of bulk crystal per layer [25], this highlights one of the key design constraints with all IR detectors. One type of semiconductor crystal or PN junction can only have one bandgap energy, therefore bulk crystals can only function as single threshold detectors. To create a two colour detector, where the transducer, rather than the window gives spectral selectivity, requires the use of two unique material compositions. Both must have different lattice constants and material parameters, doubling the cost and severely increasing the complexity of device design [9].

\subsubsection{Transistors}

This section is not intended to be a thorough discussion of a large field, merely sufficient to provide context to the work reported here. The terminology and explanations used here are based on generic organic (OFET)/Thin Film Transistors (TFT) rather than doped bulk semiconductors [26], as these devices most closely identify with nanocrystal transistors [27]. Field Effect Transistors (FETs) are 3-terminal devices where the material under investigation is in electrical contact with the source and drain terminals but is separated from the gate terminal by a thin layer $(<500 \mathrm{~nm})$ of high resistance dielectric which prevents charge 
movement. The material to be investigated that fills the channel between the electrodes has a drain to source electrode length (L) and the perpendicular direction (top down) is called the width (W). Voltage is applied to the drain $\left(V_{d}\right)$ and gate $\left(V_{g}\right)$ electrodes with the source electrode grounded $\left(V_{s}=0\right)$. If this convention is kept then the drain to source voltage $V_{d s}$ is interchangeable with $V_{d}$. If a positive charge is applied to the gate, with no drain voltage applied, electrons will accumulate at the channel/dielectric interface, creating a uniform channel of accumulated charges between the drain and source. In a physically realistic system there will be some distribution of trap states that must be filled before the free carriers in the channel are mobile. The required gate voltage to overcome the trap distribution in a device is called the threshold voltage $\left(V_{T h}\right)$. The bias-stress effect that is observed in Chapter 4 can be interpreted as a re-arrangement of these trap states in energy (or arguably, physically) as a gate voltage is applied, creating a time dependent $V_{T h}$. When a small $V_{d}$ is applied the uniform charge density in the channel changes to a linear gradient of charge density as carriers are being injected at one electrode and extracted at another. This is the linear regime so called because the current is directly proportional to $V_{d}$, shown in Figure $1.6 \mathrm{~b}$. At some $V_{d}$ $=V_{g}-V_{T h}$ the local potential at the source electrode is now less than the threshold voltage and as such there is no longer an open channel; it has been "pinched off" (Figure $1.6 \mathrm{c}$ ). The current that reaches the source is now dependent upon carrier movement between the pinch off point and the electrode, through a depletion region (i.e. depleted of free carriers). If $V_{d}$ continues to increase, the physical position of the pinch off will move towards the drain, but the potential at that point remains $V_{g}-V_{T h}$. Therefore the field driving the conduction through the depletion region remains approximately the same and a saturation of $I_{d}$ is observed (Figure $1.6 \mathrm{~d}$ ). The carrier mobility $(\mu)$ can be extracted in the saturation regime using $[19,26]$,

$$
I_{d}=\frac{W}{2 L} \mu C_{g}\left(V_{g}-V_{T h}\right)^{2}
$$


a)

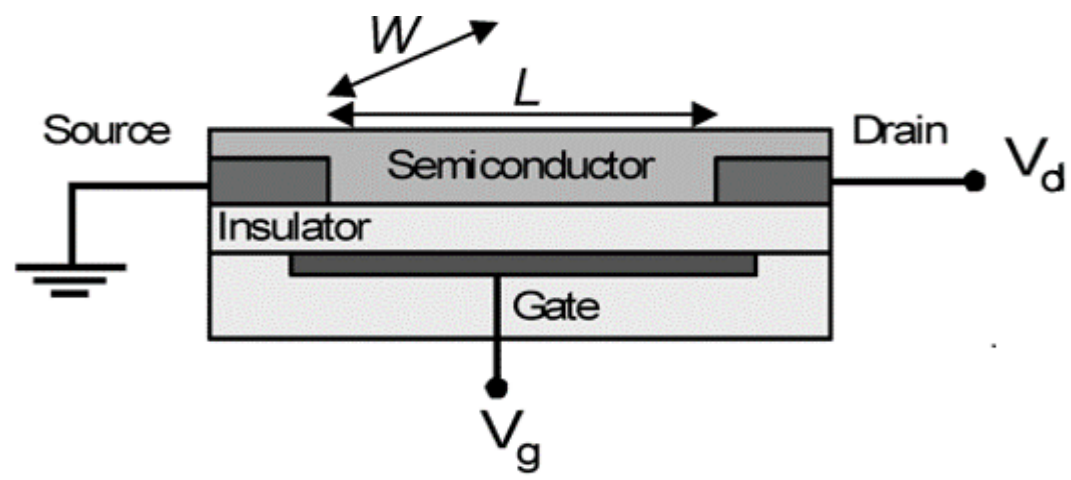

b)
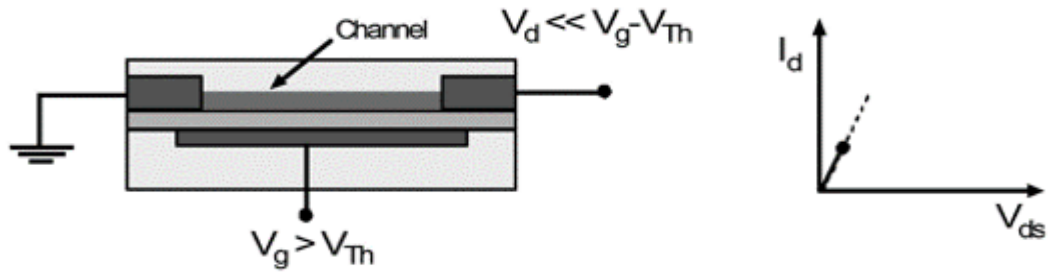

c)
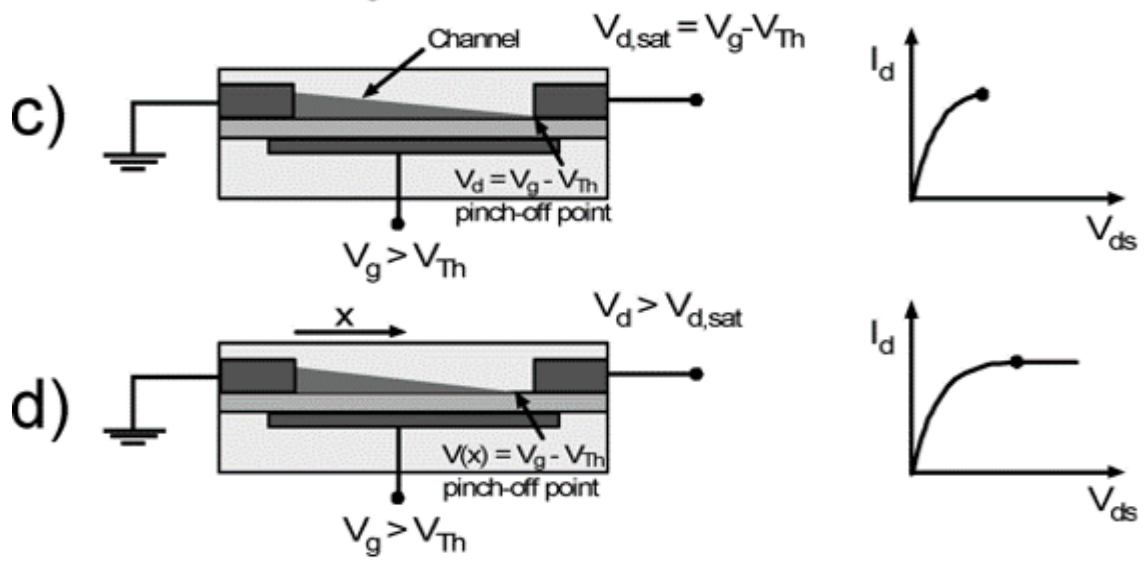

Figure 1.6: $A$, Schematic structure of a field-effect transistor and applied voltages: $L=$ channel length; $W=$ channel width; $V_{d}=$ drain voltage; $V_{g}=$ gate voltage; $V_{T h}=$ threshold voltage; $I_{d}=$ drain current. Illustrations of operating regimes of field-effect transistors: $B$, linear regime. $C$, start of saturation regime at pinch-off. $D$, saturation regime and corresponding current-voltage characteristics. Reprinted unadapted with permission from, Electron and Ambipolar Transport in Organic Field-Effect Transistors, Jana Zaumseil and and Henning Sirringhaus, Chemical Reviews 2007107 (4), 1296-1323. DOI: 10.1021/cr0501543. Copyright 2018 American Chemical Society [26].

Where $C_{g}$ is the capacitance per unit area of the gate dielectric. These linear and saturation regimes are valid as long as there is only one mobile carrier 
in the device. Materials that show gateable behaviour of both electrons and holes demonstrate additional characteristics. This is best envisaged as there being both an electron channel and a hole channel within the device, extending from opposite electrodes. The extent of each channel is determined by such factors as the ratio of the specific carrier mobility to applied voltages, the trap filling in the channel for each carrier and the carrier density. It is a complex system. These systems are best identified by the existence of linear and saturation regimes for both $\mathrm{p}$ and $\mathrm{n}$-type behaviour, and, a third superlinear regime which occurs after the saturation regime if $V_{d}$ continues to be increased. In this regime the channels are directly in contact so there is easy recombination between the carriers causing an increasing $I_{d}$ with $V_{d}$. The saturation regime still exists in both quadrants, because there is a range of $V_{d}$ and $V_{g}$ for which there is a depletion region between the two channels. In this ambipolar case the physical location of the "pinch-off" is not at an electrode but at some point between the two channels [26]. The Author would highlight Figure 12 of reference [26] as a good initial resource for further reading on this topic.

\subsubsection{Memory}

Chapter 4 investigates the use of pure QD films as memory elements. As such a very brief summation of memory technology is included. Electrical memory is defined as a material that has at least two metastable states. The states must be predictably switchable by the application of some stimulus such that they can be "written", and crucially the states must give sufficiently different electrical response that the state of the memory can be discerned, the "read" [28]. The vast majority of volatile memory, defined as that it does not store data without a power source, is constructed from arrays of etched transistors on silicon, and as such suffers from the same limitations as all CMOS technology $[29,30]$. Memory can be judged on a number of very simple metrics [28]. On/off ratio is the ratio between the 
read signal for the on state and the off state. Larger is better but the repeatability of the value is also important. The read time, write time and erase time are all values that allow comparison, and for which lower values are better. To compete with flash memory it is expected that these times will need to be on the order of $\mu \mathrm{s}$ [28]. The retention time is the length of time that the information is held. RAM requires a refresh every few ms, DDR2 DRAM is refreshed every $64 \mathrm{~ms}$ [28]. The longer the retention time the better as this will reduce the power requirement for the device. Cycle endurance is the number of times that the memory element can be rewritten. A typical hard disk drive can be rewritten at least $10^{12}$ times whereas flash can only be rewritten $10^{6}$ times [28]. Any improvement in any of these metrics, or fundamental research that shows a novel material demonstrating memory behaviour is therefore of interest. To date QDs have been used as a replacement for the bulk charge trapping layer within a transistor structure, in part because they can offer more efficient charge trapping [29], but there has been very limited research into QD films acting as memory elements without a transistor structure. This idea of a 2-terminal memory element, rather than 3-terminal element implies a resistance that can be controlled by some parameter. If this resistance change has some time dependence then it is has a memory of the control parameter. This is the concept that is investigated at in Chapter 4.

\subsubsection{Carrier Generation}

Any discussion of an optical detector must, in addition to the conductive behaviour, discuss the behaviour of photogenerated carriers. Here generated carriers are electron-hole pairs that have been created through the absorption of a photon of higher energy than the band gap, or the intrinsic thermally generated carrier population. There are three general ways in which the excited carrier can lose energy, shown in Figure $1.7[19,22,31]$.

1. Band to band recombination. The excited electron falls from the 


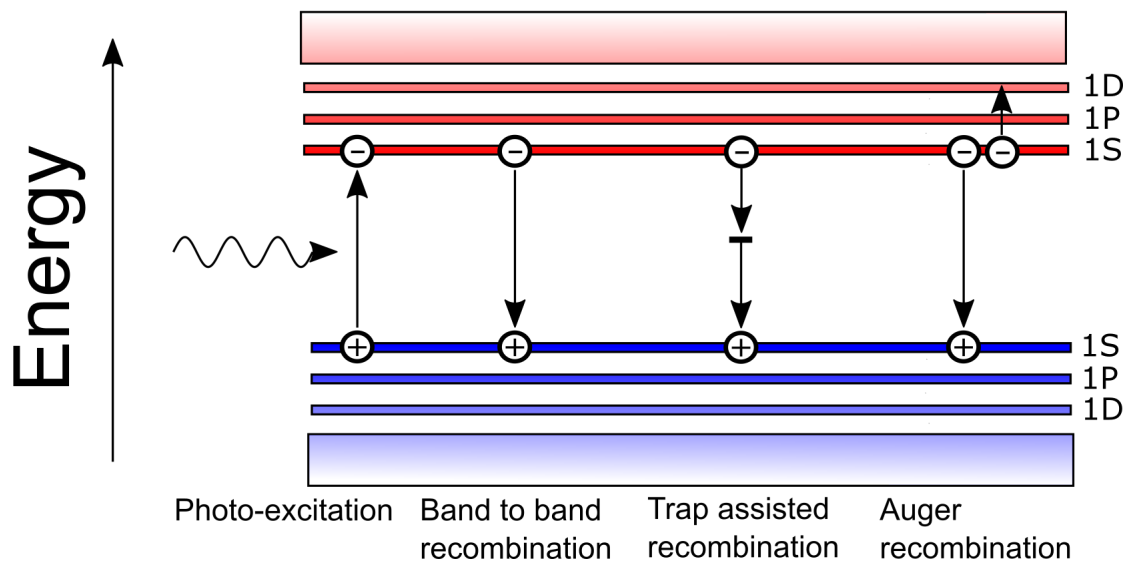

Figure 1.7: Graphical depiction of carrier recombination pathways in a $Q D$ with the first three distinct energy levels shown.

conduction band into the vacant state in the valence band, where it recombines with the hole it created when it was excited. This is a radiative transition where the energy is released as a photon. Band to band recombination can also be non-radiative, where the energy is released as a phonon, more common in indirect bandgap semiconductors.

2. Trap assisted recombination. If there are trap states within the bandgap, for example from an unpassivated surface site, then the excited electron can fall into the trap state. It can then fall again from the intermediate energy trap state into the valence band. Alternatively this can be described as the hole and the electron recombining in the trap site. This could be either a radiative or non-radiative transition.

3. Auger recombination. Electrons can decay by imparting their energy to another electron. This is the Auger effect. In a semiconductor this manifests itself as a transition from a three carrier (two excited electrons, one hole) system to a one carrier system where the remain- 
ing electron has been promoted above the conduction band ground state. This relies on there being a high density of excited carriers and was expected to be suppressed in low degeneracy quantum dot (QD) systems. However recent reports have shown that the high localisation of the electron and hole actually lead to increased Auger rates in some systems [32].

The concept of trap assisted recombination is especially interesting in the framework of QD solids, as unlike in a bulk lattice where the trap sites would be generally distributed throughout as vacancy or interstitial sites, the trap sites on a QD are likely to be more densely located on the surface [32]. If the trap state lifetimes are long compared to the hopping time for conduction band states, there will be a charge distribution that could oppose transport, i.e. the trap state will create an area with a higher charging energy. As such, trap states will negatively affect any device performance by both holding carriers and reducing conduction. Hole traps as well as electron traps are referred to in this work. Hole traps are the donation of an electron to the valence band from a defect site causing a spatially localised positive charge, located here at the surface of a QD. The donated electron fills a hole, which could move, and leaves a localised positive charge, which cannot move and therefore there is a positive charge is trapped at the site of the defect.

\subsubsection{Density of States}

As the result for the 0-D density of states (DOS) will be used below, it is instructive to concisely explain the concept and show the solutions for the 3-D and 0-D situations. In order to calculate carrier concentrations in a material it is vital to know the number of allowed energy levels (states) per unit energy per unit volume in a band. In the most basic treatment of the problem consider a solution where the dispersion relationship is isotropic, i.e. $\mathrm{k}$-space is spherical, and electrons at the bottom of the conduction 
band are treated as free (zero potential in the box) particles, with an effective mass, trapped (infinite potential outside) in a cube. The energy of a trapped conduction band electron is given by [19]

$$
\left(E-E_{C}\right)=\frac{\hbar^{2} k^{2}}{2 m^{*}}
$$

where $E_{C}$ is the energy of the conduction band minimum and $m^{*}$ is the effective mass. When charged particles are moving through a periodic potential, as found in a crystal lattice, the potential acts on the particle so that its resistance to acceleration from an external field is not the same as when the same charged particle is free in vacuum. In order to treat the particle as being free in the crystal, the particle is assigned an effective mass, this quantity encapsulates the different response to an external field imposed by the crystal environment by using a different mass quantity [22]. Taking the potential into account the wavevectors must satisfy, where $n$ is some integer,

$$
k=\frac{\pi n}{L}
$$

in the $\mathrm{x}, \mathrm{y}$ and $\mathrm{z}$ directions. If this is plotted in spherical $\mathrm{k}$-space then the separation between neighbouring k-states is $\frac{\pi}{L}$ in each direction. The reciprocal of the volume of each k-state gives the density of k-states as

$$
\rho_{k}=\frac{L^{3}}{\pi^{3}}=\frac{V}{\pi^{3}}
$$

For any given k-vector the number of available states is the volume of an infinitely thin shell at radius $k$, which has a volume of $4 \pi k^{2} d k$. To avoid counting the same state more than once only the positive octant need be counted and adding a factor of 2 from the spin degeneracy gives us a solution for the number of states $(g(k) d k)$ inside the shell as

$$
g(k) d k=2 \cdot \frac{1}{8} \cdot \rho_{k} \cdot V_{k}=\frac{V k^{2}}{\pi^{2}} d k .
$$


Differentiating once and rearranging equation 1.6 then substituting into equation 1.9 and dividing by $V$ gives the oft-quoted density of electron states in the conduction band per unit volume for a bulk material [33]

$$
g(E)^{3 D}=4 \pi\left(\frac{2 m^{*}}{h^{2}}\right)^{\frac{3}{2}}\left(E-E_{C}\right)^{\frac{1}{2}} .
$$

When considering confined materials, which have a dimension smaller than the Bohr radius in some or all of the spatial dimensions, the carriers are no longer free in some or all directions and as such the allowed k-space volume is reduced in dimensionality. For 2-D and 1-D free materials, by following the same method as above it can be shown that the DOS has a different order with regards to $E$. In the 0 -D case however, which is of interest here, the electron is confined in all three spatial dimensions and as such there is no k-space volume to be filled. The filling of each state is solely defined by the Pauli principle and a Dirac delta function, that is, for conduction band electrons [34]:

$$
g(E)^{0 D}=2 \delta\left(E-E_{C}\right)
$$

For simplicity's sake the concept of the effective density of states is used. This makes the assumption that all of the band states are located directly at the band edge, i.e they have been pinned at some $E$ and in the 0-D case gives the trivial solution for the number of states in the conduction band $\left(N_{C}\right)$ as [34]:

$$
N_{C}^{0 D}=2
$$

\subsubsection{Conduction in QD Films}

Traditional semiconductor transport is entirely based around the band model of conduction. There is a large degeneracy of available states within the conduction and valence bands and physical transport occurs as excited carriers transition between these available states due to the energy pro- 
vided by an applied or built in field [19]. Explicitly, in a classical semiconductor there is only conduction at non-zero temperatures where electrons have been thermally promoted from the valence band, above the fermi energy, and into the conduction band. Colloidally suspended QDs (CQDs) when deposited on some substrate in such a way that the solvent is removed are called CQD solids (CQDS). These CQDS are 3-D materials that contain only the QDs and the organic molecules on the exterior that determine how they interact with their environment. As these are the active materials used throughout this work it is highly instructive to discuss the mechanism of conduction in these materials. As we have just seen the degeneracy in a $0-\mathrm{D}$ material is considerably lower than in a 3-D material and thus there are only two states in the conduction band ground state for each dot. Conduction in a film of QDs relies on hopping over the large barriers that exist between each QD. The conventional band model of conduction is therefore not applicable either in a single QD or in an ensemble of separated QDs. From a historical perspective the understanding of conduction in CQDS has built on the previous work on the insulating state of granular metals and the conduction between impurity states in bulk semiconductors [35]. Qualitatively this mechanism relies upon two basic assumptions. Firstly that the electronic population of the CQDs must be stable and have states available for conduction, secondly that the hopping time between the states gives rise to a detectable conductivity [36]. There are two general regimes that fulfil these criteria, tunnelling transport and hopping transport. These are simply identified by the fact that tunnelling is temperature independent whereas hopping is not [37].

Tunnelling Tunnelling transport is the problem of an attenuating wavefunction through a forbidden barrier. This is the well understood problem of quantum tunnelling, shown in Figure 1.8 and the tunnelling rate $(\Gamma)$ has 
the well known form $[37,38]$

$$
\Gamma \approx \exp \left(-2\left(\frac{\sqrt{2 m V_{B}}}{\hbar}\right) l\right)=\exp (-\beta l)
$$

This rate is most strongly affected by $l$, therefore, if a system is conduct-

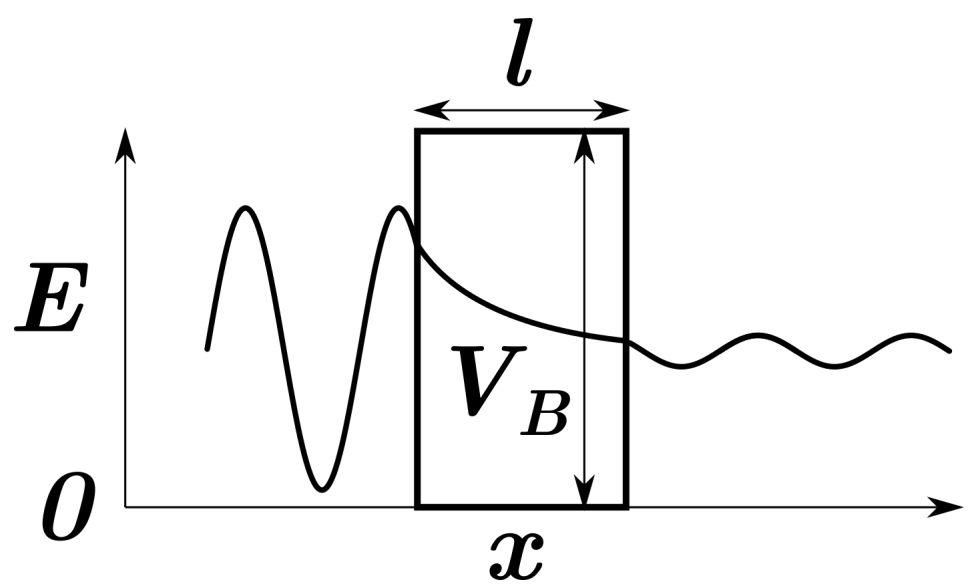

Figure 1.8: Graphical depiction of wavefunction attenuation through a forbidden barrier of height $V_{B}$ and width $l$ as seen in Equation 1.13.

ing through tunnelling transport then reducing the interdot separation is the easiest way to increase the measurable conductivity [37]. The devices studied in this work, however, all show temperature dependence of their behaviour, so are best understood through a hopping model.

Hopping As this is the central mechanism for transport in the materials that will be looked at in this thesis, both a generalised theoretical model will be given, as well as a more practical explanation of the different regimes that are commonly observed in these materials. The simplest treatment of this problem is achieved by modelling a single electron moving ballistically between two dots [36]. The energy barrier associated with the electron moving between the two is the charging energy [38]

$$
E_{a}=\frac{e^{2}}{4 \pi \epsilon r}=\frac{e^{2}}{C}
$$


where $C$ is the capacitance of the QD derived from Gauss's Law [38]. Taking the time constant $(\tau)$ as $R C$, the lowest possible limit for the resistance of the two dot system is [36]

$$
E_{a} \approx \frac{h}{\tau} \approx \frac{h}{R C} \therefore R \approx \frac{h}{e^{2}} .
$$

Extending this to a solid i.e. assuming some periodic interdot spacing $d$ the maximum possible conductivity is [36]

$$
\sigma \approx \frac{e^{2}}{h d}
$$

This very basic assumption is working on the principle that there is coherent transport in 1-D between two QDs. The more generalised version of this system is described by the Landauer formula. This describes transport in a 1-D channel between two large reservoirs of charge. If the reservoirs have different filling there is potential difference between them and a current will flow. Due to the dimensionality of the system the conductance/resistance quantises and the Landauer formula for the conductance of an electron moving from some state on the left $(i)$ to some state on the right $(j)$ is [38]

$$
G=\frac{2 e^{2}}{h} \sum_{i, j} \mathcal{T}_{i j}
$$

where $\mathcal{T}$ is the channel conductance, this has been experimentally verified [39]. As has previously been shown, 0-D materials have a degeneracy of 2 in the conduction band ground state. Therefore the overriding determinant of film conductivity is not the number of channels, as it would be for a metallic system, but the transmission $\mathcal{T}$ across the small number of available channels. Within discussion of CQDS the term hopping conduction is used to differentiate from band like conduction, implying that the electrons hop between QDs rather than existing in a band like continuum throughout the entire CQDS. Experimental measurements of trans- 
port through CQDS where the QDs remain spatially distinct have shown a temperature dependence [40] that suggests that hopping in CQDS may be a thermally activated process and the Arrhenius relationship [41] is used to describe the hopping rate in these systems. The prefactor can be taken as the time constant $(\tau)$ in equation 1.15 as this represents the absolute maximum theoretical possible rate of hopping [36], this gives

$$
\tau_{h o p}^{-1}=\tau^{-1} e^{-\frac{E a}{k T}}
$$

where $\tau_{\text {hop }}$ is the effective hopping time. Combining equations $1.15,1.17$ and 1.18 gives $\tau_{\text {hop }}$ as:

$$
\tau_{\text {hop }}^{-1}=\frac{2 E_{a}}{h} \Gamma e^{-\frac{E_{a}}{k T}}
$$

It is worth noting here that the $\Gamma$ used above is the one derived for the tunnelling case, now modified to include a temperature dependence. The Einstein relation between the mobility and diffusion of charged particles in three dimensions [19], if applied in the condition where the carrier lifetime is taken as the hopping time and the diffusion length is set as the interdot distance gives [42] [43]

$$
\mu=\frac{e d^{2}}{6 \tau_{\text {hop }} k T} .
$$

In the single channel approximation with thermally activated hopping the expression for the mobility of carriers in a CQDS is thus given by combining equations $1.13,1.19$ and 1.20 [36].

$$
\mu=\frac{e d^{2} E_{a}}{3 h k T} e^{-\beta l-\frac{E_{a}}{k T}}
$$

Experimental measurements have given the value of $\beta$ for various alkane ligands as $0.11 \mathrm{~nm}^{-1}$ [44]. More qualitatively this equation explains the importance of ligand length on determining electronic conduction. It is worth noting that there is unlikely to be only a single channel between QDs due to level degeneracy so measured mobility could be expected to 
be up to four times higher for a two-fold degenerate system. It is also essential to note here that this is an expression for the mobility of a carrier in an idealised theoretical system. The actual conductivity of a system will also be heavily influenced by, primarily, the number of carriers at any temperature.

The general form of hopping over all length scales is shown schematically in Figure 1.9 which shows an electron hopping from the $i$ th to the $j$ th QD in a low temperature system where that hop is more likely than a closer hop due to the relative activation energies of the local dots. Using the same notation as for the case above and with $n=j-i$ and $r=n d$ the conductance can be approximated as [36]

$$
G_{i \rightarrow j} \approx \exp \left(\frac{-2 r}{\xi}-\frac{\Delta E_{i j}}{k T}\right),
$$

where the solution has been formalised in such a way that $\xi$ which has the dimension of length, is called the localisation length and has the value $\xi=\frac{2 d}{\beta l}[36,45]$. At temperatures above $T \approx \frac{E_{a}}{k}$ the behaviour that is ob-

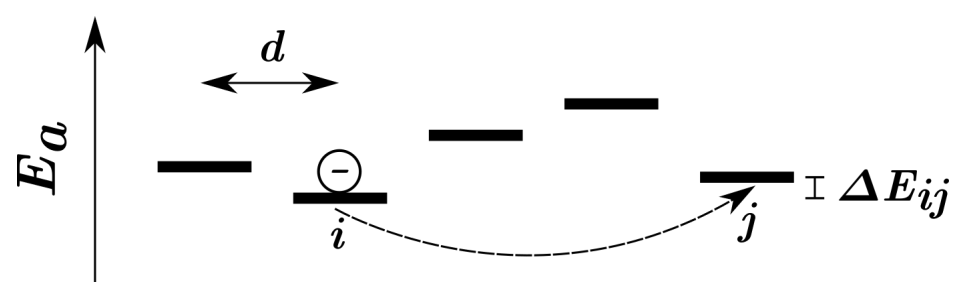

Figure 1.9: Graphical depiction of VRH in a system of spatially separated QDs with differing activation energies. Carriers can hop to the sites that are not nearest neighbour sites if the distribution of activation energies in the system creates a situation where a larger hop is actually more likely than a shorter one.

served is that the total probability (Equation1.21) leads only to hopping between nearest neighbour QDs, this is termed nearest neighbour hopping $(\mathrm{NNH})$. It has been suggested that the observed behaviour at high temperatures that is commonly described with the Arrhenius relationship is actually just the temperature domain where $\mathrm{NNH}$ is dominant [46]. As 
the temperature decreases below $T \approx \frac{E_{a}}{k}$ the mechanism in a domain where it is called variable range hopping (VRH). As an electron will look to maximise hopping probability when in an electric field, in order reach a minimum energy state. There will be some temperature below which the activation energy of the nearest QD is actually higher than the activation energy of a QD further away and it will be more likely that the electron hops to a QD that is not a nearest neighbour [45,47], the lower tunnelling probability is offest by the smaller difference in activation energy . This argument actually depends on a physically realistic system where the charging energy of each dot is different rather than an idealised system where each dot is identical. This was first formalised by Mott and showed a $\exp \left(T^{-\frac{1}{4}}\right)$ dependence (M-VRH) [48]. However a large number of systems instead showed a $\exp \left(T^{-\frac{1}{2}}\right)$ dependence termed EfrosShklovskii VRH (ES-VRH) [49]. The difference between the two theories is how the coulomb interaction affects the density of states (DOS) at the fermi level. ES-VRH takes the coulomb interaction of the carriers into account and suggests that a coulomb gap appears at the fermi level, thus the hopping behaviour is dimensionally independent and only dependent on the thermal kinetics of crossing this gap. The current understanding is that both regimes exist with transitions between them. At the lowest temperatures the CQDS show ES-VRH behaviour. Above this there is an M-VRH regime and then a further transition to $\mathrm{NNH}[36,45]$. The ES-VRH to MVRH transition occurs at the point at which $\Delta E_{i j} \approx 2 E_{a}$, i.e. below the temperature at which this occurs the coulomb gap cannot be thermally crossed. These behaviours are restricted to semiconductor QDs as they have a small and variable DOS around the fermi level. Metallic QDs, for example would not show M-VRH as the DOS at the fermi level is very large and therefore the narrowing of the band with temperature will still present a large number of spatially close states for hopping. 


\subsection{Current Detector Technology}

There are a large number of competing materials and detector designs that are, or have been used for IR detection $[3,50,51]$. Due to the focus of this thesis on the metal chalcogenide semiconductors this section will discuss Mercad Telluride (HgCdTe, MCT) alloys [52] when there is a choice of materials that serve similar functions.

\subsubsection{History}

PC devices were the first to appear in the mid 1960s [52], closely followed by the first PN photodiodes. The first scanning (i.e. suitable for imaging) devices were developed from PC technology in the 1970s and although further progress was seen until 2000 they have become largely obsolete. The first multi pixel integrated displays based on photodiodes appeared at the end of the 1970s [50]. With the increasing expertise in micro-scale device manufacture following on from the rapid decrease in transistor feature size, the first $\mathrm{P}^{+} \mathrm{N}$ and $\mathrm{N}^{+} \mathrm{P}$ photodiode designs appeared in the 1980s. These allowed a greater absorption in the depletion region, converting a greater proportion of incident power into output signal [22]. The 1980s and 1990s saw an increase in complexity of device design and operating principles with avalanche photodiodes and quantum well and layered devices becoming more common. By the middle of the 1990s, Focal Plane Arrays (FPA - multi pixel devices) were edging towards one megapixel [50,53]. Since the year 2000 the Strained Layer Superlattice has emerged as a leading contender for the future of devices in the MIR and LWIR and the operating temperature of many devices has been increasing upwards from $77 \mathrm{~K}[3,52]$. Quantum dot detectors (QDIPs) as opposed to QD in a well detectors (DWELLs) have now become more common, the current QDIP highpoint of MIR detector research being the background limited devices produced by Philippe Guyot-Sionnest et al. [21,54]. Aside from the recent QDIP devices all of the best performing devices to date 
are still highly complex and expensive epitaxial layered devices. Little has been done to rectify the major trade-offs in production cost and operation.

\subsubsection{Thermal Detectors}

Thermal detectors (bolometers) operate by means of detecting a change in the temperature of the detector material by observing a change in an electrical parameter, usually resistance $[17,51]$. The change of temperature is dependent upon the energy of the incident radiation. Noting that the transducer is wavelength indiscriminate as the material will absorb a range of wavelengths and each will heat up the detector, any device spectral sensitivity is achieved through the use of optical cut on filters and as such they are not as sensitive to the incident spectra as quantum based detectors. Bolometers are also inherently limited by background noise or by thermal response time, as both are related to the thermal conductance of the detector material, one proportionally and one inversely [4,20]. Thermal detectors can be scaled into an array suitable for imaging. These are called micro bolometers and currently offer the only workable uncooled IR imaging devices [55]. Bolometers remain difficult to produce as the detector material must be thermally isolated from the environment and the transducer must be in a local vacuum $[4,20]$.

\subsubsection{Bulk Semiconductors}

Bulk PN detectors are the most well established form of quantum IR detector. The two most common materials currently in use are MCT (MerCad Telluride) [5] and InSb [52], bulk epitaxially grown crystals of which are integrated into a circuit. The creation of array devices follows simply by dividing the bulk crystal into individual sensors using various photolithographic methods with vertical electrical connections [24]. MCT is commonly used as varying the $\mathrm{Hg} / \mathrm{Cd}$ ratio allows the bandgap to be tuned from $0 \mathrm{eV}$ where $\mathrm{HgTe}$ is a semi-metal, to $1.5 \mathrm{eV}$ where MCT is a direct 
bandgap semiconductor, thus covering the entire IR regime.

In addition to this tunability, the characteristics of MCT are a direct energy gap, high electron mobility, low dielectric constant and a relatively constant lattice constant with changing cation composition $[52,56]$. These mean that MCT lends itself well to large scale manufacturing at various detector wavelengths.

Advanced Bulk Quantum Detectors As an example of the development of bulk detectors one of the most common designs of quantum detector, the Quantum Well Detector (QWID) will be briefly discussed. The key feature of QWID designs is the use of two materials with different bandgaps [57-59]. The structure consists of a quantum well layer between two barrier layers. The conduction band ground state of the well layer is at a lower energy than the barrier conduction band.

When incident photon energy is equal to or greater than the conduction band offset energy then an electron that absorbs that photon will be in its first excited state at or above the ground state of the barrier layer material. This electron is then free inside the material, analogous to the excited electron of the EHP in a photodiode. It can then be accelerated by an electric field over the device, contributing to the photocurrent in the circuit [58]. In order for there to be sufficient electrons in the ground state of the well layer it must be accurately doped, placing the first constraint on material selection. The conduction band offset must also be tuned to the desired energy. For shorter wavelengths this is difficult as very different materials will have larger lattice mismatches [59] so theoretically suitable materials cannot be put together physically without major lattice mismatch and hence large defects. Another major constraint is that due to the polarisation selection rule, normally incident light can only induce an intraband transition if the polarisation of the light is in the growth direction [57]. The expectation for advanced quantum well detectors is that they will outperform bulk MCT due to the suppression of Auger recom- 
bination [60] and tunnelling effects [61]. The first is an expected result of the spatial electron/hole separation, whereby the holes localise in the lower bandgap material and the electrons in the higher bandgap material. The reduction in tunnelling is expected to arise due to the higher effective mass of the carriers in the materials used when compared to MCT, leading to a lower tunnelling probability out of the conduction band ground state, which is a source of dark current.

\subsubsection{Detector Characteristics}

There are a small number of commonly used parameters that allow comparison between different detectors. The most commonly used are below $[18,22,62,63]$ :

1. External Quantum Efficiency. The efficiency with which the device collects incident photons and converts them into current at the output electrodes [20,62].

$$
\eta_{e}=\frac{\text { number of collected electrons }}{\text { number of incident photons }}=\frac{\frac{I_{p h}}{e}}{\frac{P_{O}}{h v}} .
$$

2. Responsivity. The photocurrent generated per incident optical power at a given wavelength of incident light. This provides a spectral response so the peak value is usually quoted. This is a measure of a detector's ability to convert incident light into electrical signal $[20,62]$ :

$$
R=\frac{I_{p h}}{P_{O}}=\frac{\eta_{e} e}{h v} .
$$

3. Noise Equivalent Power (NEP). This is the optical signal power required to generate a photocurrent that is equal to the total noise current $I_{n}$ at a given wavelength and $1 \mathrm{~Hz}$ input bandwidth $(B)$. Some- 
times this is seen as the noise equivalent difference in temperature (NEDT), which is a measure of the temperature difference in signal required to overcome the detector noise. NEP is usually quoted in watts or watts per square root of frequency. If the device is limited by thermal noise the NEP can also be called the sensitivity, as it defines how sensitive the detector is to optical signals [20,62]:

$$
N E P=\frac{V_{n}}{R}
$$

4. Specific Detectivity. The reciprocal of the NEP is defined at the Detectivity $(D)$ of a device. To normalise the quantity across device structures the specific detectivity $\left(D^{*}\right)$ is commonly used. It is a measure of the sensitivity of a device to total noise, accounting for the electrical bandwidth of the input signal and the area of the active device area. The unit is called a Jones $\left(\mathrm{cm} H z^{\frac{1}{2}} W^{-1}\right)$ [20,62]:

$$
D^{*}=\frac{\sqrt{A B}}{N E P} .
$$

5. Background Limited Infrared Photodetection (BLIP) [63]. This is a term used to describe operation of a photodetector when the total density of photogenerated carriers is greater than the density of thermal carriers. The limiting noise of the detector is thus governed by the background flux rather than other inherent detector noise. It is important because it shows that the detector is performance limited by the nature of EM radiation rather than by any imperfections that exist in its design. It can be calculated by using the NEP for the specific BLIP case and the background flux:

$$
N E P_{B L I P}=h v \sqrt{\frac{2 \Phi_{B} B}{\eta_{e}}} .
$$

It can also be quantified by an evaluation of the following inequality 
[64]. If satisfied the detector is operating under the BLIP condition, where $\tau$ is the carrier lifetime:

$$
\frac{\eta_{e} \Phi_{B} \tau}{\text { sample thickness }}>n_{i}
$$

\subsubsection{Detector Noise}

Any device that returns an electrical signal will have various types of noise that degrade or mask the output signal. Noise being defined as an output electrical signal that is not coherent with the input signal. It is worth noting that the dark current is not noise as it is a quantifiable signal that is predictable in the future, whereas true noise is not predictable at any future point. The most relevant are listed below:

1. Photon Noise. There is an inherent noise due to any source of photons, whether the intended source or the background. This is a quantum noise due to the discrete nature of EM radiation and the Poisson distribution of independent random events. The noise can be calculated as the square root of the number of events. The finite value of the noise then increases as the number of events increases, however the relative ratio, i.e. the signal to noise ratio, will be much lower for a small number of events. The obvious fallout from photon noise is that testing should be done with the greatest intensity that still accurately represents the desired test parameters. This noise will always result in an irreducible minimum noise in the output signal $[17,22,62]$.

2. Johnson/Thermal Noise. Thermal motion of charge carriers within an electrical circuit above absolute zero will result in a random movement of charge throughout any resistive device. The noise caused by this thermal motion is the Johnson noise of the detector. It is a white noise proportional to the absolute temperature of the 
circuit which can be well quantified as [65]:

$$
V_{\text {Johnson }}^{2}=4 k T R B
$$

where $T$ is the temperature of the device, $R$ is the measured resistance and $B$ is the bandwidth.

3. Shot Noise [22]. As with photon noise there is a noise current that arises from the random and independent movement of charge carriers across any depletion layer, even if thermal motion is minimised. This is commonly described as a shot noise current. It is used to calculate the quantum noise limit of PN junction based detector systems.

4. 1/f Noise. This is a term that refers to noise with a power spectrum that decreases inversely with frequency. Although not well understood it is thought to relate to the structure of the material itself, in particular trap states and grain boundaries. Recent work has well characterised the behaviour of this noise for NC solids and outlined methods to take to reduce it [66].

\subsection{Quantum Dots}

\subsubsection{Theoretical}

QDs and NCs are clusters of atoms that have sizes in all three spatial dimensions that are close to or less than the Bohr radius of the bulk material so excitons in the NC are therefore completely confined and cannot move. It is for this reason that they are called $0-\mathrm{D}$ materials $[67,68]$. The exciton is a quasi-particle that exists in a semiconductor and consists of the energetically promoted electron and the newly vacated hole; it can be considered as a hydrogen-like atom moving inside the material. In general the exciton Bohr radius in a semiconductor is, due to the lighter effective 
mass and larger permittivity, considerably larger than the hydrogen Bohr radius, for example HgTe has a Bohr radius of $40 \mathrm{~nm}$ [56]. Therefore confinement effects will be seen for HgTe NCs with dimensions less than 40 $\mathrm{nm}$. The most striking confinement effect, and the one that has the most application to this thesis, is the variation of bandgap $\left(E_{g}\right)$ with NC size, a brief theoretical explanation of which will be given below. Arguably the most intuitive qualitative description follows from the Uncertainty Principle [69]. The act of constructing a NC localises the particle so that the uncertainty in the position can be modelled as the radius of the $\mathrm{NC}$, thus the uncertainty in the momentum varies inversely with the radius. It can therefore be seen that the energy of a particle constrained in the dot will have an energy that varies inversely with the square of the radius for a given particle effective mass [67].

Semiconductor Nanocrystals For semiconductor NCs the confinement effect is seen optically as an increase in bandgap energy with decreasing NC size. The first observation of this size dependent optical behaviour was in colloidal CdS QDs in 1983 [70] although it had been observed prior to this in molecular beam deposited 1-D GaAs layers [71], although these devices did not have a well controlled thickness so were not true 0-D materials [72]. Modelling the NC as bulk semiconductor lattice, i.e. a system that is solved with a periodic Bloch wavefunction [73] that is further modulated by a spatial envelope function that represents the QD volume, shown in equation 1.30 , allows this change in bandgap to be modelled $[73,74]$.

$$
\Psi_{N C}(x)=\psi_{B l o c h}(x) \Phi_{e n v}(x)
$$

The solution to which would be another solution to a 'particle in a 3-D box' problem, however modelling the $\mathrm{NC}$ as a sphere, i.e. a spherical envelope function that is a product of a spherical harmonic function and a Bessel function (equation 1.31), allows for a solution that mirrors the solution for 
the energy levels of the hydrogen atom.

$$
\Phi_{e n v}(\theta, \phi, r)=Y_{l}^{m}(\theta, \phi) R_{\text {Bessel }}(r)
$$

The potential that is experienced by the electron in this model is shown in equation 1.32. $D$ is the the diameter of the $\mathrm{NC}$ and the potential inside the $\mathrm{NC}$ is modelled as a constant finite value, $V_{0}$.

$$
V(r)=-V_{0} \text { for }|r|<\frac{D}{2}, V(r)=0 \text { for }|r|>D / 2 .
$$

Solving the Schrödinger equation for the envelope function gives solutions for the energy levels of a confined electron.

$$
E_{n, l}^{c o n f}=\frac{2 \hbar^{2} \chi_{n, l}^{2}}{m^{*} D^{2}}
$$

These energies are similar to the energy levels of an electron in a hydrogen atom however due to the differing potential there is no longer a restriction of $l$ with $n$, e.g. NCs have $1 \mathrm{P}(n=1, l=1)$ and $1 \mathrm{D}(n=1, l=2)$ energy levels. If the full wavefunction for the NC is solved the bandgap that appears is modulated in energy by the confinement energy and becomes [74].

$$
E_{g}^{N C}=E_{g}^{b u l k}+\frac{2 \hbar^{2} \chi_{n, l}^{2}}{m_{e}^{*} D^{2}}+\frac{2 \hbar^{2} \chi_{n, l}^{2}}{m_{h}^{*} D^{2}}
$$

The above is a restatement of the Brus equation [75]. It can therefore be seen that the spatial confinement of the exciton will cause a shift in the bandgap energy, which is shown in Figure 1.10. In terms of terminology, if the QD is smaller than the exciton Bohr radius in the material the QD is in the strong confinement regime. If the QD is larger than the Bohr radius the system exists in a weak confinement regime [76]. Due to the large Bohr radii of the materials used in this work, and the small QD size, this thesis is concerned only with the strong confinement regime. 


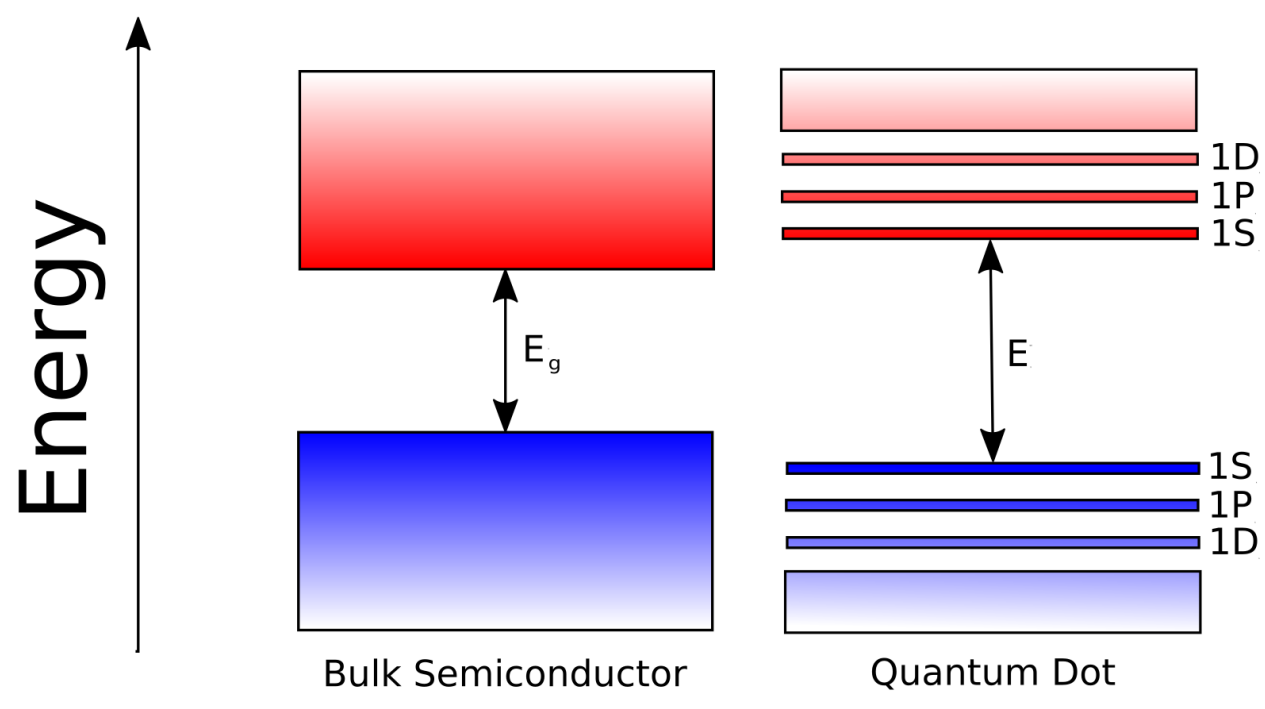

Figure 1.10: Representative cartoon showing the energy levels due to confinement relative to those found in the bulk material for a semiconductor NC.

\subsubsection{Colloidal QD Synthesis}

Colloidal preparations currently offer the best methods to create electronic grade QDs that can be used as the sensitising and charge carrier medium in devices [32]. This method is characterised by the use of ligands to separate the QDs and allow them to disperse in solution [77]. The distinction between colloid and solution here is not overly important. Technically the solutions are colloids as the suspended particles are larger than single molecules, however colloids are commonly "solution" processed and the two terms are interchangeable. Chemically, the important point is that these QD/solvent mixtures should not settle out due to sedimentation. This is favourable as the QDs can be further processed directly from solution. This opens up the use of thin film processing techniques such as spin coating, printing and stamping as a means of depositing QDs onto a wide variety of substrates. These methods are generally much lower in cost and easier than physical vapour deposition methods, which facilitates 
faster prototyping and testing of a wide variety of device structures without large initial investment. Essentially, CQDs offer solution-depositable semiconductors. The secondary benefit of the colloidal method is that the surface of the QD is passivated by organic ligands which offer another opportunity to alter the macro properties of NC assemblies [78].

History The first CQDs were synthesised via arrested precipitation [79]. This is a method that relies upon the very low solubility of binary semiconductors in aqueous solutions. If two metal salt precursors are both super saturated in the solution there exists an energetically favourable pathway to precipitate the binary semiconductor. This method although successful led to very little size control as the primary method was to alter the super saturation point of the colloid via a change in $\mathrm{pH}$, therefore limiting further nucleation events and increasing QD size via addition from ions still in solution. The unintended side effect of this method being the broadening of size distribution as some QDs were dissolved and others grew via Ostwald ripening $[72,79]$. The quest for greater size control led to templated growth methods, the most numerous and successful being the use of biological micelles to provide a physical boundary for possible growth. This method was effective at providing a finer degree of size control [80] however the line broadening of emission and absorption were far larger than the expected values [81]. This is now known to be due to a large distribution of un-passivated surface states that serve as trapping centres. As the surface of a QD is a large fraction of the whole, the broad spectrum of surface trap states completely obscured the sharp homogeneous absorption that was expected from a quantum confined system $[72,78]$. It was found that the addition of cations to QD solutions served to enhance photoluminescence (PL) measurements, whereas anions quenched it [82]. It is now understood that hole trap states localised at surface anions are passivated by the addition of cations and this is responsible for the dramatically improved absorption and PL line widths [83]. The now ubiquitous use of 
organic ligands as surface passivators underpins the generic method of organometallic colloidal NC synthesis and this method, as used in this thesis, was first pioneered in the preparation of $\mathrm{CdX}(X=S, \mathrm{Se}, \mathrm{Te})$ [84] and later PbSe QDs [85]. The growth of QDs in these colloidal syntheses is based upon the LaMer model [86]. This states that monodisperse nanoparticles in a colloidal suspension require a distinct nucleation event that is followed by a period of growth when new particles are no longer being created. It is found that this nucleation event is given by a threshold concentration of precursors that while exceeded will nucleate new particles, see Figure 1.11. The most common method for achieving this is via an injection of one precursor, most commonly a tertiary phosphine stabilised chalcogenide, into a heated solution of the other (for a binary NC). The heated solution will contain an ionic precursor for the cation, likely in solution with a primary amine as these are now known to be the most effective co-ordinating solvent [87]. The size of the NC is governed by the length of time spent in the growth solution, at a certain temperature. This allows the time and temperature to be tuned to achieve the desired dispersion and NC dimensions.

Capping Ligands The growth endpoint is determined by the introduction of long chain aliphatic molecules to the growth solution. The head group of these ligands covalently bond to each NC and separate individual NCs from each other. Growth is halted as the NCs no longer interact with each other, or any remaining precursor in the growth solution. The ligands chemically separate the NC from its environment and thus are a major factor to consider when considering the structure or behaviour of any NC assembly $[23,78,88]$. The general model for ligand bonding is that a headgroup will covalently bond to the NC surface and the hydrocarbon tail will point away from the surface. In this way the ligands can be thought of as a self assembled monolayer (SAM) that covers the surface of each NC, see Figure 1.12. The goal is an NC dispersion that is fully stable 


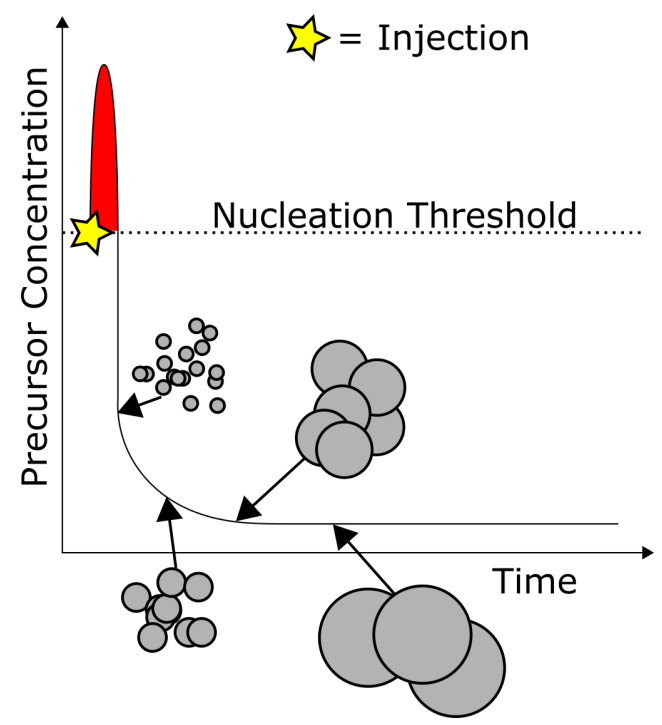

Figure 1.11: Graphical depiction of the Lamer NC growth method. The injection of one of the precursors above the nucleation threshold concentration causes QDs to nucleate for the period shown in red. Once below the nucleation threshold no new QDs are formed and the number of $Q D$ s decreases whilst the average $Q D$ size increases.

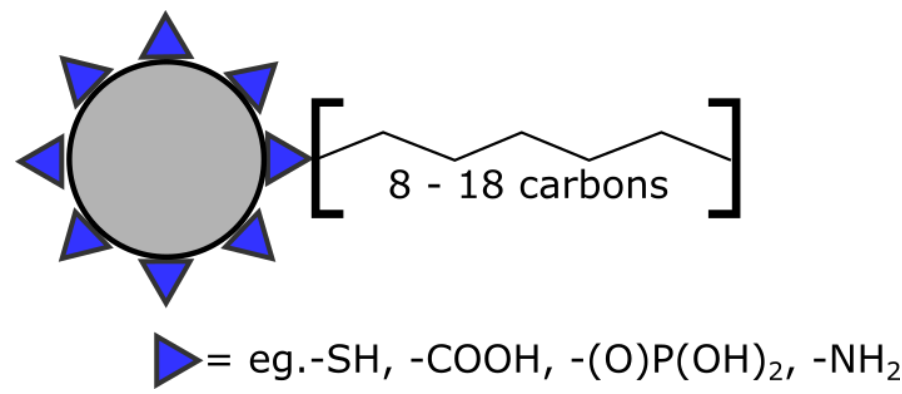

Figure 1.12: Aliphatic ligands covalently bonding to NC surface the ligand most commonly used in this work for stabilised QDs is the thiol (-SH) group.

in solution, as this colloidal NC "ink" is what gives flexibility to NC device manufacture. Colloidal stability is governed by the interaction of the visible NC surface bound hydrocarbon chains, with the chosen solvent. Solvents that have a negative free energy of mixing with the hydrocarbon chains will be good solvents, as they create a repellent force between individual NCs, preventing aggregation [89]. In the general metal chalco- 
genide case outlined above these good solvents will be non-polar. Conversely polar solvents will cause a positive free energy of mixing, forcing NCs together and causing aggregation. These bad solvents are referred to as anti-solvents and their use will be seen in the experimental Chapters. As well as determining the solubility of the NCs, ligands can also be swapped and tuned via surface chemistry to affect macro parameters of NC assemblies such as conductivity [44] and inter-NC separation [90]. Ligand engineering affects the exciton lifetimes in the QD as ligand choice can passivate surface states, minimising inter bandgap states that allow absorption pathways that result in reduced responsivity, i.e. a band edge that is not sharply defined (analogous to Non Radiative Recombination (NRR) pathways in light emitting systems), shown in Figure 1.13 [23]. The larger problem of why bandgaps do not show square wave like absorption behaviour is due to the Urbach Rule [91]. In the case of NC absorption edges the size dispersion of the sample adds another factor as differently sized NCs will actually have different bandgaps. The band edge will be ill-defined even before the conventional Urbach edge effects are applied. When looking at absorption measurements the exponential decay of absorption at energies below the bandgap is called the Urbach tail. In NC samples it is a good indicator of sample size dispersity, with small Urbach tails indicating a small size dispersion. Since being introduced by Kovalenko et al. in 2009, the use of inorganic ligands has also become possible. Short inorganic ligands allow very small inter QD spacing, resulting in very high carrier mobilities [92,93].

HgTe Nanocrystals MCT is an excellent material for bulk IR detectors due to its bandgap tunability, and this same tuneability can be achieved with HgTe NCs [5,94]. The bandgap of bulk HgTe is $-0.141 \mathrm{eV}$ at $300 \mathrm{~K}$ [95] so it can be expected that the NC bandgap can be engineered to be small enough to absorb MIR radiation. The concept of a negative bandgap is related to the energy levels of different molecular orbitals (MO). In the 


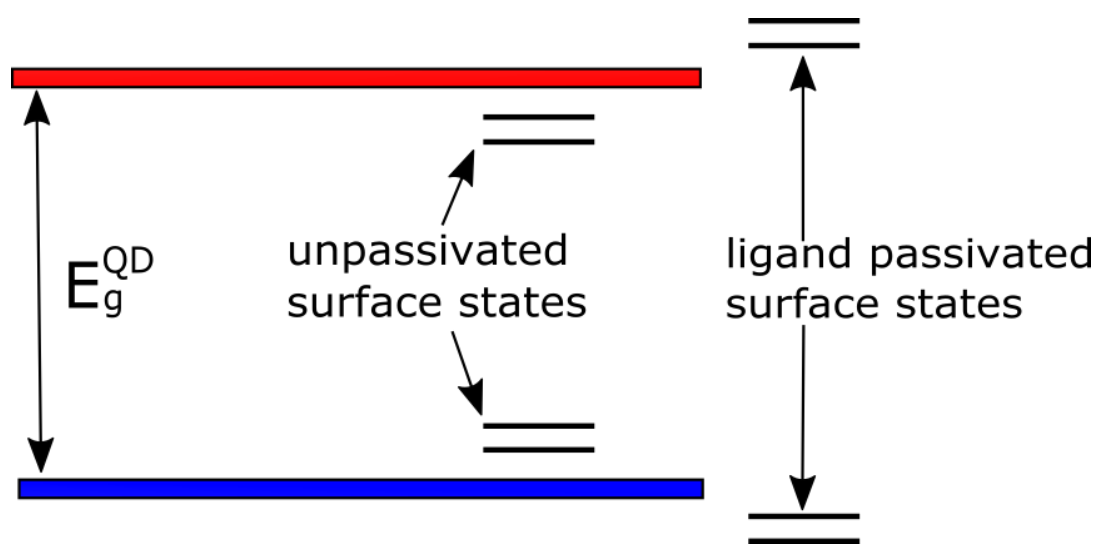

Figure 1.13: Cartoon showing the effect of successful ligand passivation on the energy levels of surface states. If the ligand passivated states are not in the bandgap then a successfully passivated $Q D$ has a number of non radiative recombination pathways removed.

case of HgTe, at the wavevector where the direct bandgap occurs there is an inversion of energy levels whereby a lower energy band occupies a higher energy than another band that is conventionally above it [96]. It can be envisioned as the top of the filled valence band being $0.141 \mathrm{eV}$ above the bottom of the unfilled conduction band. Of particular interest for this proposal are dots sized between 6 and $10 \mathrm{~nm}$ as these correspond to bandgaps that bridge the MIR. The metal-organic NC route that is the foundation for the current MIR QDIP sensitiser layers was first proposed by Green et al. in 2003 [97], before being improved upon by Keuleyan et al. $[94,98]$ and Lhuillier et al. $[99,100]$.

\subsection{Quantum Dot Infrared Photo Detectors (QDIPS)}

Quantum dot infrared photodetectors use QDs as the light sensitive medium, as opposed to layers of bulk semiconductor. The first MIR devices of this kind were well suited to a similar construction method as quantum well detectors, as one of the initial growth methods for the 
first QDs was epitaxial. This method was first achieved in 1993 [101]. However, due to the proliferation of aqueous [102,103], and organically passivated colloidal $[32,97]$, methods of HgTe QD synthesis, there has been a marked increase in the variety of QDIP device structure [6, 23,77].

\subsubsection{Quantum Dot use in Detectors}

Semiconductor NCs are an attractive material to use in detectors because they can be thought of as a homogeneous film that acts as both the absorber and transport layer $[23,104]$. This allows for structurally simple device designs. Conceptually the desired mode of operation can be chosen via the selection of contact materials, generally either an ohmic contact, schottky barrier or ETL/HTL layer, and mode of operation, for example the device bias and gating. Coupling simple device design with solution processable absorber layers makes QDIPs a very attractive proposition over complex MBE deposited QWIPs, both in terms of simplicity and cost. Further adding to the practical benefits there are a number of theoretical reasons that QDIPs should easily outperform QWIPs and possibly bulk detectors at higher temperatures [64], as outlined below:

1. High Temperature Operation. The operation of photonic detectors without the need for cooling would represent the next step forward in IR detector technology and the unique conduction mechanism of QD solids allows one potential solution to this problem. Due to the low density of states in 0-D materials it is highly improbable that even at $300 \mathrm{~K}$ there will be a carrier thermally excited into the conduction band of an individual dot. Operation at room temperature then requires only that excited carriers can be conducted out of the dot before they recombine and that the population of photogenerated carriers exceeds that of thermal carriers, which due to the low density of states applying individually to every dot in the ensemble is predicted to result in a lower dark current than bulk materials of 
the same bandgap $[5,36]$.

2. Dark Current. As previously stated the dark current in a detector is the key determinant of the detector generated noise current. The dark current is the current flowing in the device when there is no external signal current. For any given temperature, applied field and mobility the dark current of a detector is dependent upon the carrier density in the conduction band due to thermal generation (equation 1.3). It is predicted that the reduced density of states [67] will lead to a dark current that is less than bulk HgCdTe for monodisperse QD assemblies [64].

3. Bandgap Tuning. The other avenue for reduction in dark current is via the use of bandgap tuning. Thermal generation is dependent upon bandgap, so for a given temperature the thermal generation will be reduced as the bandgap increases, another reason why MIR detectors are preferable to LWIR detectors in terms of detector noise. Therefore the bandgap of a detector should ideally be tuned to be at the lowest required temperature to be observed. Any lower than this and the detector dark current is needlessly higher than it has to be. The bandgap tuning of QDs can itself be a method to reduce detector dark current

4. Gain through Charge Trapping. If trap states, in place due to either surface effects, impurities or doping, are energetically situated such that one of the charge carriers types from each generated EHP is likely to be physically trapped the system can see PC gain. For the duration that the carrier is trapped, its partner can be multiply cycled throughout the electrical circuit and therefore be registered as more than one charge for that single photon $[22,23]$. Figure 1.14 shows this where the electron is trapped and the hole can recirculate. This gain affects all generated EHPs, both photogenerated and thermally generated so the dark current will see a corresponding gain effect, 
making this more useful in systems with an already minimised dark current.

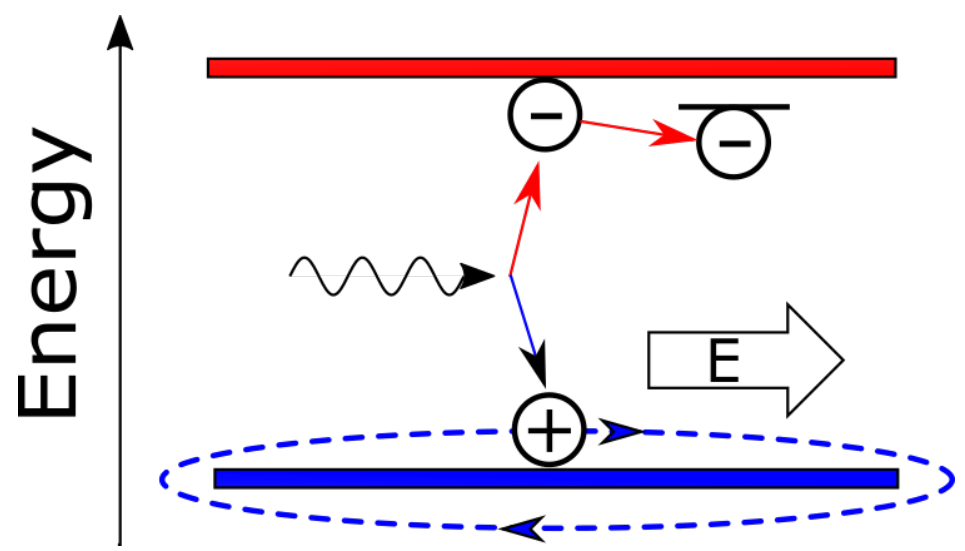

Figure 1.14: Cartoon showing the method of $P C$ gain in a conductive solid that is trapping an electron for a time sufficient for a hole to cycle through the circuit ( $E$ is electric field).

5. Phonon Bottleneck. This idea suggests that if the discrete energy states in the dot are separated such that they are larger than the phonon energy in the material, then there is one less non-radiative recombination pathway. This is termed the phonon bottleneck and if it exists directly leads to longer carrier lifetime [105].

6. Suppression of Auger recombination. Due to the reduced free carrier density in QDs compared to the bulk it is expected that Auger decay will be suppressed [54]. The far lower carrier density heavily reduces the chance that there is a third carrier to facilitate this decay pathway. This would cut off a non-radiative decay pathway and increase the exciton lifetime, although at the expense of lower absolute currents.

Possible downsides of QD use in devices include doping that is insufficient to fill the valence band states and/or insufficient dot density within the absorption length both leading to incident photons being wasted [106]. 
Auger decay and recombination rates could also be increased due to the physical localisation of the electron and hole following generation [32].

The key factors that will underpin the success of delivering on the theoretical potential are:

1. The preparation of highly monodisperse colloids. Any band smearing due to size dispersion will reduce measured conduction as the increased energy difference between adjacent sites will reduce hopping rates. Additionally an ill defined bandgap renders bandgap tuning for spectral sensitivity less effective [5].

2. Practical methods that create highly conductive QD solids while still retaining physical separation of each QD.

3. Ligand exchange procedures that do not leave a partially passivated surface. Partial or easily degraded passivation causes a large number of trap states that inhibit photoresponse rise and fall times. This can be extended to the issue of oxidation of surface cation sites that can create a high carrier density that gives conductive but photo-inactive solids.

Aside from the theoretical reasons outlined above that may allow higher responsivity from QDIP devices there are also a number of practical reasons why using colloidal QDs in QDIP devices will address the large cost and large size aspects of current detectors. They are covered elsewhere in the text but to highlight the key areas:

1. Colloidal QDs can be synthesised and processed in a normal wet chemistry lab. The process does not require a cleanroom. This can drastically reduce production costs.

2. The QDs can be applied to the device structure using solution processing techniques. Well designed solution based processes are highly scalable. 
3. Due to the quantum confinement effect QDs of different size offer different spectral sensitivity but can be produced and processed with similar techniques.

4. The very low volume of QD thin films and the flexbility of device manufacture means that cooling, if required, will be easy to achieve.

\subsubsection{Current QDIP Devices}

The major milestones in the field of QD MIR detection are summarised below, including tangential work that provided key insight into devices, deposition or surface engineering. The basic planar device structure that has proved highly effective as a simple test bed for CQD films is the inter digitated electrode (IDE) array, simply put this is a collection of transistor trenches that allows low conductivity materials to give measurable results due to a very large effective trench width, essentially a very big transistor but with an easily coverable surface area. This was popularised in 2006 by Konstantatos et al. [107].

The first reported use of HgTe QDs was by Böberl et al. in 2007 [108], an inkjet printer was used to print the dots onto Ti/Au IDEs. The devices functioned at room temperature and had a maximum responsivity of $65 \mathrm{~mA} \mathrm{~W}^{-1}$ for $10 \mathrm{~V}$ bias however this was for radiation with a wavelength of $1.4 \mu \mathrm{m}$ so SWIR rather than MIR [108]. Due to the very low noise current the reported specific detectivity was $3 \times 10^{10}$ Jones, a very impressive value considering the QDs were still surrounded by insulating ligands.

In 2009 Kovalenko et al. showed that metal chalcogenide complexes could easily replace long chain hydrocarbons as the capping ligands for NCs and nanowires (NWs). Doing so led to large improvements in electrical connectivity in NC solids but did not affect the quantum confined behaviour of the materials [92]. This was followed in 2010 by the definitive work regarding the replacement of long chain hydrocarbon ligands with 
shorter chain organics for semiconductor QDs [44]. Both of these works paved the way for the rapid development of high performance CQD devices that followed.

The true utility of HgTe QDs was explored in 2011 and showed bandgap tuning from 1.3 to $5 \mu \mathrm{m}$ with clear excitonic peaks. The synthesis was a two-step hot injection method using organometallic precursors and allowed size tuning through the temperature of the reaction. The reported QDs were highly monodisperse and even showed photoluminescence in the MIR. The QDs were tetrahedral in shape due to the differing growth rates on each facet of the zinc-blende crystal at the low temperatures used [98].

The first MIR CQD photodetector was fabricated using drop cast solutions of HgTe QDs. The devices showed room temperature detection up to $5 \mu \mathrm{m}$. At room temperature the reported specific detectivity was $10 \times 10^{7}$ Jones in the MIR, with a responsivity of $20 \mathrm{~mA} \mathrm{~W}^{-1}$ [43]. Achieving room temperature detection of $5 \mu \mathrm{m}$ radiation with a planar device structure was the major milestone in demonstrating the potential of CQD based devices. The reported metrics are all the more impressive considering that the QDs were shown to be aggregated even in solution in TEM images.

Using the same device structure and deposition method in 2013, the next step was the replacement of the organic ligands with $\mathrm{As}_{2} \mathrm{~S}_{3}$. This resulted in a greatly enhanced mobility that is 100 times higher in the $\mathrm{As}_{2} \mathrm{~S}_{3}$ treated films when compared to the best organic ligand, ethanedithiol (EDT). The method used involved a fully air free device fabrication, as when fabricated in air the devices had a lower responsivity than both the previously reported aggregated devices and the air free devices. This was thought to be due to the oxidation of unpassivated surface regions that oxidise and then act as acceptor levels, causing an increased p-type behaviour that negatively affects performance. The devices function at room temperature but the top reported metrics are given for operation at $230 \mathrm{~K}$. Responsivity is $100 \mathrm{~mA} \mathrm{~W}^{-1}$ and specific detectivity is $3.5 \times 10^{10}$ Jones at a 
wavelength of $3.5 \mu \mathrm{m}$ [99].

In 2015 HgTe QDs were used in a sandwich style device and demonstrated the first reported background limited MIR photodetection, i.e. the device performance was constrained more by the inherent background noise than by the process of detection. Due to the sandwich nature the device showed photodiodic behaviour, generating photocurrent with zero bias. The device structure was a $\mathrm{NiCr}$ ultrathin back electrode on a $\mathrm{CaF}_{2}$ substrate. This was dip coated to get the QD layer, then $\mathrm{Ag}_{2} \mathrm{Te}$ QDs are used as a back layer before the back electrode is added with silver paint. Operation at $5 \mu \mathrm{m}$ occurs between 90 and $140 \mathrm{~K}$. The best zero bias operation is at $90 \mathrm{~K}$ with a responsivity of $86 \mathrm{~mA} \mathrm{~W}^{-1}$ and a specific detectivity of $4 \times 10^{10}$ Jones [54].

Currently the field can be broken down into four areas. The first is the pursuit of MIR performance that exceeds all known bulk material devices. In 2017 a phototransistor was demonstrated that reported a responsivity of $10^{5} \mathrm{~A} \mathrm{~W}^{-1}$ at $2 \mu \mathrm{m}$. This leap forward was achieved by using the HgTe QDs as a sensitising top gate layer on a $\mathrm{MoS}_{2}$ transistor. When charges are generated in the QDs the hole remains trapped but the electron is able to enter the gate material, and due to the high mobility of $\mathrm{MoS}_{2}$ the electron can cycle multiply though the system causing a very high PC gain. These devices are fabricated through laser and e-beam lithography on randomly exfoliated $\mathrm{MoS}_{2}$ [109] and it is unclear whether they can be reliably fabricated. Although these devices perform exceptionally they are not industrially feasible until 2-D materials can be fabricated and contacted more predictably and as such remove one of the key benefits of QD technology.

The second avenue of development is using QDs as the active layer in a transistor structure to increase the performance of air processed planar structures. Chen et al. demonstrated a silicon/silicon dioxide bottom gate transistor with spraycoated aqueous HgTe QDs as the active layer. With $12 \mathrm{~V}$ gate bias these devices demonstrate $0.3 \mathrm{~A} \mathrm{~W}^{-1}$ responsivity at $2.2 \mu \mathrm{m}$, and specific detectivity of $5 \times 10^{10}$ Jones at $2 \mathrm{kHz}$. This device shows that 


\begin{tabular}{|c|c|c|c|c|c|c|}
\hline Title & Date & $\begin{array}{l}\text { Fabrication } \\
\text { Method }\end{array}$ & $\begin{array}{l}\text { Highest } \\
\text { Operating } \\
\text { Temp }\end{array}$ & $\begin{array}{l}\text { Wavelength } \\
\text { Range }(\mu \mathrm{m})\end{array}$ & $\begin{array}{l}\text { Responsivity } \\
\text { in MIR }\end{array}$ & $\begin{array}{l}\text { Spec De- } \\
\text { tectivity } \\
\text { (Jones) }\end{array}$ \\
\hline $\begin{array}{l}\text { Mid-infrared HgTe Colloidal } \\
\text { Quantum Dot Photodetec- } \\
\text { tors [43] }\end{array}$ & 2011 & Drop cast & $\begin{array}{l}\text { Room } \\
\text { temper- } \\
\text { ature } \\
\text { (RT) }\end{array}$ & 1 to 3 and 1 to 5 & $\begin{array}{lr}3 \quad \mu \mathrm{m}: & 0.15 \\
\mathrm{~A} \mathrm{~W}^{-1} \quad 5 \quad \mu \mathrm{m}: \\
0.25 \mathrm{~A} \mathrm{~W}^{-1} @ 10 \\
\mathrm{~V}\end{array}$ & $<10^{8}$ \\
\hline 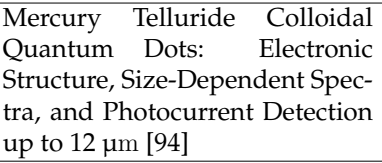 & 2014 & Drop cast & $80 \mathrm{~K}$ & MIR to 12 & $\begin{array}{ll}1.8 & \mathrm{~mA} \mathrm{~W}^{-1} \\
@ 10 \mathrm{~V} & \end{array}$ & $10^{9}$ \\
\hline $\begin{array}{l}\text { Mid-Infrared } \mathrm{HgTe} / \mathrm{As}_{2} \mathrm{~S}_{3} \text { Field } \\
\text { Effect Transistors and Photo De- } \\
\text { tectors [99] }\end{array}$ & 2012 & Drop cast & RT & 1.7 to 3 & $\begin{array}{l}0.22 \mathrm{~A} \mathrm{~W}^{-1} @ 7 \\
\mathrm{~V}\end{array}$ & $10^{9}$ \\
\hline $\begin{array}{l}\text { Background Limited Mid- } \\
\text { Infrared Photo Detection with } \\
\text { Photovoltaic HgTe CQDs [54] }\end{array}$ & 2015 & $\begin{array}{l}\text { Sandwich } \\
\text { structure, } \\
\mathrm{Ag}_{2} \mathrm{Te} \\
\mathrm{NC} \text { top } \\
\text { electrode }\end{array}$ & $140 \mathrm{~K}$ & 3 to 5.25 & $86 \mathrm{~mA} \mathrm{~W}^{-1}$ & $10^{10}$ \\
\hline $\begin{array}{l}\text { Mercury Telluride Quantum } \\
\text { Dot Based Phototransistor En- } \\
\text { abling High-Sensitivity Room- } \\
\text { Temperature Photo detection at } \\
2000 \mathrm{~nm} \text { [110] }\end{array}$ & 2017 & $\begin{array}{l}\text { Spray } \\
\text { coat, } \\
\text { aqueous } \\
\text { NCs }\end{array}$ & RT & Vis to 2.4 & $\begin{array}{l}0.3 \mathrm{~A} \mathrm{~W}^{-1} @ 12 \\
\mathrm{~V}\end{array}$ & $10^{10}$ \\
\hline $\begin{array}{l}\mathrm{MoS}_{2}-\mathrm{HgTe} \text { Quantum Dot } \\
\text { Hybrid Photodetectors beyond } \\
2 \mu \mathrm{m} \text { [109] }\end{array}$ & 2017 & $\begin{array}{l}\mathrm{MoS}_{2} \text { tran- } \\
\text { sistor then } \\
\text { spin coat- } \\
\text { ing }\end{array}$ & $300 \mathrm{~K}$ & Vis to 2.1 & $\begin{array}{l}10^{5} \mathrm{~A} \mathrm{~W}^{-1} @ 15 \\
\mathrm{~V}\end{array}$ & $10^{12}$ \\
\hline $\begin{array}{l}\text { Scalable Fabrication of Infrared } \\
\text { Detectors with Multispectral } \\
\text { Photoresponse Based on Pat- } \\
\text { terned Colloidal Quantum Dot } \\
\text { Films [111] }\end{array}$ & 2016 & $\begin{array}{l}\text { PDMS } \\
\text { stamp }\end{array}$ & RT & 2 to 7 & $\begin{array}{l}0.08 \mathrm{~A} \mathrm{~W}^{-1} @ 9 \\
\mathrm{~V}\end{array}$ & $10^{8}$ \\
\hline
\end{tabular}

Table 1.1: Table showing the best performing devices in the literature.

air processing is not necessarily an impediment to fast operation. Running this device in accumulation mode is thought to fill the hole traps near to the gate, providing a trap free region that offers unimpeded charge separation and fast device operation [110].

The third direction that CQD devices are taking is the development of multispectral detectors, using the easy bandgap tunability of QDs to fabricate detectors that offer spectral selectivity within IR spectral bands. The best demonstration of this to date was by Tang et al. who demonstrated devices that showed spectral selectivity down to less than $1 \mu \mathrm{m}$ between 2 and $9 \mu \mathrm{m}$. They also demonstrated small feature patterning with the use of PDMS transfer stamping [111]. 
Finally there is the integration of CQDs with current technology, best illustrated by the demonstration of a functioning FPA by Ciani et al [21]. This uses a spin coated HgTe film on a custom engineered read out integrated circuit (ROIC) with $30 \mu \mathrm{m}$ pitch pixels. At $95 \mathrm{~K}$ this device was able to image scenes in daylight, with an NEDT of $2.32 \mathrm{~K}$. This is another step forward and for the first time demonstrates that NC thin films can integrate with current technology to produce available commercial IR devices.

The other promising QD material within the MIR is HgSe, whereas HgTe works with interband promotion $\mathrm{HgSe}$ has shows both interband and intraband absorption. In the intraband case there is promotion from the $1 S_{\mathrm{e}}$ to the $1 \mathrm{P}_{\mathrm{e}}$ levels of the confined system. The key potential of this is that the material generates a narrowband absorption at some energy lower than the bandgap and as such only incident photons that are resonant with the intraband feature are absorbed, in contrast to interband features that absorb at all energies above the bandgap. The downside is that the doping of each dot, i.e. the population of the $1 S_{e}$ must be close to its degeneracy, 2 in the case of the $1 S_{e}$ level. Populations higher or lower than the level degeneracy will result in dark current between unfilled $1 S_{p}$ and $1 S_{e}$ states respectively that will obscure any photocurrent. This was first demonstrated by Deng et al. in 2014 [112], and has since been extended to the LWIR with a room temperature responsivity of $0.8 \mathrm{~A} \mathrm{~W}^{-1}$ reported [113]. Most recently $\mathrm{HgSe}$ has been used on a plasmonically activated substrate with resonance peaks matched to the centre of the narrowband emission curves. This reduced the absorption peak widths and as such increases the potential colour sensitivity of the device [114].

\subsection{Summary}

The aim of this work is to lever the solution processability, and the inherent quick prototyping this allows, to fabricate PC QD devices that display 
metrics that are competitive with the current state of the art QD devices. Throughout, the focus will be on maximising simplicity and exploring materials, deposition methods and substrate materials in order to create a low cost template for QD device design. The data Chapters cover the following areas:

- Chapter 2 gives background information on the equipment and processes used to synthesise the QDs, fabricate the devices and characterise both the QDs and the devices. Particular focus is placed on the experimental set ups that were built for this work and which give the majority of device data presented in the data chapters.

- Chapter 3 details the steps along the way to, and the production of, a flexible detector that uses all air processed QDs to deliver a very competitive responsivity whilst also demonstrating that the band edge of the CQDs are preserved in the electrical response of the device.

- Chapter 4 explores, in depth, the electrical hysteresis that develops in air exposed devices. It examines the reason for the observed behaviour and goes on to demonstrate that an electrically symmetrical QD device can display memory characteristics through charge trapping.

- Chapter 5 takes the premise of Chapter 3 and pushes it to the limit with the equipment available. It is shown that an array of $15 \mathrm{sub} \mathrm{mm}$ pixels can be reliably fabricated with spray coating and that they can offer spatial image information without a lens system or shielding. More importantly it is demonstrated that charge trapping enables photogain in these pixels and that this allows detection of targets that are only $75{ }^{\circ} \mathrm{C}$ hotter than the device itself. The EQE for the best performing devices is calculated to be $>20$ which is currently an order of magnitude greater than any other reports. 
- Chapter 6 explores the novel electrical behaviour observed in tin chalcogenide NCs when measured under IR illumination. The devices do not operate in a conventional PC mode but instead show hopping conduction that is mediated by a surface species that interacts with MIR radiation. 


\section{Chapter 2}

\section{Methods}

The methods used in the completion of this work can be broadly broken down into methods of fabrication, methods used to characterise the QDs and methods used to determine the electrical performance of the devices. Where required some data is used here to illustrate certain design methods.

In this Chapter pre-existing techniques and equipment will only be discussed briefly. More explanation is given for the processes and experimental set ups that were designed and assembled by the author solely to facilitate this work.

\subsection{Fabrication}

\subsubsection{QD Synthesis}

Variations of the injection method were used for all QD syntheses in this work [84]. Three neck round bottom flasks were held above a magnetic stirrer and a temperature controlled mantle was raised until in contact with the flask. One neck held a glass temperature probe holder, and into this a temperature probe was placed and this gave a reading to the temperature controller. One neck was attached to the Schlenk line and the third 
neck was capped with a rubber septa to allow injection. This general set up is shown in Figure 2.1.

\section{To Schlenk line}

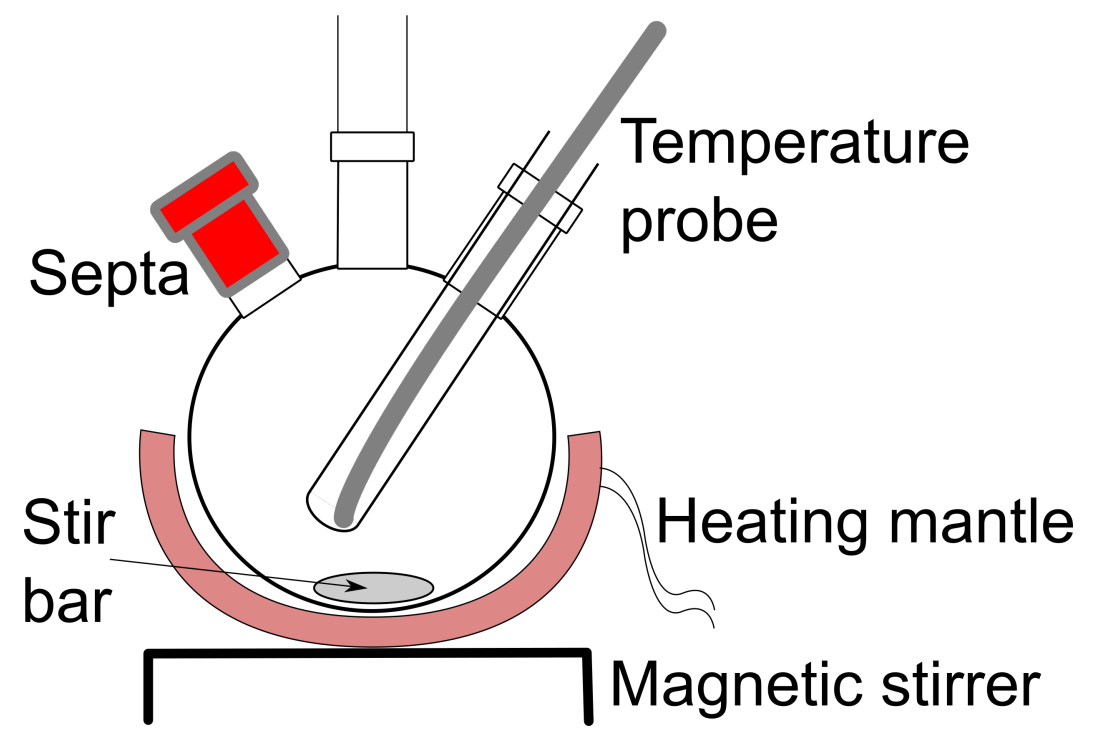

Figure 2.1: The generic setup used for $Q D$ synthesis. The initial precursor is placed into the flask with a (generally) coordinating solvent, heated to the required temperature and gently spun for uniform mixing. The Schlenk line allows the solution to be degassed and then held under vacuum, removing oxygen and water from the reaction.

Schlenk Line The round bottom flask was attached to a Schlenk line that contained a vacuum line and a nitrogen line. The vacuum line was operated at a pressure of 0.02 mbar as measured by a pirani gauge mounted on the line. The vacuum was provided by a rotary vacuum pump that passed through a liquid nitrogen cold trap before entering the main vacuum line. The nitrogen was the house supply and was flow rate controlled by two in line controllable valves. The flow rate was held at $0.021 \mathrm{~min}^{-1}$. Air ingress at negative pressure was prevented by a silicon oil bubbler.

Glovebox Dangerous, air and water sensitive compounds were handled and prepared in a chemical glovebox with solvent still. The glovebox held 
an analytical balance for sample prep along with a micro pipette and other basic measurement equipment.

Centrifugation Sedimentary centrifugation was required for a number of the chemical preparations and was done on either a large (up to $50 \mathrm{ml}$ tube volume) machine with a maximum rpm of 10000 or an eppendorf machine for smaller samples with a maximum RPM of 14400, both machines were in air.

\subsubsection{Photolithography}

All devices used in this work were fabricated on site by the author. Optical UV photolithography as detailed below is a very common and relatively simple process used for fabrication of features on the micron scale [115]. The process used here involves the use a positive resist. This class of resists remain insoluble to a developer unless they have been exposed to UV light. Following UV light exposure the exposed areas become soluble and can be easily removed from the substrate. This allows the transfer of patterns on optical stencils (masks) to a substrate that then has another material deposited onto the exposed areas of the surface. Remaining unexposed resist is then removed by organic solvents. The one step method uses a mask that directly exposes the resist to a positive image of the required device.

1. The initial step is to clean the substrate and then spin coat on the liquid photoresist. Following this the resist is heated on a hot plate in order to drive out any solvent and form a solid, homogeneous layer, Figure 2.2 A.

2. The resist coated substrate is placed underneath, and just in contact with the mask. The featured side of the mask must be closest to the resist layer so as to reduce any diffractive effects that may cause the exposed area to differ from the equivalent area on the mask. Once 


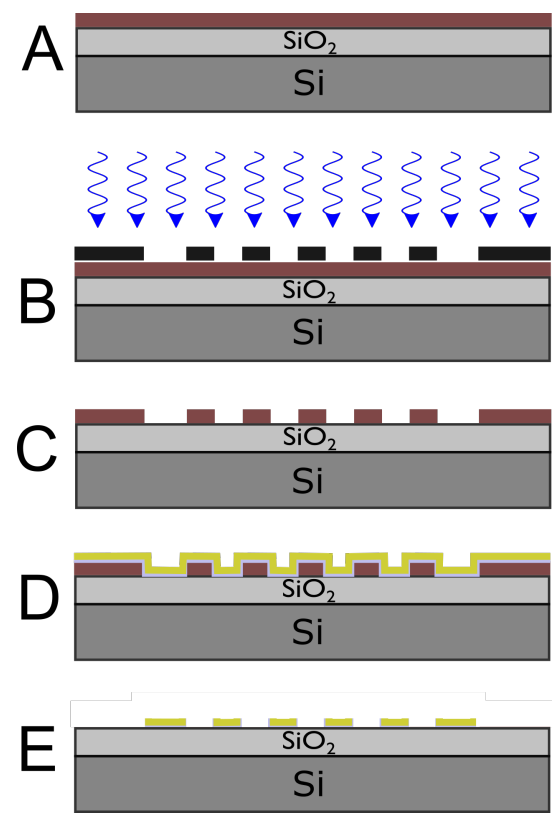

Figure 2.2: The main steps of the photolithographic process, detailed in the text

in place the mask is illuminated with UV light. The exact exposure time required is determined by the resist composition and thickness, Figure 2.2 B.

3. Once exposed the substrate is placed in the developer solution until the exposed areas are fully removed. At this point the device can be examined with an optical microscope to determine if the exposure and development was successful. The substrate should now only have resist left on the areas that are not the final device structure, Figure 2.2 C.

4. The substrate has a $\mathrm{Cr} / \mathrm{Au}$ layer evaporated, which covers both the remaining resist and the exposed areas, Figure 2.2 D.

5. The substrate is submersed in an organic solvent which breaks down the remaining resist. Removing the $\mathrm{Au}$ from all areas that were not bare substrate, this leaves the desired device pattern in $\mathrm{Cr} / \mathrm{Au}$ on the substrate, Figure 2.2 E. 
Mask Design The masks used to fabricate the devices used in this work were designed in Layout Editor and then commercially fabricated off site. The device design requirements and details will be covered in the experimental Chapters.

\subsubsection{Device Solution Processing}

There were a number of solution deposition methods available to pattern the QD solutions onto the device substrates.

Spin Coating Spin coating involves a rotating chuck that uses vacuum suction to hold onto a substrate. The solution is then dropped onto the substrate either before or during the spin period [115]. There are a large number of parameters available, such as acceleration of the chuck, however the two most commonly varied are final spin speed and duration. The basic principle is that the faster the spin speed the thinner the final layer of the solution. Duration is generally set somewhere between 30 and $60 \mathrm{~s}$ however factors such as the viscosity of the solution and solvent evaporation should be taken into account when trialling speed and duration settings. Clearly this is a useful technique when the desired outcome is the complete coverage of a substrate with a uniform homogeneous layer of a single solution, such as coating photoresist.

Spray Coating Spray coating is the deposition of any material via an aerosol method. A solution is mixed with a stream of gas and then ejected from the device within the gas stream. The major benefit is that the aerosol can be accurately targeted and as such allows for accurate spatial deposition. For solutions there is the added complication that spray coating allows a choice of how much of the solvent is transferred to the target. For a given solvent, reducing the range will increase the concentration on the target and increasing the range will reduce the concentration. For low boiling point solvents there will be a range at which all of the solvent has 
evaporated during travel and only the suspended particles will hit the target. Due to the exposure of the particles to the flow gas it is best to use inert gasses in these systems.

Drop Casting The simplest method by which to transfer a solution is to drop it. The benefits of this method are that it allows a known volume of solution to be repeatedly used and also allows spatial control. Inkjet printing is low volume drop casting with a high accuracy printhead. The downside to this method arises from the evaporation of the solvent from the drop. For larger drops $(>\mu \mathrm{l})$ as the solvent dries it does so from the outside of the drop and causes a "coffee stain" effect of deposited particles. This enhances particle aggregation and reduces homogeneity. It also reduces spatial control of the drop. For smaller volumes of solution this effect is less pronounced. Drop casting can be optimised via solvent choice that aims to give a controlled drying rate, although the application of this can be offset by lower stability of the NCs in the new solvent blend.

\subsection{Characterisation}

\subsubsection{IR Absorbance Measurements}

Arguably the most important instrument in this work was the Bruker Tensor T-27 Fourier Transform Infrared (FTIR) spectrometer as this allows the absorption of materials throughout the MIR and LWIR to be examined. FTIR spectroscopy differs from dispersive spectroscopy, that uses a monochromatic light source, by the use of an IR blackbody (BB) source that is spectrally broad. This light is then modulated in an interferometer generating a number of unique spectral distributions that serve as the light probes to the sample [116]. The absorption of each spectrum is then recorded at the detector. To return the final absorption or transmission spectrum, the absorption recorded for each known spectral selection is 
used along with the known wavelength composition. This is a mathematical process that is achieved using a Fourier transform, hence the name. When looking at colloids or liquids the work presented here used an attenuated total reflectance (ATR) attachment. This uses the nature of the evanescent wave at the point of total internal reflection within a medium, to probe samples without requiring a path length through the material. As such this allows rapid and simple characterisation of samples. When absolute values or solid materials (such as deposited films) need to be examined a transmission holder was used in place of the ATR attachment. This method is complicated by the transparency of any substrate over the IR region being examined. Measurement of the absorption in the NIR and SWIR $(1.0-2.5 \mu \mathrm{m})$ is not possible with either a typical FTIR or UV-VIS spectrometer and requires a specific NIR instrument. There was a Bruker Vertex 80V VIS-NIR-MIR available for this work and this allowed a measurement from the LWIR into the visible region. It could only function with a transmission holder, however it did have the ability to pump down the sample chamber and measure the sample in vacuum, thus removing atmospheric noise from sample measurements.

\subsubsection{Transmission Electron Microscopy}

Transmission Electron Microscopy (TEM) is similar to all electron microscopy in that it utilises the lower de Broglie wavelength of electrons to image features that are smaller than that achievable with an optical microscope [117]. This is due to the Abbe diffraction limit which states that the angular resolution of a system has a fundamental limit that is proportional to the wavelength being observed. As an example the de Broglie wavelength for a $1.5 \mathrm{eV}$ photon is $827 \mathrm{~nm}$. This is compared to an electron accelerated by a $200 \mathrm{keV}$ accelerating voltage which has a wavelength of $2.74 \mathrm{pm}$. This difference in the smallest possible feature size is the reason that electron microscopes can directly image at the nanoscale. 
TEM, specifically, involves the electron beam passing through the sample and then being focussed onto an image plane, that is now commonly a digital camera. Thus the key constraint with TEM work is that the beam must pass through the sample and substrate, placing a restriction on the in plane depth of the sample. This makes TEM highly suitable for imaging nano materials as they are "thin" enough that there is no issue with beam transmission through the material. The greyscale contrast of the obtained images is dictated by the sample thickness and elements being studied. Generally this means that lighter elements are difficult to image and heavier elements show up clearly. Due to these fundamentals TEM is an excellent method for the the study of semiconductor nanomaterials. This direct imaging can give information not only about the in plane shape and size but also about the structure of the material, for example the lattice planes of crystalline samples can be directly imaged. If more accurate information about the crystallinity of the sample is required the TEM can be operated in the selected area electron diffraction (SAED) mode. In this mode the low wavelength of the electron beam compared to the lattice spacing results in the electron beam being diffracted, which creates a diffraction pattern at the image plane. For a single orientation of a highly ordered crystal this will create a series of dots, with the spacing between them relating to the inter plane spacing of various lattices vectors. For a disordered collection of crystalline samples, such as a distribution of semiconductor QDs then the image will consist of ring patterns [117]. The TEMs used in this work were a JEOL 2100 and a JEOL 2010.

Energy Dispersive X-Ray Spectroscopy Energy Dispersive X-Ray Spectroscopy (EDS) is a means of conducting elemental analysis of a sample [16]. The underlying principle is based on the fact that every element has a unique set of energy levels, and that the relative energy of the filled levels close to the nucleus are unperturbed by local bonding. High energy particles or X-rays will excite electrons from the inner levels of an atom 
leaving unfilled holes. This hole will be filled by an electron from a higher level looking to lower its energy. The energy difference between the level of the filling electron and the hole is emitted as a photon in the X-ray region. As this energy is the difference between two normally filled levels of the atom it will be characteristic of that element and as such the detection of the energy and number of these emitted characteristic $X$-rays gives information about the elemental composition of the sample. This functionality is added to a TEM by the addition of an X-ray detector within the column, the high energy particles being provided by the image beam. Operating the TEM in the conventional focus mode, with a wide beam focused through the sample area onto the image plane, allows the EDS spectrum of the entire area being imaged to be obtained. However no spatial information about the elemental composition is contained in this data.

Scanning Transmission Electron Microscopy Scanning TEM (STEM) uses the same basic principles of operation as a TEM however the spot size of the electron beam is now small compared to the sample being imaged [117]. This small spot is rastered across the sample area that is being imaged. The major advantage of STEM imaging is that there is direct spatial correlation between areas of the sample and the beam. Therefore any interaction with the beam and the sample can be spatially mapped. This is most commonly used with an EDS detector as the combination of these allows high resolution elemental maps to be obtained. This allows the location of individual elements within the sample area to be obtained. The map can then be compared to the STEM image to give a large amount of information about a sample.

TEM Sample Preparation TEM samples are prepared on very thin polymer (Formvar) films on an underlying supportive copper grid, the polymer side of which is carbon coated. The thickness of these two layers that are imaged through is in the region of $20-50 \mathrm{~nm}$. This 
carbon/polymer layer provides the physical support and homogeneous contrast image background for the samples to be dispersed upon. Low concentration colloids must then be prepared in solvents that will not weaken the polymer substrate. A small amount $(\approx \mu \mathrm{l})$ is dropped onto the grid and allowed to dry. For best results this grid can be stored in vacuum. Oxygen plasma cleaning immediately prior to use can assist in removing any organic material that would otherwise negatively affect imaging.

\subsubsection{Scanning Electron Microscopy}

Scanning Electron Microscopy (SEM) refers to an imaging methodology that does not require transmission through the sample, and as such can provide information about any type or preparation of material. Instead of imaging the transmitted electron beam the SEM scans a focussed beam across the sample [16]. The interaction between the beam and the sample causes the emission/scattering of various electron energies that can be picked up by specific detectors. The detected particle characteristics are then attributed to the beam focus point and images are created. The most common imaging mode for SEMs is the detection of secondary electrons, these are electrons from the material that are ejected from the innermost shell of the atoms on the surface due to inelastic scattering with beam electrons. They have a relatively low energy and as such the only electrons in this energy range that can be detected are those from atoms within a few $\mathrm{nm}$ of the sample surface. Consequentially they provide excellent topographical information about the surface being imaged. Imaging is also frequently done with backscattered electrons. Which are beam electrons that have been elastically scattered by the sample. They do not provide as accurate topographical information as they can be detected from a larger depth within the sample. However as the elastic scattering intensity is dependent upon atomic mass these electrons provide a contrast depen- 
dent upon atomic weight and as such offer spatial information about the composition and density of the sample. The use of characteristic X-ray detection for EDS within SEMs is methodologically identical to EDS within TEMs however due to the scanning nature of the beam they will return compositional maps of the sample under all operating conditions. The SEM used in this work was a JEOL 6500F.

SEM Sample Preparation As it is far easier to get a sample into an SEM than a TEM more care must be taken to ensure that the sample is properly prepared. The major issues are for samples that are not highly conductive. In this case the electron beam causes charging in areas that cannot dissipate the electrons from the beam. Charged areas strongly perturb the beam behaviour and cause defects with the image. To prevent this, samples are mounted to aluminium studs with conductive carbon tape. The tape should be placed as close to the intended image area as possible without affecting the sample. If this is not sufficient samples can be completely coated with either carbon or platinum, very thin layers of which cover the surface of the sample and allow beam electrons to ground to the stud without affecting the topographical or compositional information of the sample. Following these steps the samples are left in a vacuum desiccator for at least $12 \mathrm{~h}$, which prevents the samples and carbon tape from out-gassing inside the machine.

\subsubsection{X-ray Diffraction}

X-ray Diffraction (XRD) refers to a technique whereby a diffraction pattern is generated by reflection from the lattice planes of a crystalline sample [16]. The key determinant here is that the wavelength of the incident wave is similar to the periodic spacing of the material that generates the pattern. X-rays have a wavelength that is similar to the lattice spacing of crystalline samples (Angstroms) and this is why they are used in this application. Bragg's Law states the relationship between the crystal spacing, 
the incidence angle and the wavelength of the incident light. Constructive interference will occur when the path difference is an integer number of wavelengths. As the wavelength and the angle is known the lattice spacing can be found. Most commonly the sample is a powder or some other randomly orientated collection of the material. Thus the output data will consist of an intensity of measured radiation and an angle that, averaged over numerous runs will provide a unique result for a material. Due to the powder nature of the sample it is assumed that all lattice spacings will be detected and measured. The XRD machine operates by first collimating a monochromated $X$-ray source and then measuring the reflection of this beam from a powder sample that is usually rotating. The data is then compared against a library of spectra to obtain information about the composition and lattice parameters of the sample. The XRD equipment used for this work was a Panalytical XRD using $\mathrm{Cu} \mathrm{K} \alpha$ radiation.

\subsubsection{Parameter Analyser}

For electrical measurements a Keithley SCS-4200 Parameter Analyser (PA) was used. This is an integrated system that comprises two source measurement units (SMUs) that can be independently tasked using the inbuilt software. Connections between the SMUs and the device under test (DUT) were custom made and co-axially cabled. In this configuration the PA could accurately measure signals down to $500 \mathrm{pA}$. Using the PA software the SMUs could easily be programmed to operate in either a sweeping mode where a voltage source is swept between two values and the resultant current through the device is recorded, or in a sampling mode where a constant voltage source is applied and the resultant current is recorded over time. The minimum effective sampling time in this mode was found to be on the order of $15 \mathrm{~ms}$. As such if higher frequency resolution was needed an oscilloscope would be required. For both of these operating modes the SMUs can also drive at a set current and measure the voltage 
response.

\subsubsection{Opto-Electrical Measurements}

As part of this research project the equipment required for electro-optical measurements in the MIR had to be selected, purchased and assembled. The final measurement set up consisted of a blackbody (BB) source, a series of IR longpass filters, a power meter (PM) for calibration, an optical chopper and an optical cryostat with electrical connections to the PA. This is shown in Figure 2.3.
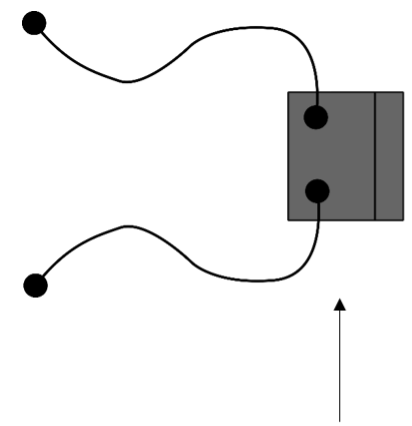

DUT or power meter windows $\times 2$
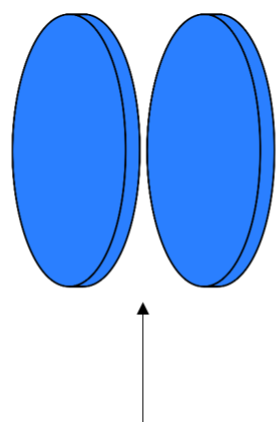

Sapphire
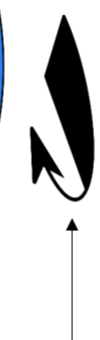

Chopper

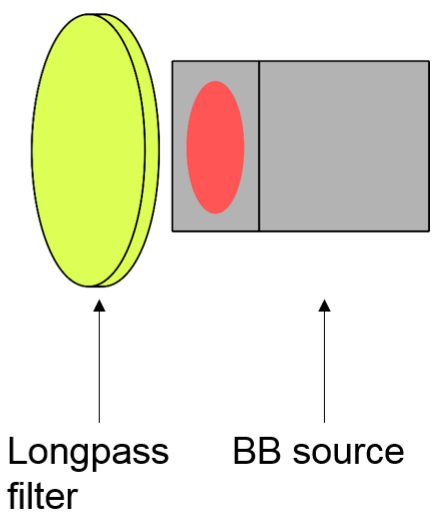

Figure 2.3: Cartoon showing the standard optical path used in MIR device testing. The filter and chopper are in air and the sapphire windows are part of the cryostat.

Blackbody Source The only viable source for generating wavelengths in the MIR was an Omega BB-4A blackbody source. These are optical sources that are constructed so as to have a spectral emission that is defined by the Planck function with a high emissivity value [17]. It operates by holding a cavity at some uniform temperature where the radiation inside the cavity depends only upon this temperature. An ideal BB cavity must have walls that have emission of radiation in equilibrium with the radiation absorbed by the walls. In practice the need for an opening means that the source cannot be perfect, however modern designs are excellent and the BB used 
in this work has an emissivity of 0.99 over the temperature range 100-900 ${ }^{\circ} \mathrm{C}(373-1173 \mathrm{~K})$. The diameter of the cavity opening was $21.56 \mathrm{~mm}$.

Longpass Filters Unlike the visible and NIR regions where monochromated sources are commonplace, the MIR region has fewer options for spectrally selecting incident signals. The method chosen for this project was the use of optical longpass filters to cut out higher energy radiation and give MIR only signals. The filters used cut-on at $1.65 \mu \mathrm{m}, 2.4 \mu \mathrm{m}, 3$ $\mu \mathrm{m}$ and $4.08 \mu \mathrm{m}$. The spectral transmission of each of these filters as provided by the manufacturer is given in Figure 2.4. The validity of this data was confirmed by measuring the filters in the FTIR in path mode. Each filter was mounted in a $25.4 \mathrm{~mm}$ optical lens holder and this was placed directly in front of the BB cavity opening. The path between the filter was covered to reduce noise from external sources. The filters are not lenses and as such do not affect the radiometric quantisation of the system.

Cryostat In order to look at device performance below room temperature a Janis VNF-100 liquid nitrogen optical cryostat was used. Temperature was controlled by liquid nitrogen flowing through a needle valve and two heating elements/thermocouples, one in the sample mount and the other positioned immediately after the needle valve. The optical path between the exterior of the cryostat and the sample chamber consisted of two sapphire windows and $10 \mathrm{~mm}$ of shroud vacuum. For electrical measurements a bespoke three terminal sample mount was constructed on top of the the thermally monitored sample mount. Sample connections were wired to BNC feedthroughs that allowed the PA to be directly connected to the sample mount. The temperature outputs went to a temperature controller that was precise to $1 \mathrm{mK}$.

Radiant Power Calculation The radiant flux that is ultimately incident on the sample ( $\Phi_{d}$ having a unit of watts) is vital in assessing the perfor- 


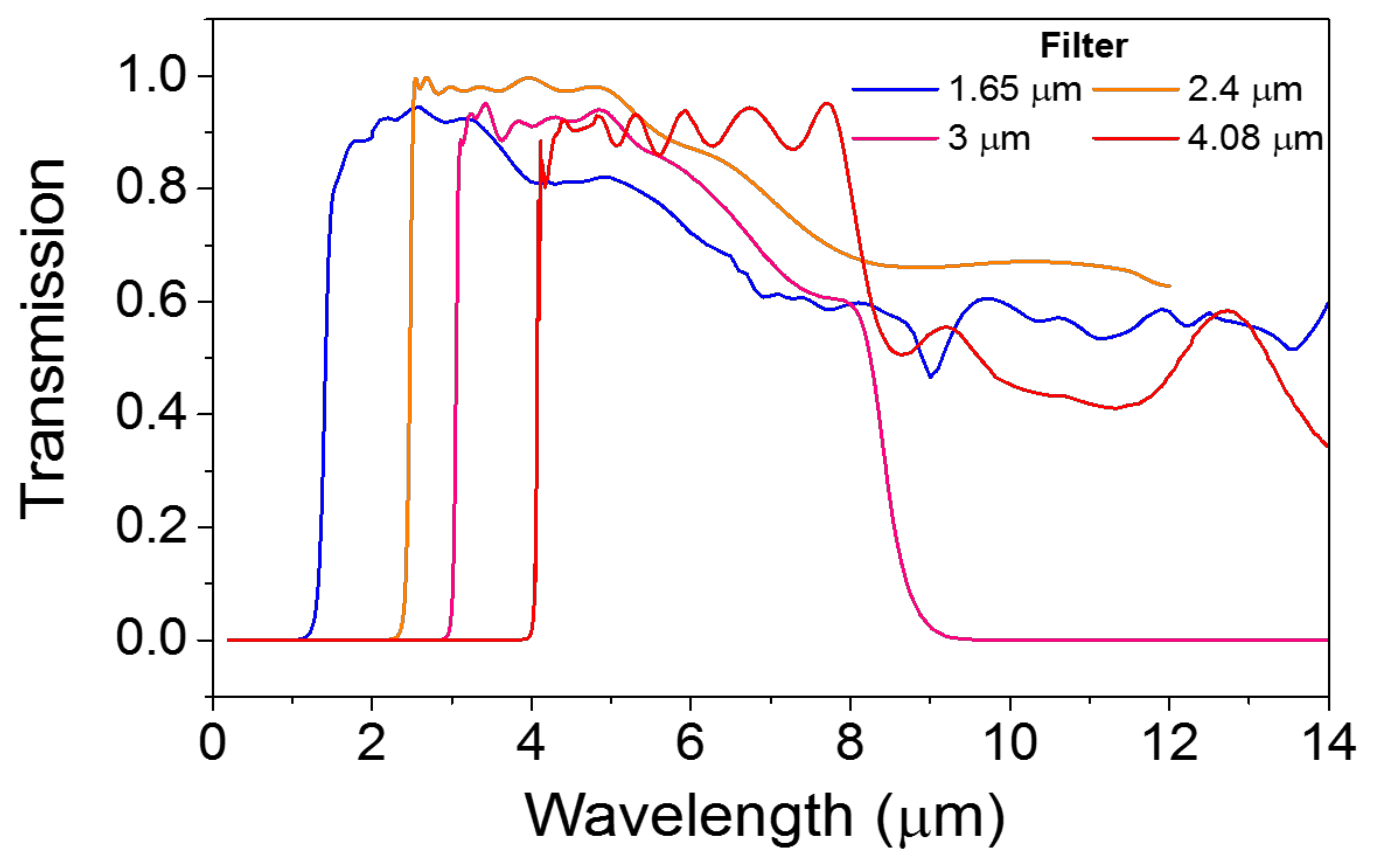

Figure 2.4: Transmission of the longpass filters used in this work, these are plotted using data points provided by the manufacturer. Each filter was tested using an FTIR to confirm the manufacturers data and the transmission spectra collected experimentally were used for calculations.

mance of any detector. The setup used here is described as an extended lambertian source and extended detector with no tilt problem within radiometry. That is to say that the BB cavity opening is treated as a non-point object, having a constant radiance that is independent of viewing angle, the detector cannot be treated as a point and the centre line of the system is perpendicular to both surfaces $[4,17,118]$. The geometric solution that gives $\Phi_{d}$ in these systems is given in equation 2.1 and is shown in Figure $2.5[4]$ :

$$
\Phi_{d}=L \Omega_{s} A_{d}
$$

where $L$ is the total source radiance with units of $\mathrm{W} \mathrm{sr}^{-1} \mathrm{~m}^{-2}, \Omega_{s}$ is the solid angle of the source as seen by the detector with units of steradians 


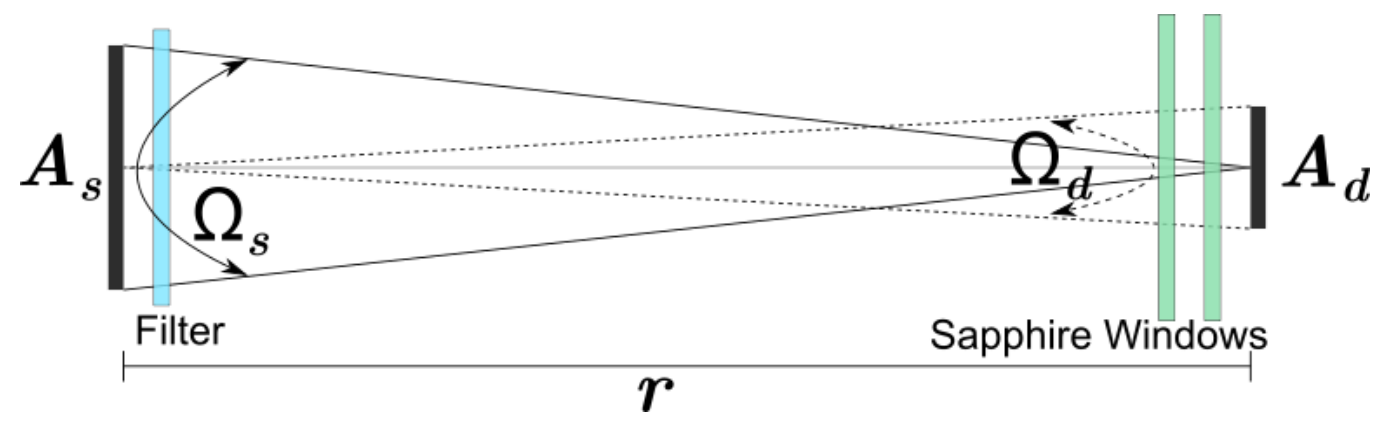

Figure 2.5: Optical path as described in the text. It is important to note that as the filter has a larger diameter than the source it does not change the optics of the system, only the transmission characteristics. The same is true for the cryostat windows.

(sr) and $A_{d}$ is the area of the detector. The source radiance $(L)$ can be obtained by integrating the Planck Law over the range of wavelengths that are incident on the sample and the transmission of the path. The total absorbed incident radiant flux is thus obtained with equation 2.2 [4,17],

$$
\Phi_{d}=\Omega_{s} A_{d} \int_{\lambda_{a}}^{\lambda_{b}} T(\lambda) \frac{2 \pi h c^{2}}{\lambda^{5}} \frac{1}{e^{\frac{h c}{\lambda k T}}-1} d \lambda
$$

where the limits of integration are defined by the system. The lower limit is $200 \mathrm{~nm}$ as this is the highest energy to which the transmission functions of the equipment used are defined. The use of $200 \mathrm{~nm}$ as the lower limit is valid as BB spectra at low temperature have negligible emission in this spectral region, this is not temperature dependent. The cut on behaviour of any filters is included in their spectral transmission, not by using some arbitrary value as the the lower limit in the integration. The upper limit is the experimentally determined bandedge of the device. $T(\lambda)$ is the transmission of the path between the source and the detector as a function of the wavelength. For this work this will be calculated numerically per nm, which is a valid solution so long as the source spectral radiance is given in the units of $\mathrm{W} \mathrm{sr}^{-1} \mathrm{~m}^{-2} \mathrm{~nm}^{-1}$. This methodology is shown pictorially in Figure 2.6. Using this method the only remaining variable is $\Omega_{s}$, which can be experimentally calculated by using a PM at the same position as the de- 

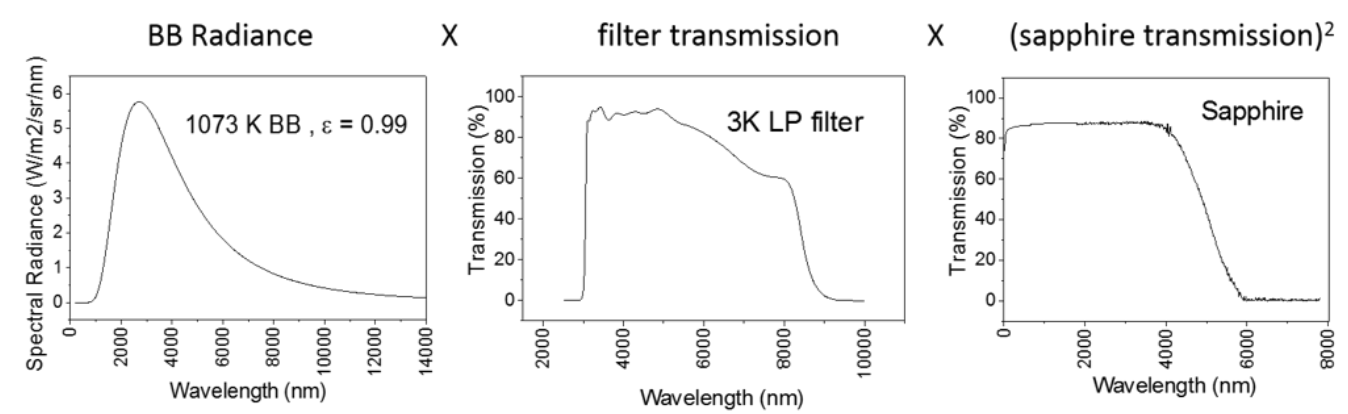

$=$ Sample Radiance

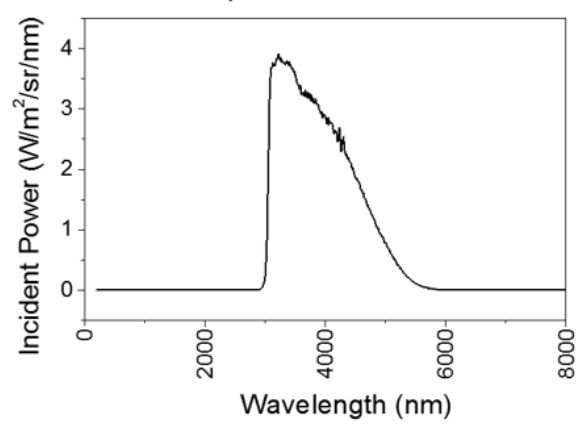

Figure 2.6: Pictoral representation of the spectral transmission through the optical system $(T(\lambda))$ as used in Equations 2.2 and 2.4.

vice will occupy. Note that the PM will also have a wavelength dependent absorbance profile and this is factored into the analysis. As the radiance and PM area are known, using a variety of longpass filters (thus varying $L$ ) allows an experimentally determined $\Omega_{s}$. $\Omega_{s}$ can also be geometrically calculated from the definition that a full spherical surface subtends $4 \pi \mathrm{sr}$ of solid angle from a point inside the sphere. The solid angle of a source with area $A_{s}$ as seen by a detector at distance $r$ is then given by Equation $2.3[4]$ :

$$
\Omega_{s}=4 \pi \frac{A_{s}}{A_{\text {sphere }}}=4 \pi \frac{A_{s}}{4 \pi r^{2}}=\frac{A_{s}}{r^{2}}
$$

Responsivity Once the incident power is known then the responsivity can be calculated by finding the current change that occurs when the device is illuminated by that incident power. As all the devices in this work 
are $\mathrm{PC}$ this involves measuring the change in current from some non-zero dark current within some specified time interval. This differs from the PV approach which would use the short circuit current under illumination.

\subsubsection{Multi Channel Measurement System}

The later work in this thesis used an electrical test suite that could measure multiple voltages simultaneously. The physical construction of this measurement system that facilitates 16 connections to a small chip at the same time as changing the incident optical power was very involved, and will only be covered briefly here. The device side will be covered in Chapter 5 . This system was designed around a National Instruments (NI) PXIe 4138 SMU to provide a driving voltage and an NI PXIe 4303 multi-channel DAQ to collect voltage information. As a starting point the system was designed around spring loaded pins with a $0.05 \mathrm{~mm}$ head diameter that would contact the device. To allow some small degree of misalignment the device pad size was chosen to be $2 \mathrm{~mm}$ square. This was the initial starting point for the device design that is shown in Chapter 5. In order to allow a small optical path length the connection system was chosen to connect top down but then re-connect to the measurement board, thus minimising the stand off above the device. This was achieved using a 3-D printed block for pin alignment and a custom printed circuit board (PCB) that connected the device step up pins to the board step down pins, as shown in Figure 2.7 A. The NIX PXIe 4303 is a multi-channel voltage probe, therefore the connections for each line needed to provide a voltage to measure over, rather than an inline current measurement. The simplest way to achieve this is to provide a known resistance to measure the voltage over. The measurement board and device combined then form 15 individual potential dividers with a light dependent top resistance. The net diagram for this is shown in Figure 2.8 and the PCB is shown in Figure 2.7 B. The validity of this electrical set up for photoconductive detectors is best shown as a 


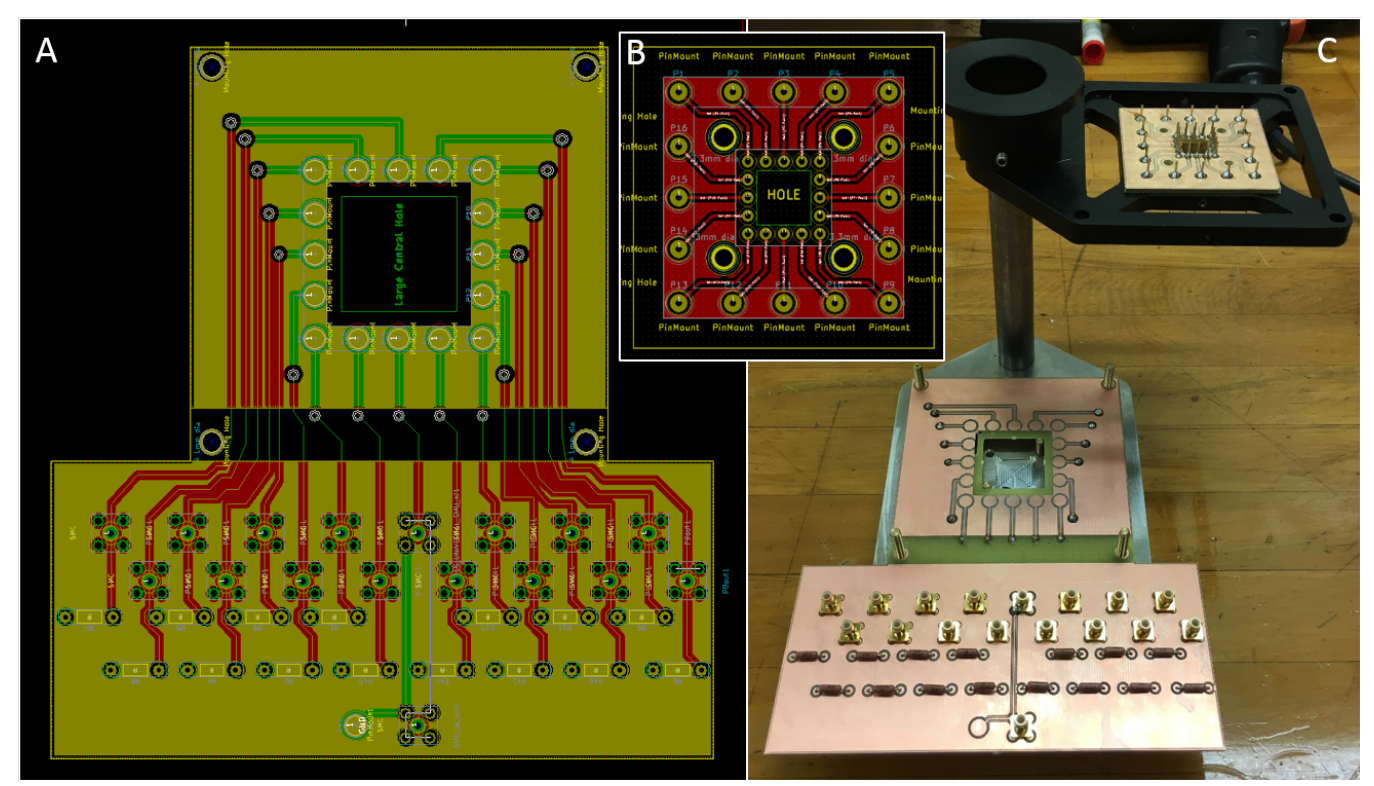

Figure 2.7: $A$. The $P C B$ design for the bottom board of the 16 channel measurement equipmeny. B. The PCB design for the top board. C. The fabricated and soldered boards in the bespoke mount.

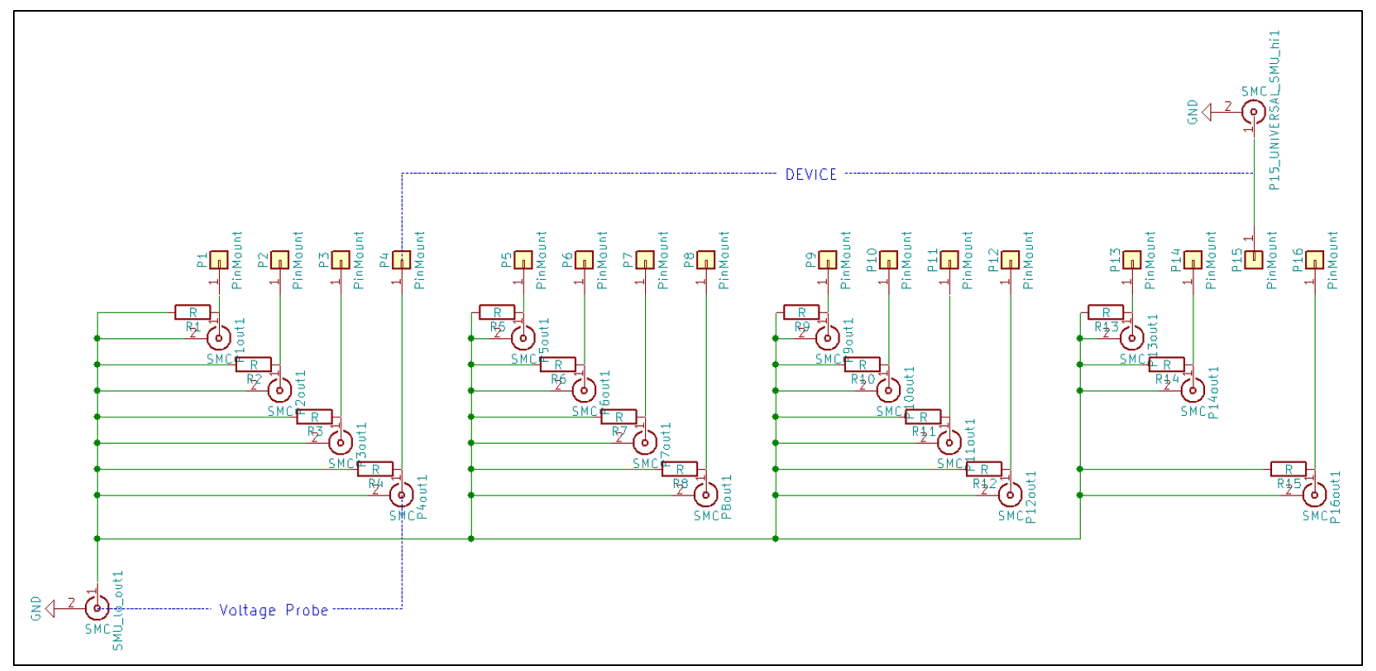

Figure 2.8: Net diagram for the bottom board. An example of one pixel and its associated voltage probe are shown in blue.

Thorlabs tutorial found at Reference [119]. 
Optical Path The optical background for this device was inherently more noisy than the previous setup due to the lack of shielding that the cryostat provided. In order to maximise the number of incident photons the path length was modified with the addition of a $25.4 \mathrm{~mm}$ diameter $\mathrm{CaF}_{2}$ plano-convex lens that approximately collimates some of the extended source from the BB. This serves to virtually move the detector closer to the source when considering the effective solid angle that is observed by the detector. This is shown in Figure 2.9. The addition

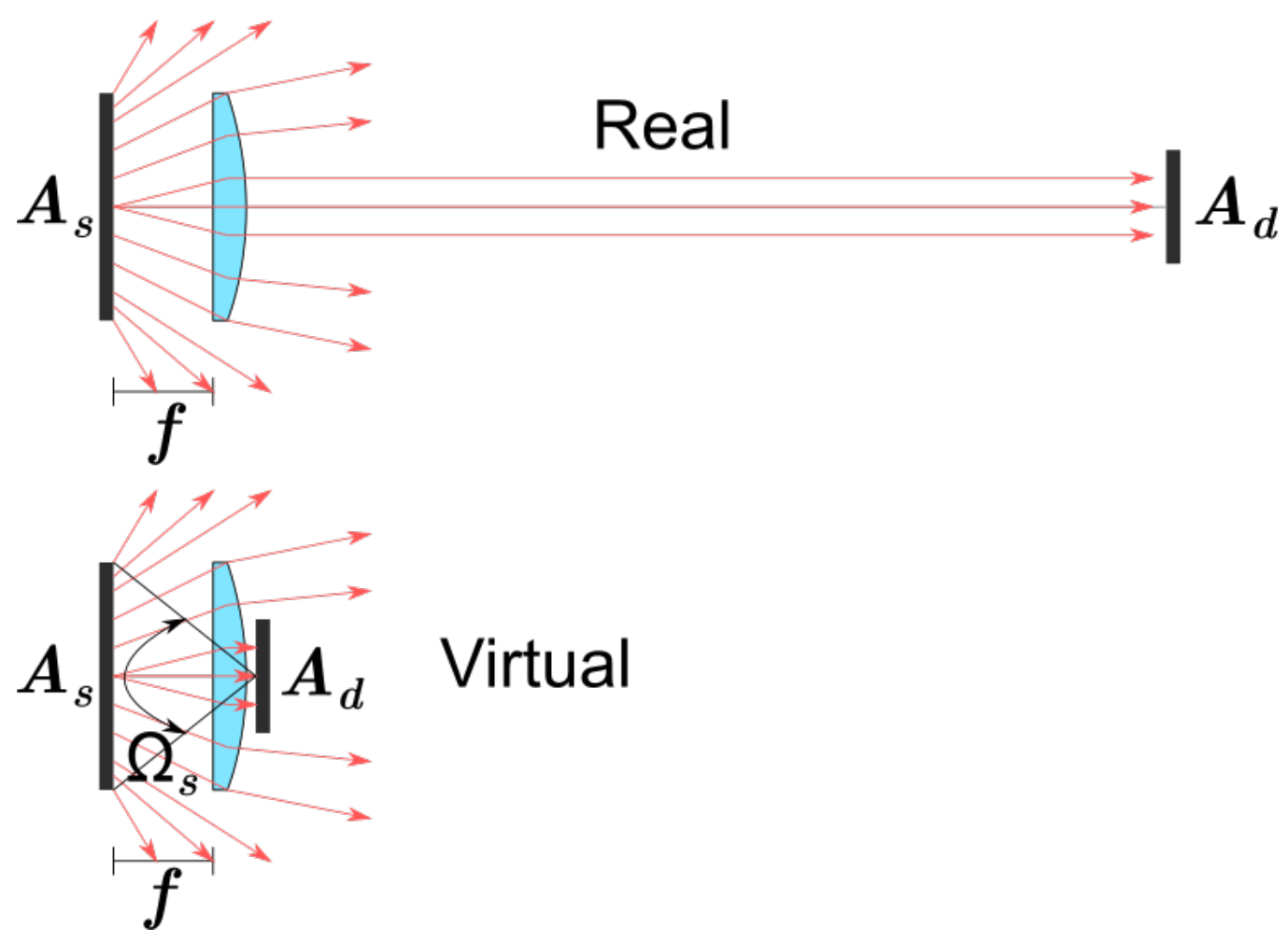

Figure 2.9: Effect of a plano-convex lens in the optical system. The approximate collimation of a proportion of the extended $B B$ source creates a higher incident power on the detector. The solid angle as seen by the detector should then be calculated from the focal length of the lens, not the actual distance of the detector from the source. This figure is not to scale.

of this lens increases the intensity at the detector by increasing the value of $\Omega_{s}$ used in Equation 2.2 relative to the case without the lens. To illustrate 
this we use Equation 2.1 with a calibrated thermal PM reading out units of $\mathrm{mW} \mathrm{cm}^{-2}$. We note that the area of the PM is larger than the area of the detector so it is a fair assumption that if this approximation is valid for the PM it will also be valid for the detector. Note also that the diameter of the lens is greater than the diameter of the BB source cavity opening. Integrating the Planck power law over the path, i.e, taking into account the spectral transmission of the lens and the spectral absorption of the PM, with limits of $0.2 \mu \mathrm{m}$ and $12 \mu \mathrm{m}$, the incident power irradiance is 1.3469 $\mathrm{W} \mathrm{sr}^{-1} \mathrm{~cm}^{-2}$ for a $973 \mathrm{~K} \mathrm{BB}$ source temperature. The transmission of $\mathrm{CaF}_{2}$ drops to zero beyond $11 \mu \mathrm{m}$ so these limits do accurately model the system. Using Equation 2.3 with $r$ as the focal length of $10 \mathrm{~cm}$ and $A_{s}$ as $3.65 \mathrm{~cm}^{2}$ gives an $\Omega_{s}$ of 0.0365 , which when combined with the incident power radiance above gives $\Phi_{d}=49.2 \mathrm{~mW}$. The measured value is $50 \pm 0.5 \mathrm{~mW}$. Without the lens in place the measured value is $37 \pm 0.5 \mathrm{~mW}$ so the lens system is functioning as intended. When calculating flux the solid angle used will be the experimentally determined value from the power calibration, $\Omega_{s}=0.0371 \pm 1 \%$. In general it is better to over estimate the flux and therefore underestimate responsivity and other important values, than underestimate flux and provide inflated metrics. Chapter 5 also deals with the concept of EQE instead of responsivity. The same methodology regarding path transmission as illustrated above is valid but instead of using Equation 2.2, giving radiant flux in watts at the detector you must instead use the expression for spectral photon radiance with wavelength $L_{\lambda}^{P}$. This is shown at Equation 2.4, giving the photons per second at the detector $[17,43]$ :

$$
\Phi_{d}^{P}=\Omega_{s} A_{d} \int_{\lambda_{a}}^{\lambda_{b}} T(\lambda) \frac{2 c}{\lambda^{4}} \frac{1}{e^{\frac{h c}{\lambda k T}}-1} d \lambda
$$

It is worth noting that due to the different wavelength dependence in Equations 2.2 and 2.4 the wavelength of peak emission in watts is at a different wavelength to the peak emission in photons, although the total 
energy radiated at each wavelength is constant.

The results in this thesis rely on a large number of analytically performed integrations to give total flux, either in power or photons per second, over a specific spectral transmission range. If the reader wishes to get a feel for these calculations please see Reference [120]. 


\section{Chapter 3}

\section{HgTe Synthesis and MIR Detection}

Parts of this work are published in:

$300 \mathrm{~nm}$ Spectral Resolution in the Mid-Infrared with Robust, High Responsivity Flexible Colloidal Quantum Dot Devices at Room Temperature, Cryer, M. E.; Halpert, J. E. ACS Photonics, 2018.

\subsection{Motivation}

Although recent progress in colloidal nanocrystal (NC) photodetectors has seen performance that has begun to compete with commercially available MIR and LWIR detectors the work reported in this Chapter focuses not on outperforming high-cost cooled detectors, and instead levers the unique solution processability and size selection afforded by colloidal QDs to focus on devices that can outperform the current crop of low cost, thermal detectors. This is arguably an effort to which colloidal QDs are more suited. Currently, commercial demand for cheap detectors is almost entirely met by microbolometers, which thermally detect in the LWIR and meet the demand from a growing market that is estimated to be worth 
$\$ 1.6 \mathrm{~B}$ by $2022[1,2]$. This growth is expected to come from an increased demand from industries such as autonomous transport, firefighting, security screening, environmental monitoring and a larger general civil uptake $[8,11,12,121]$. The unit cost for a sensor to be competitive is expected to be less than $\$ 10[1,2]$ and although MIR detectors fabricated using epitaxial growth techniques can outperform microbolometers in imaging metrics, they are very expensive and are very unlikely to ever compete with them on price $[2,122-124]$. As such there is a real demand for low cost, MIR detectors that can operate at room temperature with superior performance to microbolometers.

\subsection{Background}

Colloidal NCs in the form of HgTe quantum dots (QDs) have been used for room temperature MIR detection since 2011 [43]. Sandwich-style device architectures using these materials have achieved background limited performance [54], an important milestone as this shows that the dominant device noise is caused by the variation in background signal rather than any inherent device behaviour. Inorganic ligand exchange has been used to drastically increase performance at room temperature [99]. Phototransistors have demonstrated gate tunable, high speed performance [110] and HgTe QDs have been used to sensitise transistor structures [109,125]. Together these show that colloidal HgTe NC devices can now offer room temperature detection with metrics that are competitive with cryogenically cooled epitaxial materials [54,99, 109,110, 125,126].

There are two key benefits to using colloidal NCs for photodetection. The first of these is bandgap tunability via size tuning. Chemical control of HgTe NC synthesis permits tuning the bandgaps that span the entire infrared through size tuning $[94,97,98,127,128]$. This allows spectrally selective pixels that can operate in the visible [129], near- [130] mid- and 
long wave IR, including multi-spectral devices [111], and can therefore replace the complex colour filters used in commercial devices $[4,131]$. The second advantage of colloidal NCs is in the device fabrication process. NC inks have been used to fabricate MIR detectors via inkjet printing of fine features [108], PDMS stamping [111], and spray coating of aqueous NCs [110]. These techniques are advantageous for producing single step patterned devices and ideal for devices employing planar structures with simple symmetrical electrodes [132]. As points of comparison we will be aiming to show imaging potential for an uncooled device at room temperature, with a refresh rate above $30 \mathrm{~Hz}$ [133] and with a specific detectivity between $1 \times 10^{9}$ and $1 \times 10^{10}$ Jones [134].

\subsection{Experimental}

\subsubsection{CQD Synthesis}

This section details the complete finalised process for the synthesis of the most monodisperse HgTe CQDs produced in this work.

Chemicals Used Tellurium powder (Sigma Aldrich, 200 mesh, 99.8 \%), trioctylphosphine ((TOP) Sigma Aldrich, $97 \%)$, mercury chloride (M and B, > 99.5\%), dodecanethiol ((DDT) Sigma Aldrich), octadecylamine ((ODA) Sigma Aldrich, 90 \%), oleylamine ((OLA) Sigma Aldrich, 70 \%), tetrachloroethylene ((TCE) Sigma Aldrich, > 99.5\%), methanol ((MeOH) Fisher, > 99.9\%), chloroform (Merck, > $99.99 \%)$, ethanol ((EtOH) Fisher, $>99.99 \%$ ), propylamine ((PPA) Sigma Aldrich, $98 \%$ ), arsenic(III) sulfide (Strem), ethanedithiol ((EDT) Sigma Aldrich), octadecanethiol ((ODT) Sigma Aldrich).

To start, $72 \mathrm{mg}$ of $\mathrm{HgCl}_{2}$ and $6 \mathrm{~g}$ of ODA are placed in a $50 \mathrm{ml}$ three neck flask with a $20 \mathrm{~mm}$ oval stir bar. Both materials are solid at room 
temperature. The flask is put on the schlenk line and placed under nitrogen, with the heating mantle set at $120{ }^{\circ} \mathrm{C}$. The initial melting process can be sped up by using a heat gun on the flask and once the ODA has melted and the stirring is even the flask can be placed under vacuum. It is then left for at least $1 \mathrm{~h}$ to degas. Once completely degassed it is placed under nitrogen and cooled to $60^{\circ} \mathrm{C}$. TOP:Te (1M) is made by dissolving $1.276 \mathrm{~g}$ Te powder in $10 \mathrm{ml}$ TOP in a glovebox. This is stirred for 24 $\mathrm{h}$ at $40{ }^{\circ} \mathrm{C}$ and forms a clear yellow solution. Prior to use it is filtered through a $0.22 \mu \mathrm{m}$ syringe filter with no visible solids left in the filter. The tellurium precursor injection is prepared by placing $10 \mathrm{ml}$ OLA in a septa vial, placing under vacuum and heating with a heat gun. This should be done until there are no longer any bubbles forming in the vial and then the atmosphere is changed to nitrogen. $0.2 \mathrm{ml}$ TOP:Te is removed from the glovebox and instantly injected into the OLA. The TOP:Te/OLA vial is put through another heating, vacuum, nitrogen cycle and the contents are transferred into a $12 \mathrm{ml}$ syringe with a $19 \mathrm{G}$ needle which is quickly placed into the septa on the flask. This should be left for at least two minutes before injection so that any oxygen that was transferred in the needle can disperse in the nitrogen atmosphere. The mixture should still be warm to the touch. To ensure minimal heating ramp time the flask temperature should be reduced to $58^{\circ} \mathrm{C}$ and the desired cook temperature should be entered into the temperature controller. For this preparation this will be between 80 and $120^{\circ} \mathrm{C}$. When the flask temperature recovers to $60{ }^{\circ} \mathrm{C}$ the TOP:Te/OLA is injected. The flask temperature may drop but the steps above should limit this. There will be no immediate nucleation. At $65{ }^{\circ} \mathrm{C}$ the nucleation temperature is reached and the mixture will begin to darken and by $70{ }^{\circ} \mathrm{C}$ it should be completely black. When the desired cook temperature is reached the mixture should be left for $5 \mathrm{~min}$. Factors such as the ramp time and evenness of cooking temperature do affect the final size dispersion, so should be optimised. The reaction is quenched by the removal of the heating mantle and the addition of $31.5 \mathrm{ml}$ TCE and 3.5 
$\mathrm{ml}$ DDT at room temperature. Following this the reaction should quickly cool to below $50{ }^{\circ} \mathrm{C}$. If the entire batch is intended for TEM imaging the DDT can be replaced with ODT, which is solid at room temperature so must be melted before being added to the TCE quench solution.

\subsubsection{CQD Purification}

The quenched growth solution should be equally divided between three $50 \mathrm{ml}$ centrifuge tubes and $20 \mathrm{ml}$ of $\mathrm{MeOH}$ is added to each tube. These are then centrifuged at $8000 \mathrm{rpm}$ for $5 \mathrm{~min}$. The supernatant should be perfectly clear and is discarded, the precipitate is dried under vacuum for 2 $\mathrm{min}$ and then re-dispersed in $3 \mathrm{ml} \mathrm{TCE}$. There will be excess organics dried in the tubes so the black QD solutions should be removed via pipette and placed together in a clean tube (which can be weighed when empty if calculating the yield). To the $9 \mathrm{ml}$ of QD solution add $18 \mathrm{ml}$ of $\mathrm{MeOH}$ and centrifuge at $10000 \mathrm{rpm}$ for $5 \mathrm{~min}$. Discard the supernatant and vacuum dry for $2 \mathrm{~min}$. The yield to this point should be 70-90 mg of QDs. For device fabrication and film characterisation the QDs should be re-dispersed in chloroform. For TEM imaging the QDs should be crashed/re-dispersed twice more, ending in hexane. Following purification the QDs formed optically clear solutions, shown in Figure 3.1.

\subsubsection{Device Design and Fabrication}

The device template used here was chosen to be a $10 \mathrm{~mm} \times 10 \mathrm{~mm}$ design with 4 inter-digitated electrode (IDE) structures per chip, essentially a simple transistor structure with a very large width. The trench length was variable between 5 and $80 \mu \mathrm{m}$. The same masks could then be used for any substrate material. The photolithographic masks used were designed in Layout Editor. 


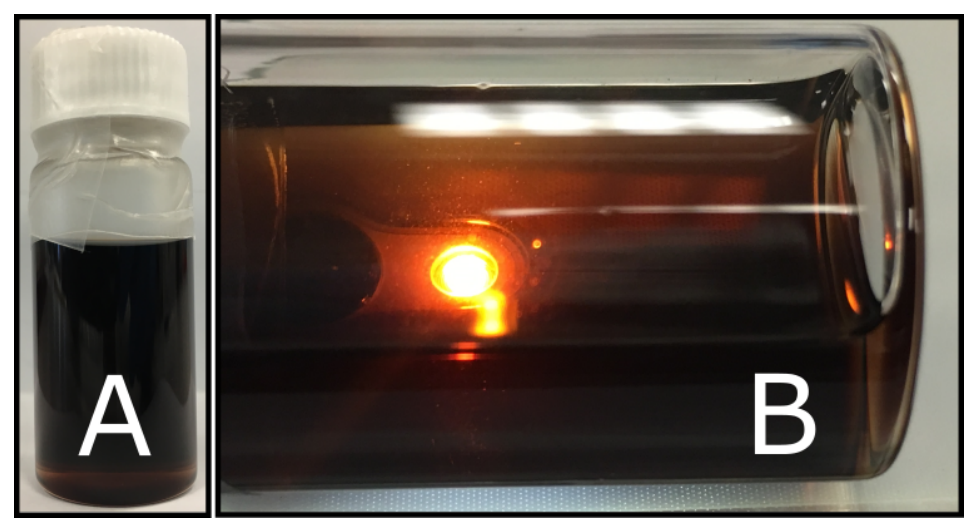

Figure 3.1: $A$, example of an $H g T e Q D$ colloidal solution. B, same as in $A$, but showing the lack of scattering through, indicating that the QDs are not aggregating in the solution.

Chemicals Used (3-Aminopropyl)trimethoxysilane (APTMS) Sigma Aldrich, $97 \%$ ), toluene (Sigma Aldrich, anhydrous, $99.8 \%$ ), hexane (Sigma Aldrich, mixture of isomers, anhydrous, > $99 \%$ ), $300 \mathrm{~nm} \mathrm{SiO}_{2}$ on Si wafers, Kapton sheets, polyethylene terephthalate sheets (PET), isopropyl alchohol (IPA), AZ1518 (Microchemicals), AZ326 (Microchemicals).

Silicon Substrates $\mathrm{Si}$ wafers with $300 \mathrm{~nm} \mathrm{SiO}_{2}$ layers were cleaved into $11 \mathrm{~mm} \times 11 \mathrm{~mm}$ squares. Patterning was achieved with an AZ1518 resist and diluted (3:1, developer:DI water) AZ326 developer. The resist was spun on at $4000 \mathrm{rpm}$ for $40 \mathrm{~s}$ and heated at $90{ }^{\circ} \mathrm{C}$ for $3 \mathrm{~min}$, development was $45 \mathrm{~s}$ in the developer and then two rinses in DI water. Post $\mathrm{Cr} / \mathrm{Au}$ deposition the lift off was achieved with acetone for silicon and Kapton and ethanol for PET.

Surface Functionalisation Initial attempts at photolithography, particularly when developing long $(\mathrm{mm})$ and thin $(\mu \mathrm{m})$ features did not work on silicon. An extreme example of photoresist (PR) delamination is shown in Figure 3.2 A. To solve this a surface functionalisation step was introduced. Once clean, the substrates were moved into a glovebox where they were 
placed into a solution of $10 \mu \mathrm{l}$ APTMS in $10 \mathrm{ml}$ toluene for $30 \mathrm{~min}$. After this they were rinsed in toluene and hexane. With the surface hydrophobically functionalised there were no further problems (Figure 3.2 B) with photolithography down to $10 \mu \mathrm{m}$.

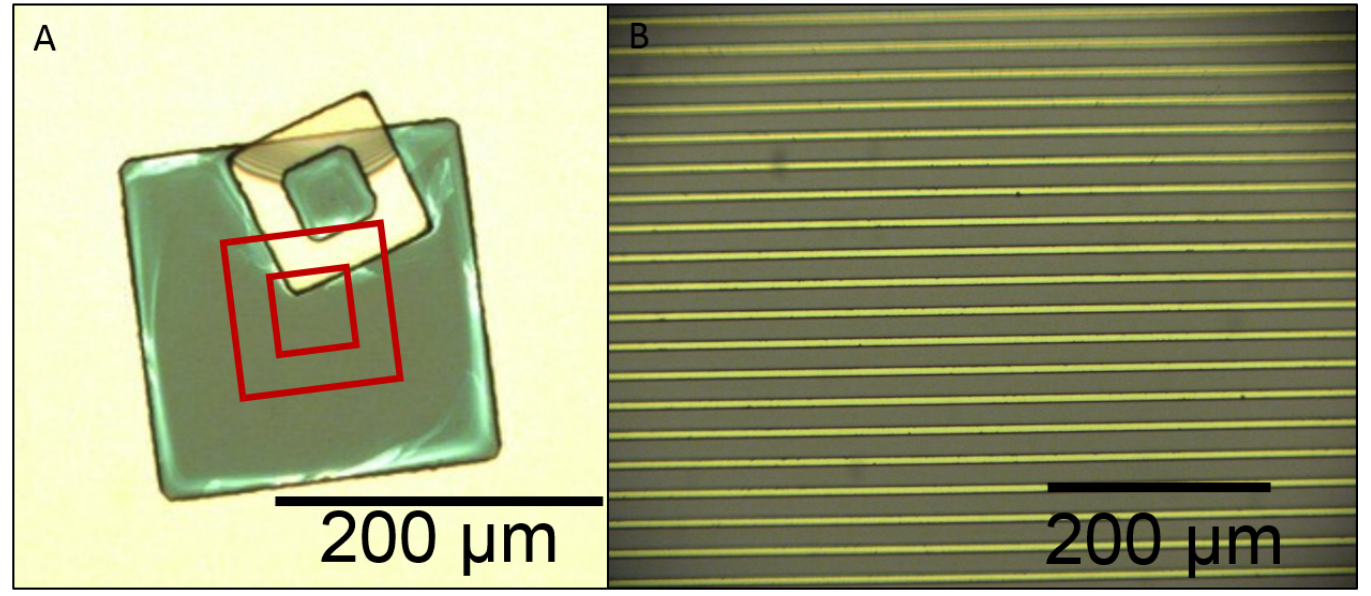

Figure 3.2: $A$, example of the photoresist $(P R)$ delaminating after the development step, the $P R$ is yellow with the exposed silicon in black. The square feature should be at the location indicated in red but it has delaminated from the silicon. B, an example of successful long and thin IDE feature development with a silane functionalised substrate.

Flexible Substrates Kapton substrates were lithographically patterned with $\mathrm{Cr} / \mathrm{Au}$ using a very similar process as for silicon, the only difference being that the substrates were heated for $10 \mathrm{~min}$ at $90{ }^{\circ} \mathrm{C}$ following the initial photoresist deposition. PET substrates were more difficult to pattern as they have a higher macro surface roughness and are not resistant to many organic solvents, in particular acetone. It was found that best results were achieved with a number of subtle changes to the photolithographic process. These were:

1. Very brief rinses in IPA followed by nitrogen drying as the only cleaning step.

2. Increased photoresist bake times as for Kapton. 
3. Lower exerted mask pressure on the mask aligner to prevent deformation.

4. Very secure mounting in the evaporator to prevent the substrate deforming under heating.

5. The use of EtOH for the lift off step. It was found that 10 min sonicating in EtOH completely removed the remaining photoresist, leaving the correct features without damaging the substrate.

With these changes it was possible to reliably fabricate $10 \mu \mathrm{m}$ trench length IDE structures. However features smaller than this were not achieved despite numerous attempts.

\subsubsection{Film Deposition Methods}

Spin Coating Various parameters were trialled and it was found that the best films were formed from QDs in chloroform solutions of a concentration in the range of $15-25 \mathrm{mg} \mathrm{ml}^{-1}$. Dynamic dispensing gave clearer films than static dispensing and a spin speed of $1000 \mathrm{rpm}$ for $40 \mathrm{~s}$ with three 20 $\mu \mathrm{l}$ drops in the first $10 \mathrm{~s}$ was the most reliable parameter set. Figure 3.3 gives a basic optical impression of film quality.

Spray Coating Spray coating was optimised for a similar concentration range and a distance of $\approx 10 \mathrm{~cm}$ between the nozzle and the device. The driving gas was nitrogen. When using chloroform solutions one must be careful to thoroughly clean the spray gun internals by flushing with $\mathrm{EtOH}$ immediately after use.

Ligand Exchange For organic ligand exchange post deposition, each layer was treated in an EDT/ $\mathrm{HCl}$ in $\mathrm{EtOH}(1: 1(3.0 \mathrm{M}): 20)$ solution to exchange the DDT capping ligands, before being rinsed in $\mathrm{EtOH}$ and dried with nitrogen. For inorganic ligand exchange each layer was treated 


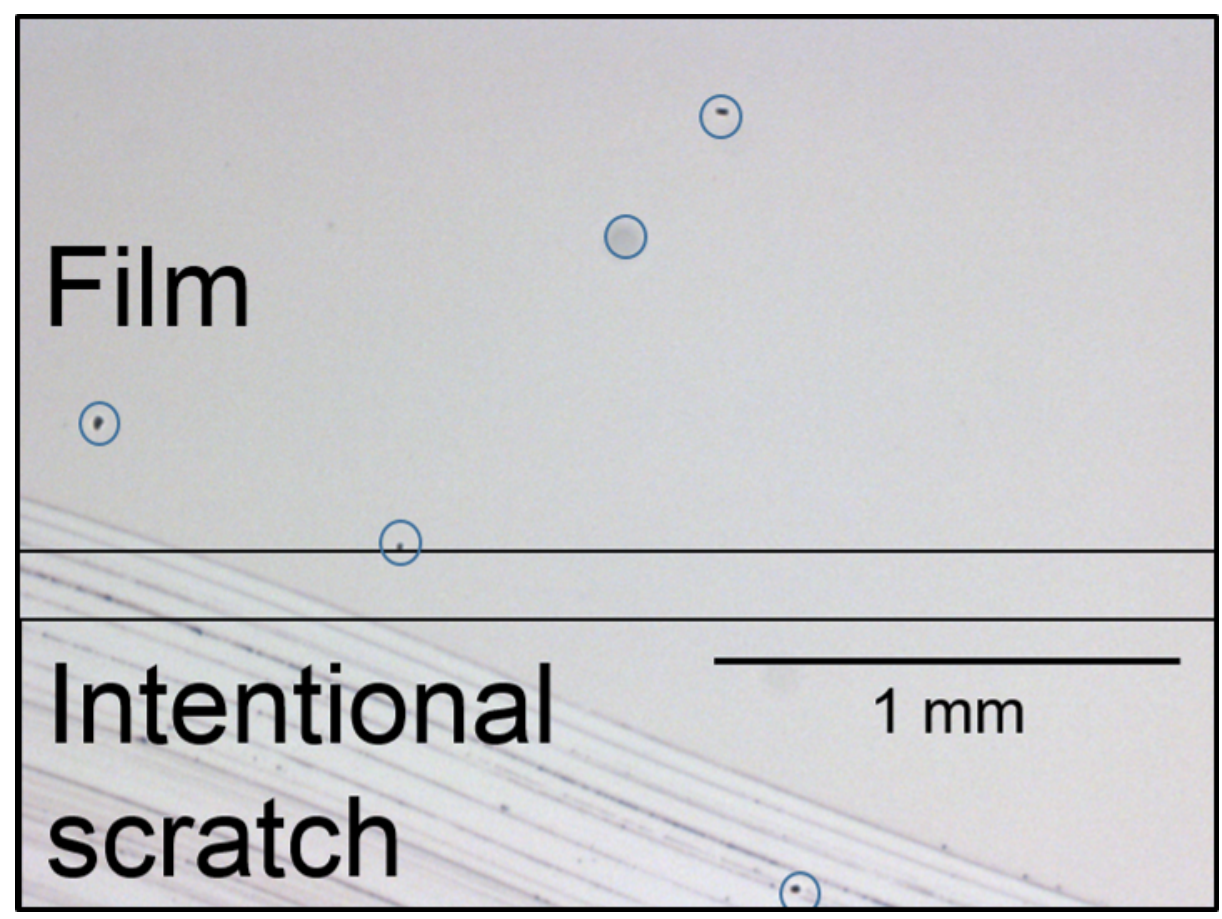

Figure 3.3: An initial understanding of whether the QDs had aggregated in the film could be given by observing the optical quality with a bottom illumination optical microscope, this was used to inform the development of the processing and device fabrication steps. The blue circles are imperfections in the microscope lens system, not film features.

in a $1.5 \mathrm{mg} \mathrm{As}_{2} \mathrm{~S}_{3}$ in $5 \mathrm{ml} \mathrm{PPA} / 5 \mathrm{ml} \mathrm{EtOH}$ solution for $30 \mathrm{~s}$ and then rinsed in EtOH before drying with nitrogen. For individual devices this process was done by hand, the rinse step was done immediately after the ligand step in order to control the length of time that the ligand solution could interact with the QD film. Following rinsing the device was placed at an angle and then dried with a nitrogen gun, at the drying stage the success of the deposition could be decided. Where unsuccessful the nitrogen stream would cause the films to flake off the substrate. Due to the highly toxic nature of the materials being used this process required careful planning and a designated area. For devices in stencil mounts the exchange process was simplified by placing the entire mount in the required solution, then carefully drying the mount to ensure no 
liquids remained in contact with the substrates. One further practical consideration was the use of bespoke containers matched to the sample mount size so that the smallest quantities of ligand exchange solution could be used, both a financial and safety consideration.

\subsubsection{Characterisation}

Electrical Measurements Electrical measurements were conducted on a Keithley SCS-4200 parameter analyser (PA) with co-axial cabling. The device was mounted in the optical chamber of a Janis VNF-100 on a temperature controlled mount and electrical connections ran from the mount to the PA. The mount was held at $293 \mathrm{~K}$ (heated to just above ambient temperature) for all measurements. A Signal Recovery 7265 digital lock in was used for the noise measurements and these were conducted using a constant $10 \mathrm{~V}$ source connected through the device to the current input of the lock in. The amplifier was then operated in the self-reference mode $[107,110]$. As the system was not perfectly isolated from power line noise the chosen measurement frequencies were not multiples of $50 \mathrm{~Hz}$, i.e. $101 \mathrm{~Hz}$ not $100 \mathrm{~Hz}$. High speed real-time measurements were taken using a Tektronix TBS1000B oscilloscope.

Optical Path The optical path remained as described in Chapter 2, the aperture of an Omega BB-4A blackbody, the longpass filter, $12 \mathrm{~cm}$ of air shielded from ambient light, an optical chopper and the two sapphire windows of the cryostat. The $3 \mathrm{~K}$ LP and $4 \mathrm{~K}$ LP filters were purchased from Spectrogon and the $1.65 \mathrm{~K}$ LP and $2.4 \mathrm{~K}$ LP filters were purchased from Edmund Optics. The incident power on the device was calculated by integrating the spectral Planck blackbody power radiance equation for each optical wavelength (per nm) over the known transmission of the path. The solid angle of the source/filter path as seen by the device was calculated by calibrating with a Thorlabs PM1000D power meter with S401C detector. Knowing the radiance at the device distance, the detected thermal 
power and the known area of the detector allows the effective solid angle of the source/filter to be calculated. This methodology is covered in detail in Chapter 2.

\begin{abstract}
Absorbance Attenuated Total Reflectance (ATR) and path absorbance measurements were both taken on a Bruker Tensor T27 FTIR with the ATR and disc attachments respectively. ATR measurements were taken with the NC film dried onto the crystal and transmission measurements used an NC film cast onto $1 \mathrm{~mm}$ thick $\mathrm{CaF}_{2}$ substrates purchased from Eskma Optics. In both cases these films were optically clear and not a powder.
\end{abstract}




\subsection{Results}

\subsubsection{QD Characterisation}

The synthesis method above produces tetrapodal HgTe QDs that show tunable bandgaps throughout the MIR by changing the cook temperature. This is shown by the absorbance of the colloids when dried onto the crystal of an FTIR ATR attachment in Figure 3.4 and by the size increase of the QDs in TEM images (Figure 3.5). The use of ODT ligands for im-

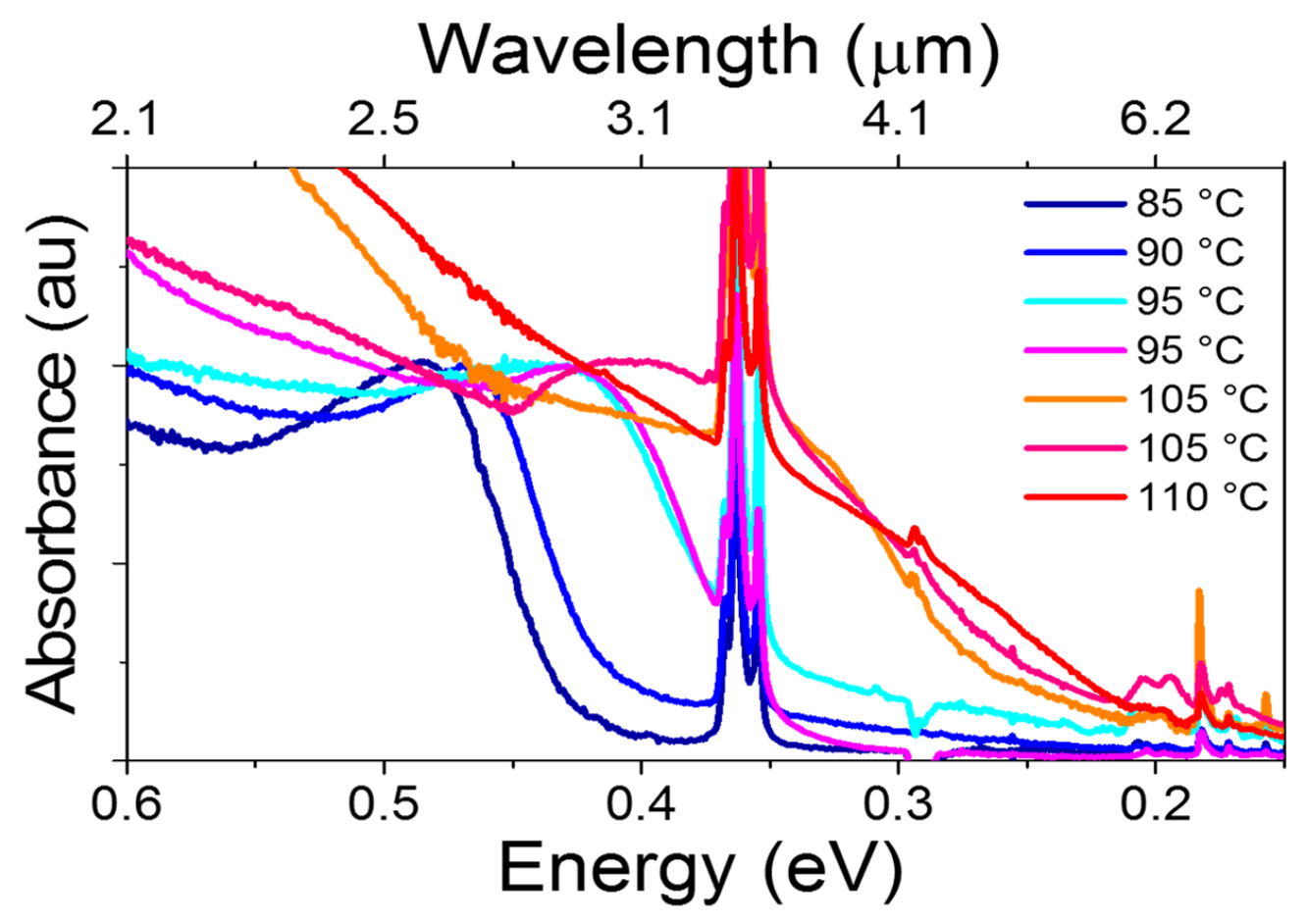

Figure 3.4: The bandgap of the QD films can be tuned through the cook temperature of the synthesis. Higher cooking temperature results in lower energy bandgaps. These results are for a variety of QD preparations synthesised when developing the process.

proved inter-QD separation is shown in Figure 3.6. This is useful when preparing QDs for imaging, however the experimental difficulty of using a ligand that is solid at room temperature means this should only be done 


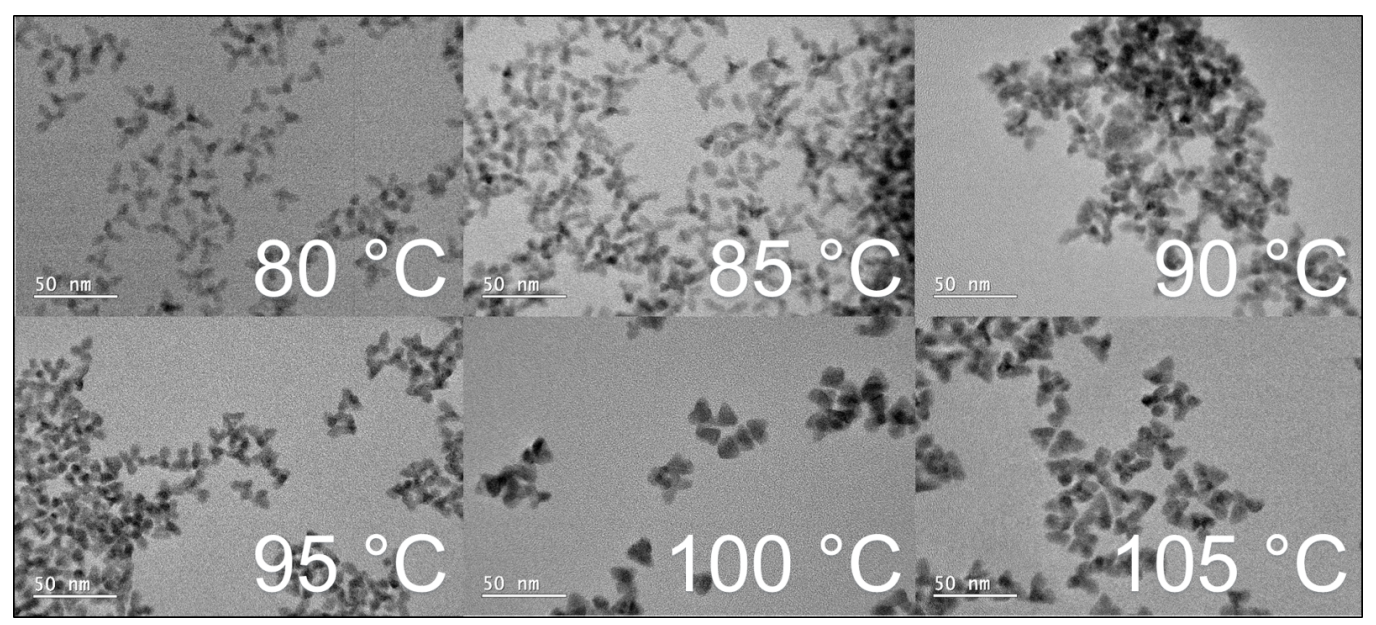

Figure 3.5: The bandgap of the $Q D$ films can be tuned through the cook temperature of the synthesis. Higher temperature results in larger QDs. The temperatures shown relate to Figure 3.4.

when normal ligands are not facilitating good imaging. The HgTe crys-

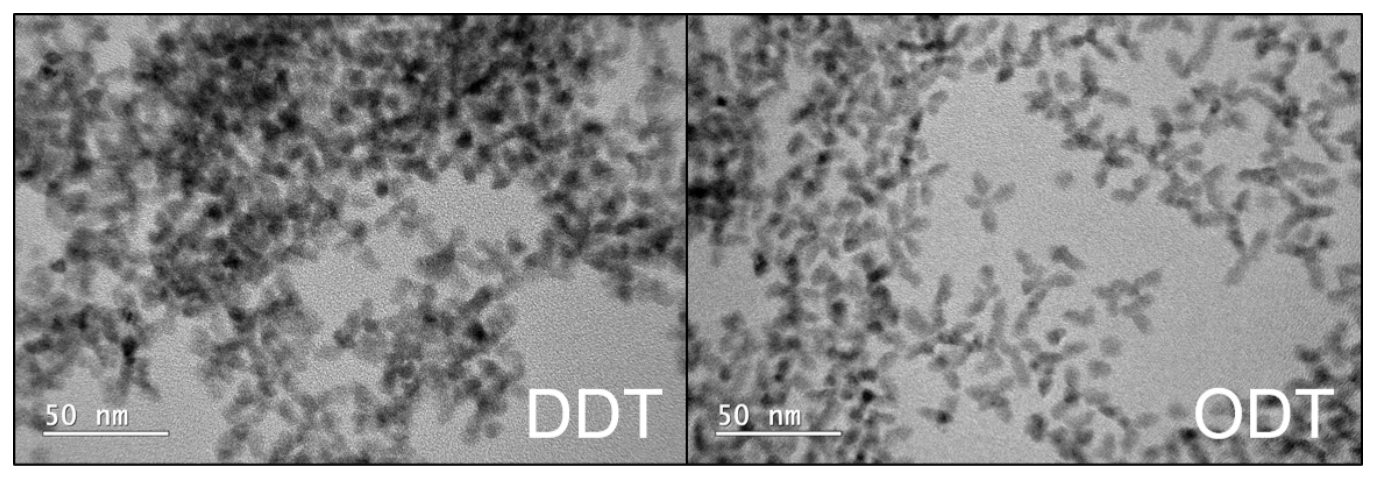

Figure 3.6: Same QD sample but capped with the ligand indicated following synthesis, the ODT capped QDs are visibly more disperse as the longer ODT ligands cause a greater physical separation between QDs in the solution and on the TEM grids.

tal structure is confirmed by XRD, TEM diffraction and TEM crystal plane spacing, shown in Figure 3.7. These results are not novel but importantly confirm that the QDs are definitely $\mathrm{HgTe}$ as they match the data given in PDF 00-032-0665. 


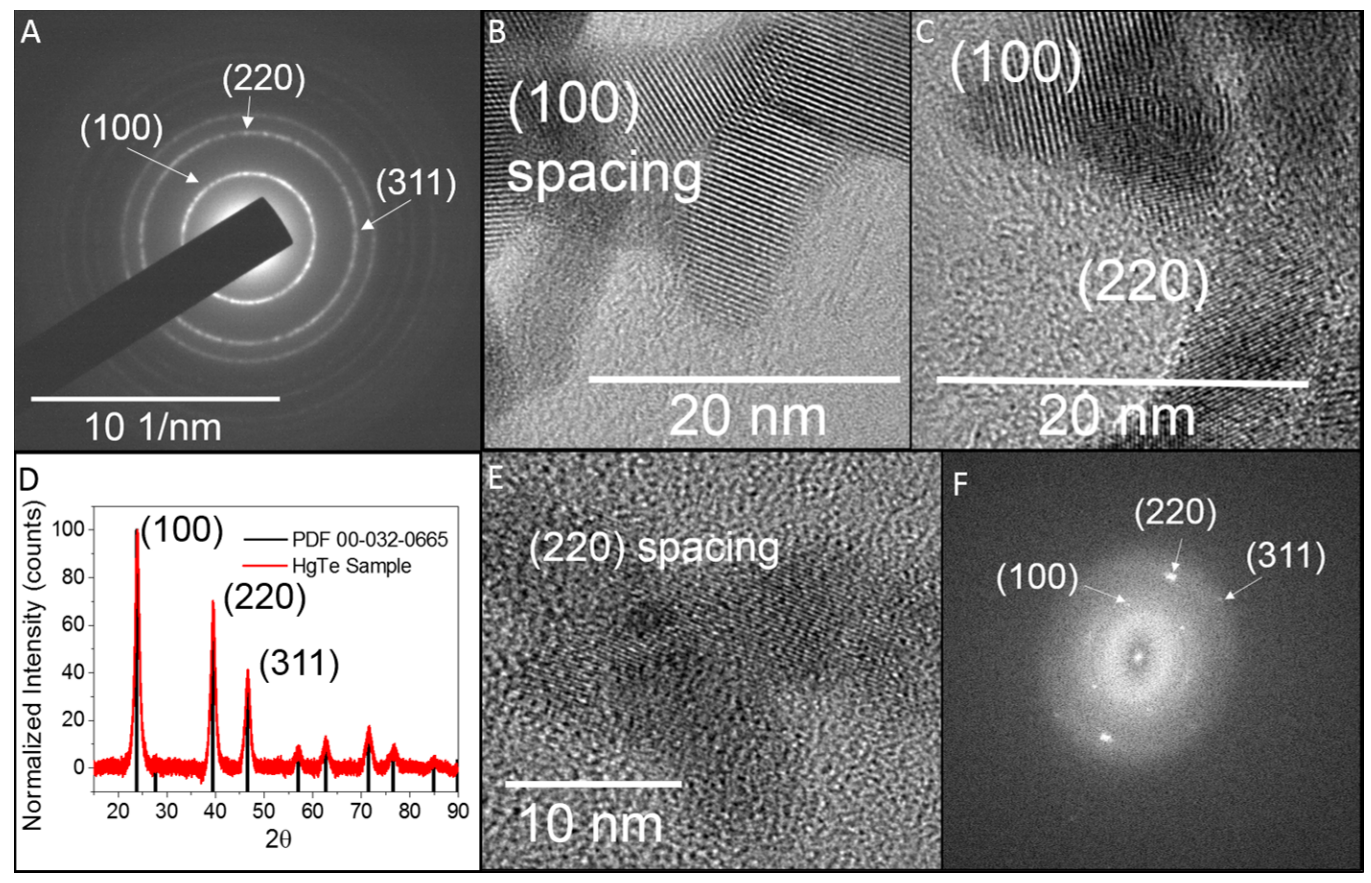

Figure 3.7: $A, S A E D$ of a large number of $Q D$ s, as the ensemble is polycrystalline rings are observed instead of points. The rings match the lattice spacing for the planes indicated, and relative intensities match the XRD. B, TEM showing very clear 100 plane spacing. C, TEM showing 100 and 220 lattice spacing (indicated). D, XRD of film with the reference file and the three major planes indicated. E, HRTEM of single crystalline area, 220 is dominant. F, single crystal diffraction of same area, points visible as monocrystalline sample, brightest point corresponds to 220, as expected from $E$.

\subsubsection{Organic Ligands on Silicon Substrates}

The DDT ligands that are initially passivating the surface are long chain aliphatic molecules. These account for the $\mathrm{C}-\mathrm{H}$ stretch regularly seen in this work around $3000 \mathrm{~cm}^{-1}$ in the absorbance plots (Figure 3.4). Electronically these ligands serve to insulate the QDs from each other and as such it would be expected that there is a very high resistance in the films. As an example it can be seen that three spin coated layers of DDT capped QDs on an IDE with $20 \mu \mathrm{m}$ length electrodes with a total width of $78 \mathrm{~mm}$, gives a current of $4 \mathrm{nA}$ at $10 \mathrm{~V}$ bias (Figure 3.8). If PC behaviour is to be easily observed then the film resistance will have to be reduced. If this was 


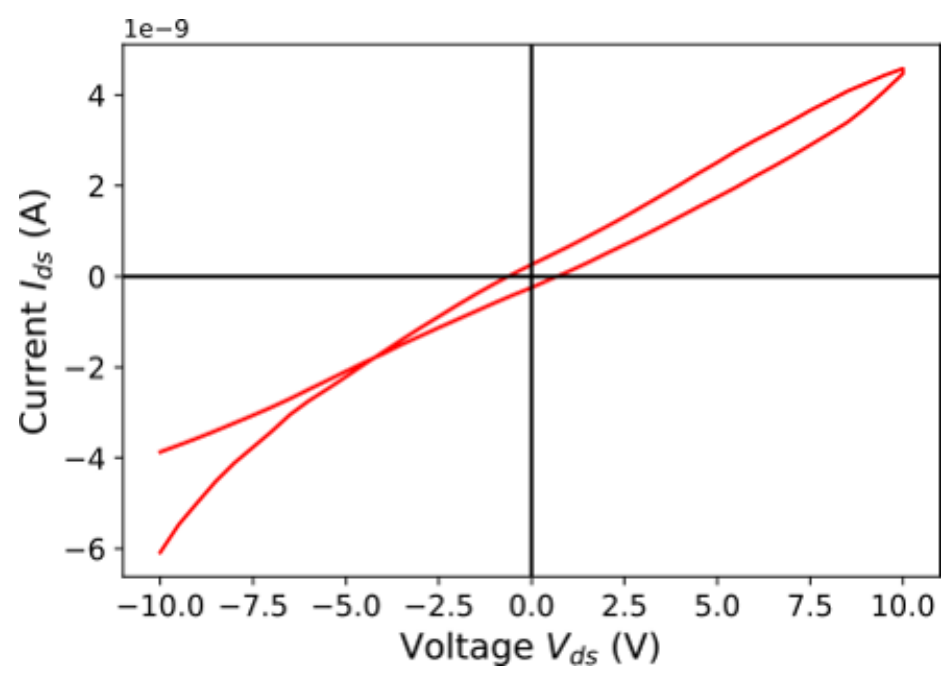

Figure 3.8: IV plot for a three layer film of DDT capped QDs, spin coated onto a functionalised silicon device without any post deposition ligand treatment. Without ligand treatment the measured current was in the $\mathrm{nA}$ range. Features such as the hysteresis are discussed in Chapter 4.

the only objective then the simplest solution to this would be to sinter the films. This would cause agglomeration and the film conductance would go up as conduction would now be through the bulk aggregated material. However, this would detrimentally affect the optical band edge and remove the band edge selection achieved through size tuning. In order to increase the conduction but maintain the band edge the most obvious solution is to reduce the inter-particle separation by replacing the DDT ligands with a shorter molecule that will passivate the QD surface equally well. Currently the most popular solution is to use EDT in EtOH in a post deposition ligand exchange [54]. Conducting the exchange on the already formed film will never result in a complete exchange as some DDT will always be geometrically trapped. This method would also be expected to generate visible cracking as the film volume reduces, which is not ideal from a conduction perspective. However it is simple to achieve, easy to reproduce and is easily testable with a multimeter. Two example films are shown in Figure 3.9 A. Casting the film, measuring in air and then con- 
ducting the EDT exchange still results in a clear conductivity increase. It can be seen from Table 3.1 that this exchange process works well and consistently reduces the measured resistance by 4 or 5 orders of magnitude (relative to a test device in line one). The actual measured mean height of the $\mathrm{Cr} / \mathrm{Au}$ features in these devices was $3 / 30 \mathrm{~nm}$ above the substrate. The height of three layers of EDT treated film is $30 \mathrm{~nm}$ (see Chapter 4) therefore conductivity was calculated with a height of $10 \mathrm{~nm}$ per layer. This explains why the increasing height of four layers gave little benefit and was the reason that three spin coated layers was the default device fabrication method. Figure $3.9 \mathrm{~B}$ shows that the $\mathrm{C}-\mathrm{H}$ stretch of the films is heavily reduced by the replacement of a 12 carbon chain molecule (DDT) with a two carbon chain molecule (EDT).
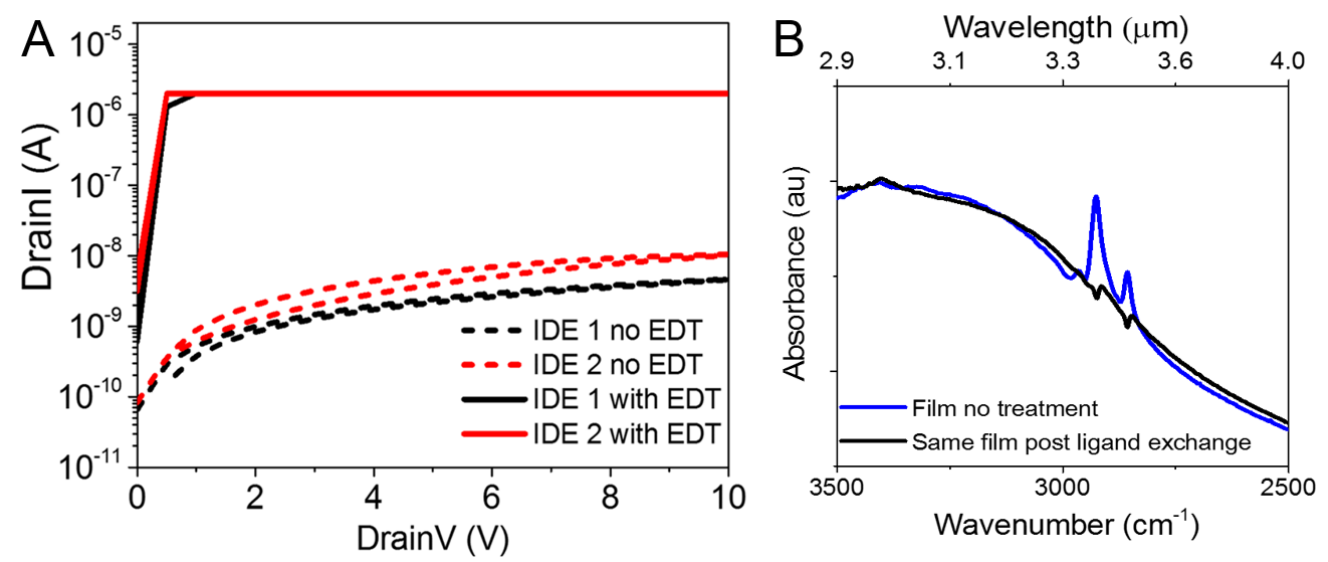

Figure 3.9: $A$, logI-V plot for two films on $20 \mu \mathrm{m}$ silicon IDEs, the PA had a compliance of $2.0 \mu \mathrm{A}$ hence the EDT treated samples flat line. Plotted as such to show the three decade increase in measured current, for the same film, caused by the EDT ligand treatment. B, the absorbance change due to EDT treatment, note the reduction in the $\mathrm{C}-\mathrm{H}$ stretch. This shows that the ligand exchange removes $\mathrm{C}-\mathrm{H}$ bonds from the material, this could be due to a complete removal of all ligands in the film and thus the aggregation of the QDs. The evidence that this is not the case and that the QDs remain separated is the preservation of the bandgap in the film, which is not the same as the bulk bandgap. This preservation of bandgap can also be seen here. 


\begin{tabular}{lllllll} 
Layers & $\begin{array}{l}\text { IDE Spac- } \\
\text { ing }(\mu \mathrm{m})\end{array}$ & $\begin{array}{l}\text { Channel } \\
\text { length }(\mathrm{m})\end{array}$ & $\begin{array}{l}\text { Channel } \\
\text { Width }(\mathrm{m})\end{array}$ & Area $\left(\mathrm{m}^{2}\right)$ & Measured $\mathbf{R}(\Omega)$ & $\rho(\Omega \mathrm{m})$ \\
\hline 3 & 20.0 & $2 \times 10^{-5}$ & 0.124 & $3.72 \times 10^{-9}$ & $2.0 \times 10^{9}$ & $3.7 \times 10^{5 *}$ \\
\hline 2 & 60.0 & $6 \times 10^{-5}$ & $5.6 \times 10^{-2}$ & $1.12 \times 10^{-9}$ & $9.1 \times 10^{5}$ & 17 \\
\hline 3 & 60.0 & $6 \times 10^{-5}$ & $5.6 \times 10^{-2}$ & $1.68 \times 10^{-9}$ & $5.0 \times 10^{5}$ & 14 \\
\hline 4 & 60.0 & $6 \times 10^{-5}$ & $5.6 \times 10^{-2}$ & Over feature height & $4.8 \times 10^{5}$ & \\
\hline 2 & 40.0 & $4 \times 10^{-5}$ & $7.8 \times 10^{-2}$ & $1.56 \times 10^{-9}$ & $4.1 \times 10^{5}$ & 16 \\
\hline 3 & 40.0 & $4 \times 10^{-5}$ & $7.8 \times 10^{-2}$ & $2.34 \times 10^{-9}$ & $1.0 \times 10^{5}$ & 5.9 \\
\hline 4 & 40.0 & $4 \times 10^{-5}$ & $7.8 \times 10^{-2}$ & Over feature height & $8.9 \times 10^{4}$ & \\
\hline 2 & 20.0 & $2 \times 10^{-5}$ & 0.124 & $2.48 \times 10^{-9}$ & $1.0 \times 10^{5}$ & 12 \\
\hline 3 & 20.0 & $2 \times 10^{-5}$ & 0.124 & $3.72 \times 10^{-9}$ & $1.0 \times 10^{4}$ & 1.9 \\
\hline 4 & 20.0 & $2 \times 10^{-5}$ & 0.124 & Over feature height & $9.2 \times 10^{3}$ & \\
\hline
\end{tabular}

Table 3.1: Table showing the resistivity of initial film measurements, ${ }^{*}$ this control sample had no ligand exchange. Over feature height means that the height of the film is greater than the height of the electrodes therefore resistance cannot be accurately converted into resistivity. The resistance value is only given to 2 s.f. as all these devices, when measured in normal lighting conditions, have a fluctuating resistance, hence the resistivity value is also only given to 2 s.f., this is accuracy suffices here as it is merely required to show a trend in behaviour.

Temperature Dependence The dark temperature dependent conductivity of a typical EDT treated film is shown in Figure 3.10, it shows a clear peak around $200 \mathrm{~K}$. This is in agreement with other published temperature dependence of EDT treated films [54,99]. Simple temperature dependence measurements are not novel, Chapter 4 goes into more detail about how temperature affects the conduction in these films with reference to a novel behaviour. As a brief discussion of these results it is curious that there is a peak of conductivity and then a drop with increasing temperature. The conductivity in these films is dependent on the hopping mechanism and the carrier concentration. The carrier concentration will increase with temperature, as should the hopping rate (NNH). Therefore properly explaining the drop in conductivity is a clear area for future work, building on the work in Chapters 4 and 5 it appears that trap population in these air exposed films increases with temperature, and may be responsible for the observed reduction. 


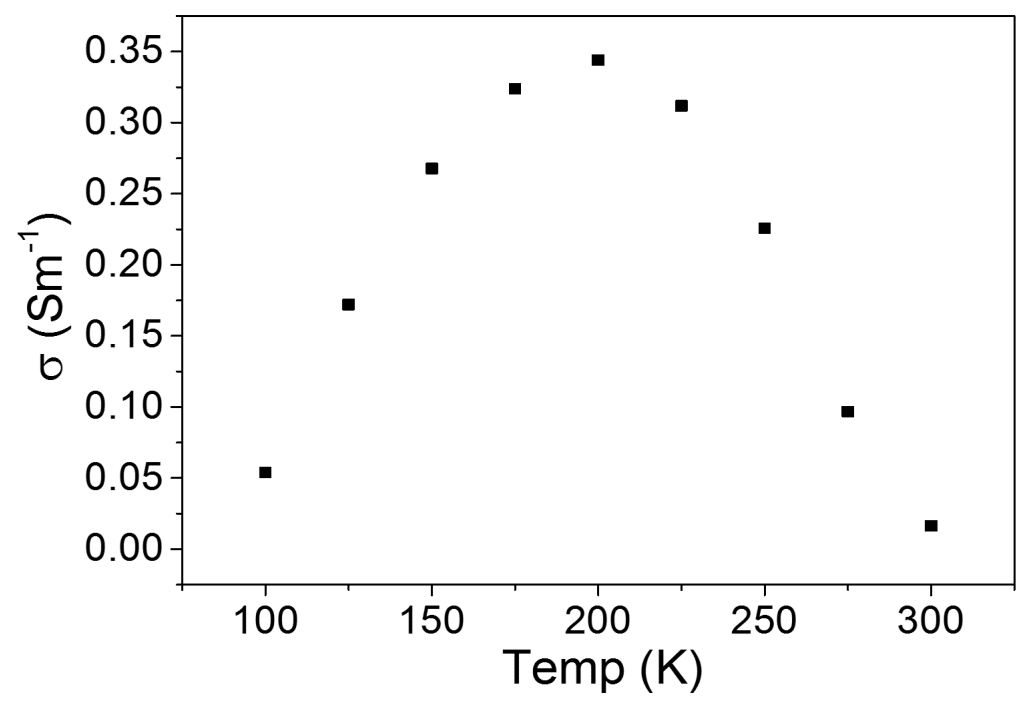

Figure 3.10: Dark conductivity with temperature plot, the conductivity values were calculated by taking low bias measurements and calculating the gradient through the origin, i.e. it is a measure of the conductivity in the zero bias condition, not field driven conductivity. 


\subsubsection{MIR Photodetection with Silicon Substrates}

At the time this work was completed MIR detection with cooled films on silicon had already been achieved $[43,99]$ however reproducing this represented a significant and necessary milestone in developing the materials, devices and testing procedures from the ground up, as was done in this work. It can be seen in Figure 3.11 that these early, cooled devices did function, having a full scale responsivity that is $>0.1 \mathrm{~A} \mathrm{~W}^{-1}$. However as the time response is poor the $1 \mathrm{~Hz}$ responsivity is only a small proportion of the full change. There is also a current drift that is a large proportion of on/off signal. As such the performances shown briefly here were an encouraging first step but in no way represented progression in the wider literature. They also give context for the results presented at the end of this Chapter, and most importantly acted as a proof of concept for the measurement set up.

With incremental improvements in device fabrication it was possible to fabricate EDT based detectors that demonstrated MIR detection reliably at temperatures up to $240 \mathrm{~K}$. As expected these devices had a maximum responsivity that corresponded with the maximum conductivity, at $200 \mathrm{~K}$. This is shown for a $773 \mathrm{~K} \mathrm{BB}$ in Figure 3.12 A and for a $773 \mathrm{~K}$ BB with 3K LP filter in place in Figure 3.12 B. The latter is an important measurement as due to the longpass filter we know that any electrical response is caused entirely by photons that have an energy lower than $413 \mathrm{meV}$.

Along with the improved responsivity, the time response of these optimised films was also considerably increased. It can be seen from Figure 3.13 A that there are now clear fast and slow components to both the leading and trailing edge response to an optical signal. However Figure 3.13 $B$ shows that the fast component is still only a small fraction of the rising edge even when using moderately slow signals. The device still shows the full on signal because it does not clear all generated charges in one time 


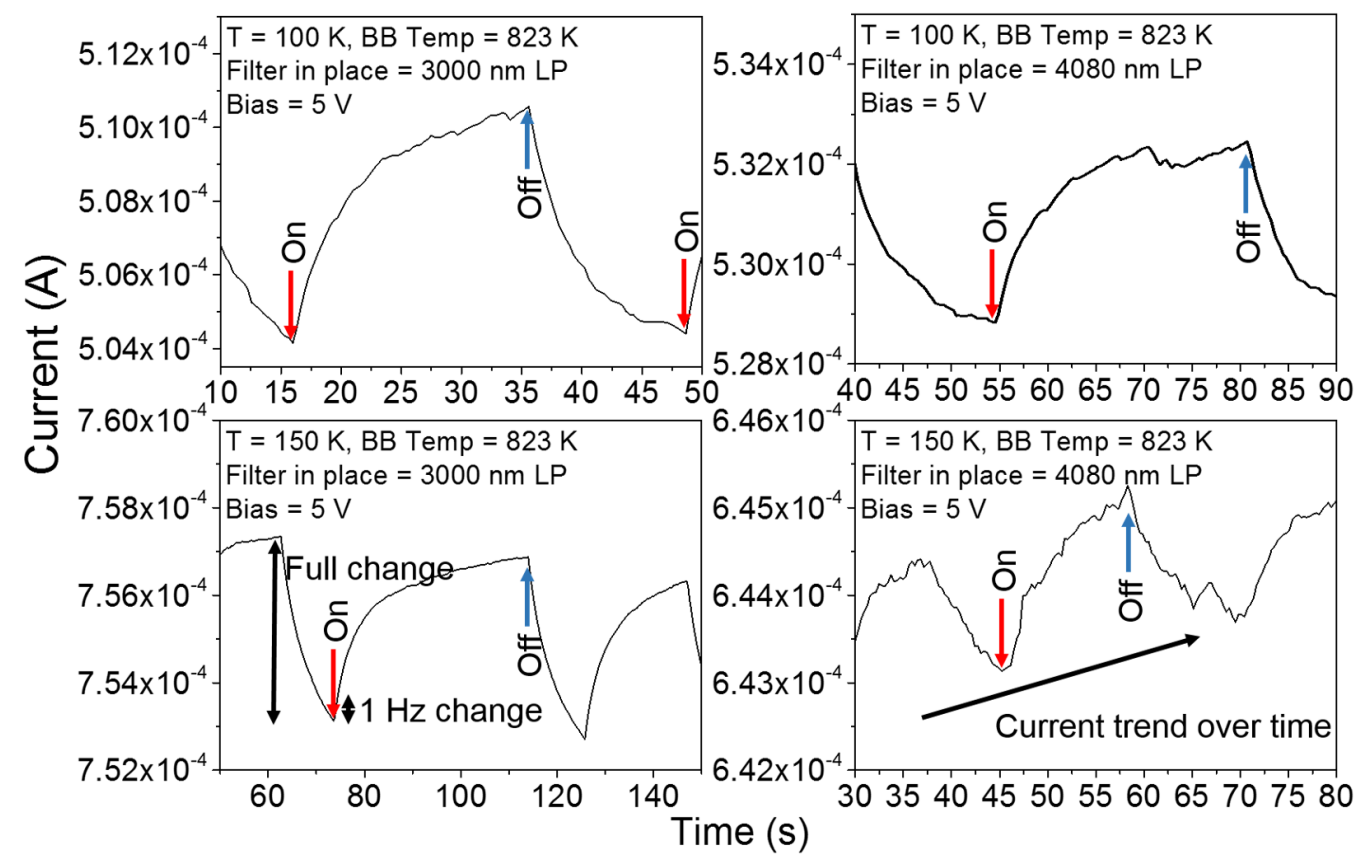

Figure 3.11: A variety of current time plots with the parameters shown in each plot. In all these devices and conditions the $1 \mathrm{~Hz}$ response is only a small proportion of the total current change due to exposure, i.e. the photoresponse is slow. Some devices also showed a current trend over time when held at a specific temperature. The filter refers to the optical filters initially discussed in Chapter 2.

period. The boxed area in the figures draws the eye to the true response to the pulsed optical signal, i.e. what current change is observed in $(1 / f)$ s. Here, even the the fast component of the rise time at $20 \mathrm{~Hz}$ is only $\approx 10$ $\%$ of the full response shown in Figure $3.13 \mathrm{~A}$. In short, the rise time of these devices is too slow to be competitive in imaging metrics. From here the next performance goal of these detectors was to improve both the time response and the maximum temperature of operation.

\subsubsection{Post Deposition Inorganic Ligand Exchange}

Lhuillier et al. demonstrated that a post deposition ligand exchange with $\mathrm{As}_{2} \mathrm{~S}_{3}$ in air-free conditions resulted in higher temperature photoresponse 


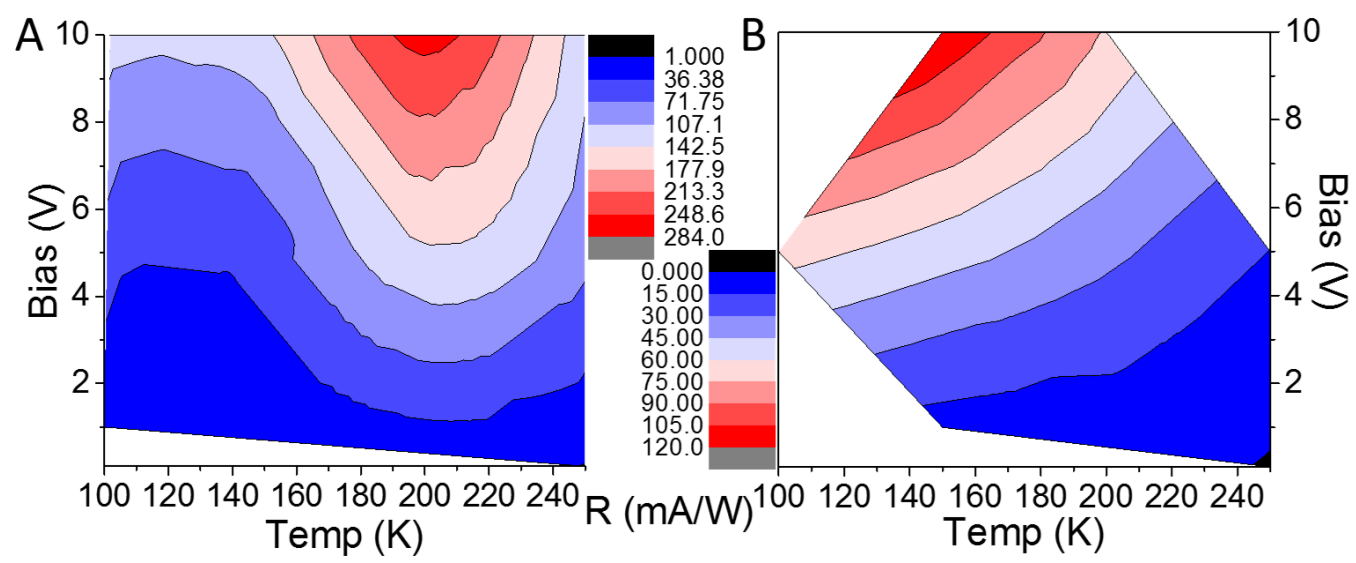

Figure 3.12: $A$, responsivity contour plot for varying temperature and bias. The optical signal is an unfiltered $773 \mathrm{~K} \mathrm{BB}$. Here the peak of responsivity occurs at $\approx 200 \mathrm{~K}$ as would be predicted from Figure 3.10. B, in this case the optical signal is a $3 K$ LP filtered $773 \mathrm{~K} \mathrm{BB}$. It is not known why this device showed a different temperature response to that seen in $A$.
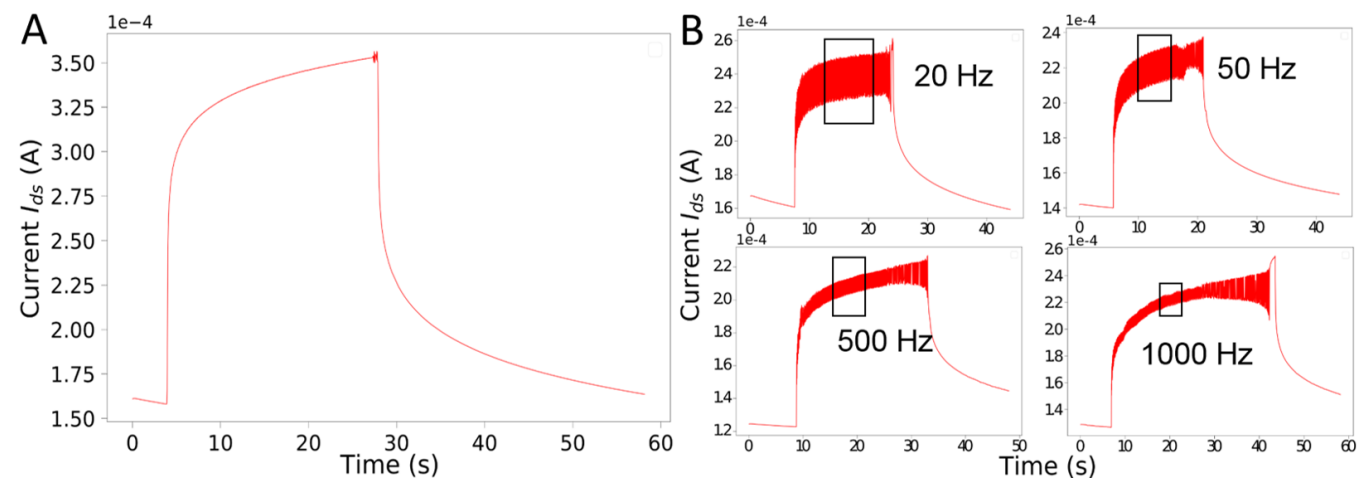

Figure 3.13: A, current-time plot showing photoresponse to a $670 \mathrm{~nm} L D$ with single on pulse, at $200 \mathrm{~K}$. B, current-time plots for a $670 \mathrm{~nm}$ LD being turned on with an optical chopper at the indicated frequency. The rectangles are a guide for the eye, by looking at the areas highlighted it can be seen qualitatively that as the modulation frequency of the incident light increases the on/off current response reduces, all measured at $200 \mathrm{~K}$.

[99]. As previously stated, the aim here was to develop a low cost room temperature detector, so the $\mathrm{As}_{2} \mathrm{~S}_{3}$ exchange was adapted for use in air. It was found that lower concentrations of $\mathrm{As}_{2} \mathrm{~S}_{3}$ in the propylamine/ethanol solution gave better results in air. At this point in the work the deposition 
method was changed from spin coating to spray deposition as spray coating allows easy 2-D patterning of the film. The largest hurdle to overcome in the post deposition inorganic exchange process was preventing delamination of the films. If the ligand exchange solution was too concentrated, specifically $>5 \mathrm{mg} \mathrm{As} \mathrm{S}_{3}$ per $5 \mathrm{ml}$ propylamine then the film delaminates and is destroyed even by gentle pressure from the nitrogen gun. An example of this seen through an optical microscope is at Figure 3.14. The lower bottom right area of the film has cleanly removed itself from the substrate, indeed there is almost no indication that there was previously a well adhered film. Figure 3.14 additionally demonstrates the level of coverage that can be achieved with just one spray coated layer. This would not be

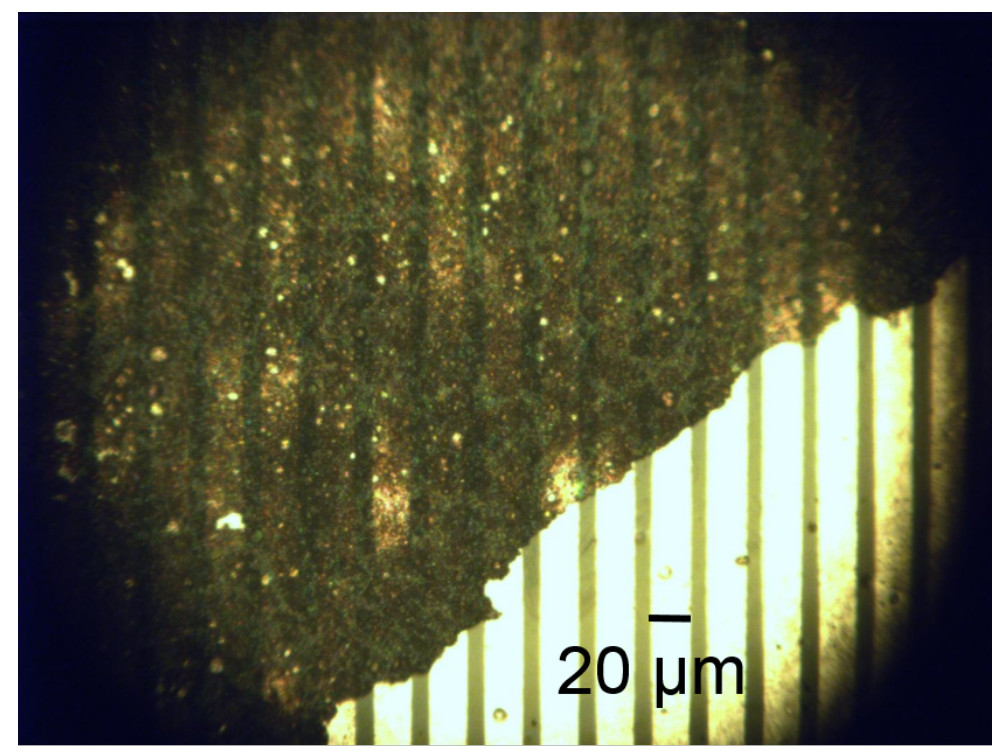

Figure 3.14: Optical microscope image of a partially removed film, following the $A s_{2} S_{3}$ ligand exchange the film delaminates from the substrate, the device pictured here shows little electrical response even though it appears that the film is still in place across $50 \%$ of the IDEs.

such a large fabrication problem if the remaining film was still well integrated with the Au IDEs, however the electrical behaviour of a partially delaminated device was very similar to a clean substrate, i.e. any electrical connectivity within the trenches had been removed. Although not a 
particularly exciting avenue for future research, a detailed study into the behaviour of these materials would be very interesting as it may lead to vastly improved ligand replacement methodologies. Initial attempts to solve this problem included (1) no exchange on the initial DDT capped layer that was set with an ethanol rinse and then continuing with the inorganic exchange for the remaining layers. This resulted in a marginally less insulating film than a completely non-exchanged film. (2) the use of EDT in the first layer, which resulted in delamination after the second layer was treated with the inorganic ligands.

Films treated with a low ligand concentration that did not cause delamination allowed the inorganically capped films on $\mathrm{SiO}_{2}$ to be characterised. Unlike organically treated films there is no hysteresis in the freshly prepared devices. The devices were initially tested with a protocol designed to ensure that the device responds as expected when cooled. This is done at $100 \mathrm{~K}$ so that there is a difference between the dark condition, where the sensor is exposed to the equivalent of a $100 \mathrm{~K} \mathrm{BB}$, and the room temperature background condition which is the equivalent of a $290 \mathrm{~K} \mathrm{BB}$. Figure 3.15 A shows that the measured current increases with incident radiation as expected. With even background room temperature being detectable above the dark current. MIR only incident radiation was also detectable, as tested with the $3 \mathrm{~K}$ longpass filter in place. These results for a cooled device were expected but represented another milestone. Figure 3.15 B shows the measured current with temperature from $200 \mathrm{~K}$ to $300 \mathrm{~K}$. Unlike films treated with organic ligands these inorganic films have a higher conductivity at $300 \mathrm{~K}$ than at 100 or $200 \mathrm{~K}$, as such it is possible that they can also show photoresponse at room temperature. The inset shows initial photoresponse measurements at a variety of temperatures. These values are notable as they are from an NC film, at room temperature and processed in air and as such they offered a promising base from which to continue to design a microbolometer competitive sensor. Response to vis- 

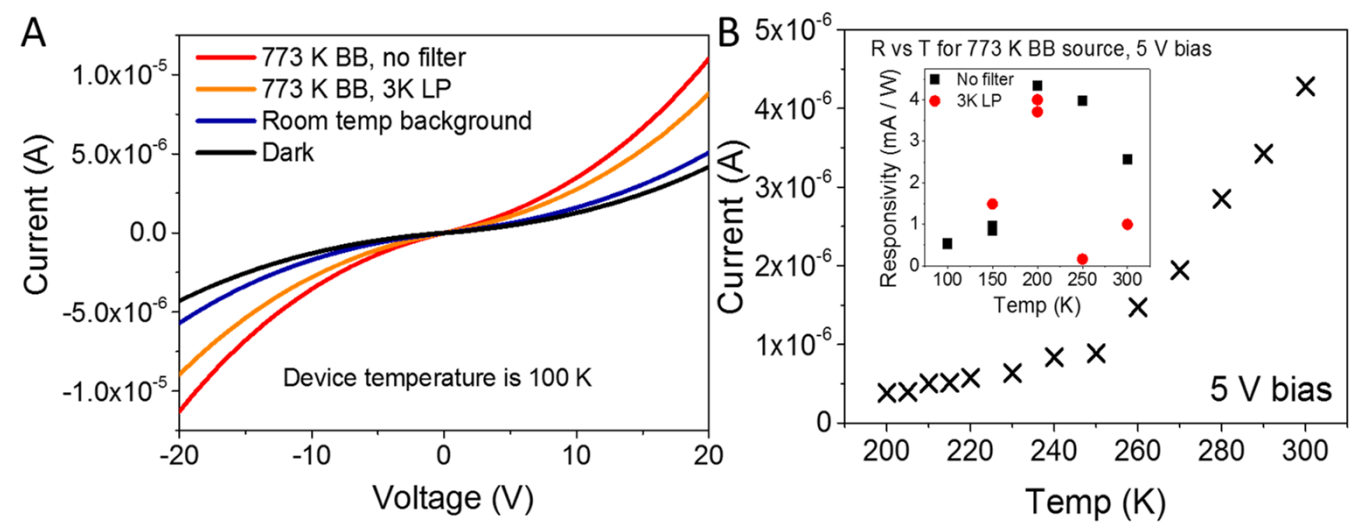

Figure 3.15: $A, I V$ plots for a $2.9 \mu \mathrm{m}$ bandgap film at $100 \mathrm{~K}$. The device is sensitive to the changes in incident radiation for the 4 levels shown. $B$, the dark current changing with temperature above $100 \mathrm{~K}$, unlike QD films treated with EDT (can be seen in Figure 3.10) the current increases continually with temperature up to $300 \mathrm{~K}$. The inset shows the responsivity with temperature for this initial device.

ible light for these devices was wavelength dependent as shown in Figure 3.16. There is a visible imbalance between the fast and slow components that is affected by the wavelength. One simple explanation for this is that higher energy photons create more hot carriers and these can be trapped in a wider (energetically) range of traps, therefore increasing the slow component. This is a clear area for further research and could be accomplished easily and quickly with transient absorption spectroscopy.

\subsubsection{Kapton Substrates}

Having shown that the use of inorganic ligands in air facilitated MIR detection at room temperature on silicon substrates, it was necessary to find a more robust and cheaper substrate material to remove the cost and fragility of silicon wafers from the device. More robust substrates allow a greater pressure to be exerted on the surface as you cannot damage the dielectric layer and therefore give a larger error allowance when setting up a stencil mask for spray coating, as well as the clear advantage of lower cost. An obvious first choice was to use Kapton (a polyimide film) 

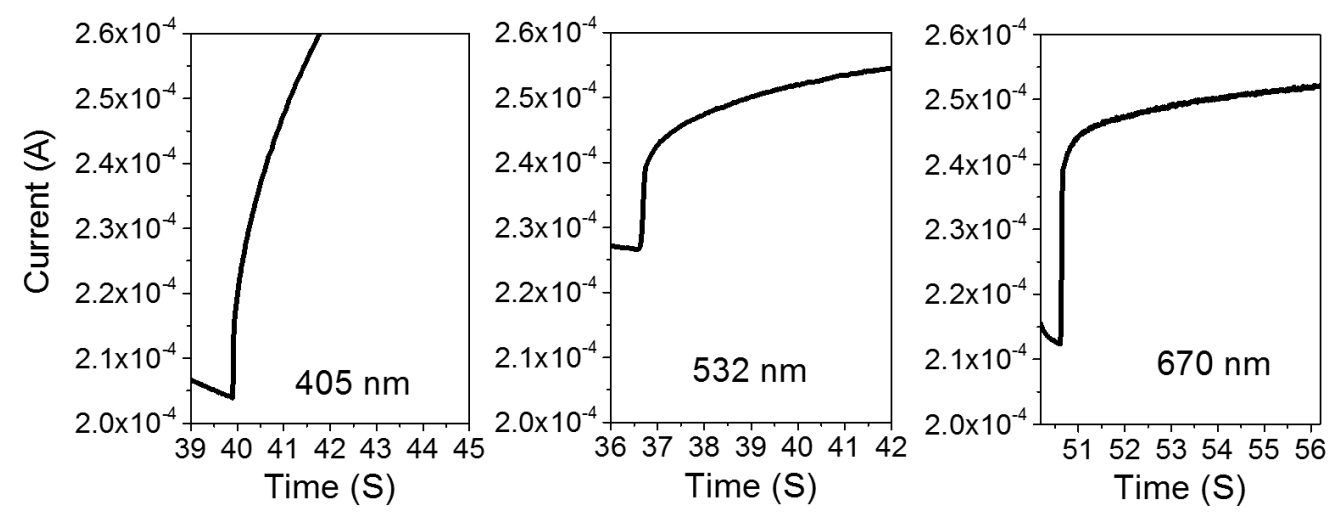

Figure 3.16: Current-time plots for the same film excited with different wavelengths of visible light. Each laser diode has the same power $(4.5 \mathrm{~mW})$ incident on the IDE.

as this has a strong track record in flexible transistor structures [135].

One potential limiting factor was the ability to fabricate precise and regular IDE structures on these substrates. Kapton works very well with simple 1-step photolithography and reliably produced IDE structures with 5 and $10 \mu \mathrm{m}$ IDEs. However Kapton substrates were found to be completely unsuitable as inorganically capped films would not adhere, leading to complete delamination in all instances, similar to that shown in Figure 3.14. The exact cause of the delamination here is unknown, however as the Kapton works well with the EDT in EtOH exchange one can surmise that the cause is the interaction at the interface between either the PPA or $\mathrm{As}_{2} \mathrm{~S}_{3}$. As the $\mathrm{EtOH}$ based exchange works well on various surfaces it is evident that the QDs when capped with DDT or EDT, are more strongly bound to the surface than they are stable in a polar solution. Additionally, as the Kapton has a relatively low wetting angle there must be some species at the surface that has weak dipole behaviour, which allows water to form a weak hydrogen bond at the surface. This same surface dipole behaviour would be energetically unfavourable to an $\mathrm{As}_{2} \mathrm{~S}_{3}$ capped QD compared to the surface of another $\mathrm{As}_{2} \mathrm{~S}_{3}$ capped QD. Thus the film of QDs sticks strongly to each other but not to the substrate. This also explains why the film flakes off under mechanical impetus, such as gentle blowing or rins- 
ing, but does not redissolve in the PPA/EtOH solution. Literature research led to the identification of polyethylene terephthalate (PET) as a possible replacement. To confirm suitability a simple wetting test was conducted with DI water, the results of which are shown in Figure 3.17.

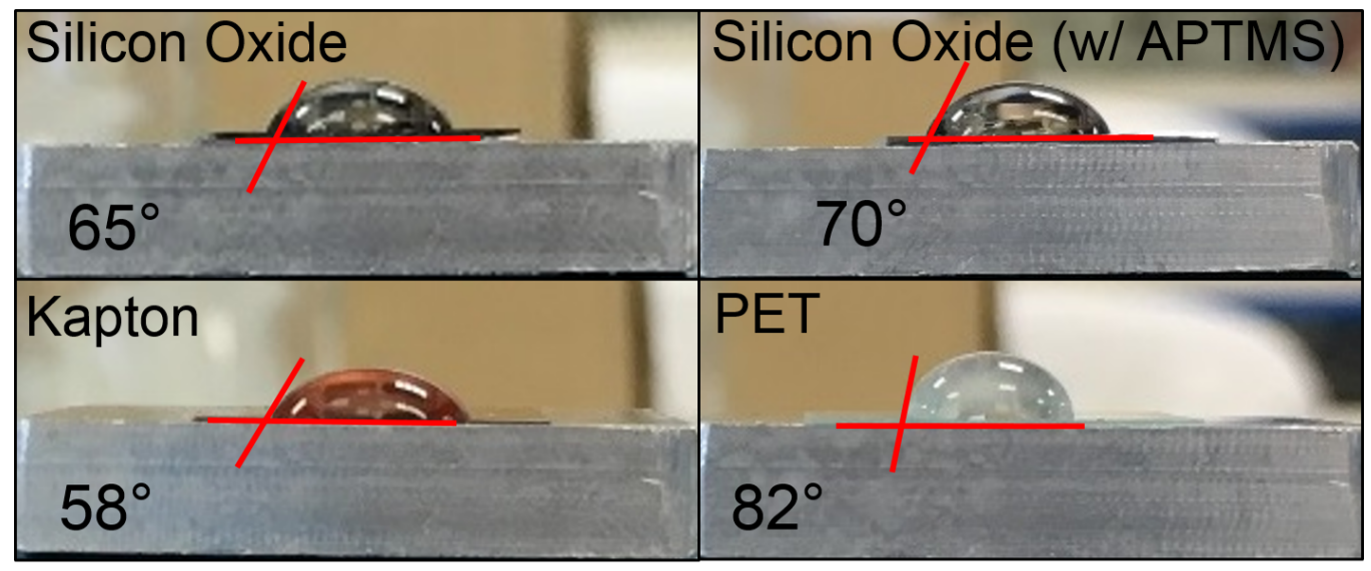

Figure 3.17: Wetting angles for DI water when $20 \mu$ 
(Figure 3.26) which limits the area over which you can accurately use a photolithographic technique. Using $10 \mu \mathrm{m}$ IDE spacing the success rate for device fabrication with all four pixels working was only about $50 \%$, and for any spacing smaller than this it was not possible to create a device with all 4 pixels not shorted.

\subsubsection{Room Temp Flexible Device}

Combining the PET substrates with an optimised inorganic ligand exchange led to the devices reported on in the rest of this Chapter. In order to show that bandgap tuning would be effective over the MIR a number of monodisperse samples were needed in order to demonstrate the differing detection of signals from neighbouring pixels with varying bandgaps. Figure 3.18 shows the FTIR spectra of the four samples used in the final devices reported here, as dried on the ATR crystal. The $\mathrm{C}-\mathrm{H}$ stretch of the organic ligand (near $0.36 \mathrm{eV}$ ) has been removed for clarity and each spectrum shows a clear excitonic peak, including the smallest bandgap sample at $\mathrm{Eg}=4.3 \mu \mathrm{m}$. 


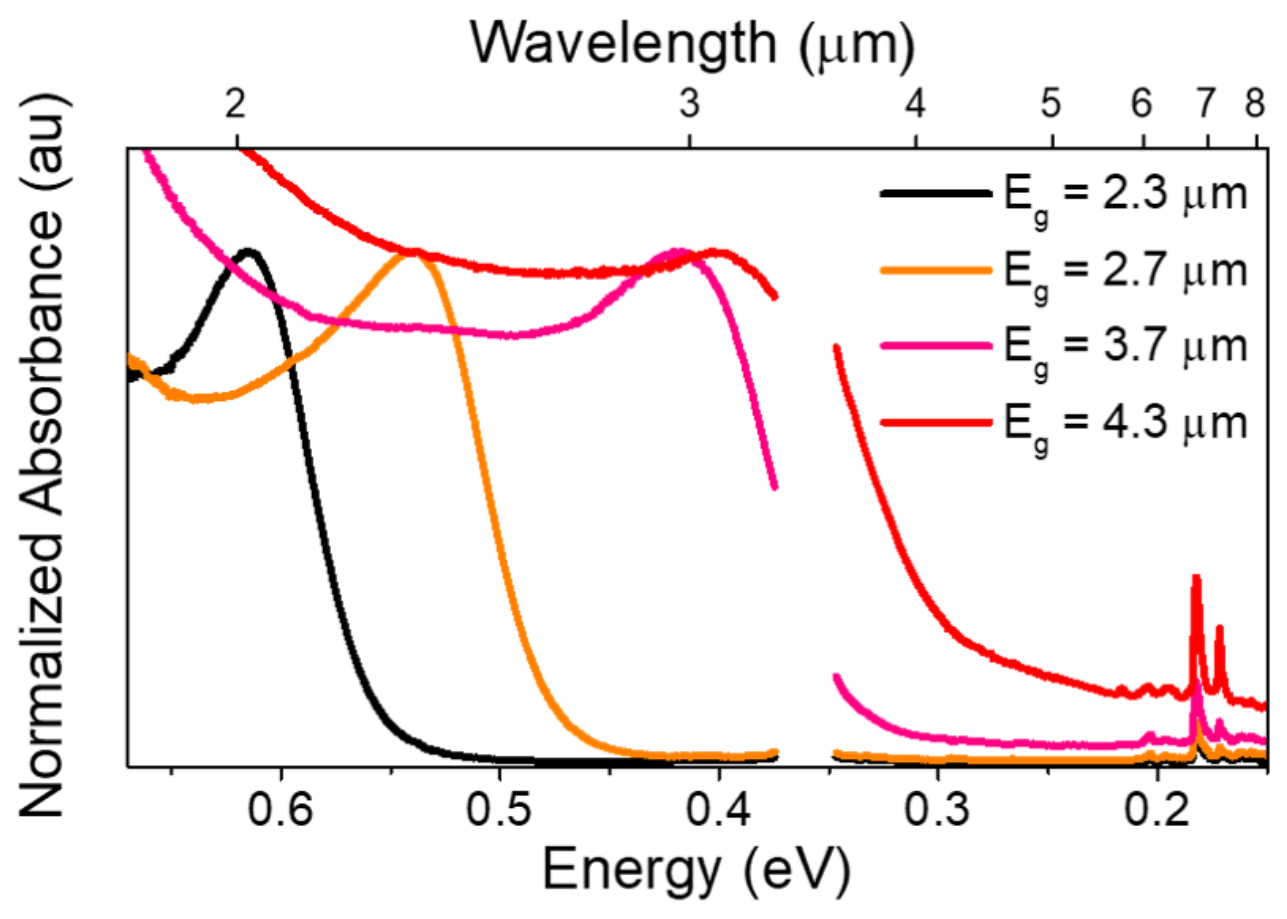

Figure 3.18: IR absorbance spectra of the NCs used in flexible devices reported on in the remainder of this Chapter. The spectra shows the colloid film dried on the ATR crystal, without ligand exchange. The stretch of the $\mathrm{C}-\mathrm{H}$ molecular vibration at $\approx 0.36 \mathrm{eV}$ has been removed.

Figure 3.19 shows the absorption spectrum of the same NC sample on an IR transparent glass with and without ligand exchange. The spray deposition method retains the characteristic 'excitonic peak', which in direct bandgap semiconductors indicates that the NCs have retained their nanoscale dimensions [137]. The term exciton used in the literature is somewhat misleading, as in strongly confined systems like that seen here the peak represents the transition from the valence band to the lowest excited state, as described by Bawendi et al. [138]. This definition calls into question the validity of using a Tauc approximation for estimating the bandgap energy, published work specifically in this area shows that using the bulk direct factor in the Tauc approximation without a size depen- 


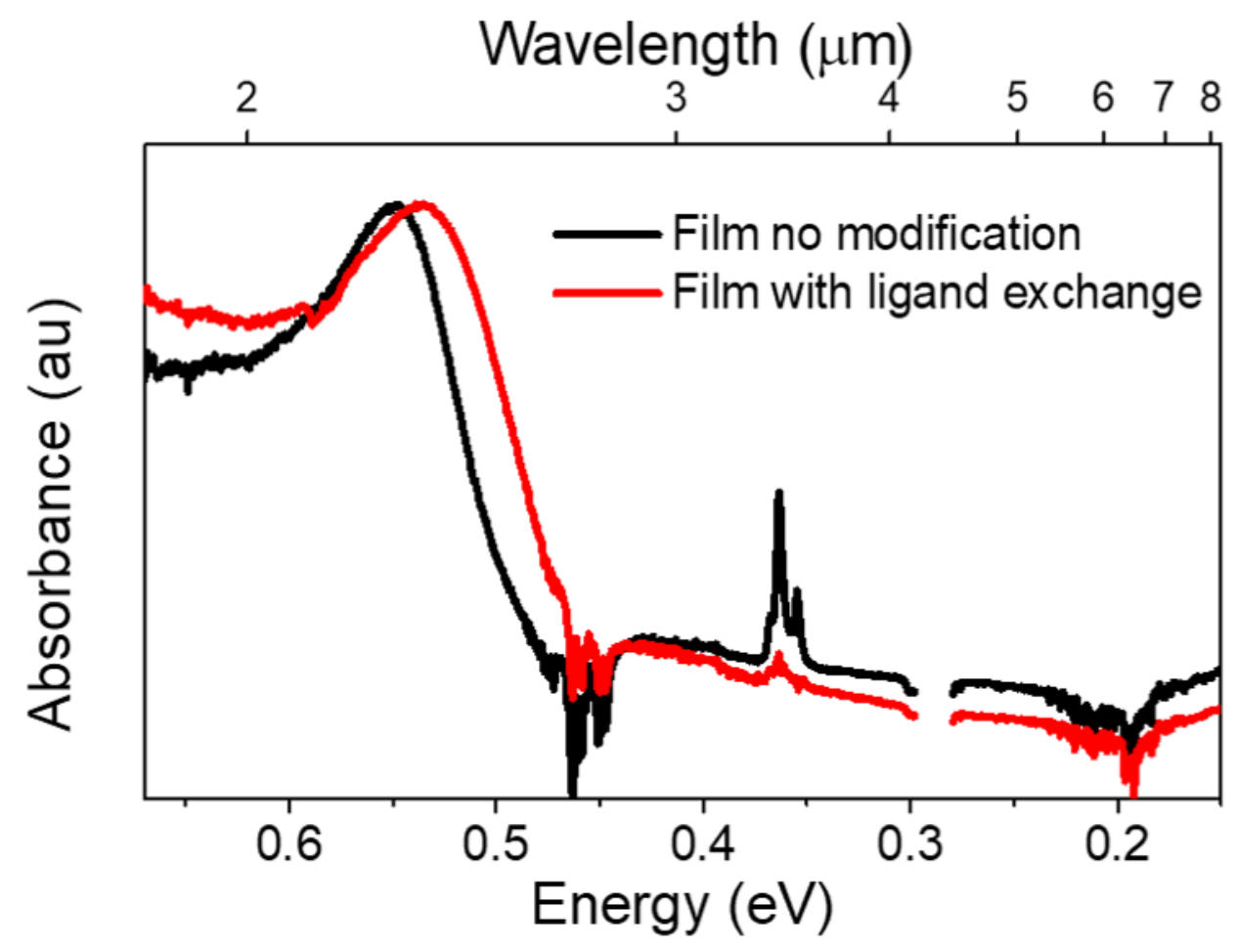

Figure 3.19: The absorbance change (redshift) of a film after the inorganic ligand exchange. The characteristic peak has been preserved(discussed in text) and there is less observed C-H stretching (peak at $0.36 \mathrm{eV}$.

dent power factor can lead to errors of up to $15 \%$ for NCs with a radius less than $5 \mathrm{~nm}$ [139]. Further work in the HgTe field is required to give the correct Tauc power approximation. The ligand exchange with $\mathrm{As}_{2} \mathrm{~S}_{3}$ after deposition was adapted from Tang et al. [111]. Following the ligand exchange there is a slight redshift of the peak. This is either caused by a relaxation of the quantum confinement due to wavefunction overlap between neighbouring NCs [92,140], or as a result of increased aggregation and NC sintering within the film [36]. In this case the excitonic peak indicating the lowest energy transition in the NC, has been preserved and is moved to slightly lower energy. This is best visualised as each NC getting slightly larger, which is the reason that the relaxation of confinement argu- 
ment is found most frequently in literature. However, of greatest importance from a device perspective is that there is only minimal degradation in the sharpness of the peak.

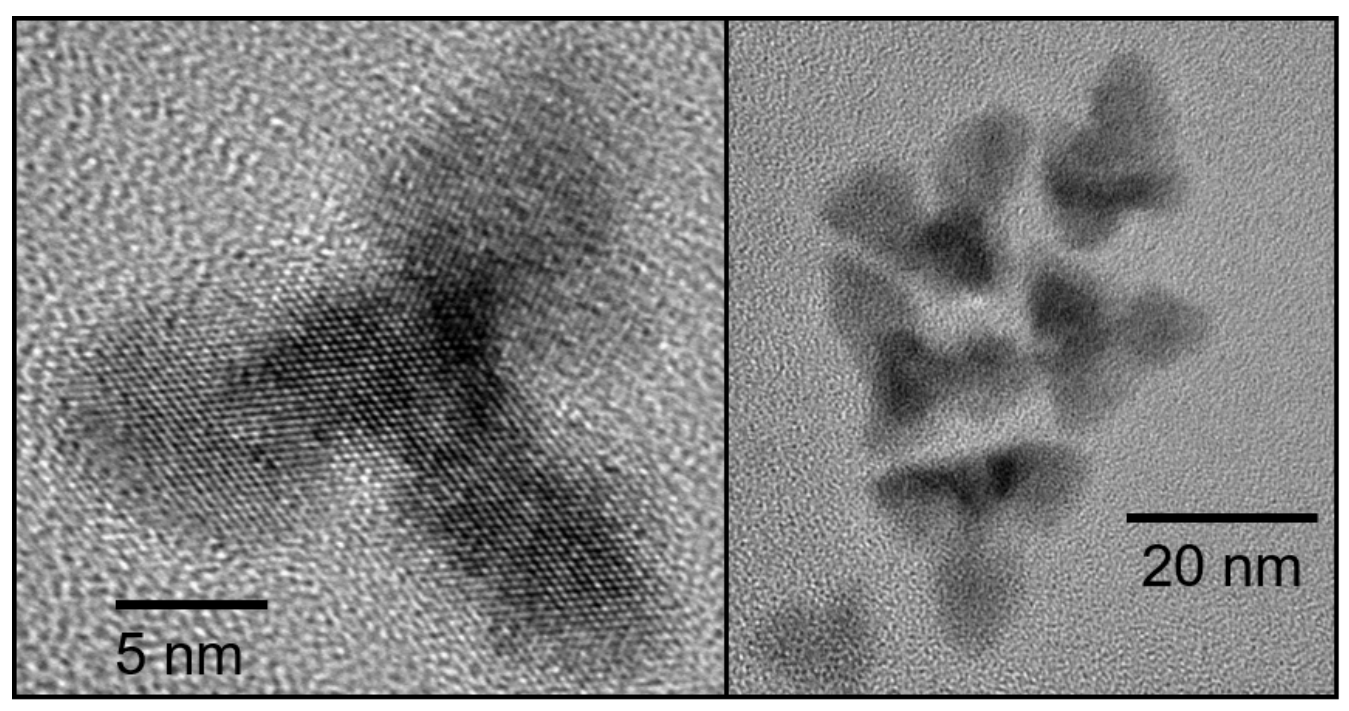

Figure 3.20: HRTEM (left) and TEM (right) images of the $4.3 \mu \mathrm{m}$ bandgap NC sample, showing clear lattice fringes.

The NCs are tetrapods, as shown in Figure 3.20, and the size was measured along the longest axis of each tetrapod. TEM images for each sample are shown in Figure 3.21 and the size distribution of each sample is shown in Figure 3.22. The size to band edge relationship differs slightly from previous reports of tetrapodal HgTe QDs [100], lying closer to that calculated for spherical HgTe QDs [141]. 


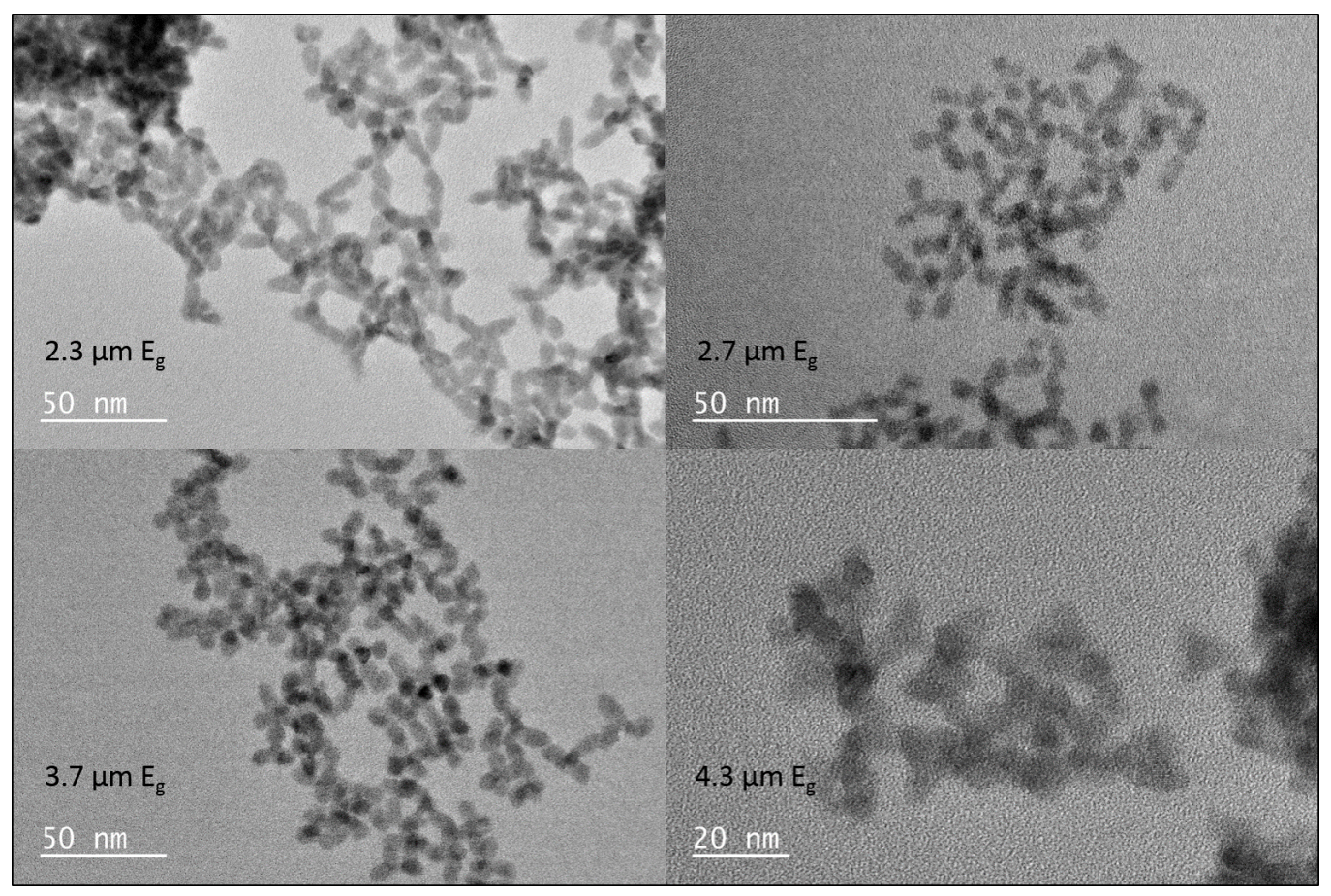

Figure 3.21: TEM images of all 4 of the samples used in this device, the bandgap of the sample is annotated in the bottom left. 

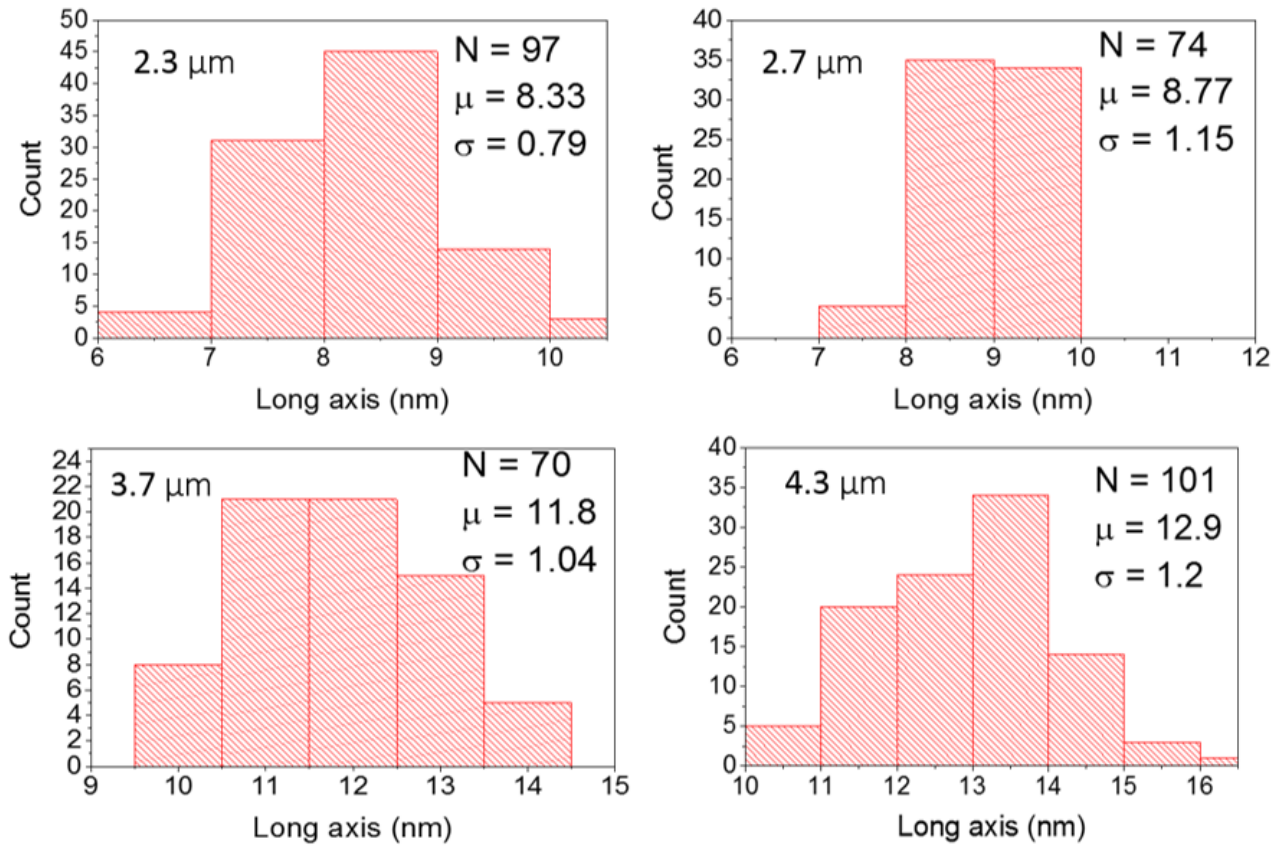

Figure 3.22: Histograms showing the size distribution for each sample, measured along the long axis. Where the measured bandgap of the sample is shown for each sample in the top left. 
Spray coating the DDT capped NCs was facile. To fabricate these devices, a stencil was made from $1 \mathrm{~mm}$ aluminium sheeting and this was clamped down onto the device. A key advantage of the PET substrates is that they are highly robust and are not affected by the pressure exerted by the stencil mount. The colloidal NCs in chloroform are then sprayed onto the device with an air brush on a nitrogen line. It was found that the ideal distance is approximately $10 \mathrm{~cm}$ as this allows the chloroform to evaporate before hitting the substrate. The entire mount assembly is then placed into the ligand exchange solution, rinsed and then dried with nitrogen. The fabrication method is summarised in Figure 3.23. The performance metrics below are all quoted for IDE structures with a $10 \mu \mathrm{m}$ trench length. 

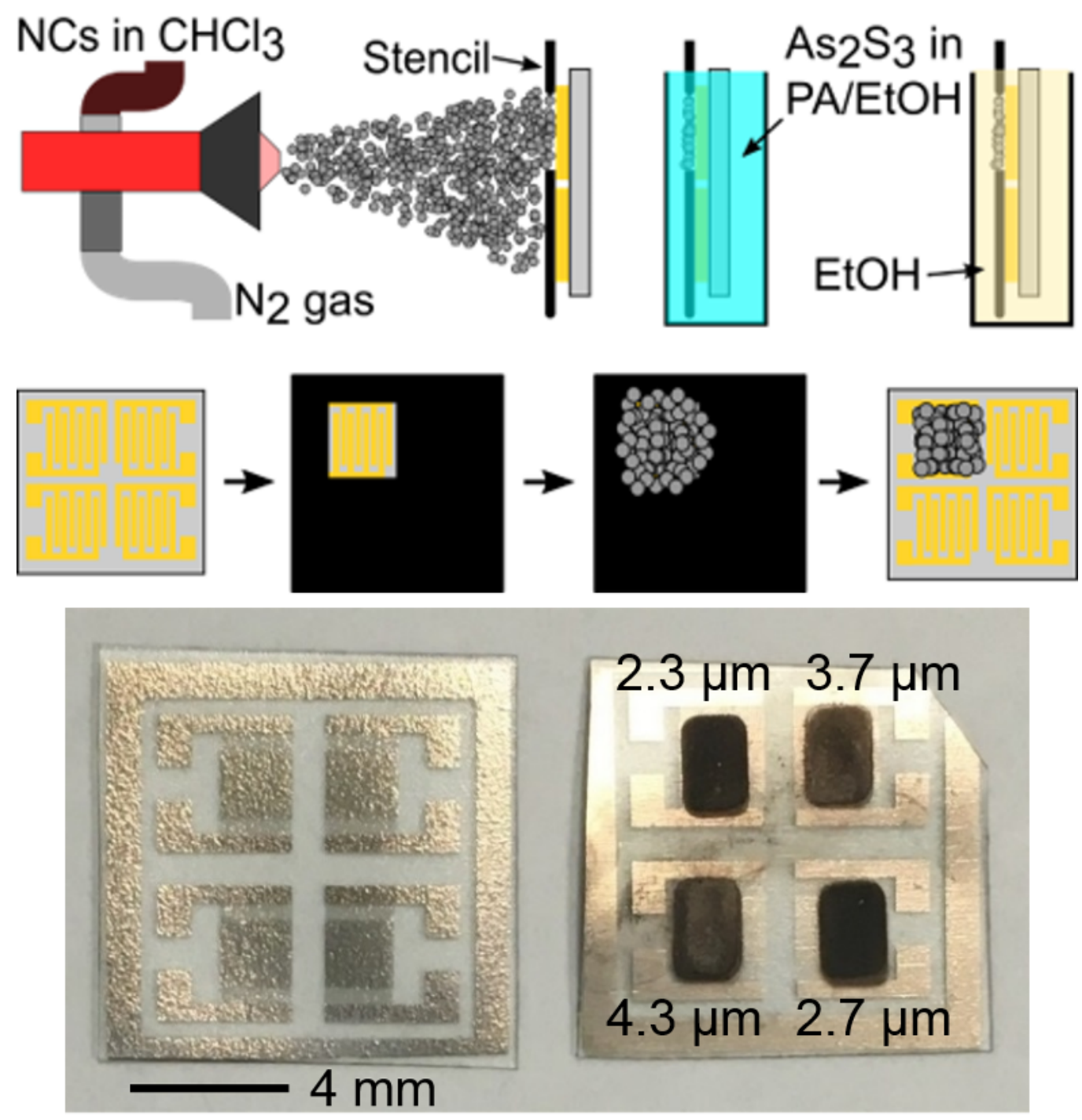

Figure 3.23: Cartoon of device fabrication process, three simple steps all conducted in air. Includes face on cartoon of the device at every step. The lower half of the figure shows an uncoated PET substrate on the left and a finished device on the right with the bandgaps of each pixel indicated.

The SEM image in Figure 3.24 shows the macro surface roughness of the PET to be $\approx 1 \mu \mathrm{m}$ [136]. Importantly EDS mapping of this area, shown in Figure 3.25 demonstrates that the QDs have evenly covered the electrodes. In terms of the film itself the AFM scan in Figure 3.26 shows 


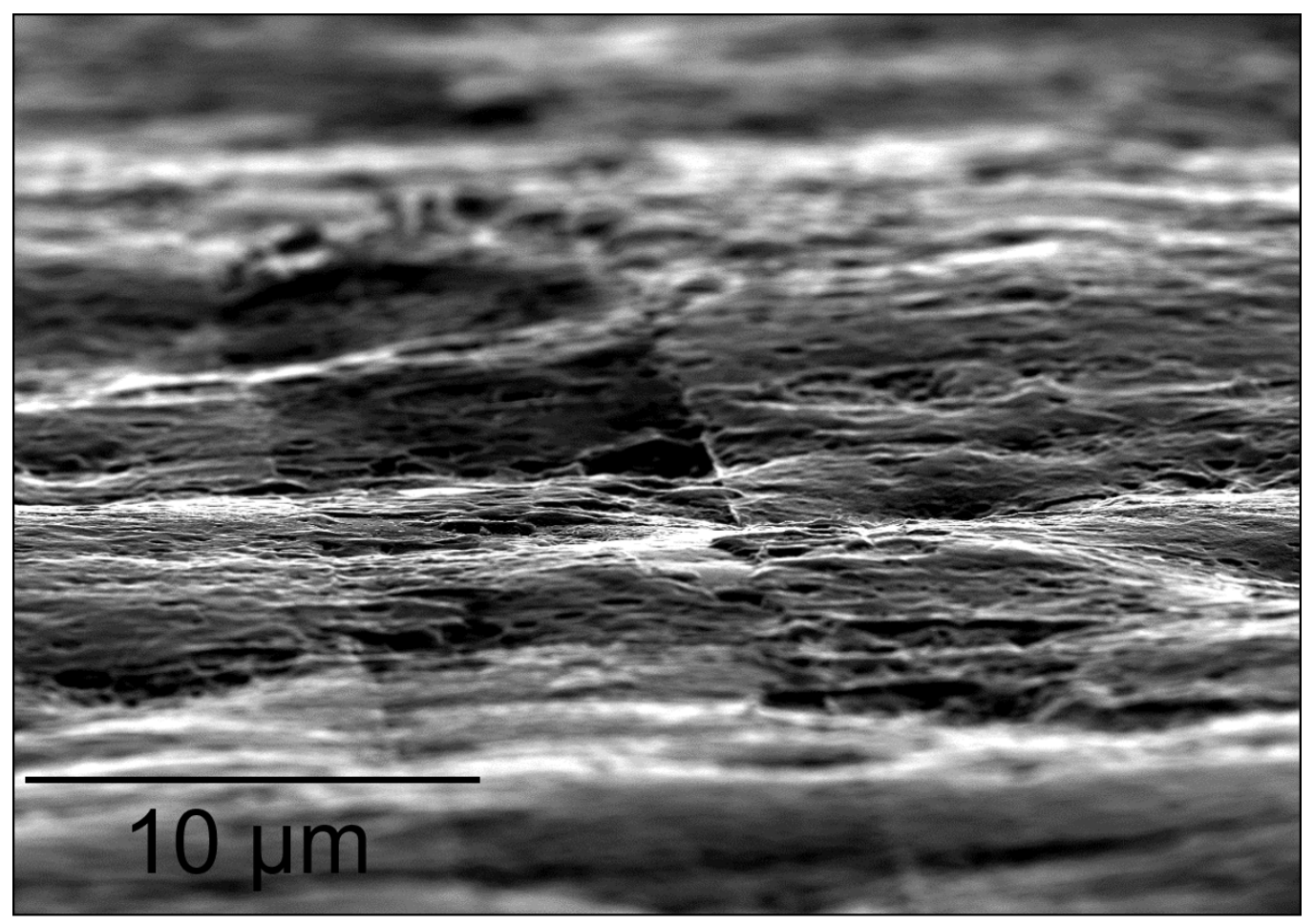

Figure 3.24: SEM image of inorganically treated HgTe QD film covering a trench between two IDEs, notice the large scale surface roughness of the PET. The smaller scale surface roughness is the $Q D$ film. This is a close up of the finished device shown in Figure 3.23 .

that the local surface roughness over a $1 \mu \mathrm{m}$ square is on the order of $50-$ $100 \mathrm{~nm}$. 

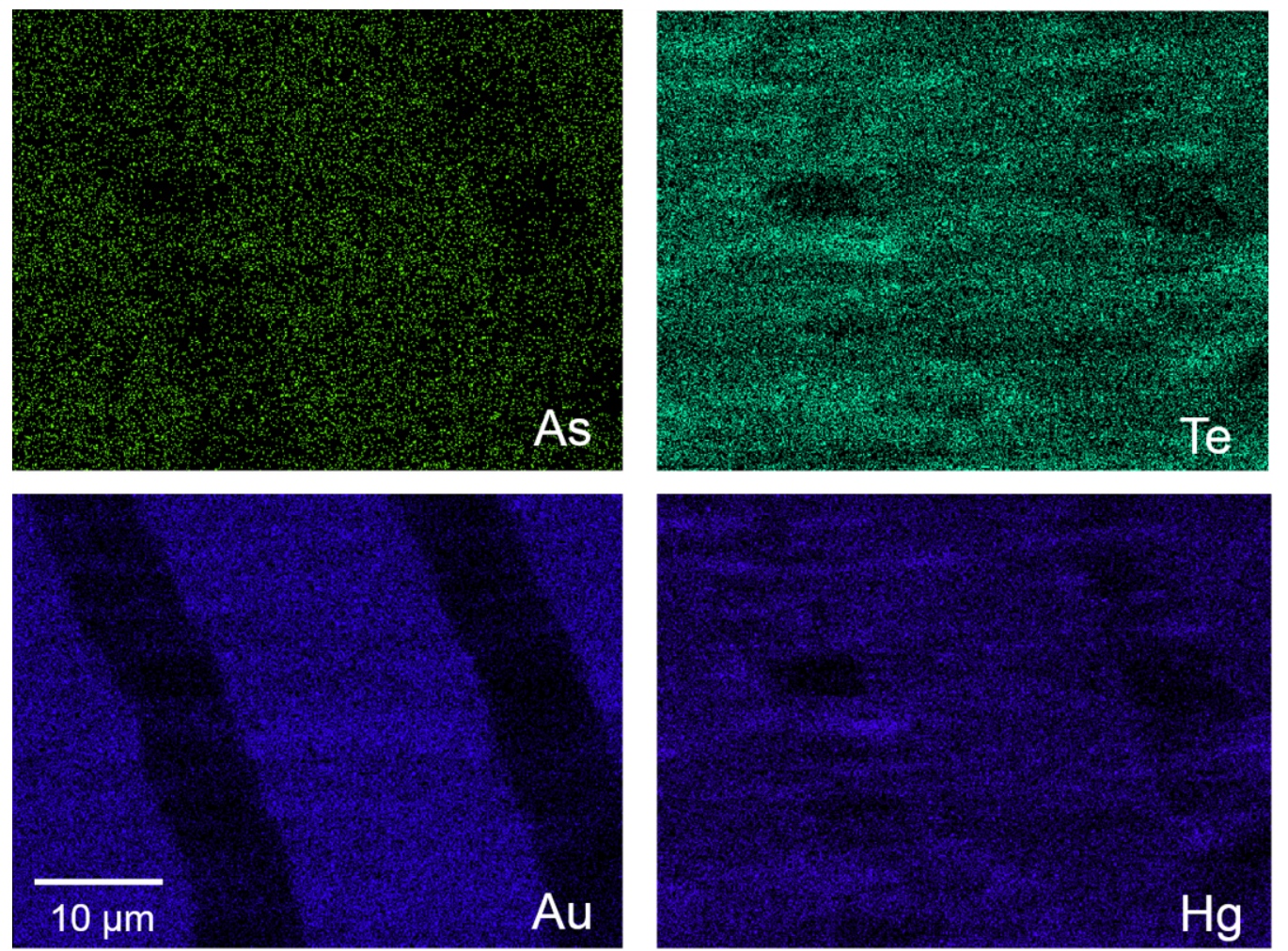

Figure 3.25: SEM EDS map of a film covering two trenches. These are highlighted with the Au map. It can be seen from the Te plot that the film has evenly covered the surface. It is important to note that there is some line overlap between $\mathrm{Hg}$ and Au, therefore the Te coverage offers the greatest insight into the film coverage.

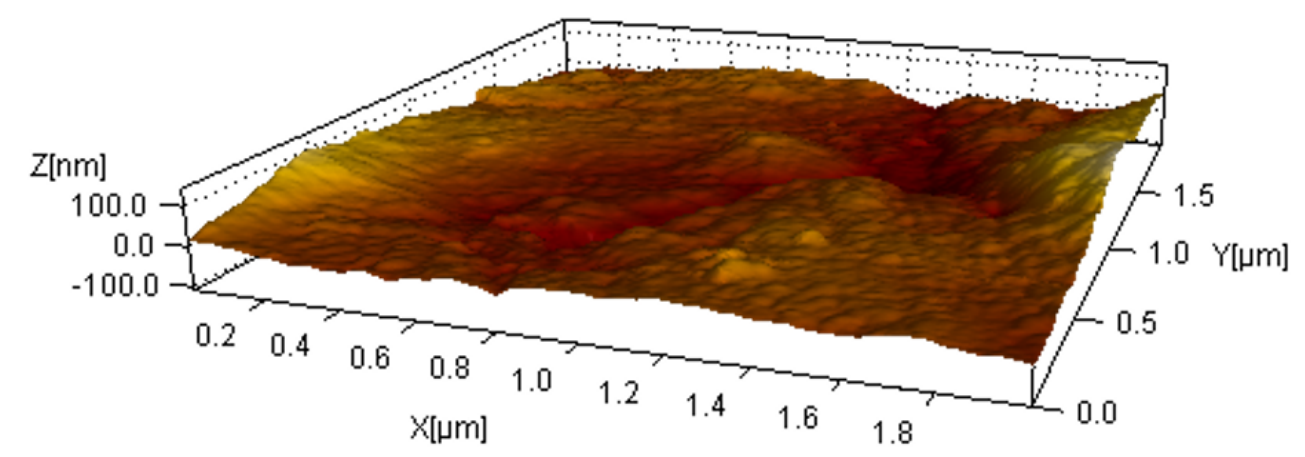

Figure 3.26: AFM image of the film on top of the PET substrate. As can be seen from the $z$-axis the local surface roughness is on the order of 50-100 $\mathrm{nm}$. 


\subsection{Discussion}

For the final flexible, room temperature device, samples were mounted in an uncooled cryostat with sapphire windows, which act as a LWIR filter. They were then excited via blackbody (BB) emission passed through varying longpass filters, as indicated in the figure, with an optical chopper used for time resolved measurements. The details of this methodology are covered in Chapter 2. Figure 3.27 shows the responsivity values for each pixel with each filter that permitted above-bandgap excitation. All reported values are for films spray coated onto PET substrates. The values

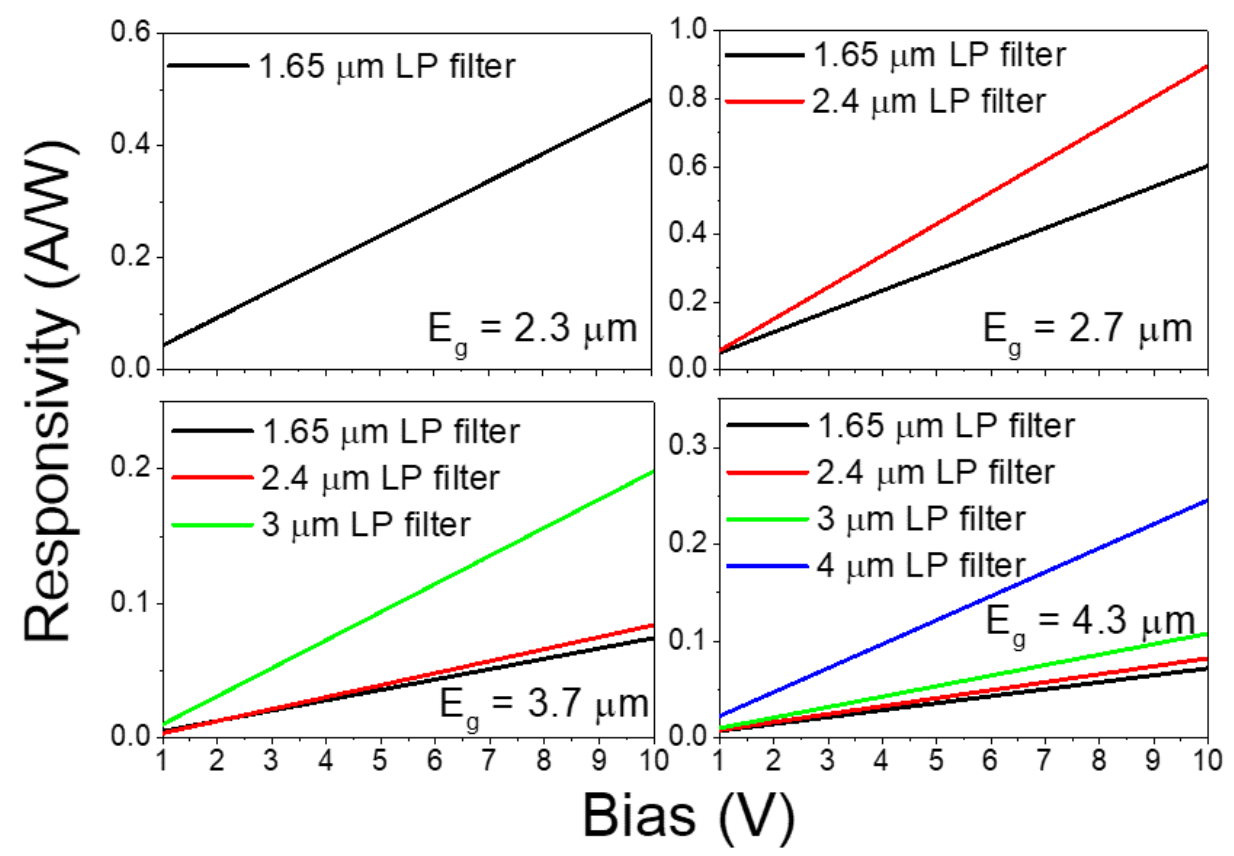

Figure 3.27: The responsivity of each pixel to various spectral selections of incident radiation at $5 \mathrm{~Hz}$. The BB target is at $1073 \mathrm{~K}$.

obtained for the $2.7 \mu \mathrm{m}$ pixel with a $2.4 \mu \mathrm{m}$ longpass filter in place reach $0.9 \mathrm{~A} \mathrm{~W}^{-1}$ at $10 \mathrm{~V}$ bias. This compares favourably to other devices using the same planar architecture. For example other groups have reported 
a phototransistor with a responsivity of $0.5 \mathrm{~A} \mathrm{~W}^{-1}$ at $12 \mathrm{~V}$ under similar conditions, although it should be noted the superior time response of this device gave it a higher detectivity [110]. For planar devices, $0.1 \mathrm{~A} \mathrm{~W}^{-1}$ at $2 \mu \mathrm{m}$ [111], and $0.22 \mathrm{~A} \mathrm{~W}^{-1}$ at $7 \mathrm{~V}$ with a $3 \mu \mathrm{m}$ cut off have also been reported [99]. The other pixels in our devices achieved 0.5, 0.2 and 0.4 $\mathrm{A} \mathrm{W}^{-1}$ for the $2.3 \mu \mathrm{m}, 3.7 \mu \mathrm{m}$, and $4.3 \mu \mathrm{m}$ pixels, respectively. Figure 3.27 shows that the responsivity increases as the spectral filter approaches the bandgap of the material. This demonstrates that multiple pixels of varying band-gaps can provide more responsive coverage of the MIR spectral region. The reason for this behaviour is not explicitly known, however if the incident radiation is a close match to the excitonic peak it would be expected that efficiency would be increased due to a reduction of hot carriers in states above the conduction band ground state [142].

Using the photocurrent generated by each pixel it should be possible to reconstruct the incident spectra. In essence, the photocurrent should drop to zero once there are no above bandgap photons hitting that pixel. This does occur and is shown in Figure 3.28. Even in the cases where the bandgap is only $300 \mathrm{~nm}$ less than the shortest wavelength of the incident (longpass filtered) radiation, the photocurrent drops to zero. The device can then be described as having a spectral sensitivity in the MIR of $300 \mathrm{~nm}$, or approximately in the range of 60 to $30 \mathrm{meV}$, at 2.3 to $4.3 \mu \mathrm{m}$ respectively. The noise spectral density (NSD) of the $2.3 \mu \mathrm{m}$ pixel is shown in Figure 3.29. The values are similar but slightly higher than other reported devices [110], ostensibly from processing in air. At no point did the measured noise drop below the calculated thermal noise floor or shot noise floor [143]. The noise trends inversely with frequency as has been shown before for these films $[43,66,110]$. Following from this the specific detectivity of the pixels can be calculated and this is shown in Figure 3.30. This was done for each pixel by finding the NSD at $5 \mathrm{~Hz}$ and at $10 \mathrm{~V}$ and using this as the noise current for each pixel. 


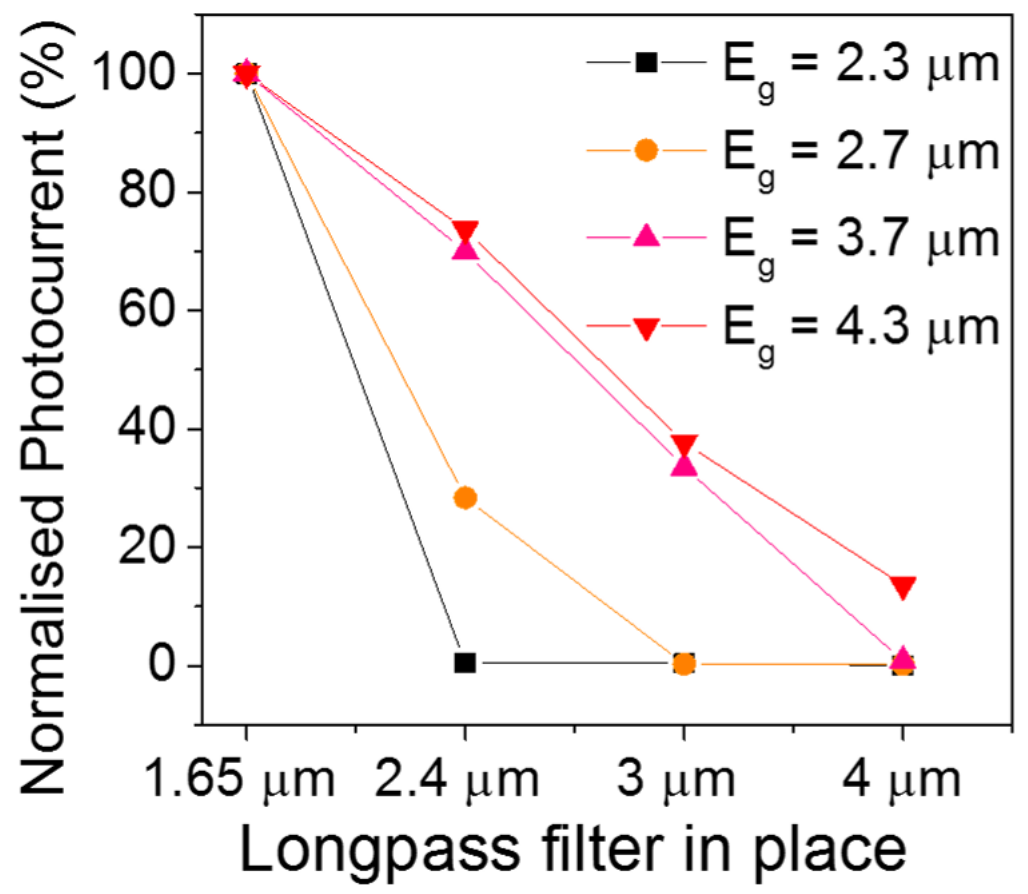

Figure 3.28: Normalised photocurrent of each pixel plotted against the filter in use. The photocurrent is able to detect the "colour" of the incident radiation with at least $300 \mathrm{~nm}$ sensitivity. 


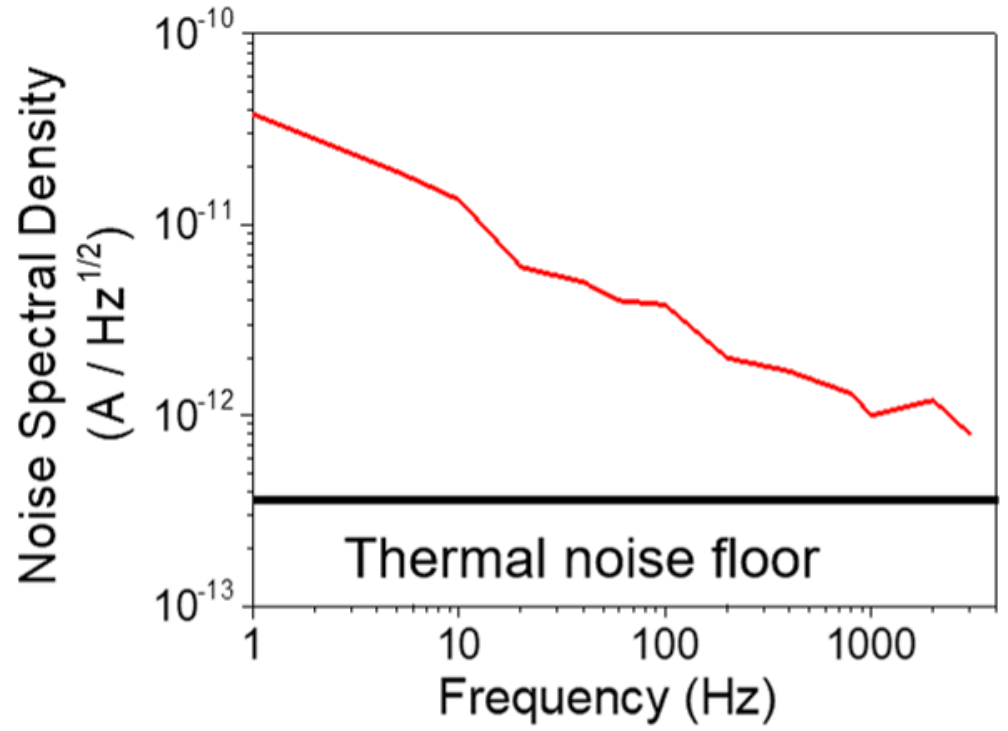

Figure 3.29: Noise spectral density of the $2.3 \mu \mathrm{m}$ bandgap pixel, with the thermal noise level indicated. This data was collected at $10 \mathrm{~V}$ driving voltage across the frequency range shown.

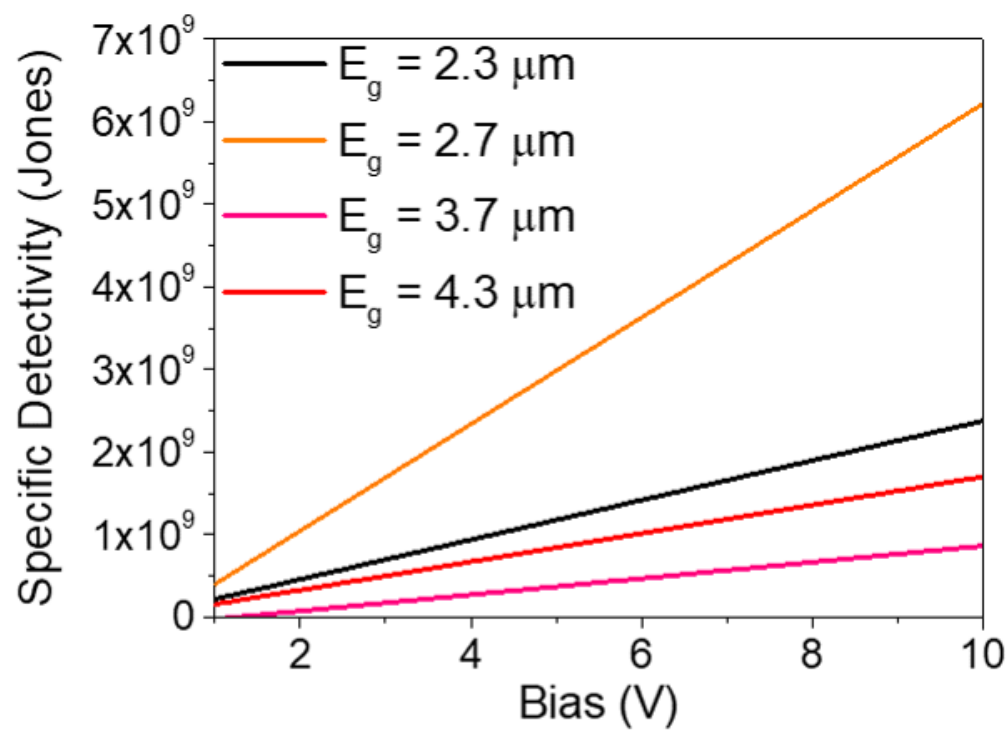

Figure 3.30: Specific detectivity of each pixel, calculated from the responsivity and the noise at $5 \mathrm{~Hz}$ with the relationship $D^{*}=\frac{R \sqrt{A}}{I_{n}}$. 
The time response of the device is vitally important if it is to be useful for real world imaging applications and air-processed films normally do not perform well on these metrics. Lhuillier et al. [99] demonstrated the need to conduct inorganic ligand exchanges in air-free conditions to prevent the creation of surface traps. These traps manifest themselves as either a slower time response in the best case or, as an unresponsive highly conductive film. In Chapter 4 we show new research that oxygen derived trap states also cause hysteresis and memristive behaviour in organically capped films. However here the ligand exchange process could be optimised, in air, to create devices that functioned well out to $200 \mathrm{~Hz}$. Since the frame rate of micro bolometers is 60 frames per second (FPS) for the best devices [144], and 30 FPS in cheaper uncooled devices [133], operation above $120 \mathrm{~Hz}$ therefore represents a significant milestone. The inset to Figure 3.31 shows the frequency response for the $2.3 \mu \mathrm{m}$ pixel with a chopped BB source. Clearly this material can operate at speeds that are suitable for video capture, even if the time response is below that of air-free devices. The time response was characterised using a laser diode, and shows two clear decay pathways. The faster decay with a time constant of $264 \mu \mathrm{s}$ is the bi-molecular recombination, which predicts that, with reduced surface trapping, the air-processed films could be capable of imaging at over 600 Hz. [110] The slow decay curve represents the trap-assisted recombination pathways and has a time constant of $20 \mathrm{~ms}$. Film processing in air would be expected to cause a large number of oxygen derived trap states and as such this very slow trap mediated pathway represents the difference between the use of air-free and air-processed HgTe NCs. Since the noise displays a $1 / \mathrm{f}$ relationship, the specific detectivity changes with the operating frequency, reaching a value of $8 \times 10^{9}$ Jones at $200 \mathrm{~Hz}$ and $293 \mathrm{~K}$. This is shown in Figure 3.32. This value is lower than photo-transistors operating at $2 \mathrm{KHz}$ [110] and sensitised 2-D transistors [109] but is equivalent to previously reported planar CQD devices. Our fabrication method was also tested with various feature sizes using stencil features as small 


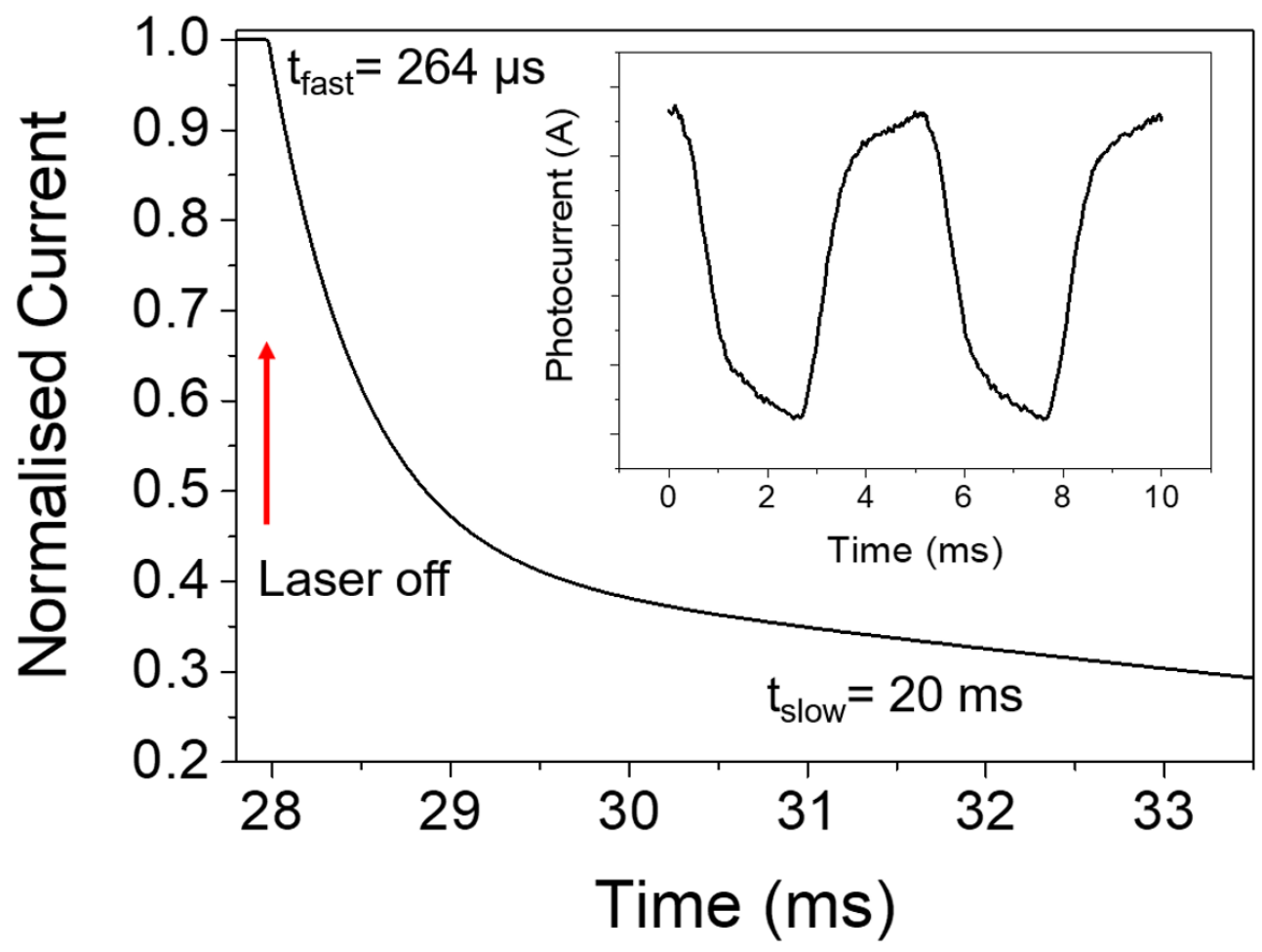

Figure 3.31: Transient photoresponse for a laser diode source, showing a fast and a slow decay. The fast decay is bi-molecular recombination with the slow decay due to trapassisted recombination. Inset shows the measured time response to a $200 \mathrm{~Hz}$ chopped BB source.

as $0.4 \mathrm{~mm}^{2}$. The responsivity for this small feature size fabricated with the $3.7 \mu \mathrm{m}$ bandgap NCs is shown in Figure 3.33 along with the NSD (inset). The responsivity is very similar to that shown in Figure 3.27 proving that the performance of the films is unchanged with feature size. Additionally, the NSD is lower, as would be expected for a smaller surface area device, noting that the thermal noise floor is again, not breached. 


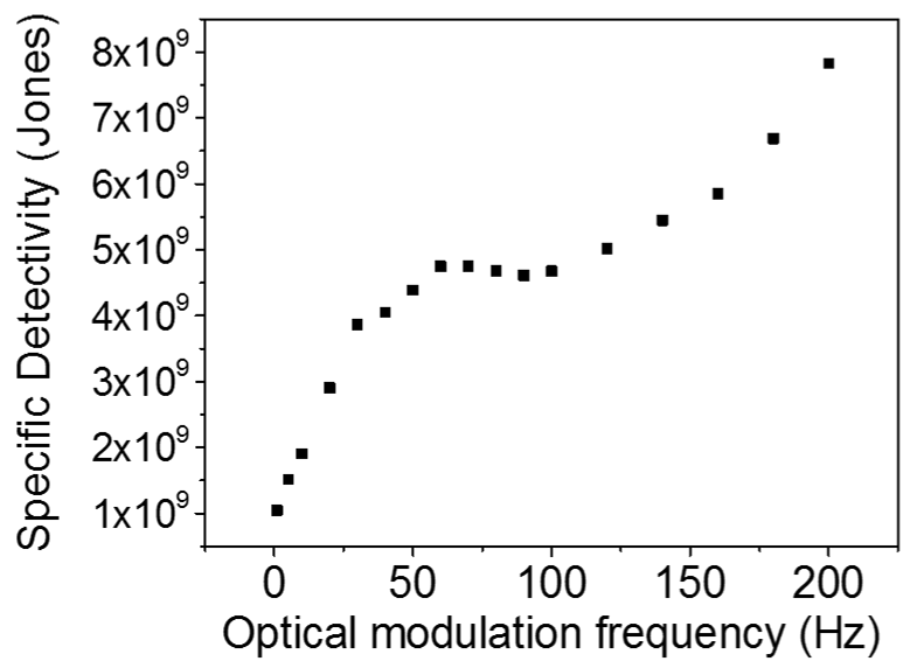

Figure 3.32: Specific detectivity with increasing incident frequency for the $2.3 \mu \mathrm{m}$ bandgap pixel, at $10 \mathrm{~V}$ bias. The dip at $75 \mathrm{~Hz}$ is caused by the responsivity decreasing relatively faster than the noise between 50 and $100 \mathrm{~Hz}$. 


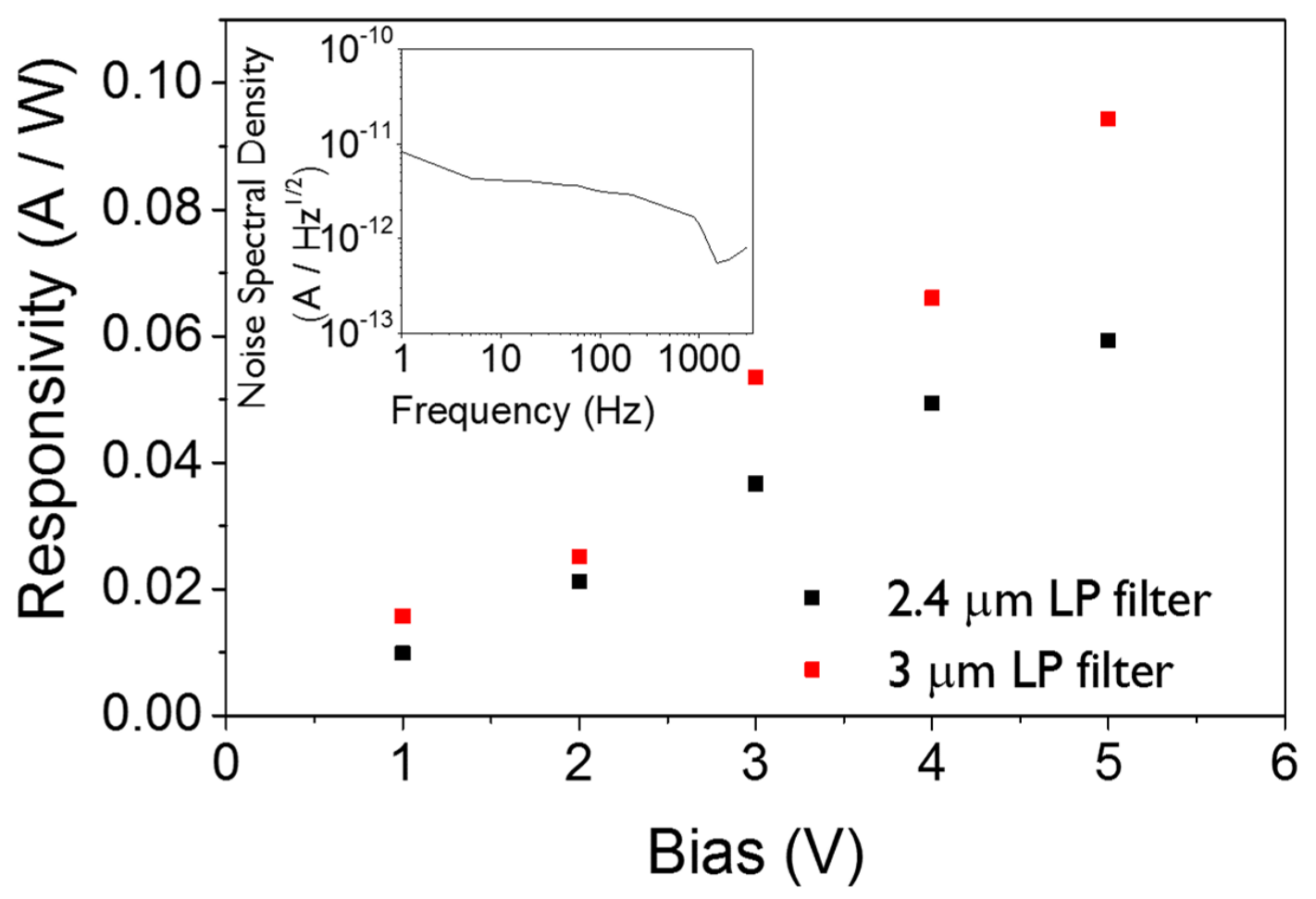

Figure 3.33: Performance of a smaller pixel with an area of $0.4 \mathrm{~mm}^{2}$ made with the 3.7 $\mu \mathrm{m}$ bandgap colloid. Responsivity is very similar to Figure 3.27. The inset shows the NSD for this device which is lower due to the scaling effect of area on device noise. 
The PET substrates were initially chosen for their robustness and low cost, however as they are also flexible the device behaviour after deformation was tested. This was measured by bending the substrate to the indicated angle then measuring the conductivity when it returned to normal. Devices display considerable resistance to structural deformation, with conductance remaining nearly unchanged $(<5 \%)$ after bending to angles of up to $45^{\circ}$, as shown in Figure 3.34. As well as the dark conduc-

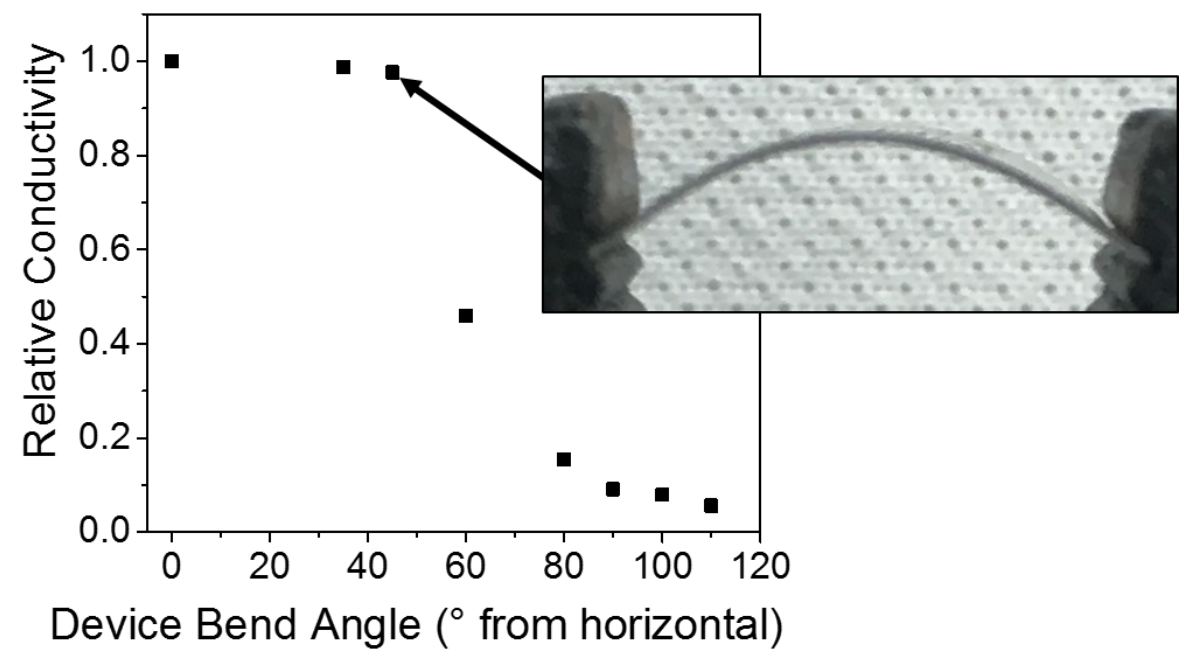

Figure 3.34: Plot showing the relative (dark) conductivity of the device after it has been flexed to the angle indicated. Measurement is on the device placed back flat on the sample mount, therefore the cracks in the film caused by the flexing are permanent.

tivity the photocurrent was also measured after each deformation and this showed that relative photocurrent percentage was unchanging with deformation. This suggests that large scale defects are being introduced into increasing percentages of the film with increasing angle, leaving an ever smaller portion of the film that can conduct between electrodes, exactly what is expected to occur with a brittle film. 


\subsection{Conclusion}

In conclusion we have demonstrated a colloidal NC device that highlights the strengths of colloidal NCs for photodetection technologies. High responsivities up to $0.9 \mathrm{~A} \mathrm{~W}^{-1}$ were shown beyond $2.5 \mu \mathrm{m}$, with a significant time response at $200 \mathrm{~Hz}$ achieved using an MIR (instead of visible) source and displaying a competitive specific detectivity of $8 \times 10^{9}$ Jones at $200 \mathrm{~Hz}$ and $293 \mathrm{~K}$. Bandgap tuning by changing the NC size is easily transferable to high performing devices that can be fabricated quickly, easily and very cheaply. The performance of these devices compares favourably to other published devices $[43,54,94,99,110,111]$ and known fundamental issues with air-processed materials have been mitigated. As such, similar devices could feasibly be used for multicolour MIR video capture, without the need for any other filter layers, representing a significant step toward cheap, robust MIR imaging using spray coated colloidal NCs. Importantly we showed imaging potential at frequencies up to $200 \mathrm{~Hz}$ which is far in excess of that displayed by even the best uncooled microbolometers. These devices functioned very favourably when compared to literature values. In the author's opinion this is for three main reasons:

1. The intentional use of more $\mathrm{Hg}$ than $\mathrm{Te}$ in the synthesis increases the likelihood of a $\mathrm{Hg}$ rich surface. As the NCs are soluble in nonpolar solvents and are passivated by a de-protonated thiol the ligand bonding must be primarily at dangling cation sites $\left(\mathrm{eg} \mathrm{Hg}^{2+}\right.$ or $\left.\mathrm{Hg}^{+}\right)$. Therefore an $\mathrm{Hg}$ rich surface would increase the ligand density, leading to an increase of colloidal stability and a increased likelihood that the QDs that reach the substrate are still relatively well passivated. Higher levels of surface passivation have a twofold effect, leading to fewer surface trap sites and less aggregation. The former increases the fast component of transient optical measurements and the latter ensures that the bandgap remains distinct, leading to high spectral sensitivity. 
2. Spray coating onto PET seems to be an excellent method for depositing hydrophobic QDs. Once deposited the QDs are well adhered to the surface, and the trenches are well filled, leading to a large number of conductive pathways; it is likely that this effect could be further enhanced by a surface treatment of the gold. The spray coating removes the solvent from the film during deposition as it has evaporated before the film is formed, therefore once each QD is deposited it will not be redissolved in solvent at any point. This is not the case when fabricating, for example, with a three layer spin coat, as in that situation the solvent from the second layer can interfere with the first layer. This could increase inhomogeneity in the films, above that which has already occurred during the ligand exchange.

3. The samples are relatively monodisperse, which ensures that the range of bandgap energies within the final ensemble is small and the trapping within the film is limited to a specific oxygen trap rather than a large array in energy of effective trap states caused by size dispersity. This is also the reason for what is inarguably high speed performance for oxygen polluted QDs. Reducing the trap population forces bi-molecular recombination and leads to an improved time response. This monodispersity in the film is likely due somewhat to the two items above, but also to a heat up synthesis that was properly optimised.

There are a number of obvious next steps that emerge from this work. From a device engineering side it is clear that the pixel size must be reduced so as to go from a device that shows material proof of concept to a device that shows imaging proof of concept. If the intent is to show a number of pixels working simultaneously then the issues with photolithography on PET will also have to be addressed, either by finding a way of smoothing the PET surface or by using a different material for the substrate. The large amount of work that went into optimising the film to 
get the results shown here also suggests that delivering a material that is electrically uniform across multiple pixels will be a challenge. Solving the uniformity issue can be addressed either as a fabrication issue or a surface passivation issue. The former would aim to create multiple areas of uniform film thickness and QD density, the latter would aim to reduce film to film electrical variation by having the most uniform passivation possible, either in the colloid or in the solid. From a materials research perspective there are still a huge number of avenues for further work. The most insightful would be a study based solely around the electrical behaviour of films, particularly with regard to the interplay of ligand type, concentration and film parameters on basic electrical behaviour. Developing a thorough understanding of the films may seem like work that should have been a building block, but in this relatively new and fast moving field an exhaustive understanding of the interplay between film deposition parameters and treatments and the correspomnding electrical response does not yet exist, even though it would be invaluable in creating high performing devices. In the next Chapter we do our part in addressing this by developing an understanding of how the devices behave electrically as they spend time in air. 


\section{Chapter 4}

\section{Memristance in Oxidised HgTe Devices}

This work is published in:

Photo-Electrosensitive Memristor Using Oxygen Doping in HgTe Nanocrystal Films, Cryer, M. E.; Fiedler, H.; Halpert, J. E. ACS Appl Mater E Interfaces, 2018.

\subsection{Motivation}

The work in this Chapter was motivated by a desire to more fully understand the electrical response seen in films of the organically treated QDs reported in Chapter 3. What began as a study into the behaviour of the hysteresis observed while sweeping the voltage, led to the characterisation of the transient current response to applied voltage in the films. An increased understanding of the behaviour of mobile charges within these films is necessary to design better devices. However, the time dependent response to injected charge, or output dependence on charge history, observed in these films, meant that the films had the potential to behave as photosensitive memory devices. Our work builds on the only other 
similar published work in the literature which was the work on charge history in CdSe reported by Fischbein et al. in 2005 [145]. The history of memristive behaviour with respect to reports of behaviour of this type in novel material systems is given briefly below. The study of memristive behaviour in NC films is worthwhile due to the possibility of producing devices that can function as photodetectors, transistors and memory circuits in one device and can then be further integrated with silicon photonics [30]. This approach could solve the silicon size limit problem, in some areas, not through further miniaturisation but by increased multifunctionality. Furthermore, the study of the time evolution of non-linear behaviour in the IV curves of NC films will inform understanding of both the behaviour of charges in the film and the chemical changes that affect long term stability of these devices in ambient atmospheres.

\subsection{Background}

As conventional circuit technologies reach their size limit, there has been increased interest in nanometer scale technologies that can either show comparable device metrics or deliver additional functionality at smaller scales [146]. Any use within this field of NC based devices is therefore of considerable interest. Recently there has been significant development in resistive switching and memristive behaviour in potential new materials for logic circuits, memory and neural networks [147-150]. While the existence of a true memristor has been fiercely debated, the general concept of memristance in passive devices is widely accepted [151-156]. A memristive device, often called a "memristor", describes any passive electrical component in which the output is dependent upon a system specific state variable [153]. In particular, many memristive devices use the recent history of charge flow, rather than the theoretically postulated magnetic flux [152], to modulate the current output of the device. In this arrangement, the memristive device shows memory of the previous mode of op- 
eration by operating in either a high (HRS) or low resistive state (LRS). Most reported memristive materials are based on high band gap metal oxide thin films [156]. However, colloidally synthesised semiconductor NCs can be expected to provide additional functional advantages due to their light sensitivity and the long history of sophisticated chemical control over their size, shape and surface functionalities [157-159]. Reports of electrical bi-stability in NC thin films started in the early 2000s but these displayed low on/off ratios and minimal overall change in resistance state [160-163]. These original bi-stable devices were observed using films that had been encapsulated in an organic matrix $[162,164]$, or had been sintered postdeposition $[165,166]$. Many thin film organic/NC systems did show effective memristive switching $[167,168]$, yet due to the fabrication methods employed it is difficult to ascribe the observed effects entirely to the NCs. In some cases organic molecules alone have been shown to make highly effective memory devices [169], and high temperature treatments can remove capping ligands, causing NC agglomeration or the formation of surface states. Since this time, there has been great progress in QD synthesis, device fabrication and measurement techniques [157-159]. Electrical switching and memory effects have been reported in some systems containing II-VI and IV-VI semiconductor NCs [145, 149, 170-172], and high on/off ratios have been observed for core-shell NCs [173]. Integrated carbon nanotube/NC devices have shown long term ambient environment stability [174], and low read/write voltage requirements have been demonstrated [167]. However no single device has met all of these criteria. Meanwhile, greater understanding of the behaviour of charges in NC films has not yet led to a consensus for a mechanism for memristive behaviour in colloidal NC devices [166,175-179]. To date, the lesser attention to colloidal NC memristance stands in stark contrast to the wellstudied mechanisms that underpin memristance in $\mathrm{TiO}_{2}, \mathrm{ZnO}$ and other oxide nanocrystalline and thin film systems [156, 180-183]. 


\subsection{Experimental}

$3.8 \mu \mathrm{m}$ bandgap HgTe NCs were synthesised via the method reported in Chapter 3, although these QDs were not synthesised with an excess of $\mathrm{HgCl}_{2}$ as the QDs used in the final devices reported in Chapters 3 and 5 were. All processing was done in air at ambient conditions of 290-295 K and $50-60 \%$ humidity.

\subsubsection{Devices}

The $300 \mathrm{~nm}$ oxide layer silicon IDE devices were lithographically prepared as reported in Chapter 3 . To briefly recap, the NCs were layer by layer spin coated onto the IDE (34 fingers per side, $2 \mathrm{~mm}$ long with $20 \mu \mathrm{m}$ spacing, top down device area is $5 \times 10^{-6} \mathrm{~m}^{2}$ ) devices at $4000 \mathrm{rpm}$ for $60 \mathrm{~s}$ with $3 \mathrm{x}$ $20 \mu \mathrm{l}$ drops applied once rotation had started. Default ramp settings were used. Post deposition, each layer was treated in an EDT/ $\mathrm{HCl}$ in $\mathrm{EtOH}$ (1:1(3.0M):20) solution to exchange the DDT capping ligands.

\subsubsection{Measurement}

Devices were characterised with an intensity controllable white LED lamp in air at room temperature or in a temperature controlled Janis VNF-100 optical cryostat, in either the dark or with illumination provided by a Thorlabs $670 \mathrm{~nm}$ laser diode, with $2.5 \mathrm{~mW}$ incident on the sample. All electrical measurements were done on the Keithley SCS-4200 Parameter Analyser (PA), with a noise ceiling of $500 \mathrm{pA}$. Unless otherwise noted, the gate of the device was grounded (at $0 \mathrm{~V}$ ) and not left floating. Optical density was measured with a $405 \mathrm{~nm}$ Thorlabs laser diode.

PA SMU Voltage Application The results below frequently make reference to measurement time and use variation in the voltage application time in order to change parameters. Due to this it is important that the 
internal methodology of how the PA applies voltage to the non-grounded contact are understood. When measuring current the SMU is measuring the current that is being sunk at the contact that is being held at some voltage, relative to the SMU ground. All of these measurements were done with the inactive contact held at that same ground level. The SMU then applies a voltage for a series of times values indirectly defined by the input measurement parameters. This is shown for a voltage sweep in Figure 4.1. Hold Time (HT), Delay (D) all Sweep Delay (SD) increase in duration

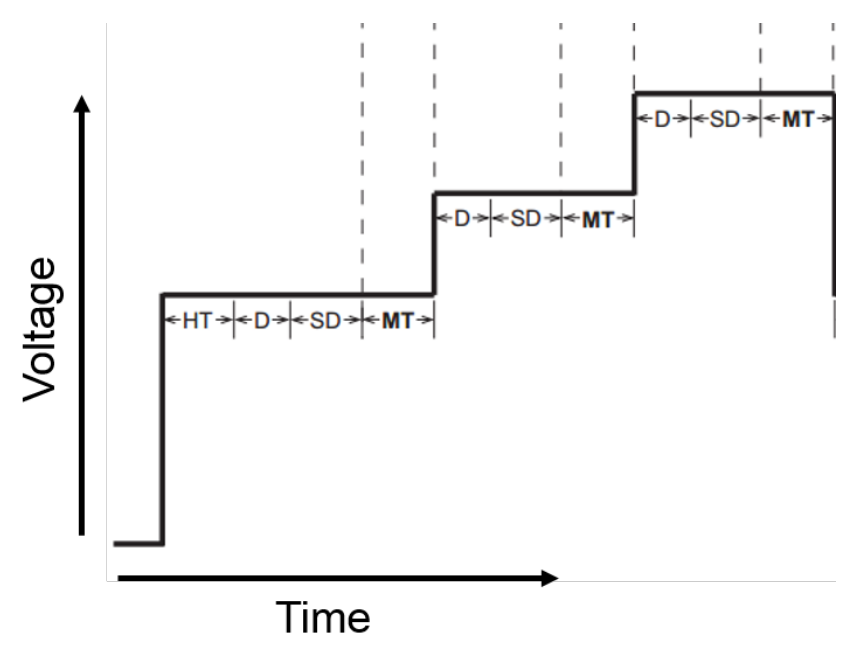

Figure 4.1: Application of voltage over time during measurement by SMU in the PA system. Reproduced in part from the Keithley Model 4200-SCS Reference Manual, 4200901-01, Feb 13, page 6-143.

for slower measurements, this means that the device is being held at each measurement value voltage for a longer time. Measure Time (MT) is unaffected by the desired measurement speed but is affected by the magnitude of the signal, where lower currents will have longer measure times. Experimentally, this means that during slower measurement voltage sweeps the voltage has been applied to the device for a longer time. For devices with a time independent resistance this has no second order effects. However, for devices that have some degree of memory of charge history then the measurement speed would correlate to the amount of charge that has 
passed into the material before the instantaneous current is measured.

\subsection{Results}

Although the initial hysteresis curves in this section were observed with various HgTe QD sizes on various devices, all final measurements of the memory effect and its related behaviour were obtained with the QD batch shown below.

\subsubsection{Initial Characterisation}

The QDs used in the best performing memory devices were characterised optically (as EDT treated films) and with TEM. Figure 4.2 shows a histogram of the sizing overlaid over a TEM image of the QDs used. Using

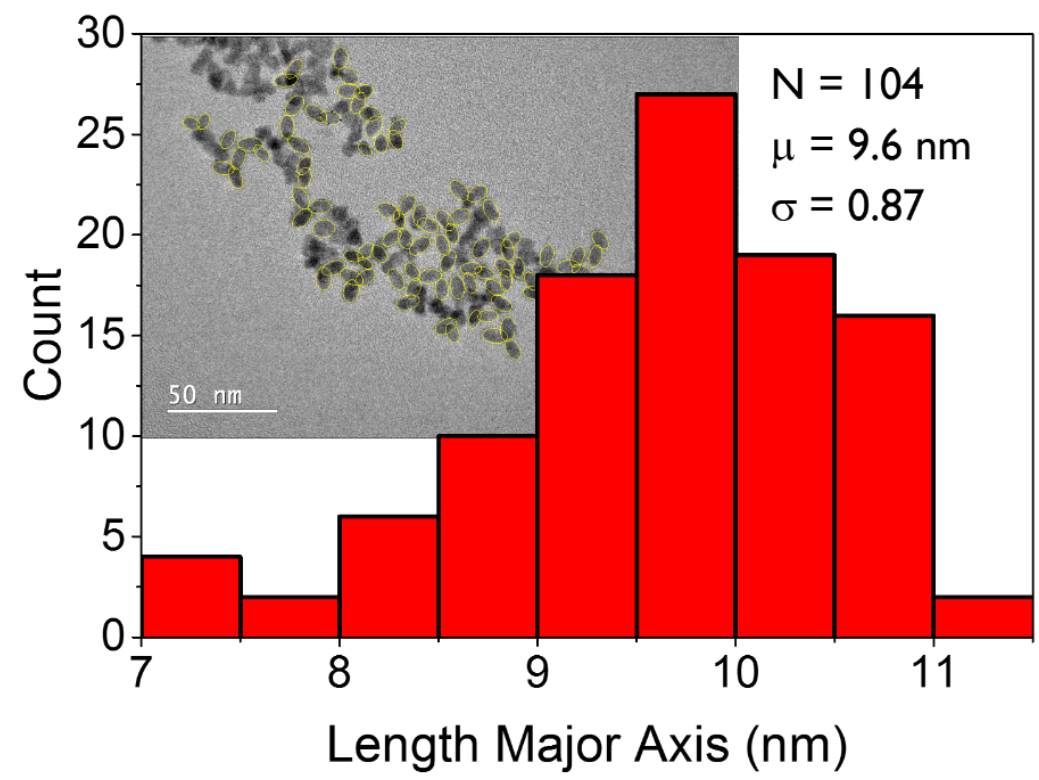

Figure 4.2: TEM image and particle sizinfor the QDs used in the devices reported and analysed in this Chapter. The yellow circles are a legacy of the sizing tool used to give the major axis length. 
the published empirical relationship between particle size and HgTe QD radius [100],

$$
\lambda_{E_{g}}^{Q D}=\frac{30.5}{\sqrt{\left(1+\frac{43}{R_{Q D}}\right)^{2}}-1} .
$$

It is found that the calculated bandgap from the mean major axis length is $3.8 \mu \mathrm{m} / 0.326 \mathrm{eV}$. This is corroborated by a Tauc plot of a spin coated, EDT treated film on a $\mathrm{CaF}_{2}$ substrate. This is shown in Figure 4.3 and estimates the bandgap of the film at $0.324 \mathrm{eV}$, so both methods are in good agreement.

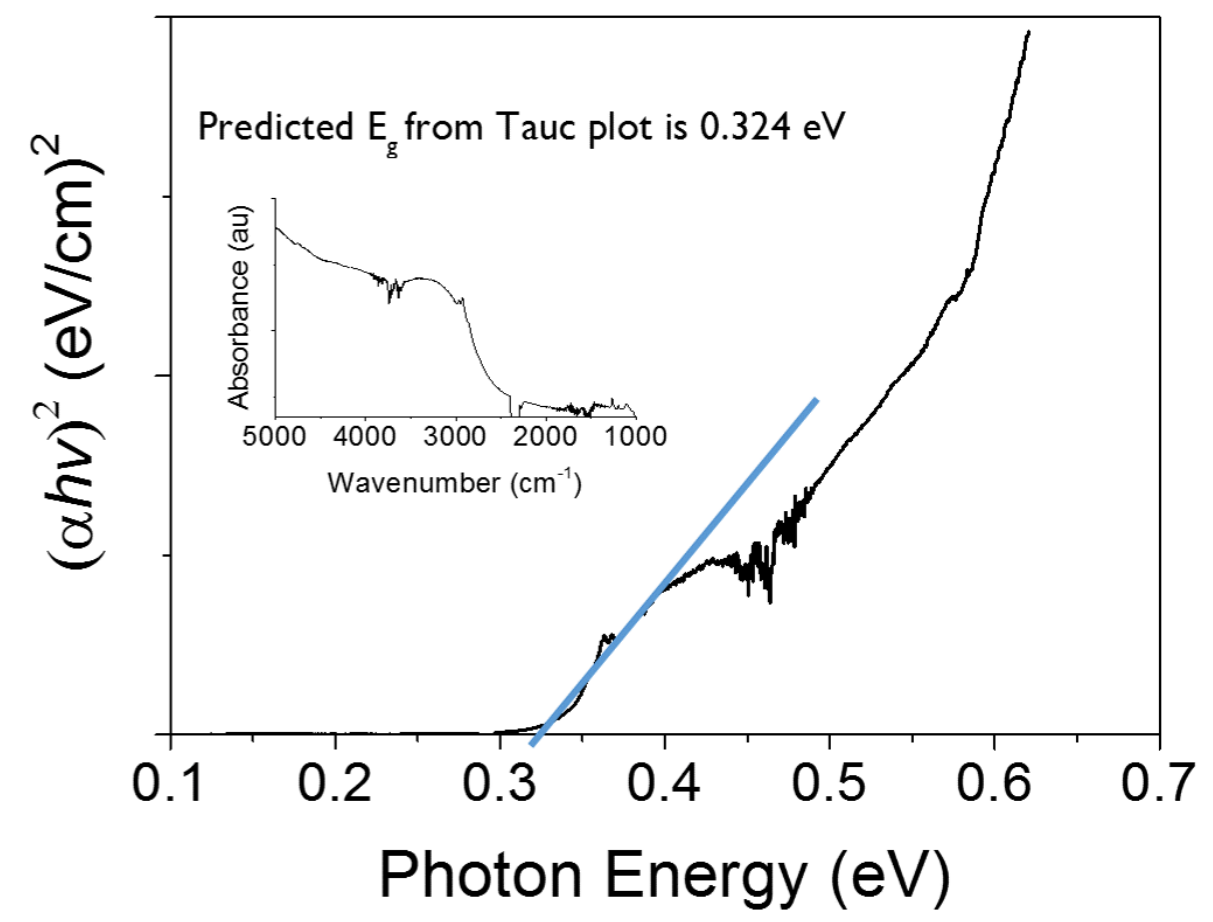

Figure 4.3: Tauc plot (direct allowed transition $r=1 / 2$ ) of the film absorbance shown in the inset. The blue line represents the slope fit that gives the annotated bandgap value.

SEM images were taken of the top/cross section of the devices using carbon coating and a stub that had an off vertical mount. Figure 4.4 shows 
that the device has the expected construction, the most interesting aspect being the relatively low thickness of the final film, measuring $30 \mathrm{~nm}$. The edge feature of the gold is an expected result of using single step positive resist without an undercut in the resist profile. EDS mapping of the same top/cross section area of the device as in Figure 4.4 is shown in Figure 4.5. Several points should be noted when looking at Figure 4.5:

1. There is a line overlap between $\mathrm{Au}$ and $\mathrm{Hg}$ therefore the even film coverage is best indicated by the Te map.

2. The oxygen plot is not evidence of oxygen adsorption into the film due to the $\mathrm{SiO}_{2}$ layer beneath providing a huge oxygen background underneath the film.

3. The scattering of aluminium electrons from the SEM mounting stub is included as it provides a topographical image of the film surface.

4. There were no other detectable elemental peaks in the EDS film analysis, indicating a lack of any major impurities.

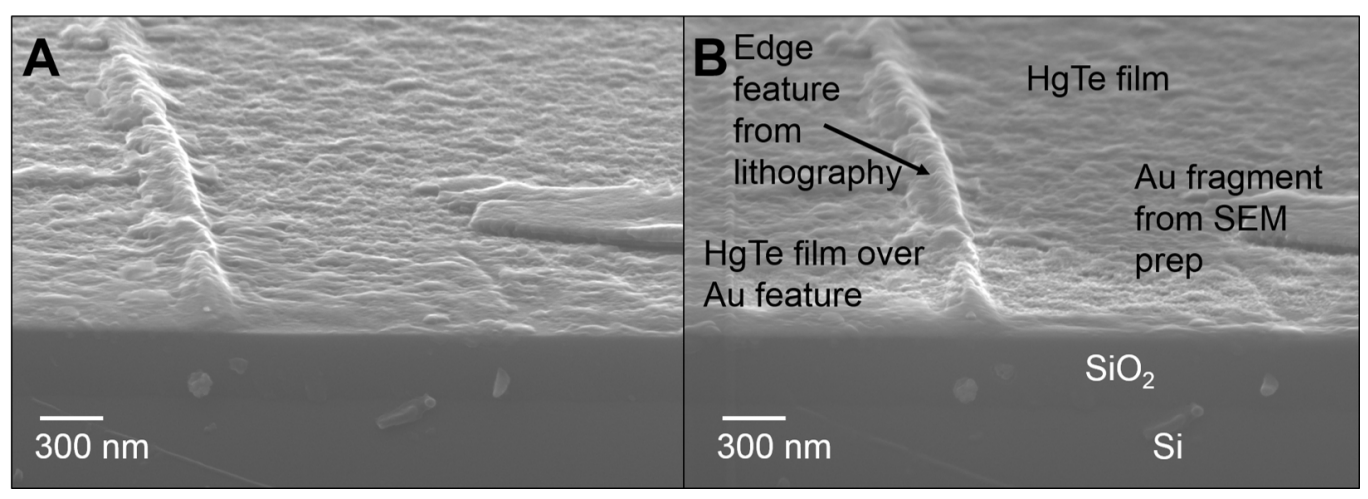

Figure 4.4: A, SEM SEI image at X40K of a device cross section and film surface, mid plane focus. B, SEM SEI image of the same area as $A$, annotated for ease. Focus here is on the film nearest the front edge. From these images the film thickness is determined to be $30 \pm 10 \mathrm{~nm}$ 


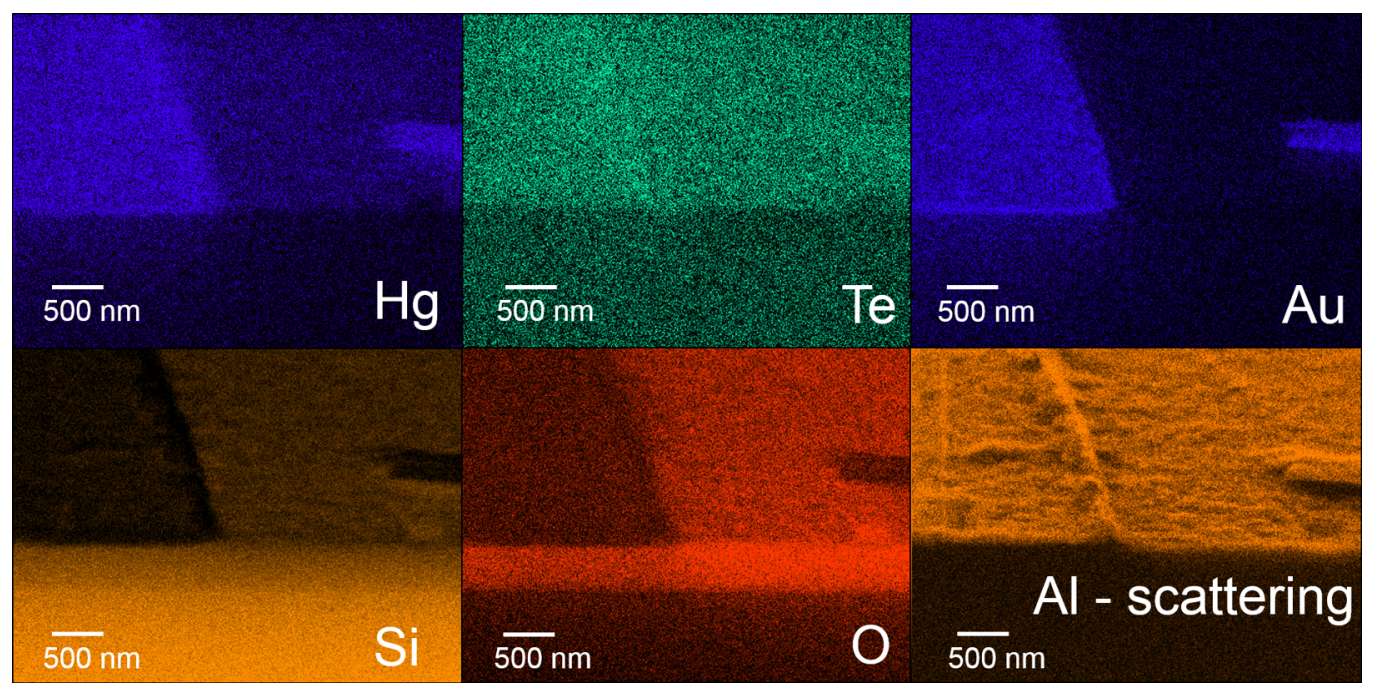

Figure 4.5: SEM EDS mapping of the same area as in Figure 4.4, discussed in the text.

Another parameter often used when discussing NC films is the packing density, which defines the percentage of the film volume that is NC rather than ligand, empty space or impurity. As the particle size and film thickness is known, the packing density can be estimated from optical measurements. Using published information on the optical cross section of $\mathrm{Hg}$ atoms to $415 \mathrm{~nm}$ light [100] we can calculate the packing density (P) of our film with Equation 4.2. $405 \mathrm{~nm}$ light was used in the referenced test for optical depth however there should be little variation between $405 \mathrm{~nm}$ and $415 \mathrm{~nm}$ behaviour.

$$
P=V_{Q D}[Q D]=\frac{a^{3}}{4} \ln 10 \cdot\left(\frac{O D}{\sigma_{H g} L}\right)
$$

The prefactor on the right-hand side of Equation 4.2 defines the packing density as the product of the volume of a single QD and the concentration of those QDs in the volume of interest. This is then further defined where $a$ is the HgTe lattice parameter $\left(6.46 \times 10^{-10} \mathrm{~m}\right), 4$ is the number of $\mathrm{Hg}$ atoms per unit cell, $O D$ is the optical depth, $L$ is the path length (film thickness $=30 \mathrm{~nm}$ ) and $\sigma_{H g}$ is the absorption cross section of $\mathrm{Hg}^{2+}$ at 415 
nm. For our films and after accounting for any losses through the $\mathrm{CaF}_{2}$ substrate the optical depth was measured to be 0.202 at $405 \mathrm{~nm}$, predicting a packing density of 0.4 . This can be checked for validity by looking at the absorption coefficient of the film relative to bulk HgTe. For $0.4 \mathrm{eV}$ and using published data bulk HgTe has a lower bound for its absorption coefficient at $14 \times 10^{3} \mathrm{~cm}^{-1}[184,185]$. Taking the absorption of the film at 0.4 $\mathrm{eV}$ we get an absorption coefficient of $7.6 \times 10^{3} \mathrm{~cm}^{-1}$. The upper bound of the packing factor for our films, i.e. assuming no reflection and all absorption in the HgTe is thus 0.54 . These two methods combined suggest that the packing density of the films lies between 0.4 and 0.55 .

\subsubsection{Hysteresis}

When measuring the IV curves of these devices after days of air exposure there was a large degree of repeatable and reproducible hysteresis. This is shown in its simplest form in Figure 4.6. The devices showed different current flow when voltage (applied field) was increasing to that displayed when it was decreasing.

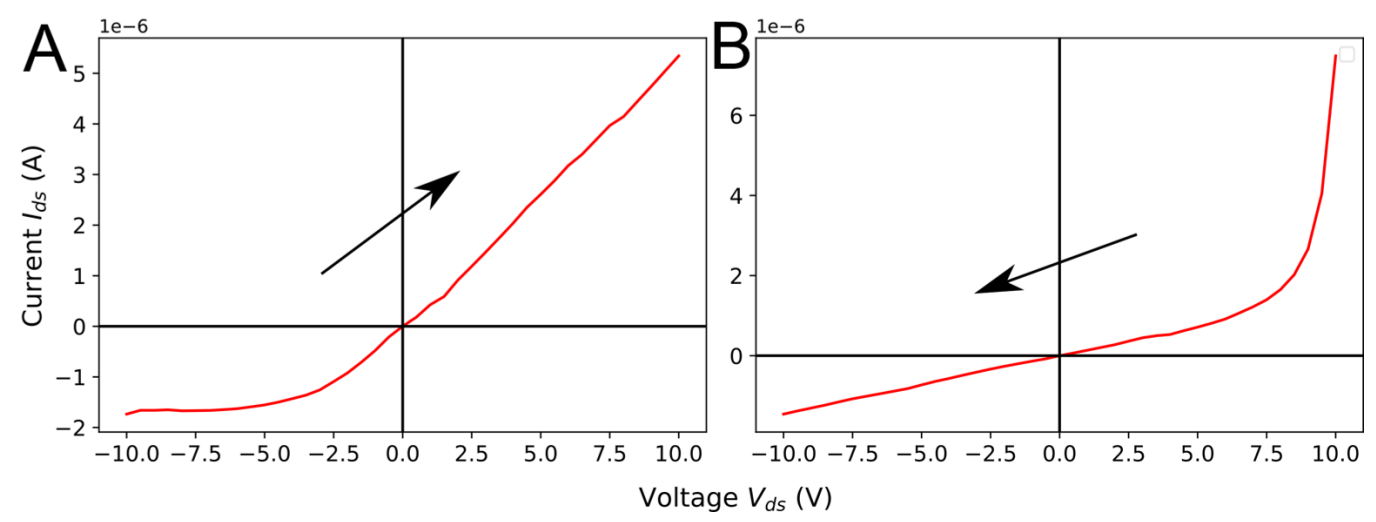

Figure 4.6: $A, I V$ sweep from $-10 \mathrm{~V}$ to $+10 \mathrm{~V}$. B, IV sweep from $+10 \mathrm{~V}$ to $-10 \mathrm{~V}$. The black arrows are showing the voltage sweep direction. The current behaviour varies strongly with sweep direction, it is worth restaing here that the noise of the system is at least 4 decades lower than the signal.

This behaviour is shown in more detail in Figure 4.7. From this Fig- 
ure it can firstly be seen that the hysteresis is real as it is independent of the measurement condition. For the positive quadrant it can be seen that the behaviour under increasing voltage (solid line in Figure $4.7 \mathrm{~A}$, dashed line in B) is independent of start voltage, as all behaviour under increasing voltage fits to the same line. Contrary to this is the behaviour under decreasing applied voltage, where the shape of the curve is defined by the start voltage, and then explained by some decay from that initial measurement point. These statements are reversed when considering the negative quadrant, where the measured currents are considerably lower but still measurable. As is shown below these films behave as ambipolar transistors, but with a higher hole mobility than electron mobility. The inverted quadrant response seen here coupled with the known p-type behaviour means that the material is reacting differently to hole injection/electron withdrawal (positive voltage) than electron injection/hole withdrawal (negative voltage).

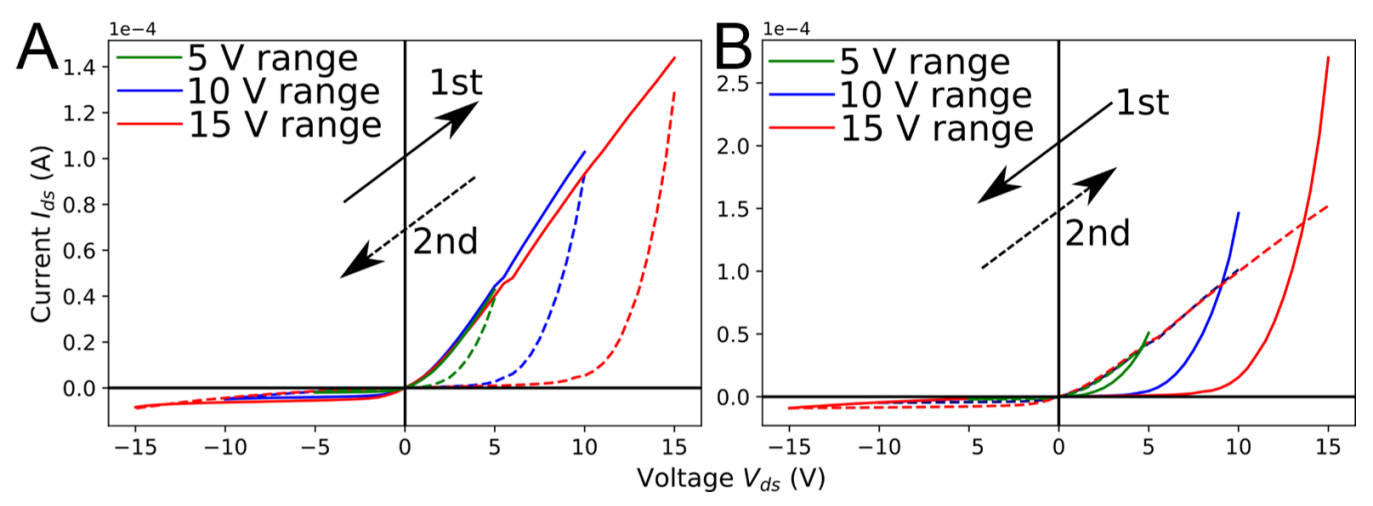

Figure 4.7: $A, I V$ sweeps with various start voltages that begin at -ve V. B, IV sweeps with various start voltages that begin at $+v e \mathrm{~V}$. The arrows show the sweep direction for the corresponding line type, the colours define the start and finish voltage values for that sweep. The text discusses this behaviour with reference to the positive quadrant.

Continued investigation of the basic IV curve by varying measurement time parameters, i.e. increasing or reducing the charge flow into the material, had a major effect on the shape of the hysteresis. This is shown 
in detail in Figure 4.8 for three measurement speeds, and the entire hysteresis loop is shown for the extremes of measurement in Figure 4.9. The

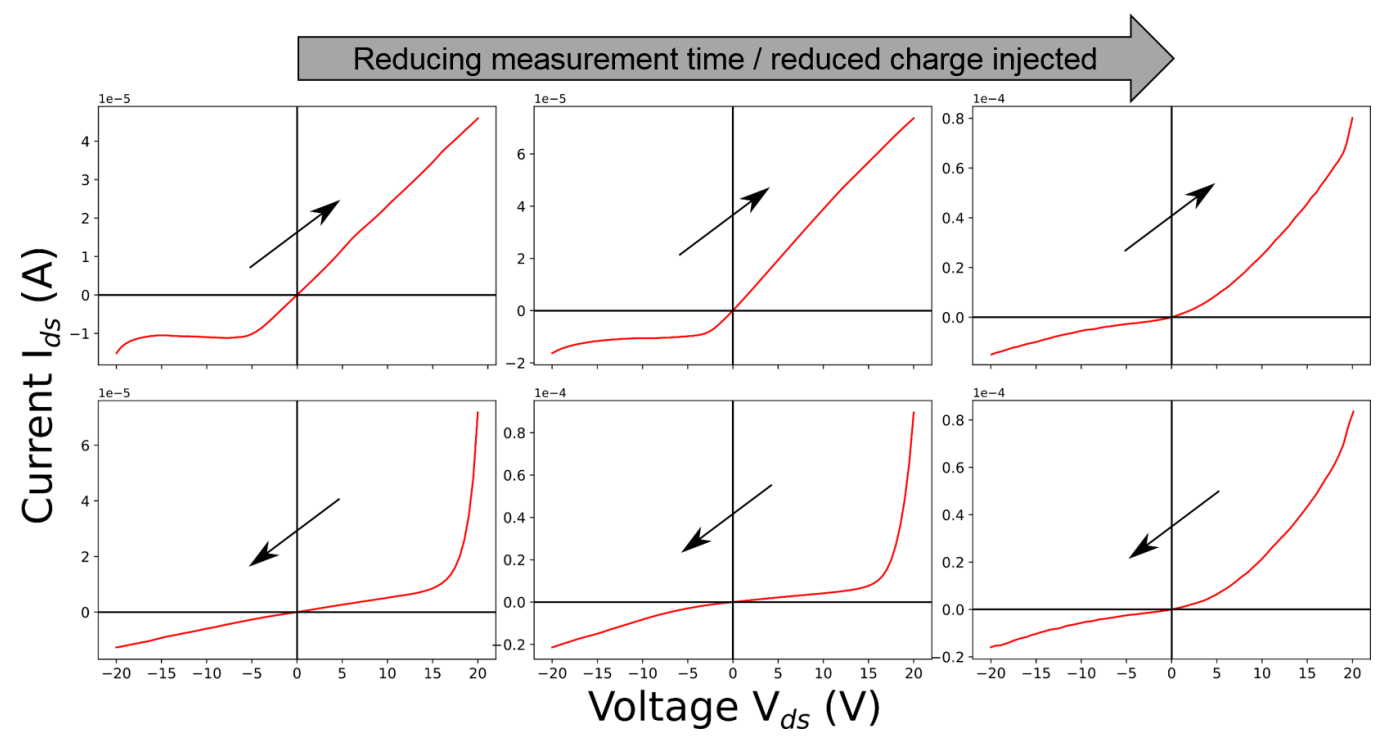

Figure 4.8: IV traces for increasing measurement speed (left to right) for increasing applied voltage (top) and decreasing applied voltage (bottom). As the measurement time decreases the behaviours under different sweep direction tend towards a similar behaviour, this suggests that the measurement time, and hence the charge flow during it, is in some way responsible for the hysteresis.

fastest measurement shown in Figure 4.9 is actually a fast measurement with an added settle time (of zero applied voltage) before the measurement is applied. It can be seen in Figure 4.9 that the hysteresis is considerably reduced by allowing a settle time. This variation of behaviour with measurement time implies that there is a time dependent resistance to the material that is also sensitive to carrier type. These observations show that these devices exhibit an implicit memory of charge flow and therefore memristance. 


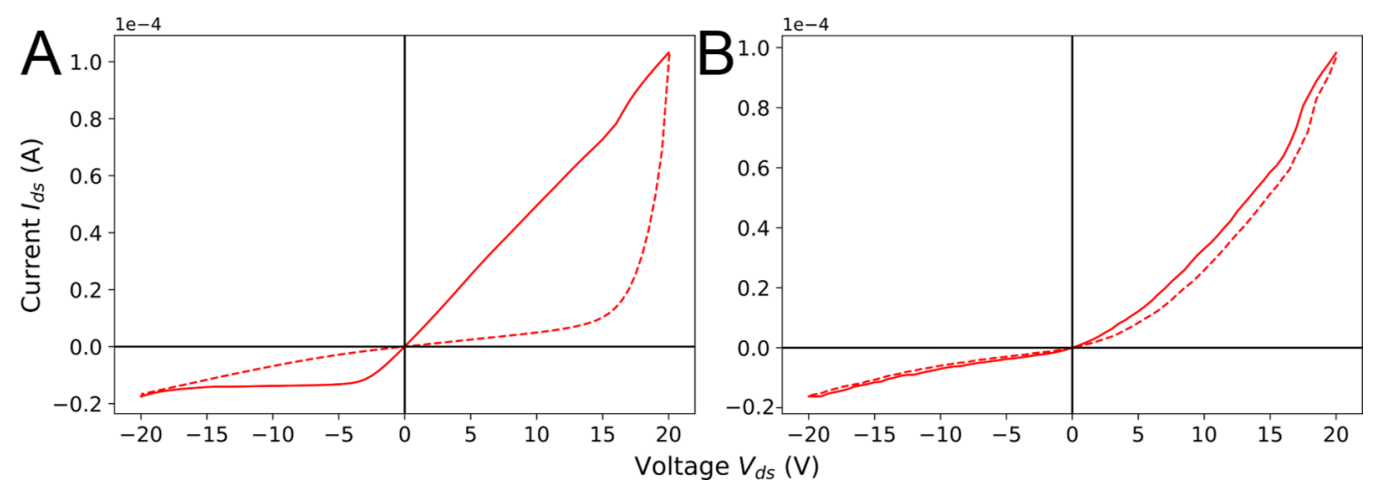

Figure 4.9: A, Major IV hysteresis seen with slow measurement. B, Minor IV hysteresis seen with fast measurement and settle time. This clarifies the left tor right trend seen in Figure 4.8.

\subsubsection{Memristance and the Memory Cycle}

Memristive devices are electrical components that have a resistance that is dependent upon some changing state in the material. Since the IV curves suggest that the degree of charge flow directly affects the resistance, it will be assumed that the state that resistance is dependent upon is previous charge flow. Memristive behaviour, as opposed to bi-stable switching, sets the system in a state that is directly dependent upon the quantity of previous charge flow and not merely the crossing of a particular threshold value. Memristance here is defined by the state dependent Ohm's law (Equation 4.3) and the state equation (Equation 4.4) [150,153].

$$
\begin{gathered}
i=G(x) \cdot V, \\
\frac{\delta x}{\delta t}=g(x, V),
\end{gathered}
$$

where $i$ is the current, $V$ is the applied voltage (from the read step), $x$ represents one or more state variables which are internal parameters that are independent of the present applied voltage. $G(x)$ is the memductance, the inverse of the electrical memristance and is analogous to the conduc- 
tance of a conventional ohmic system. $G(x)$ is used to differentiate between a current, charge or voltage controlled system where the parameter that controls behaviour is $x$ and the relationship between the measured current and $x$ is then $G(x)$ [150]. The change in the internal state over time is commonly represented as some function $(g(x, V))$ of the internal state $x$, and the applied voltage, $V$ during the write and erase pulses. These equations suggest that applied voltage changes the charge flow behaviour and that the current at any point is related to some function of the charge history.

Practically speaking, at high absolute voltages (e.g. the read and write steps) detectable internal state changes occur, while at low applied voltages (e.g. the read step), the current can be measured with minimal alteration to the internal state. Thus we can design a protocol with the aim of using the charge memory of the system to provide electrical memory that is further modulated by light and gate doping. The steps of the memory cycle being i) the erase step, positive voltage to write a " 0 ", ii) the read step, $0.1 \mathrm{~V}$, iii) the write step, negative applied voltage. The on/off ratio is calculated as the ratio of the measured current in the read step following the write step, to that following an erase step. It is a single value that encapsulates the ratio of the HRS to the LRS, the relationship between the hysteresis and the HRS/LRS is shown visually in Figure 4.10. 


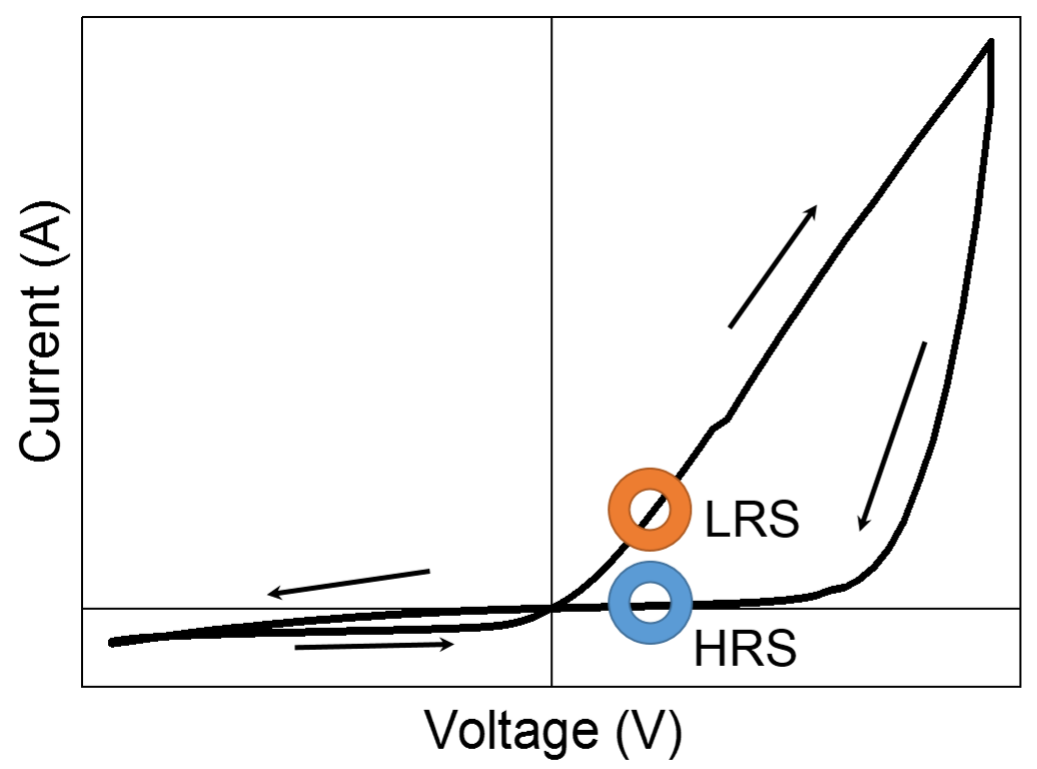

Figure 4.10: IV curve showing the position of the HRS and LRS probed by the read states on the IV curve. The black arrows are showing voltage sweep direction, on/off can be thought as HRS/LRS measured at $0.1 \mathrm{~V}$, as this is the read voltage used throughout the rest of this chapter.

\subsubsection{Time Evolution}

As the hysteretic behaviour detailed above was far less prominent in newly made devices, the time evolution of the hysteresis should be analysed. This can be most clearly examined with the use of the on/off value as a proxy for how pronounced the hysteresis is. Understanding the factors that drive the development of the memory/hysteresis effect should allow a more complete understanding from a material perspective and following from that, the correct mechanistic viewpoint. Air exposure will form p-type solids in many II-VI and IV-VI NCs as oxygen that is adsorbed onto the NC surface is expected to create traps [186]. The behaviour of the devices during exposure to oxygen (in the form of ambient air) was tested. Figure 4.11 A shows how the on/off value of the devices increases with time in air. This indicates that either $\mathrm{O}_{2}$ or $\mathrm{H}_{2} \mathrm{O}$ 
are responsible for the changing behaviour. Oxygen is proved to be the
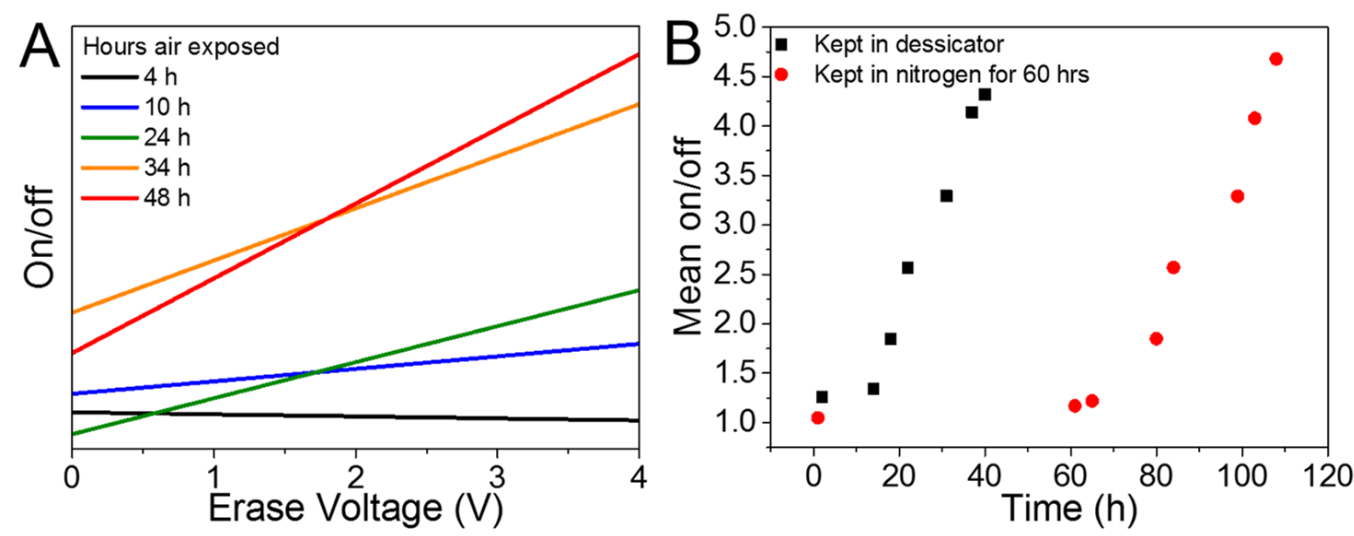

Figure 4.11: $A$, shows the fit lines (from 160 data points per line) for the on/off values for different erase voltage values, measured at various times after fabrication. Note that the on/off value also increases with the erase voltage, this behaviour is discussed fully in the rest of this Chapter. B, shows the on/off evolution of two devices made concurrently but stored under different conditions for the first $60 \mathrm{~h}$. The nitrogen stored device only starts to develop memory after being exposed to air.

most likely cause by Figure 4.11 B, which shows the behaviour of two identical devices fabricated concurrently in air. One device is fabricated and left in a desiccator (not vacuum, humidity 20\%) and removed when required to measure the memory effect. The other device was fabricated and then placed in a nitrogen glovebox for $60 \mathrm{~h}$, and once removed it was left in air in-between measurements. It can be seen that there is very little increase in measurable on/off after $60 \mathrm{~h}$ in nitrogen. Once removed the onset of the effect occurs at a similar rate to the desiccator stored device. As the moisture content in the air was different for the two devices during the progression this subtley points to atmospheric oxygen rather than atmospheric water being responsible.

If oxygen is being adsorbed into the film, and onto the surface of the dots creating trap states, this should be measurable directly. Rather than as inference from indirect measurements as seen above. To investigate this 
two identical samples of QDs were dispersed onto TEM grids. One was stored in a non-vacuum desiccator and the other was stored in a nitrogen glovebox. The samples were then looked at with EDS in the TEM. These samples were not oxygen plasma cleaned prior to imaging. Due to

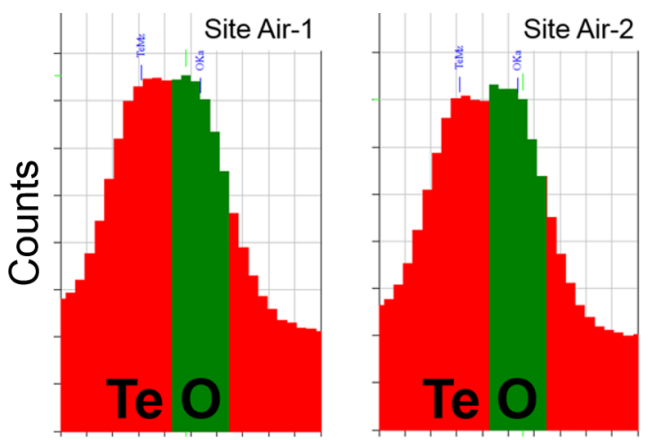

Air stored sample
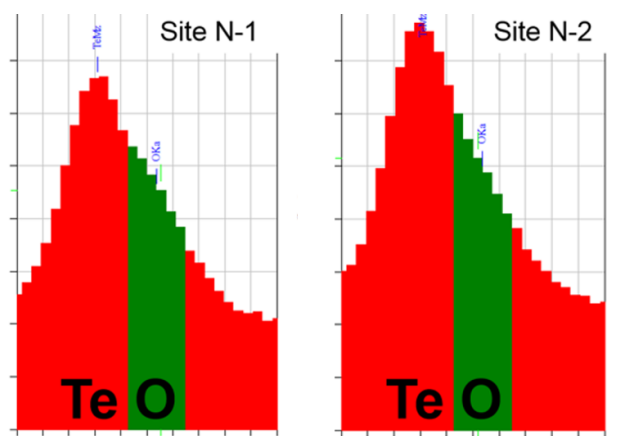

Nitrogen stored sample

Figure 4.12: EDS spectra for the main $O$ line and a secondary Te line. A, spectra for two sites on the desiccator stored sample. B, spectra for two sites on the nitrogen stored sample. The key result is given by the ratio of tellurium, which is the same between samples, to oxygen.

the presence of the tellurium line this data shows us that for two identical QD samples the relative amount of oxygen to tellurium substantially decreases when the sample is not stored in an oxygen filled environment. The oxygen is adsorbing onto exposed sites on the QDs and causes a trap state that leads to charge history in the device. Due to the p-type nature of the material, the larger hole mobility and much greater current change in the positive quadrant of the IV curve both indicate that the traps are hole traps and the movement of holes through the material, and into and out of the active electrode for negative and positive voltages respectively, is responsible for the behaviour.

\subsubsection{Transistor Behaviour}

Concurrently to the evolution of the hysteretic behaviour it was also observed that the devices developed robust transistor characteristics. Prior 
to this HgTe NC thin film transistors (TFTs) had been shown not to display any gateable behaviour unless cooled [99], or sintered [187,188]. Sintering destroys the bandgap arising from confinement in the NCs. As expected the freshly made devices did not show any obvious gating behaviour, however after $48 \mathrm{~h}$ exposure in air the devices demonstrated perfect ambipolar behaviour. That is to say the same device demonstrates both p-channel enhancement and n-channel enhancement with no change in structure or electrode material [189]. The key identifying feature of ambipolar devices is that the the output characteristics show three clear regimes, linear, saturation and superlinear/breakdown. Both the $\mathrm{n}$ - and p-channel output curves for the same device are shown in Figure 4.13.
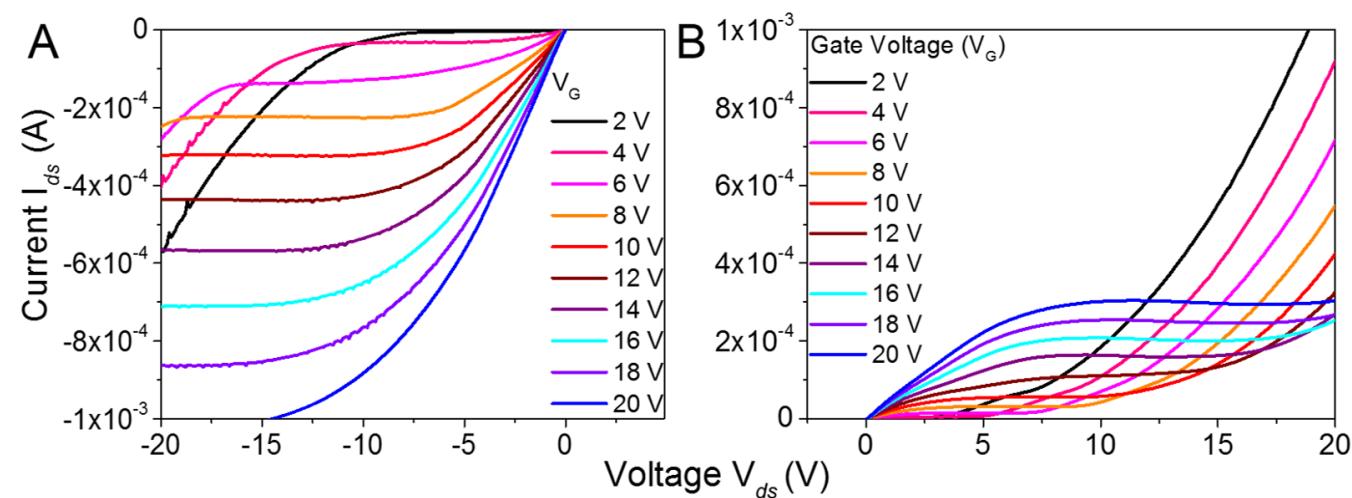

Figure 4.13: A, p-type output. B, n-type output. Both of these curves are taken for the same device under the same conditions, this device is demonstrating clear ambipolar behaviour.

The existence for these regimes was covered in Chapter 1. In short they provide definitive evidence that both carriers exist in the material, and more importantly that both can be injected or extracted at the electrodes. The mobility of the carriers can be determined using Equation 4.5, valid in the saturation regime [19].

$$
I_{D}=\frac{\mu C W}{2 L}\left(V_{G}-V_{T}\right)^{2}
$$


This calculation requires a threshold voltage value $\left(V_{T}\right)$. For ambipolar devices the threshold voltage is most easily found by looking at the saddle point of the transfer curve [190], although other methods such as plotting $\sqrt{I_{d s}}$ against $V_{G}$ and extrapolating to the x-axis intercept are also commonly used [190]. However, there are several general issues that should be highlighted about this process. Firstly, in materials that exhibit the bias stress effect it is vital that transfer curves are measured rapidly to avoid the slow screening of the gate field, as was first highlighted and quantified by Liu et al. in 2010 [176]. This is demonstrated in our devices in Figure 4.14 $\mathrm{B}$ where the $\mathrm{x}$-axis position of the saddle point is dependent upon sweep direction and timing. Thus the median minimum $x$-axis position is taken as $V_{T}$. Secondly, the position of $V_{T}$ is dependent upon $V_{d s}$ (Figure $4.14 \mathrm{~A}$ ) yet the saturation regime exists for a span of $V_{d s}$ values, i.e. there are a set of mobility values that are equally valid. Due to this the mobility values quoted below are for $V_{d s}$ values that correspond specifically to the onset of the saturation regime, for a given $V_{G}$. This gives only a single value for mobility, rather than a span. Clearly if more detailed work were to be carried out on these transistor characteristics then this would have to be resolved. However, here, these results are only used to provide evidence that there are two mobile carriers, holes are more mobile than electrons, and that the material is gateable.

As these ambipolar characteristics develop in oxygen it is entirely possible that the best way to model this system would be to use a band bending model applied at the surface region of each quantum dot. Ambipolar behaviour has been observed before in small bandgap nanowire transistors [191] and is attributed to surface layers causing a p-type layer in contrast to the n-type dominant bulk, or vice versa. Clearly the ligands in the system and the separation of the QDs represent a conceptual barrier, however in this system it is entirely possible that the adsorption of oxygen causes upward band bending at the ligand-QD surface interface. This 
could lead to a p-type enhancement layer at the surface if the valence band is bent above the fermi level. To extend this hypothesis it is then suggested that the reduced mobility of electrons is due to the now increased physical separation between core regions as the effective surface region increases the required electron hopping distance. The calculated mobilities for these

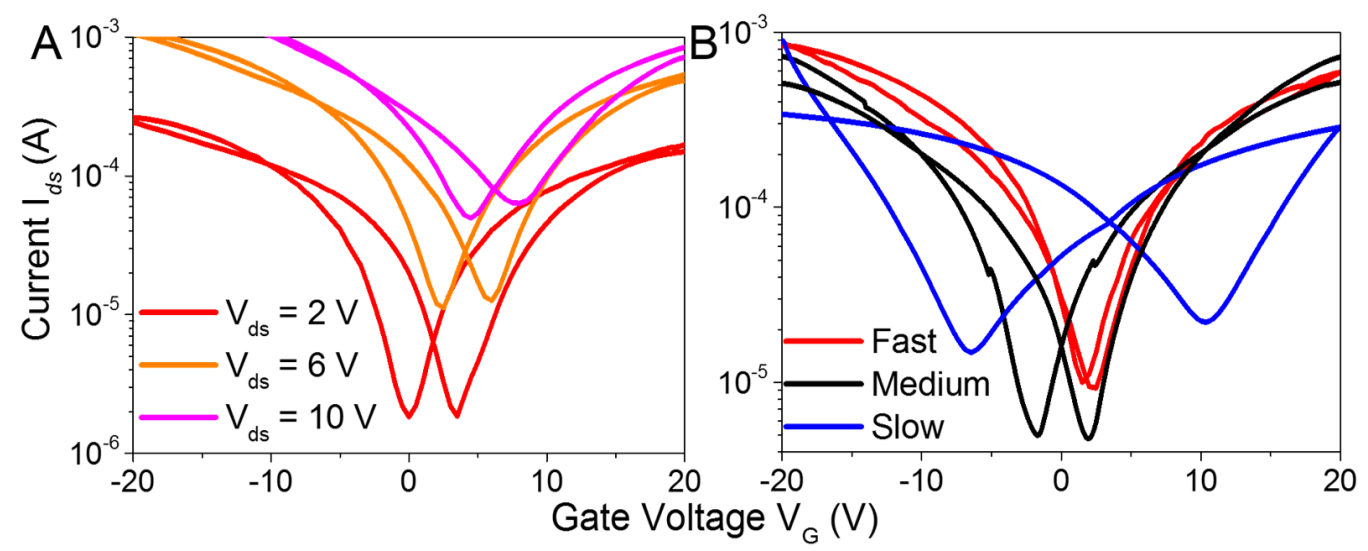

Figure 4.14: $A, V_{T}$ varying with $V_{d s}$, showing that stating a threshold voltage to calculate a mobility without stating the drain voltage is ambiguous in these devices. B, Transfer sweep crossing with sweep speed, $V_{d s}$ is held at $5 \mathrm{~V}$ throughout.

devices were $\mu_{e}=0.021 \mathrm{~cm}^{2} \mathrm{~V}^{-1} \mathrm{~s}^{-1}$ and $\mu_{h}=0.083 \mathrm{~cm}^{2} \mathrm{~V}^{-1} \mathrm{~s}^{-1}$. These values fall within the range expected for hopping conduction [192], which here appears to be dominated by holes, and are lower, as expected, than those reported for sintered HgTe NCs [187]. However, they are higher than those previously reported for non-sintered HgTe NC films, with a proportionally higher two terminal conductivity obtained here using a far thinner silicon oxide layer [99].

\subsubsection{Memory}

The following results are the key memory results used for defining the metrics of the device and for explaining the mechanisms at work in the system. They will be referred to throughout the Discussion Section of this Chapter. One way to describe this device is that it has a High Resistance 
State (HRS) that is caused by application of a large ( $>1 \mathrm{~V}$, erase) positive voltage. It also has a Low Resistance State (LRS) that is caused by application of a large negative voltage (write). To detect the state a low positive voltage (read) is used to probe the device, Figure 4.10 shows this visually. Due to the memristive behaviour there is a current difference (on/off ratio) between these two states and it is related to the previous charge flow.

The highest on/off ratios obtained with these devices are shown in Figure 4.15. The tuning of the light conditions to give the highest on/off ratios will be discussed later in this Chapter, but it can be seen that these devices have reliable and reproducible on/off response over a time period of days, and that the on/off value is always greater than 10 . The potential of the devices in terms of raw on/off values are demonstrated by Figure $4.15 \mathrm{C}$ which shows a run in which the median on/off was 50. 

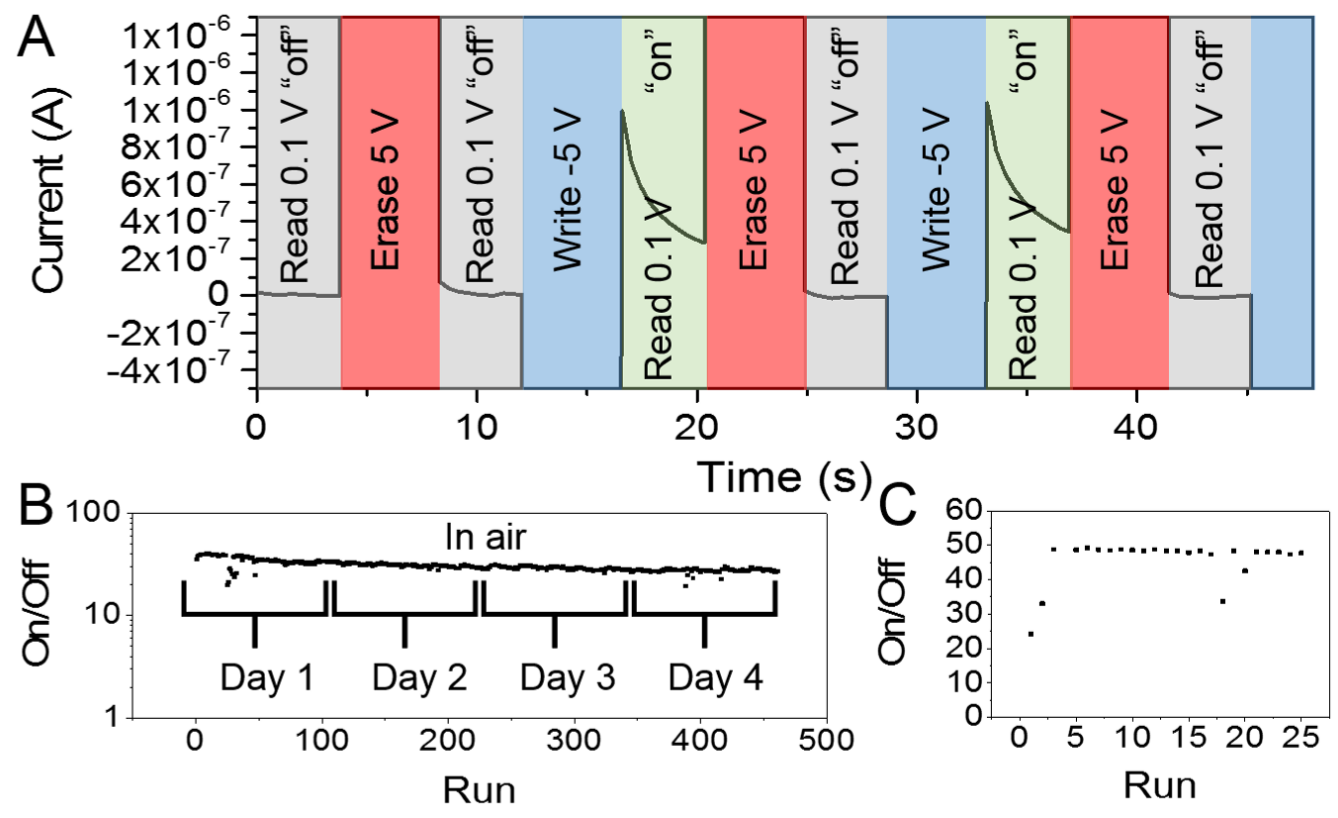

Figure 4.15: A, the memory cycle overlaid over a typical output signal, the black line indicates the current. This is for a device with the incident light intensity tuned to give a detectable on/off but a minimum decay of the off state. B, on/off ratio of best performing device over 450 memory cycles, with groups of runs spread evenly across a $96 \mathrm{~h}$ period. For the on/off shown the device was using an absolute write/erase voltage of $7.5 \mathrm{~V}$. C) The 25 cycle run with the highest average measured on/off ratio. Copyright ACS Appl. Mat. E Int.

To prove that the observed memory cycle and its behaviour are purely device related and independent of measurement the memory cycle was performed with long read periods. The read period was then pulsed between $0.1 \mathrm{~V}$ and $0 \mathrm{~V}$. The results are shown in Figure 4.16 where it can be seen that the current at any time in the read period is independent of, and not perturbed by, the measurement method. 


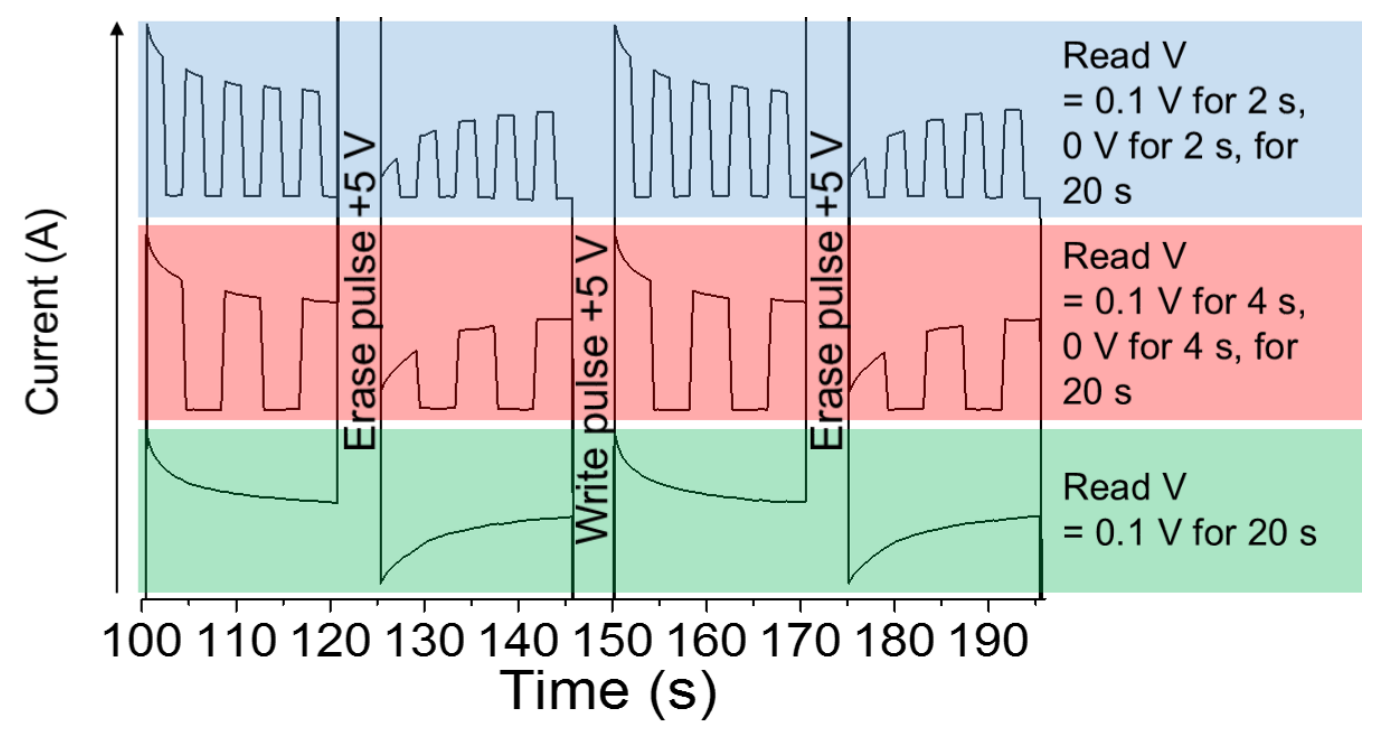

Figure 4.16: Different measurement protocols (blue=2s@0.1 V/0 V, red = $4 \mathrm{~s} @$ $0.1 \mathrm{~V} / 0 \mathrm{~V}$, green = $20 \mathrm{~s} @ 0.1 \mathrm{~V})$ y-separated, with synchronised write/erase pulses. The decay over time in the current measured during the read step is independent of the measurement voltages, therefore it is a characteristic of the material.

In terms of other commonly quoted memory metrics [193] this device has a read time that was reliable down to $10 \mathrm{~ms}$. The retention time for the on state, for the best performing devices, had an on/off ratio $>10$ for the first $6 \mathrm{~s}$ following the write step and the on/off ratio remained $>2$ for $10 \mathrm{~s}$. As DRAM is refreshed every $64 \mathrm{~ms}$, having a retention time that is longer than a second is of great interest and warrants further investigation. It should be noted that, by definition, the write/erase time in a true memristive system is arbitrary; a longer time or a higher voltage (leading to a higher current and thus more charge movement) will give a higher on/off. This can be shown explicitly by calculating the charge that has been measured moving during the erase step, by integration of the current vs time plot. Various devices were measured with erase voltages between 0.1 and $10 \mathrm{~V}$ and erase times between 0.5 and $20 \mathrm{~s}$. For this range of charge values the on/off value was recorded, normalised for each device and plotted in Figure 4.17. There is a linear relationship between on/off and charge 
up until some saturation of on/off. This transition represents the point at which the trap filling region reaches some threshold population following the write/erase steps.

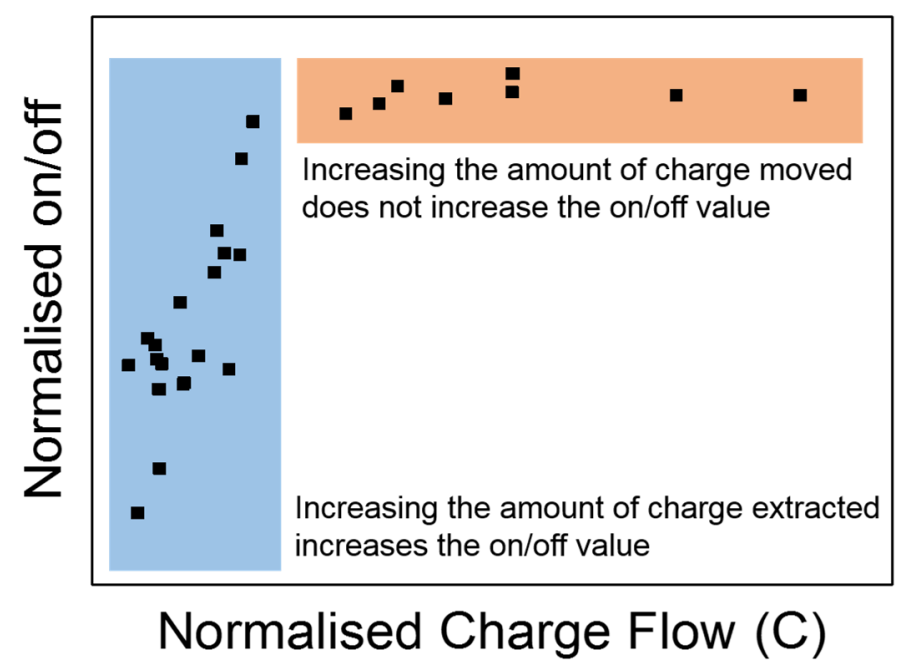

Figure 4.17: The change in mean on/off with charge movement in the erase step, normalised per device and shown for multiple devices. This is to show that multiple devices all have the same trend of behaviour, although the measured values are different.

The other key behaviour of the device is the effect of illumination on the device, this will be discussed in more detail in the next section. However, to begin it should first be noted that the increase of carriers that occurs in the material when illuminated acts to turn the memory of the system on and off. This is shown in Figure $4.18 \mathrm{~A}$, there is no detectable memory effect in the dark but an obvious effect when illuminated.

Since these devices can be effectively doped with free carriers they were operated with various gate voltages in an attempt to create electrically gated memristors, the on/off state currents change as would be expected. This is shown in Figure 4.18 B, with negative (positive) gate voltages enhancing (suppressing) the p-type mobility. On closer inspection the off 

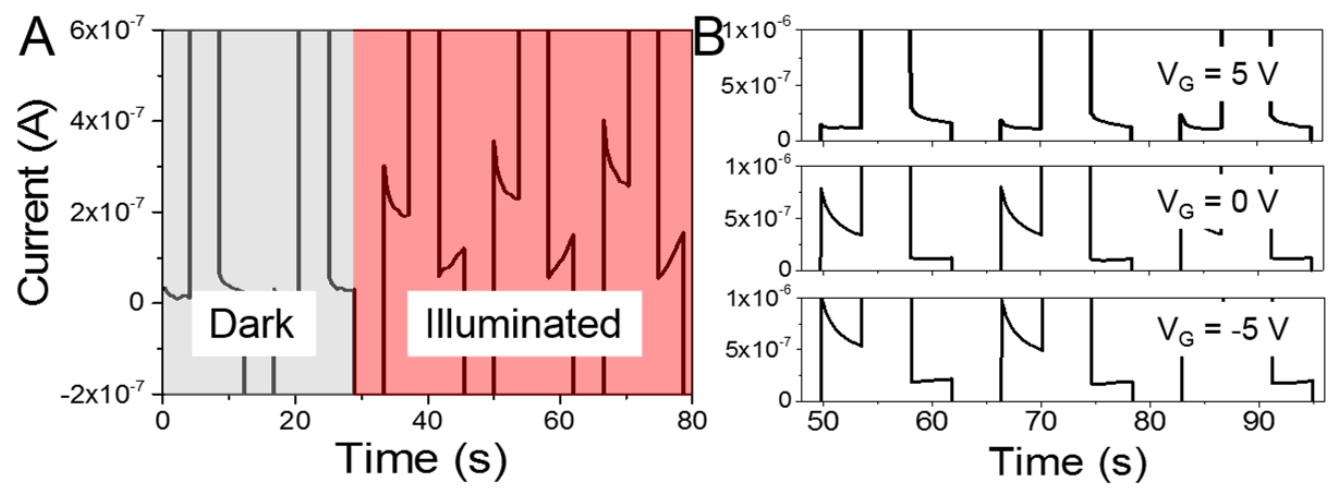

Figure 4.18: $A$, light activated memory. The slow photoresponse is evident in the current increase of the on state during illumination. B, time matched output signal of the same film under 3 different gate biases, the y-axis is kept the same for illustrative purposes.

state behaviour can also be seen to vary. At positive gate voltages the off state shows a negative decay not seen at zero and negative gate voltages. This could be explained by electron migration in the gate region, which would not be present for other voltages. Importantly and rather disappointingly the bias stress effect, as seen in many NC solids, was observable in all devices, and could be measured independently of the memory gating effect. The bias stress effect is a shift in threshold voltage with application of a gate bias, which manifests itself as a stretched exponential decay in current, after a change in bias at the gate electrode [194]. The ramifications for this when measuring electrical response were summarised in the discussion of Figure 4.14. The physics behind the effect are still being investigated. The most thorough exploration to date in NC systems was by Luther et al. in 2008 [195]. This work established that the shift in threshold voltage was not caused by impurities or surface modifications of the oxide layer, concluding that the effect was a result of the change in charges being trapped on the layer of NCs closest to the oxide layer. Figure 4.19 illustrates the bias stress effect in these devices. In this measurement a constant $5 \mathrm{~V}$ bias was applied to the device and the current was measured, $V_{G}$ was stepped from $20 \mathrm{~V}$ then $15 \mathrm{~V}, 10 \mathrm{~V}, 5 \mathrm{~V}$, and $0 \mathrm{~V}$ twice, each gate 
voltage is applied for $\approx 55 \mathrm{~s}$. The current transients occur exactly at the $V_{G}$ shift. This effect is caused long term decays across subsequent memory

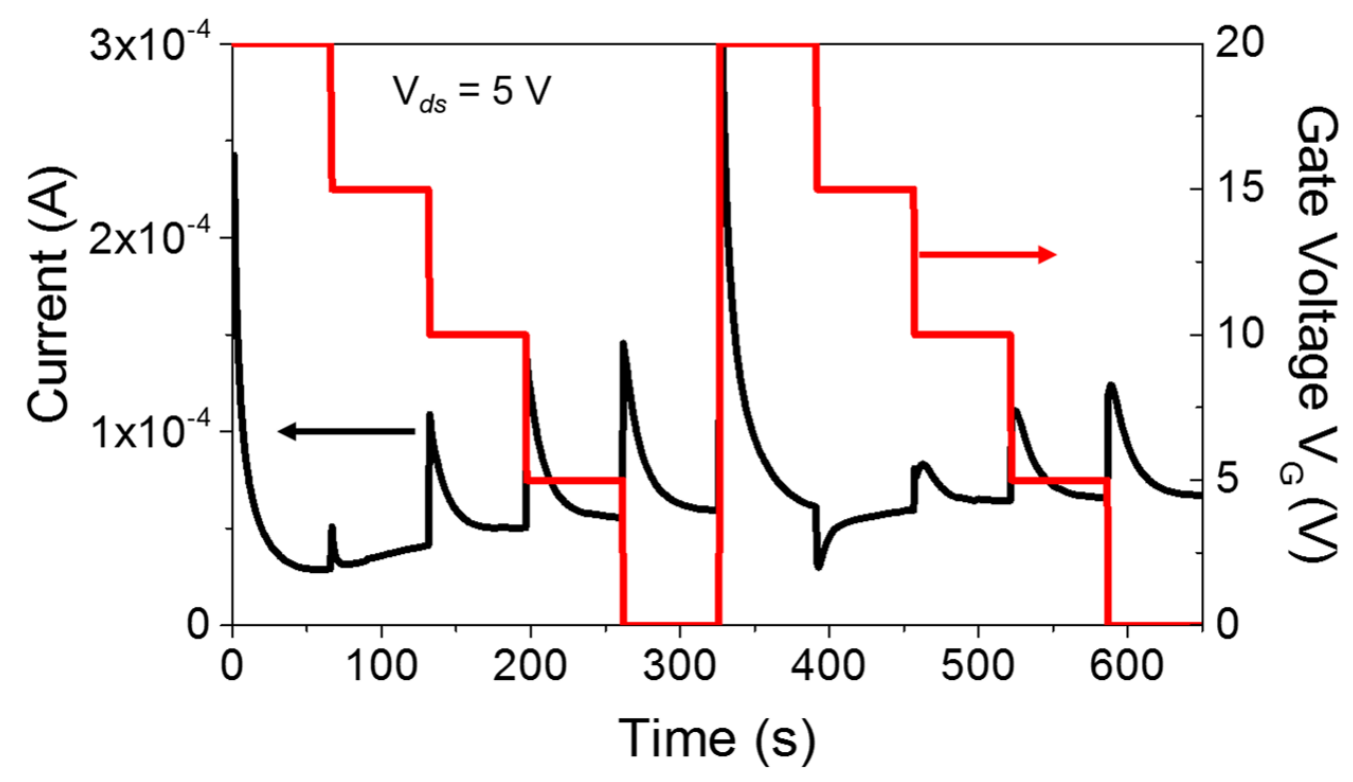

Figure 4.19: Current vs Time as $V_{G}$ is varied (right axis, red line). The bias stress effect discussed in the text causes sharp transients in current when it is changed, therefore limiting the use of the gate voltage as an additional tuning parameter.

cycles if the device were held at any gate voltage and thus substantially hinders the repeatability of the memory metrics over long run times. As such it degrades the behavioural stability of the device, although with the benefit of adding another control parameter.

\subsection{Discussion}

As has been shown these HgTe NC films oxidise over $60 \mathrm{~h}$ in air. As a consequence, long lived (trap lifetime $>$ relaxation time) hole trap states are formed which impart electrical memory to the material. Conduction in NC films is best understood through the phonon-assisted hopping model whereby nearest neighbor hopping $(\mathrm{NNH})$ dominates conduction at high 
temperatures, and variable range hopping (VRH) dominates below some critical temperature $[40,196-198]$. The probability of hopping occurring between any two possible states has previously been shown at Equation 1.22. The role of trap states in hopping is twofold. Mid-band traps offer lower barrier heights and thus aid hopping. They also serve to localise charge, providing recombination and capture centers [198,199]. As in bulk dielectric materials, long term trapped charges can cause hysteresis and other time dependent effects $[182,183,200]$. However due to the unique nature of hopping transport, the underlying mechanisms are rather different. The behaviour of these devices can be understood using a space charge limited current with a deep trapping model [201], which is consistent with trap mediated hopping transport. The underlying concept of this model is that the filling of deep traps within the material creates a screening field that acts to reduce the field at the injecting contact. Thus the behaviour of the trap population over time results in time dependent current density. The continuity Equation for such a system is [201]

$$
J(x, t)=\mu \rho_{f}(x, t) F(x, t)-D \frac{\partial \rho_{f}(x, t)}{\partial x},
$$

where $J(x, t)$ is the current density, $\mu$ is the effective carrier mobility in the system, $D$ is the diffusion co-efficient hlacting against current flow, in this case it is a constant fixed function of the mobility [201]. $F(x, t)$ is the electric field and $\rho_{f}$ is the free charge density. The free charge density being the sum total of charges that are not otherwise bound, a trapped charge is not part of the free charge density. This equation will be used to qualitatively test the measured response of the device under various temperature and light conditions to see if it also fits this previously published description of an NC film with long lived traps. 


\subsubsection{Hysteresis Dependence on Temperature and Illumi- nation}

The trend of conductivity with temperature is as expected for these HgTe NC films and is shown in Figure 4.20 [202-205]. For HgTe NC films there

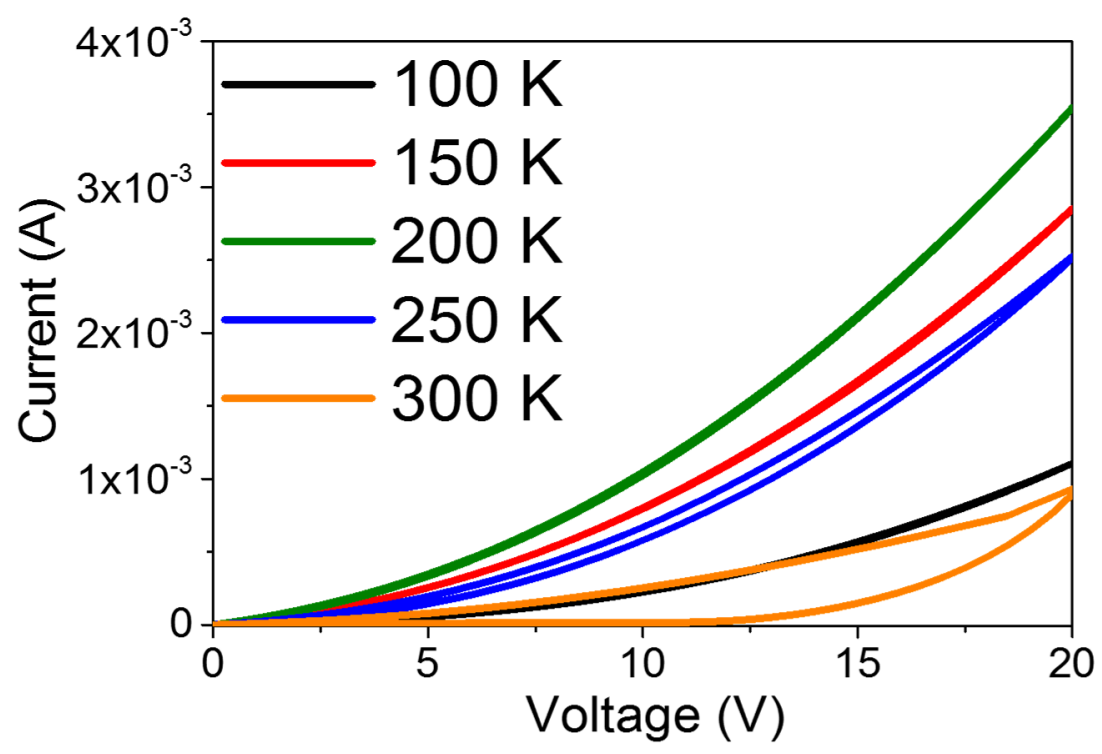

Figure 4.20: Graph showing the temperature dependence of the IV hysteresis for dark devices. Note that, the conductivity reaches a maximum at $200 \mathrm{~K}$, the hysteresis is only visible at $250 \mathrm{~K}$ and above, and that the $300 \mathrm{~K}$ conductance is the lowest.

is a peak of conductivity around $200-230 \mathrm{~K}$ and it then decreases with increasing temperature. For this discussion it can then be said that the mobility of the film at $100 \mathrm{~K}$ is greater than at $300 \mathrm{~K}$, since the measured current at $300 \mathrm{~K}$ will be aided by the increase of free thermal carriers. As shown by Figure 4.10 the hysteresis is larger in the positive quadrant and as such will be the focus of this discussion. 


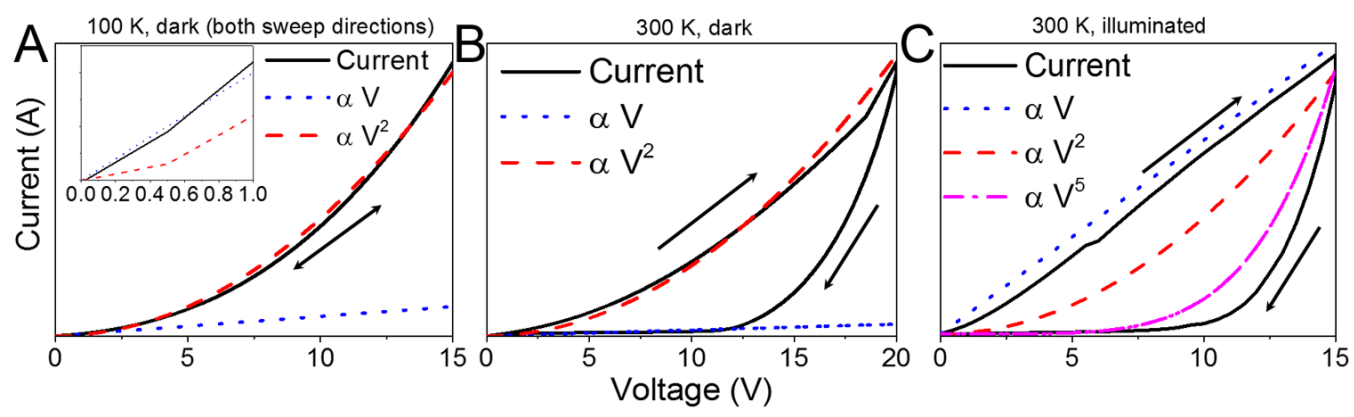

Figure 4.21: $A$, dark IV at $100 \mathrm{~K}$, no hysteresis, inset showing ohmic behaviour at low field. B, dark IV at $300 \mathrm{~K}$, hysteresis, forward sweep showing $I \propto V^{2}$, backward tending to diffusion. C, illuminated $(2.5 \mathrm{~mW})$ at $300 \mathrm{~K}$, hysteresis, forward sweep showing linear, high carrier density, backward tending to diffusion.

For $100 \mathrm{~K}$ in the dark, the IV curve (Figure $4.21 \mathrm{~A}$ ) displays no hysteresis and can be modelled as having an unchanging trap population that is not sufficient to prevent current flow through the device. The change in free charge density with time is

$$
\frac{\partial \rho_{f}(x, t)}{\partial t}=-\frac{\partial J(x, t)}{\partial x}-\frac{\partial \rho_{t}(x, t)}{\partial t}
$$

where $\rho_{t}$ is the trapped charge density. At these low temperatures the detrapping rate will be low and if the trap population is unchanging then the second term in Equation 4.7 disappears and the system is time independent. If $\rho_{f}$ is small throughout, due to a low number of thermal carriers $\left(n_{i}\right)$, then it will be dominated by injected carriers $\left(\rho_{f}(x)\right.$ varieswith $\left.F(x)\right)$ and the diffusion term can be neglected. We therefore would expect an $I \propto V^{2}$ relationship, as observed (Figure 4.21 A) [206]. As the temperature increases under identical optical and electrical conditions hysteresis is observed. At $300 \mathrm{~K}$ the hopping will be due to $\mathrm{NNH}$ and there will be $10^{6}$ more thermal carriers (for a bandgap of $0.324 \mathrm{eV}$ ). At the start of the IV measurement there will be some trap population that is higher than at 100 $\mathrm{K}$. The increased trap filling is due to an increased probability of a hole existing in the valence band, which can be filled, leaving a higher number 
of localised hole traps throughout the material. An increased trap density with temperature also explains the reduction in conductivity with increasing temperature. As the applied field increases, an $I \propto V^{2}$ relationship still exists (Figure $4.21 \mathrm{~B}$ ) because the space charge field is still increasing with voltage as before. However, when the applied field is decreased, a clear hysteresis is observed. At this point, in Figure $4.21 \mathrm{~B}$, the trap population in a region is now sufficient to create a screening field that reduces the effective applied field. In the NNH dominated regime, this screening field is sufficient to significantly reduce current since conduction is dependent now only on nearest neighbours, each filled trap has a larger screening effect. Along with changes in the direction of diffusion, as the space charge region retreats under decreasing voltage conditions, this screening effect gives rise to the hysteresis and the initial $I \propto V^{5}$ decrease once the applied voltage cycles from maxmium back toward zero. This quickly tends to diffusion based transport as shown by the shape of Figure 4.21 B. Over time, the screening region will thermally de-trap and the system will settle to the equilibrium trap filling for any arbitrary applied voltage (such as the read voltage). As such, the shape of the IV loop is dependent on the measurement time. Crucially this explains the behaviour shown in Figure 4.7. From the perspective of the IV curve this looks the same as the mechanism widely reported for a number of non-filamentary memristive systems [207-209], where the device is being operated in what has conventionally been referred to as the "RESET" process. Here, since the NC conduction is dominated by hole traps, the forward sweep gives the LRS mode and the backward sweep gives the HRS mode, wherein we observe an abrupt decrease in current.

Since the samples are thin compared to the absorption depth, generation and recombination will be present throughout the film under above band gap photoexcitation. Thus the dominant component of $\rho_{f}(x, t)$ would no longer come from the injected carriers and Equation 4.6 would thus pre- 
dict an ohmic ( $I \approx V)$ behaviour, that depends only on $F(x, t)$ as the other terms become constant. This is observed in Figure 4.21 C, where the hysteretic behaviour is still observed because the screening charge still remains when the applied field is decreased. If we describe this behaviour in terms of the memory cycle we would expect that when the read follows a positive erase voltage, then the system will show the HRS, as the injected charges see a screening field due to the trap filling that remains after the applied positive field is removed. Conversely, if the read voltage follows a negative write voltage, then the system will show an LRS as the injected charges see a region devoid of traps once the applied negative field is removed. Photoexcitation will cause both the HRS and LRS to erode over time as the trap filling tends to the equilibrium value for the applied read field eliminating the hysteresis effect. The model proposed above is summarised in Figure 4.22. 


\title{
A
}
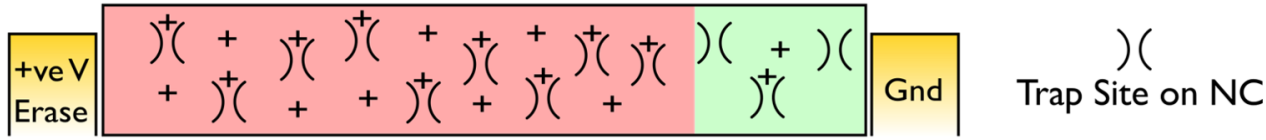

B
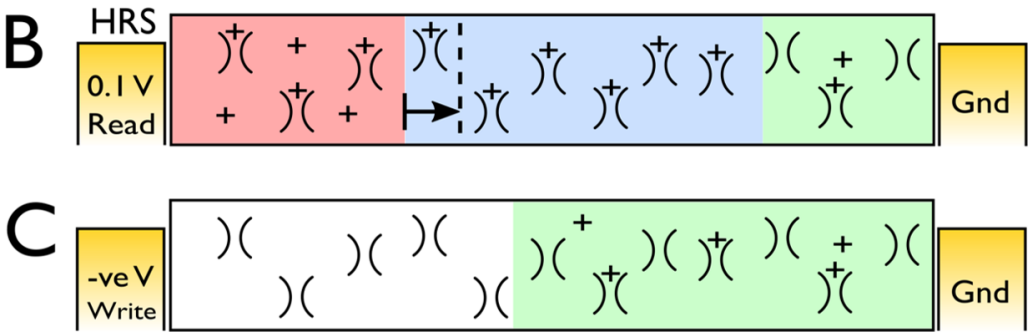

\author{
Diffusion \\ region
}

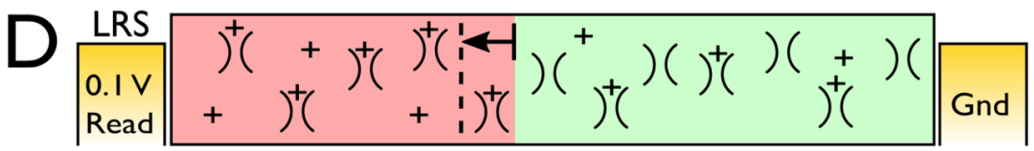

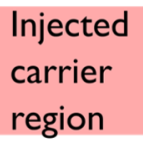

Screening field

Figure 4.22: $A$, during the erase step holes are injected and there is a high conduction region with a large amount of trap filling. $B$, when the read voltage is applied there remains a high degree of trap filling that screens the applied field. Thus the HRS is observed until the trap filling in this region settles to the equilibrium $0.1 \mathrm{~V}$ filling through recombination of photoexcited carriers and thermal de-trapping. The arrow indicates the steady-state trap filling that would be induced by the read voltage. Then $C$, the write step removes hole traps near to the electrode via injection of electrons, generating a region with low trap filling. D, The read voltage that follows senses the LRS as there are a large number of sites available to facilitate trap mediated hopping and a large number of vacant trap sites that can be filled by injected carriers. After some time the trap filling settles to the steady-state $0.1 \mathrm{~V}$ level, again represented by the dashed line. The difference between the initial conditions experienced by the read voltages define the HRS/LRS on/off variation. 


\subsubsection{Memory Dependence on Temperature and Illumina- tion}

If the behaviour of the hysteresis is dependent upon temperature and illumination then it is expected that when probing the device with the memory cycle, similar behaviour will be observed. The hysteretic dependence on temperature was shown in Figure 4.20. It is clear that there is no hysteresis below $250 \mathrm{~K}$ and above $250 \mathrm{~K}$ this effect then increases with temperature. There is an identical temperature dependence on the appearance of the memory effect, confirming that they are representative of the same mechanism, as is shown in Figure 4.23 B. This demonstrates that the memory effect becomes observable at $250 \mathrm{~K}$, however at $300 \mathrm{~K}$ in the dark there are insufficient carriers to overcome the low conduction (shown in Figure 4.20). For this reason there is no measurable on/off ratio at $300 \mathrm{~K}$ in the dark. This also explains why the memory appears to switch on when the device is illuminated (Figure $4.18 \mathrm{~A}$ ), as the illumination is required to provide sufficient charge carriers for it to be observed. To paraphrase it appears that the trap states are populated by temperature and measurable numbers of carriers at $300 \mathrm{~K}$ are provided by carrier generation.

The photoresponse in these highly oxidised films, occurring over seconds (shown in Figure 4.18 A), was noticeably slower than for newly deposited (i.e. non air exposed) films, that have a rise time of $50 \mathrm{~ms}$ (Chapter 3) [210]. Figure 4.23 A shows the dependence of the on/off ratio versus incident light intensity. Large HRS/LRS differences are only observed in devices that have sufficient light to generate free carriers but not so much light as to generate so many carriers that they instantly force the trap filling to the equilibrium level for the read voltage. This is what is occurring in Figure $4.18 \mathrm{~A}$. In the dark there is no memory effect yet in the light there are sufficient free carriers to give detectable conduction, however this same carrier density also causes the decay of the intial trap distribu- 

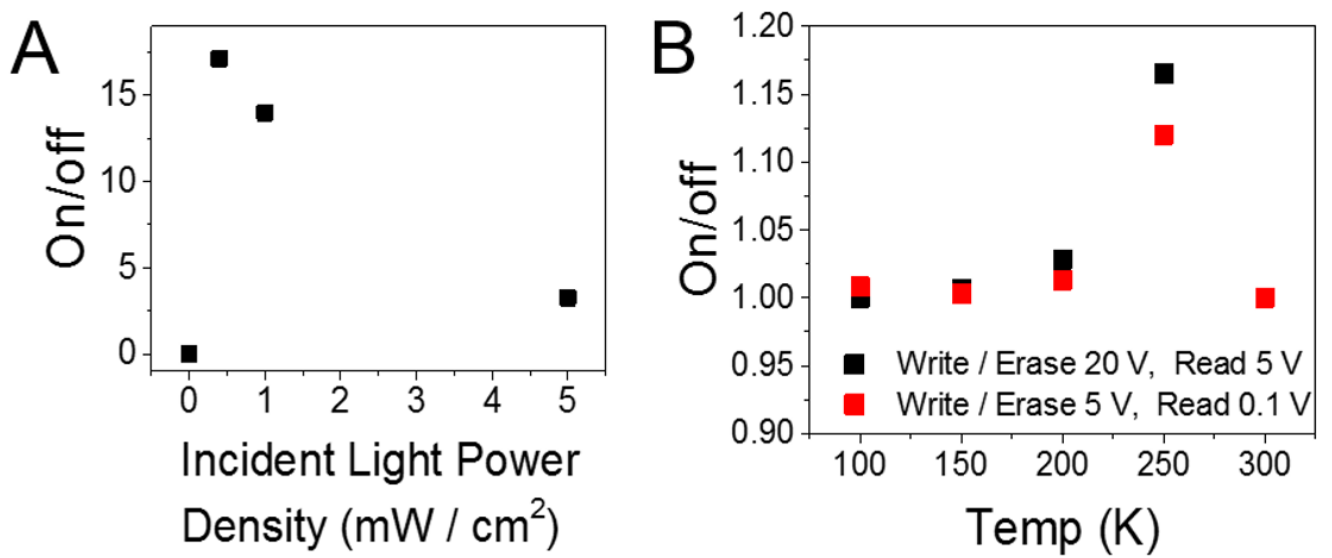

Figure 4.23: $A$, the change in on/off with incident white light power. The lack of $x$-axis data points is related to a lack of adjustability with the lamp controls. B, temperature dependence of on/off for dark devices, for two different memory protocols. This higher voltage protocol was used primarily to provide a higher current at $100 \mathrm{~K}$

tion and so causes the HRS and LRS states to decay. It is these decays of the HRS/LRS to the equilibrium conduction for $0.1 \mathrm{~V}$ that are clearly visible in the read steps. The performance of the "champion" device shown in Figure 4.15 was achieved by experimentally testing the best performing device under different light and voltage conditions. For the cycle shown the light level was $\approx 0.5 \mathrm{~mA} \mathrm{~cm}^{-2}$ resulting in a carrier density sufficient to give an easily identifiable on state but an off state that had very little current flow. The device was operated intermittently under these conditions for 4 days.

This model can be further experimentally verified as if the light is reduced during the write step there is a detrimental effect upon the subsequent LRS. This occurs as the lack of free charge carriers in the dark write step, makes it difficult to inject or withdraw holes from NCs further from the electrode. This reduces the degree to which the trapped charge population can be modulated, thus limiting the LRS/HRS current ratio, shown in Figure 4.24. If the LRS were only to do with the material and the volt- 
age there should be no change to the LRS current for identical light and voltage conditions, however there is a major difference in behaviour indicating that the LRS behaviour is heavily influenced by prior charge flow.

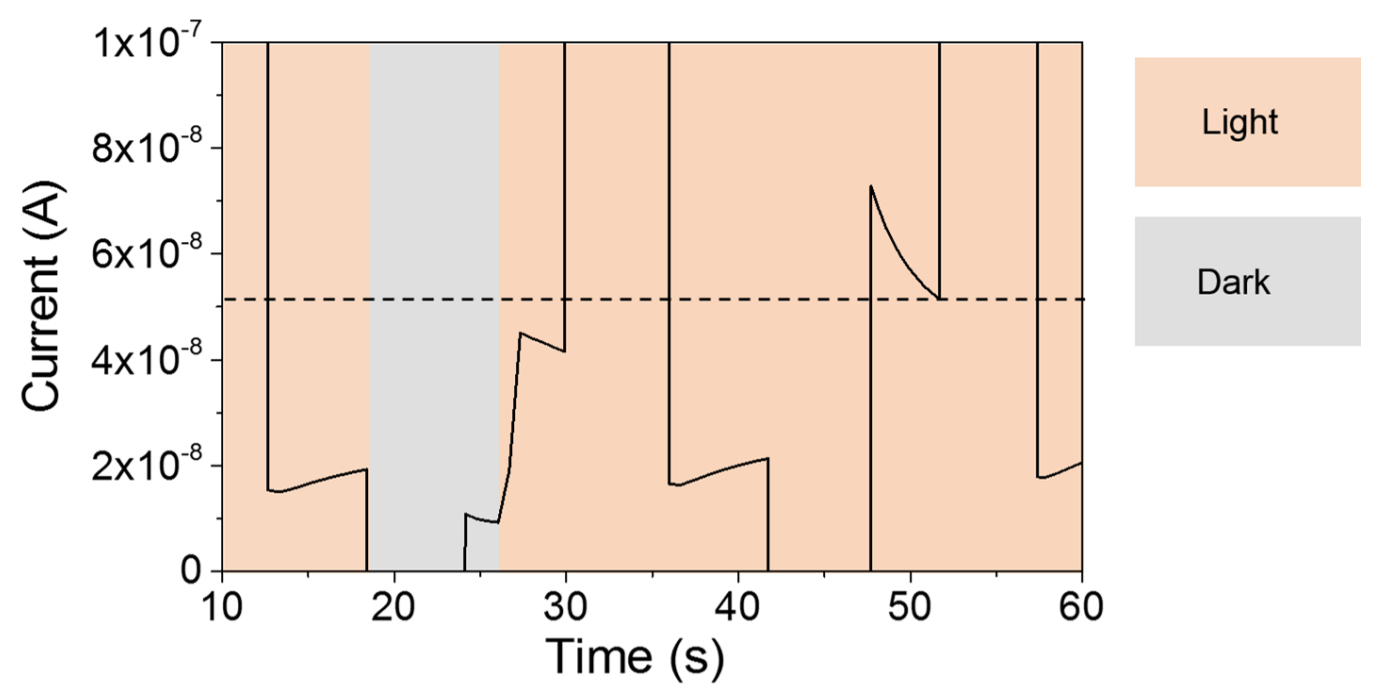

Figure 4.24: The change of LRS behaviour if written in the dark for the same device as shown in Figure 4.18. The dashed line is a guide for the eye to show that the dark written on state never reaches the current of the light written on state. As there is no illumination during the erase step the level of trap filling in the material is greater than that found after an illuminated erase pulse. There is still some region near the electrode that has no filled traps causing the intermediate LRS observed, however there will be a number of filled traps throughout the film that would normally have been erased by photoexcited carriers.

\subsection{Conclusion}

The model proposed and evidenced above, and summarised in Figure 4.22 fits with all observed data and the current understanding of the behaviour of oxidised NC films. Under intense white light $\left(>5 \mathrm{~mA} \mathrm{~cm}^{-2}\right)$ the conduction immediately (with respect to the probing measurement) reverts to the equilibrium read voltage state, as the population of filled or unfilled hole traps generated by the erase and write functions, respectively, 
are immediately returned to the equilibrium distribution. Thus the correct intensity of white light is needed to permit adequate generation of free carriers for good overall conduction, but not so many that the trap filling immediately returns to equilibrium following the write/erase pulses. In these devices this condition was found to be optimised at $\approx 0.5 \mathrm{~mA} \mathrm{~cm}^{-2}$. Adequate surface traps are also needed for preparation of a memristive device. If a less volatile and more tightly binding surface ligand is used, such as $\mathrm{As}_{2} \mathrm{~S}_{3}$, the hysteresis is eliminated (Chapter 3). The stark differences in electrical behaviour that result from differing surface treatments indicate that the observed effects are a result of surface trap states, that have been shown to be oxygen based. The charge carriers exist in the quantumconfined electronic levels of the individual NCs and the conduction mechanism is due to hopping, however due to the long lived hole trap states the bulk device behaviour is understood with a bulk regional approximation model. The trap density in the material is dependent on the number of excited carriers but the trap lifetimes are long compared to the hopping time. As the excitation rate defines both the free carrier density and the trap recombination rate in the film it must be tuned to balance these two processes in order to give good operation. We can now see that our device can be described by Equations 4.3 and 4.4 and is therefore a memristive system. The measured current at any time is described by the filled trap density, the free carrier density and the applied voltage. Importantly the change of the state variables ( $\rho_{t}$ and $\rho_{f}$ ) over time is dependent upon the variable itself, the applied voltage as well as the excitation intensity $\left(J_{P h}\right)$. Inserting into Equations 4.3 and 4.4 we suggest that under constant conditions the parameters that describe these devices fit into the memristance state equations as

$$
\begin{gathered}
I=G\left(\rho_{t}, \rho_{f}\right) \cdot V \\
\frac{d \rho_{t}}{d t}=g\left(\rho_{t}, V, J_{P h}\right), \frac{d \rho_{f}}{d t}=\frac{\partial J(x, t)}{\partial x}-\frac{d \rho_{t}}{d t} .
\end{gathered}
$$


These equations only state that from experimental evidence it appears that the measured current at any time is defined by some function of the trap filling, the number of free carriers and the voltage. The exact nature of $G$ would require further research, likely starting with a simulation of this system. Using these equations as a basic model it can be seen that a higher excitation rate will cause a faster decay towards the equilibrium trap density. With zero excitation in the dark there are insufficient carriers to detect any HRS /LRS difference at the read voltage. With white light excitation there are more free carriers, a higher filled trap density and a measurable on/off effect. Increasing the amount of carrier generation above some peak value causes increased detrapping and the measured current tends to the equilibrium filled trap density too quickly to give a meaningful memory response. Thus, although the memory state can be set by emptying or filling the traps, this state cannot be measured without some incident white light to increase the carrier density and it is this increased free carrier density that in turn eliminates the LRS or HRS states through trap filling and recombination, respectively. While this effect limits the longevity of the memory states, the unique light and gate sensitive hysteretic behaviour of these materials may suggest other materials and mechanisms that could be employed to create true passive memristors using colloidal NCs, with better metrics. In short the dependences shown in the equations above are required to explain the range of observed behaviours, and further research is definitely needed to provide further robust detail in this area.

Here we have demonstrated pure NC devices that are fabricated and operated in air with robust on/off ratios over long time periods and repeated read steps. The devices are activated by light and the on/off ratios can be tuned by changing the light conditions. Response to applied gate voltages is clear and provides a pathway to gate controlled NC memory devices. Mechanisms that cause the observed effects are suggested and imply that 
this is a true memristive system with readily identifiable state variables, further understanding of which will lead to enhanced understanding of both NC trap mechanics and memristive behaviour. Whether or not this memory behaviour is useful notwithstanding, it is unequivocal that these films have trap states that are sufficiently long lived to affect the macro electrical state of the system. With these oxidised films this trapping has been intentionally maximised, however working under the assumption that no ligand environment provides complete passivation, all HgTe QD devices will have a number of hole trap states in the film. Indeed this is evident in the transient photoresponse data in Chapter 3 for $\mathrm{As}_{2} \mathrm{~S}_{3}$ capped films as the trap states cause slow recombination. This opens up the possibility the density of trap states can be optimised so that sufficient trapping of one carrier type allows photogain but without severely undermining transient response, as there would be an inherent trade off between those two mechanisms. 


\section{Chapter 5}

\section{Multi Pixel Imaging with Photogain Enhanced Detection}

Parts of this work have been submitted for review in an article titled:

Photogain enabled Thermal Imaging in Multi Pixel Planar Quantum Dot Devices

\subsection{Motivation}

Following from the work in Chapter 3 the next obvious step was to attempt to shrink the pixel size, pattern multiple pixels and develop a means to electrically read out from multiple pixels at the same time. Combining these three features would allow a device to act as a proof of concept for an actual multi pixel array that can be patterned in an ambient environment with highly simple and robust, and therefore cheap, techniques. The intent was to show three key results that would lay the groundwork for further development of arrays of planar, PC pixels. This being the opposite to the currently more popular approach within the published literature that involves QDs within a vertical, stacked structure and operation in the PV mode. The three aims were, firstly, to produce a 2-D array of sub mm pix- 
els. Secondly, to show that the variation between pixels when operated electronically was small enough that it warranted further research into miniaturisation, and that the physical inhomogeneity between pixels created from solution patterning was not a barrier to functionality. Thirdly, to demonstrate that an array of pixels could "image" an object, and demonstrate spatial resolvability. An example of a state of the art, commercial microbolometer that is the ultimate target of this work can be found at Reference [211].

\subsection{Background}

The current state of the art in QD devices uses the PV approach to demonstrate metrics on par with current commercial solutions, but uses QDs as the sensitizer layer in place of epitaxial bulk semiconductors [54,212]. This method has the key advantages of integrating with current camera technology and offers an easily scalable pixel density. More importantly this integration of QDs into a photodiode structure has recently shown excellent results. The Guyot Sionnest Group at the University of Chicago has recently demonstrated $1 \mathrm{kHz}$ optical response, $10^{11}$ Jones detectivity and actual images of body heat using an optical rastering system in conjunction with a single, cryogenically cooled, $200 \mu \mathrm{m}$ pitch pixel [213,214]. These are performance metrics that rival current state of the art commercial systems based on cooled bulk semiconductors. This approach however, does not take full advantage of the single step deposition and patterning that CQDs allow to further drive down device cost and fabrication bottlenecks. Thus the second approach is to optimise and downsize simple PC QD detectors for use in the MIR [107]. In a stroke this solves many of the problems of cost and fabrication complexity, due to the single step solution deposition that colloids allow. Much work has been done with this approach, the performance breakthrough being achieved by Lhuillier et al. with the introduction of inorganic ligands [99]. Building on this it has 
subsequently been shown that the spectral selectivity through size tuning of the QDs can be carried through into the final device and that the devices can achieve $1 \times 10^{9}$ Jones detectivity and $0.9 \mathrm{~A} \mathrm{~W}^{-1}$ responsivity at room temperature $[111,215]$ (Chapter 3$)$. Crucially, photogain, which can deliver $>100 \%$ EQE (Equation 1.23) and thus enable relatively noisy devices to detect small photon flux, was first reported in PC HgTe devices by Böberl et al. in 2007 [108]. This was followed up by another report of gain by Chen et al. in 2014 [216]. Both works showed an increasing gain with decreasing incident power, although both of these devices were operating in the NIR below $1600 \mathrm{~nm}$ rather than the MIR beyond $3 \mu \mathrm{m}$. There are very few reports on the use of colloidal QDs in multi pixel devices. The best to date is the work by Ciani et al. [21] who coated a commercial digital read out array with HgTe QDs and showed that when cooled to $95 \mathrm{~K}$ it could image room temperature objects. This is another good example of the first, PV, approach to QD MIR devices. In terms of the second approach it has been shown that $200 \mu \mathrm{m}$ QD pixels can be patterned onto 3 pixel electrode structures however these pixels were not electrically contacted for measurement [111]. Finally, Cryer et al. demonstrated that spray coating could deliver multiple large pixels onto a single device, and that, when measured individually these pixels maintained their expected bandgap dependent opto-electrical response [215].

\subsection{Experimental}

The HgTe QDs used in the devices reported in this Chapter were fabricated with the same methods as those used in Chapter 3. The only change was the addition of $5 \mathrm{ml}$ of $3.0 \mathrm{M} \mathrm{HCl}$ to the quenched growth solution, as has been shown to be beneficial for CdSe [217]. This was done with an aim to better passivating the surface by bonding a $\mathrm{Cl}^{-}$ion to any unpassivated surface sites that remain after the DDT has attached to the surface. As much as DDT is the primary ligand it is possible that a number of $\mathrm{Hg}^{+}$ 
surface sites remain unpassivated due to steric hindrance, the smaller $\mathrm{Cl}^{-}$ ion could possibly reach any sites that remained unpassivated. Figure 5.1 shows the difference in colloid stability after 3 months of storage in air between a $\mathrm{Cl}^{-}$treated and an un-treated suspension, that are otherwise identical.
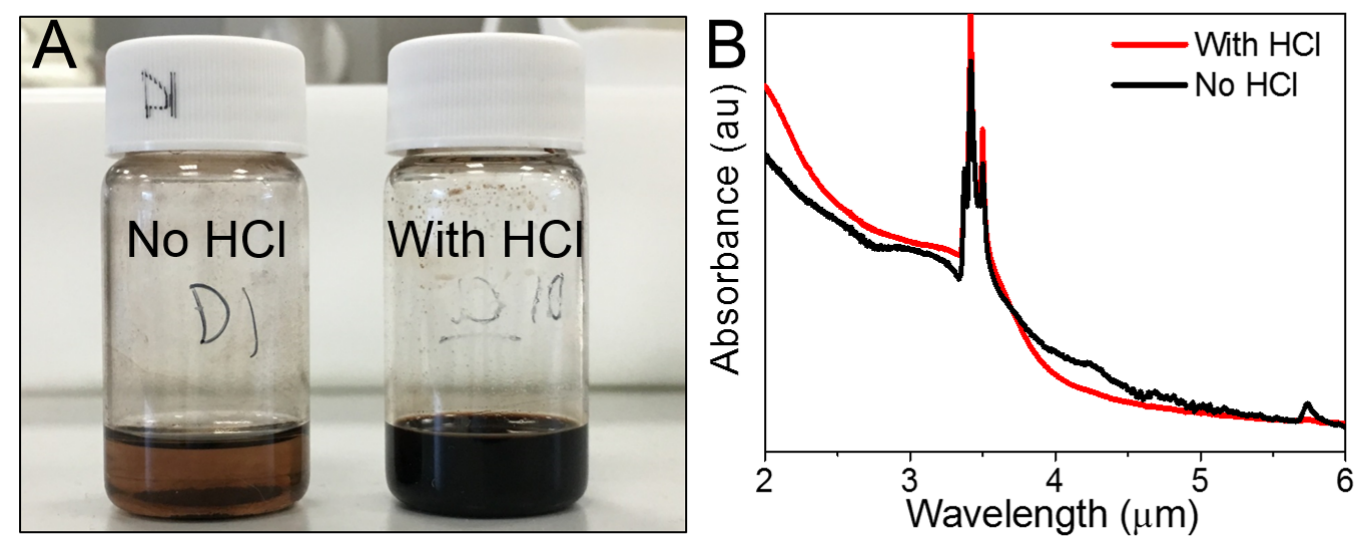

Figure 5.1: $A$, the same $Q D$ s, separated after quenching where the sample on the right is treated with $\mathrm{HCl}$. Both samples are then crashed and re-dispersed twice under identical conditions. After 3 months of storage in air the $\mathrm{HCl}$ treated sample is still fully suspended in chloroform, whereas the un treated sample has mostly precipitated out. B, ATR absorbance spectra of the colloids in $\mathrm{A}$. The $\mathrm{Cl}^{-}$passivated sample has a visibly reduced Urbach tail, indicating that there are fewer trap states or surface defect sites that "smear" the bandgap.

\subsubsection{Device Design}

The key design constraint to consider when designing the multi pixel chip was that a planar IDE device structure has two terminals. This means that both terminals on each IDE needed to be routed to an external connection. Therefore, it was decided that the multi-pixel device would have 16 external connections. This gave a manageable density of contact pads around the outside of the chip that could work with the available equipment, but still allowed an array of pixels to be fabricated that all had two connections. In order to maximise the number of pixels each pixel was connected 
to a uniform rail, which meant 15 remaining connections and therefore 15 active pixels. As the samples were best suspended in chloroform the mask itself had to be fabricated from a material that was impervious to chloroform, metal being the most obvious solution. The smallest available drill bit that could work with aluminium was $0.7 \mathrm{~mm}$ in diameter therefore that was the chosen pixel size. The work shown here would be easily upscalable with an inkjet printing capability which could deliver pixels on a 10 $\mu \mathrm{m}$ scale. To maximise the responsivity of the pixels the IDE spacing was chosen to be $5 \mu \mathrm{m}$ as this was the smallest reproducible feature size on the photolithography setup available.

\subsubsection{Silicon Devices}

PET substrates, as used in Chapter 3 due to the macro surface roughness were not suitable for either sub $10 \mu \mathrm{m}$ features or accuracy over an entire $15 \mathrm{~mm}$ square device. As such the first devices fabricated with the new design were made on functionalised silicon using the same process as found in the previous two Chapters. An unpatterned example of a silicon substrate device is shown in Figure 5.2. Silicon oxide on silicon wafers are a well proven technology so it was relatively easy to get the feature quality shown in the figure. Figure 5.2 E gives an idea of the quality of the IDEs. The use of silicon based devices however was ultimately futile as the delicate silicon oxide dielectric, $300 \mathrm{~nm}$ thick on these devices, was not sufficiently robust to cope with the spray coating process. Numerous attempts were made to remedy this by ensuring there was no physical contact between the spray mask and the substrate but none were successful. Figure 5.3 shows some examples of the finished, and damaged silicon devices after patterning. 


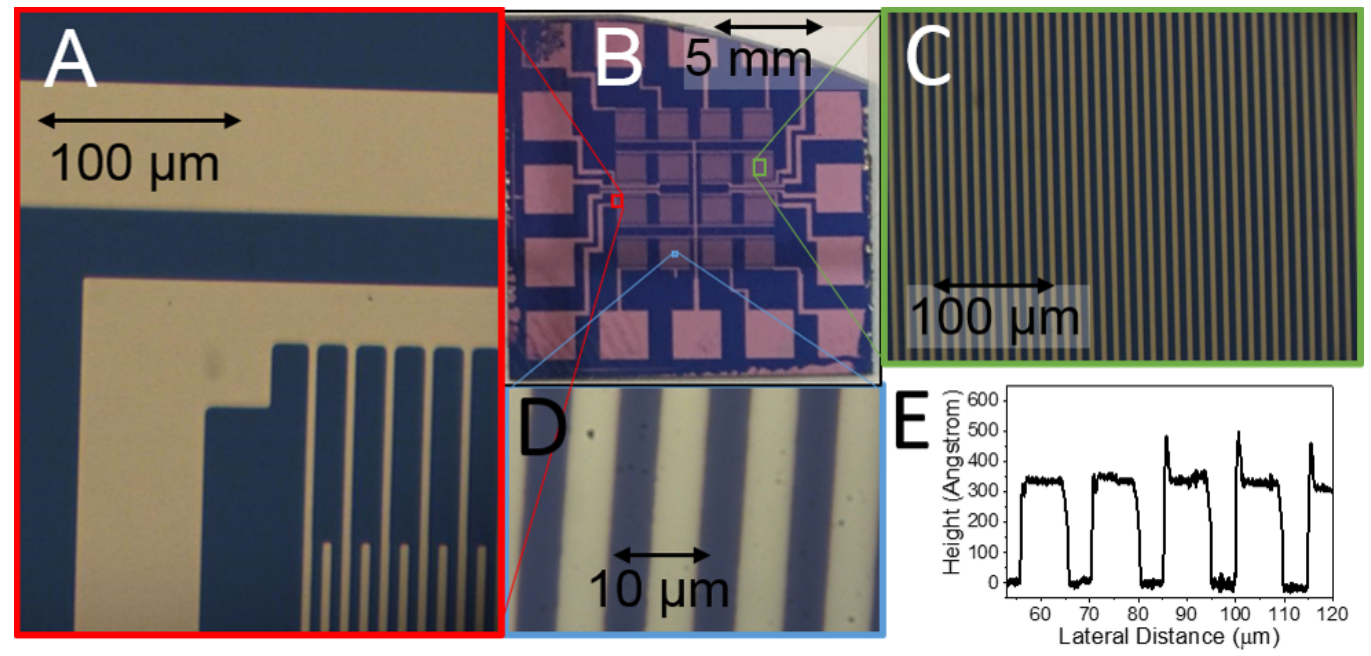

Figure 5.2: Typical multi-pixel device fabricated on silicon. A, the area in red from $B . B$, an un-patterned device on silicon. $C$, the area in green from $B . D$, the area in blue from $B$. E, a profilometer measurement of an area similar to $D$, measured perpendicular to the IDEs.

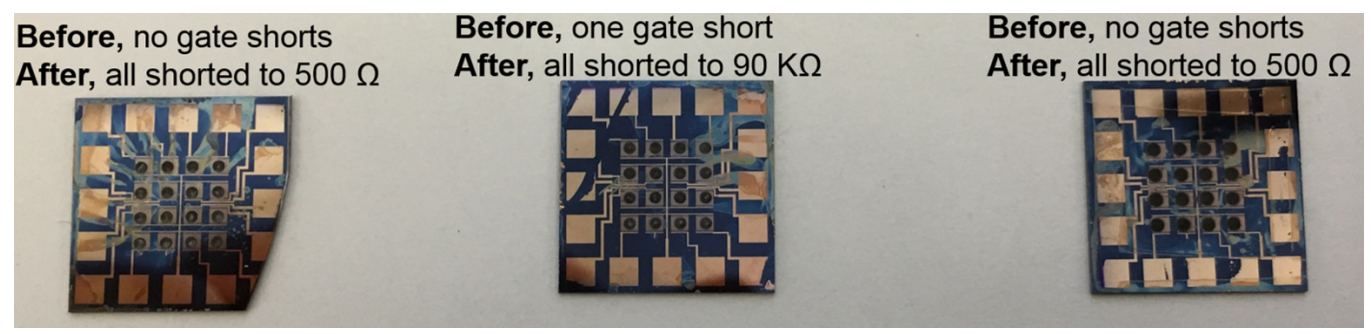

Figure 5.3: Three successfully patterned silicon devices where each is annotated to show the number of measured gate shorts before and after patterning.

\subsubsection{PMMA Devices}

The PET devices developed in Chapter 3 demonstrated that hydrophobic polymers worked well as substrates for thiol capped HgTe QDs. The downside with PET was that the specific sheeting used had a large surface roughness and very quickly absorbed EtOH. As silicon wafers did not work with the spray coating method it was required to find another substrate material. An ideal material would offer the surface properties of PET with the surface homogeneity and solvent resistance of a silicon 
wafer. Four potential substrate materials were investigated, polypropylene, acrylic (polymethylmethacrylate (PMMA)), "smooth" PET and polycarbonate. Of these the PMMA showed the best response to the photolithographic process developed for the PET, delivering repeated $5 \mu \mathrm{m}$ features on the second device fabrication run. The rest of this Chapter therefore uses devices that have $5 \mathrm{~mm}$ thick PMMA sheeting as the substrate.

The complete experimental fabrication process for the devices reported here is as follows. HgTe QDs were synthesised using the common method. $72 \mathrm{mg}$ of $\mathrm{HgCl}_{2}$ and $6 \mathrm{~g}$ of octadecylamine was degassed at $120{ }^{\circ} \mathrm{C}$ under vacuum for $1 \mathrm{~h}$. The temperature was reduced to $60^{\circ} \mathrm{C}$ under nitrogen and $0.2 \mathrm{ml}$ of TOP:Te in $10 \mathrm{ml}$ of $80 \%$ oleylamine was injected. The temperature was increased to between 90 and $110{ }^{\circ} \mathrm{C}$ (hotter gives larger particles) and held at that temperature for $5 \mathrm{~min}$. The reaction was quenched by the addition of $31 \mathrm{ml}$ of tetrachloroethylene and $4 \mathrm{ml}$ of dodecanethiol. This was stirred for $10 \mathrm{~min}$ and then $5 \mathrm{ml}$ of $3.0 \mathrm{M} \mathrm{HCl}$ was added and stirred for $5 \mathrm{~min}$. The QDs were crashed with methanol, cleaned twice and then dispersed in $6 \mathrm{ml} \mathrm{1:1} \mathrm{hexane:chloroform}$.

The PMMA substrates were $15 \mathrm{~mm}$ by $15 \mathrm{~mm}$ of $5 \mathrm{~mm}$ thick acrylic sheeting. Prior to photolithography the substrates were cleaned in ethanol and then coated in positive resist (AZ1518 MicroChemicals, $4000 \mathrm{rpm}$ for $60 \mathrm{~s}$ followed by $90{ }^{\circ} \mathrm{C}$ bake for $10 \mathrm{~min}$ ). Following UV exposure, developer (AZ326 MicroChemicals 3:1 in DI water) was used to develop the substrate. $\mathrm{Cr}(5 \mathrm{~nm})$ and $\mathrm{Au}(45 \mathrm{~nm})$ were deposited by evaporation. Lift off was done via sonication in ethanol for $30 \mathrm{~min}$.

The substrates were placed in a stencil mount with $0.8 \mathrm{~mm}$ diameter holes centred over each pixel. Spray deposition was achieved with a nitrogen fed spray gun at a stand-off distance of $12 \mathrm{~cm}$. Following deposi- 
tion the entire mount was submerged in $10 \mathrm{mg} \mathrm{As} \mathrm{S}_{3}$ in $20 \mathrm{ml}$ 1:1 propylamine:ethanol for $30 \mathrm{~s}$ then rinsed in ethanol. These devices were fabricated with 3 layers.

Electro-optical measurements were completed with a bespoke 16 pin clamshell mount. The universal contact was connected to the SMU-Hi output of an NI PXIe 4138 SMU. Each pixel was then connected in series with a $\mathrm{K} \mathrm{k} \Omega$ resistor. The voltage dropped over each resistor was measured by an NI PXIe 4303 multi-channel DAQ with individual voltage probes on each line. Each pixel/resistor line then terminated at the SMU-Lo output. Measurement line noise was $10 \mathrm{nA}$ with no device connected. Unless otherwise stated the optical path consisted of the Omega-BB4A calibrated black body (BB) source cavity opening, $10 \mathrm{~cm}$ of air, a plano-convex $\mathrm{CaF}_{2}$ lens and the device. The set up was calibrated for incident power and solid angle using a Thorlabs S401C thermal power detector, spectrally accurate beyond the upper transmission limit of the $\mathrm{CaF}_{2}$ lens. Due to the complexity and bulk of the 16 channel measurement mount the device had no other shielding from external stimuli, i.e. it was open to changes in background thermal and light level. Although this sounds far from ideal it allows an honest report of device performance in real-world conditions. PC devices are particularly robust in this regard as the fluctuations in background flux just add to the constant bias derived, non-negligible dark current [18]. To be classified as a detectable signal in this work the criteria used here was that any change must be greater than $3 \sigma$ in current, where that $\sigma$ in current has been experimentally determined in a measurement period following the test. As such all results shown below are above the total noise of the system. To give an example of the measurement methodology, Figure 5.4 A gives an example of the total noise in a channel, including all background noise, for a period following an optical measurement. The standard deviation of this signal is $1.33 \mu \mathrm{A}$. Therefore in accordance with the methodology used throughout, only current changes that are $\geq 4 \mu \mathrm{A}$ 
and are time correlated with an optical signal will be used as measurement points. As can be seen from Figure $5.4 \mathrm{~B}$ the current change in $0.5 \mathrm{~s}$ with a BB target at $150{ }^{\circ} \mathrm{C}$ is just greater than $4 \mu \mathrm{A}$, (to be specific the threshold that is applied is therefore $3 \sigma$ at $2 \mathrm{~Hz}$ ). Therefore that temperature BB is detectable. Lowering the target temperature may still result in a time correlated current increase however if it is smaller than $4 \mu \mathrm{A}$ it is not classed as a signal. Incident optical signals were created by a calibrated blackbody
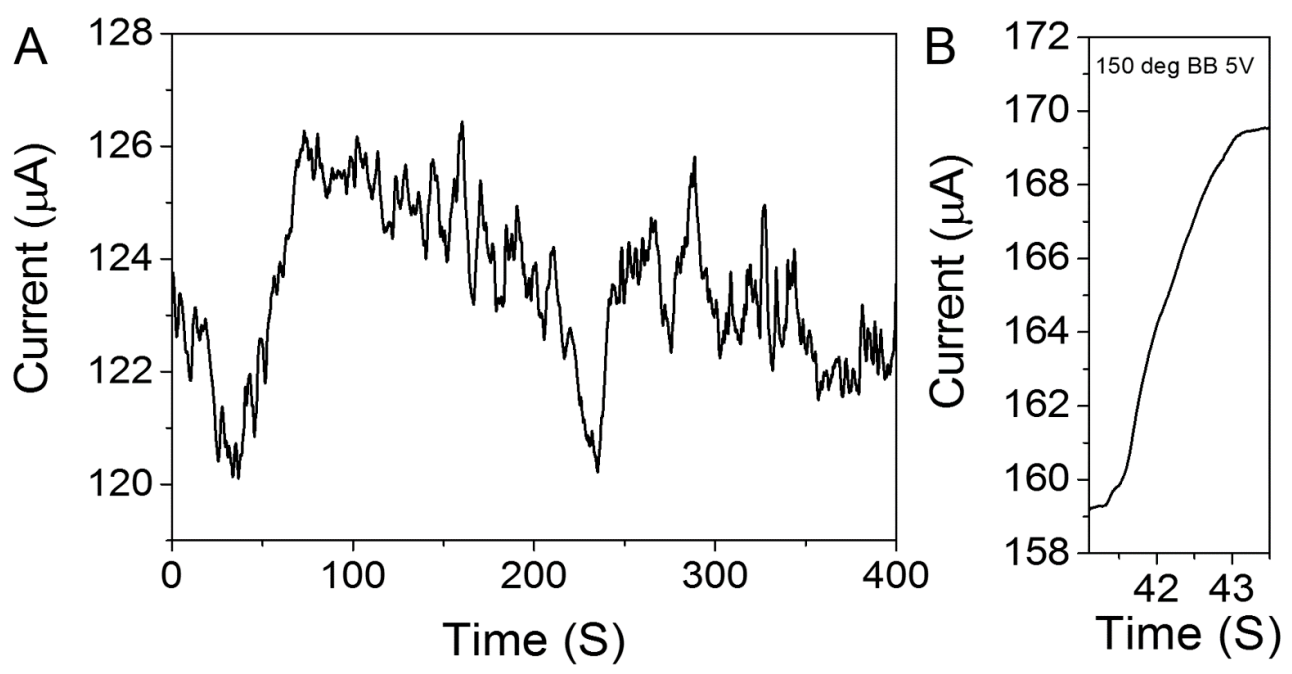

Figure 5.4: $A$, the total noise in channel 6 for a period following the optical tests. There are no changes to the image field during this time, the sample rate is $100 \mathrm{~Hz} . B$, the total current change for a $150^{\circ} \mathrm{C} B B$ being exposed.

(BB) test source with a low temperature limit of $373 \mathrm{~K}$. A single $\mathrm{CaF}_{2}$ lens was used to modestly increase the flux onto the detector, however in terms of total collected flux transferred to an image plane at the device, this setup is in no way comparable to a fully functional lens system as used by Ackerman et al. [213,214]. Unless otherwise noted the device is being run at $+5 \mathrm{~V}$ bias.

The voltage data was recorded at $100 \mathrm{~Hz}$. The time varying data presented in this Chapter has been passed through a $20 \mathrm{~Hz}$ low pass filter to 
remove power line noise and a 3 point median filter. Using a low pass filter with such a low cut off frequency will cause a noticeable rounding of on signals but was necessary to cope with the noisy measurement environment. To display multi pixel information the imshow package for python was used, which allows numerical values data to be represented in a 2-D grid with a scale bar. As such it perfectly allows the response of multiple pixels to be shown intuitively.

\subsection{Results}

The absorbance spectra of the QDs used in the devices are shown in Figure 5.5. The four QD samples here spanned the MIR and the measured bandgap of the film after ligand exchange is shown in the figure key, the nomenclature of the samples used here is carried on throughout this Chapter. 


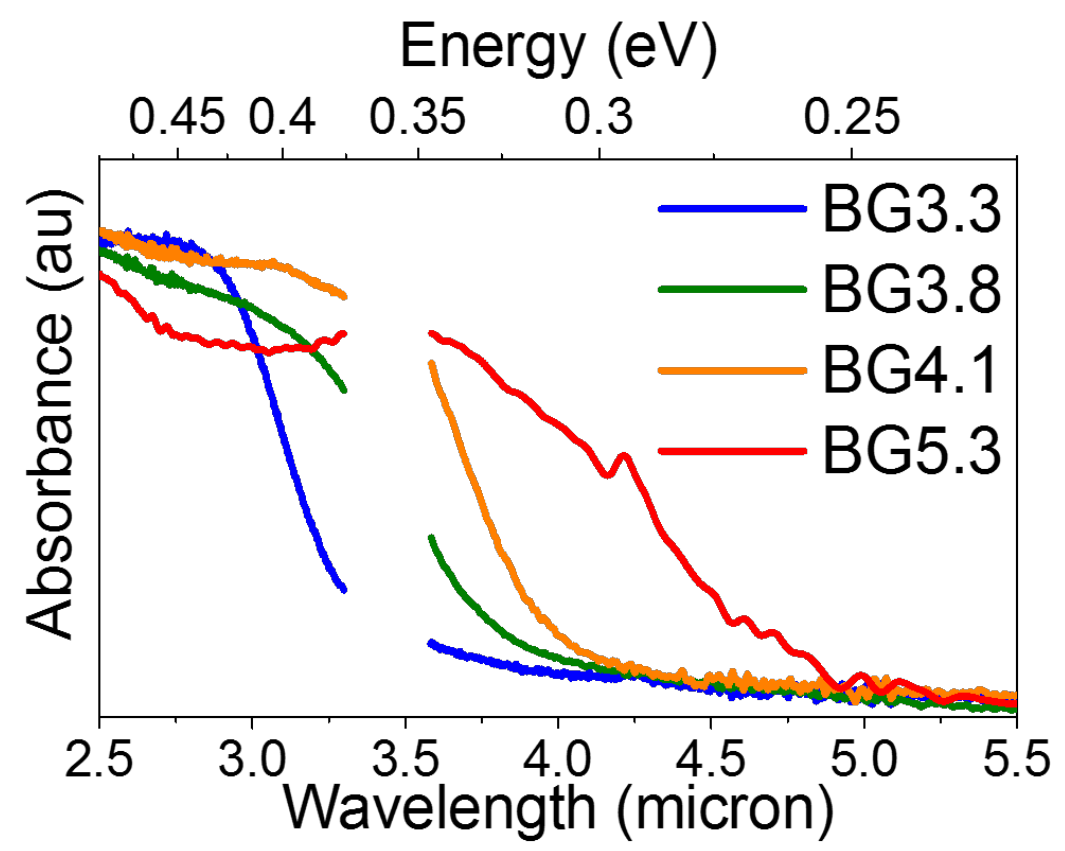

Figure 5.5: ATR spectra of the QDs used in the devices reported in this Chapter, the $C-H$ stretch has been blanked out. The labels refer to the band gap in $\mu \mathrm{m}$ of the QD films after ligand exchange, not the band gap of the ATR measurements., the labels are used throughout this Chapter. 
The QDs are deposited on the device by spray coating through a stencil, then ligand exchanged whilst in the stencil mount. A typical device after patterning is shown variously in Figure 5.6. SEM images Following
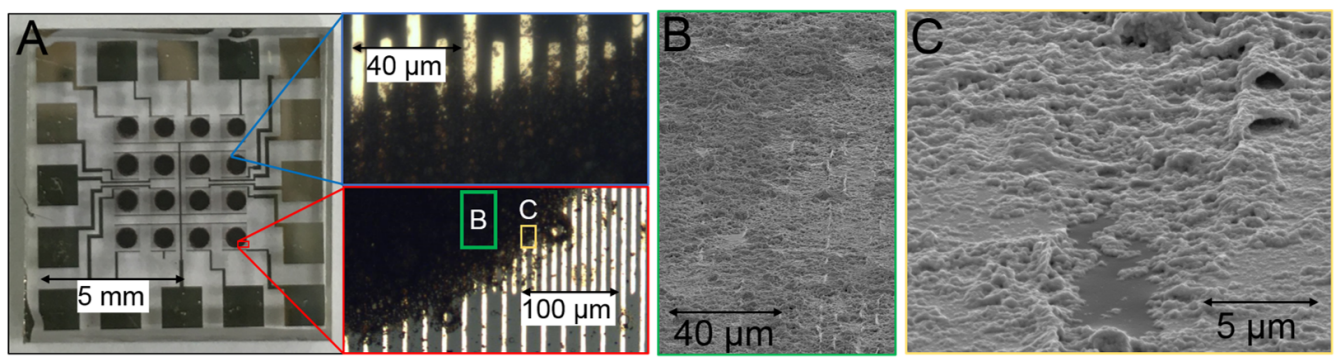

Figure 5.6: $A$, optical images of a typical PMMA device after patterning. Shown at three different magnifications. $B$, SEM image of area $B$ from $A$. C, SEM image of area $C$ from A

ligand exchange the band edge is preserved in the film with a redshift between 100 and $300 \mathrm{~nm}$, therefore each QD remains physically separated, but closer to its neighbours following ligand exchange, as shown for BG5.3 in Figure 5.7. The bandgaps used throughout for calculating incident flux are the larger measured film band gaps. 


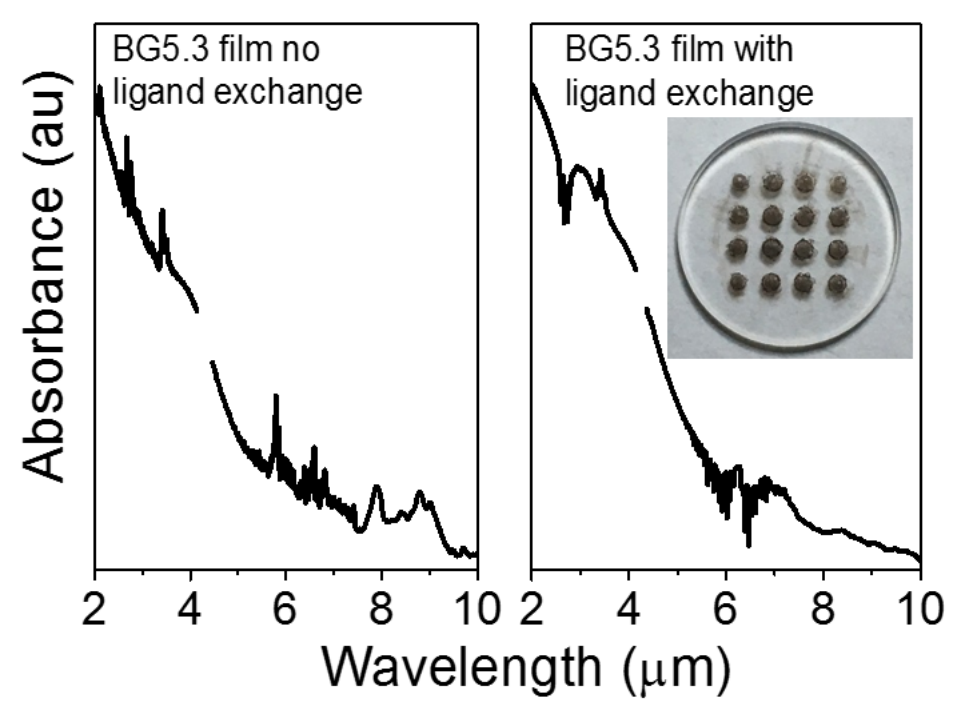

Figure 5.7: FTIR path absorbance measurements of a spray coated film of BG5.3 on $\mathrm{CaF}_{2}$, identical to that found on the device. The left hand plot shows a spray coated film without ligand exchange, the right hand shows a film prepared under identical conditions but treated with $\mathrm{As}_{2} \mathrm{~S}_{3}$. The inset shows an image of the film on the $\mathrm{CaF}_{2}$ substrate, as measured.

\subsubsection{Multicolour Imaging}

In the same way that the addition of red, green and blue colour information provided another "dimension" of information to visible technologies, it should be facile to use multiple sizes of QD to provide a similar development in thermal imaging systems. If the detector is suitably calibrated, then as well as giving intensity information i.e. an electrical signal proportional to all absorbed photons above the bandgap, it will be possible to give both intensity and temperature information. This is demonstrated in Figure 2, reconstructed from devices with the 4 different band gap materials shown in Figure 5.5. Figure 5.8 shows the 4-colour pixel response to 4 temperatures of target from $500{ }^{\circ} \mathrm{C}$ to $200{ }^{\circ} \mathrm{C}$. All the pixels can see the target and have a current response that is shown as a proportion of the current response, in that pixel, to a $700{ }^{\circ} \mathrm{C}$ target. All colours show a 
variation in response between pixels of the same colour which exists due to the inhomogeneity in films of solution deposited nanomaterial solids and is to be expected, best illustrated by BG4.1 in Figure 5.8.

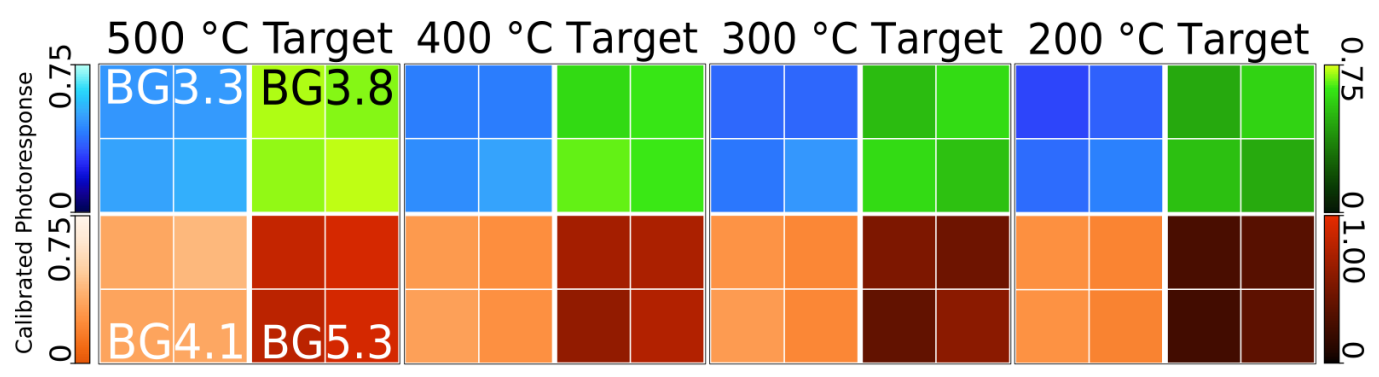

Figure 5.8: Visual representation of four colour response to four different target temperatures. The pixel response has been calibrated by normalising as a fraction of the current response of the pixel to a $700{ }^{\circ} \mathrm{C}$ target. Scale bars and colours are constant throughout, note that the BG5.3 scale bar is 0-1, whereas the other colours are $0-0.75$, due to the lower absolute but higher calibrated photoresponse across the range with low bandgap NCs. The subtle variation between pixels of the same colour is a real effect from the device. In this target temperature range, all of the pixels can detect radiation from the target.

The concept of multi spectral detection in the MIR is complicated by the spectral blackbody distribution of any hot object and the spectral performance of the detector. However, in these materials each colour had a threshold temperature below which no response could be detected above noise. Below this threshold temperature there were insufficient above bandgap photons for any signal to be detected. For BG3.3 this threshold temperature was $150{ }^{\circ} \mathrm{C}$. Figure 5.9 A shows the same pixels as in Figure 5.8 responding to a $140{ }^{\circ} \mathrm{C}$ BB. BG3.3 cannot see the target but the other pixels are still providing intensity information. The multispectral nature of the device has given information about the temperature of the target. For a single small bandgap material this would not be the case. The only difference would be a reduction in intensity and as such it could also be caused by the object moving further away or being covered. However, with the multi spectral system shown here it is clear that the second object is cooler than the first. Regardless of emissivity the image cannot be hotter than $150{ }^{\circ} \mathrm{C}$, as BG3.3 can never see below $150{ }^{\circ} \mathrm{C}$. These results are, 
clearly, for an idealised situation with a BB source. Real world objects have in general a reduced emissivity and spectral detection must also account for the absorbance of the intermediate medium, however this could be easily achieved via proper calibration and a well understood optical system. The threshold temperature for BG3.8 was $120{ }^{\circ} \mathrm{C}$ and thus when exposed to a $110^{\circ} \mathrm{C}$ target both BG3.3 and BG3.8 no longer give a detectable signal. Figure 5.9 A also shows the expected reduction in signal current for the remaining colours (same scale as Figure 5.8), again pixel to pixel variation is clearly evident. One obvious use of this technology would be the addition of a "body temperature pixel" to a thermal camera, a spectral overlay that at no optical conditions can see a BB at $38^{\circ} \mathrm{C}$, i.e any target that can be seen by that pixel cannot be a human body.

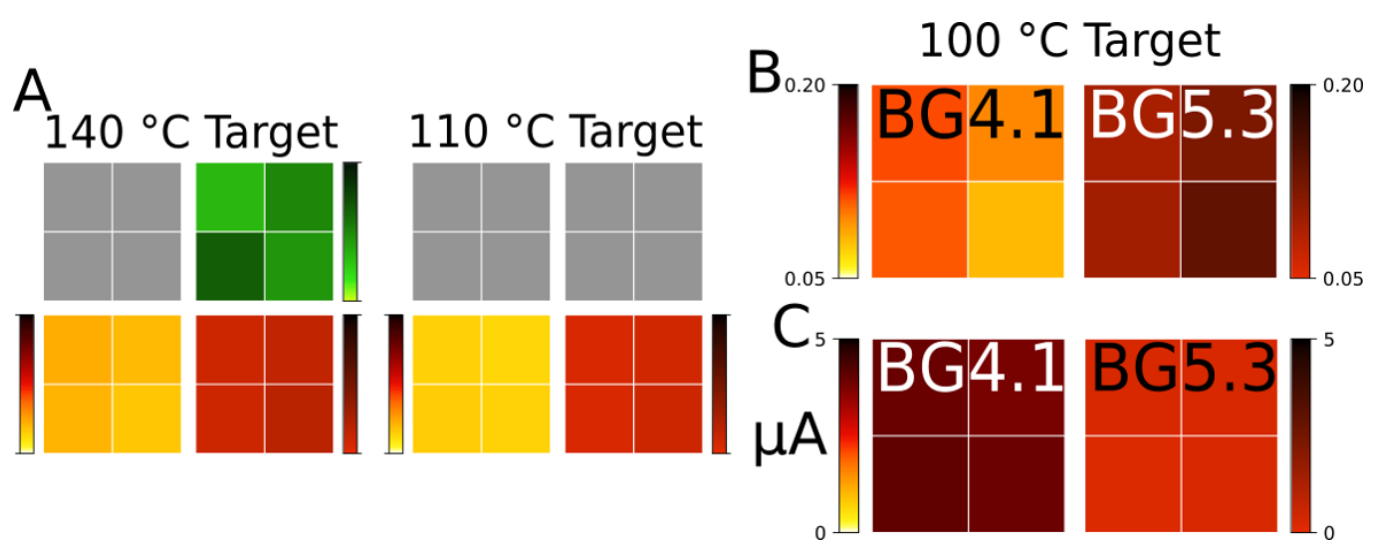

Figure 5.9: Low temperature target detection with four colours, the bandgap notation carries from Figure 5.5, grey represents no detectable signal, device uncooled, at $25^{\circ} \mathrm{C}$. $A$, These plots follow the same scheme as Figure 5.8. BG3.3 cannot see either target, and BG3.8 cannot see the $110^{\circ} \mathrm{C}$ target. This occurs as the devices no longer have a current response that is greater than $3 \sigma$ of background. Can be considered as an extension of Figure 5.8 to lower temperatures. $B$, this shows the normalised response of 4 pixels of $B G 4.1$ and BG5.3 to a $100{ }^{\circ} \mathrm{C}$ target, using the relative calibration method it appears as though BG5.3 is far more sensitive than BG4.1. C, this shows the absolute response of 4 pixels of BG4.1 and BG5.3 to a $100{ }^{\circ} \mathrm{C}$ target, even though BG5.3 has a larger bandgap than BG4.1, the absolute current response is an orders of magnitude higher for the BG4.1 pixels. 
In Figure $5.9 \mathrm{~B}$ the target temperature is reduced further to $100{ }^{\circ} \mathrm{C}$, a point where the temperature difference between the device and the target is only $75^{\circ} \mathrm{C}$. This sensor to target temperature difference is considerably less than the difference between human body temperature and an electrically cooled detector $\left(\approx 200{ }^{\circ} \mathrm{C}\right)$, achieved in spite of the exponential increase in thermal carriers with device temperature that add noise. Figure 5.9 B shows the normalised current response with a higher fidelity scale bar, and in terms of the calibrated response the larger bandgap BG5.3 has the highest sensitivity at these low target temperatures. Figure $5.9 \mathrm{C}$ shows the same data but with the absolute current values. BG4.1 has absolute current response in $\mu \mathrm{A}$, compared to the 100s of nA of BG5.3. As such, counterintuitively, the smaller bandgap material offers superior low target temperature detection than the larger bandgap material. The higher conductivity of the BG4.1 film means that even though the total incident flux is lower than for BG5.3, the current response is stronger.

\subsection{Discussion}

For PC detectors operating under constant bias there is always some current flow. To be detectable an optical signal must generate some change in current that is larger than the change in current due to any background optical signal and the noise of the device, which from this point will be called the total noise. The performance limit of a PC detector is then easily understood as being the point at which there are not sufficient incident photons to cause a current change larger than the noise. For BG3.3 one $\sigma$ of total noise was $\approx 1 \mu \mathrm{A}$ (see $\mathrm{SI}$ ), which at an EQE of 1 is equivalent to a total incident flux of:

$$
\text { photons } s^{-1}=\frac{1}{E Q E} \times \frac{I_{p h}}{e}=1 \times \frac{1 \times 10^{-6}}{1.6 \times 10^{-19}}=6.25 \times 10^{12} \text { photons }^{-1}
$$


Which, using the self-imposed criteria of $\Delta \mathrm{I} \geq 3 \sigma$, means that at an EQE of 1 the total incident flux at the pixel must be $\geq 1.875 \times 10^{13}$ photons s $^{-1}$ in order to see a signal. For a device with a bandgap of $3350 \mathrm{~nm}$ (BG3.3 film), a pixel area of $5 \times 10^{-7} \mathrm{~m}^{2}$, a solid angle of $0.0371 \mathrm{sr}$ and a BB source with an emissivity $(\epsilon)$ of 0.99 one can use the Planck equation for photon radiance $(\mathrm{SI})$ to find that the minimum observable temperature for a $\mathrm{BB}$ changing from completely obscured to completely unobscured should be $277^{\circ} \mathrm{C}$. It can be seen from Figure 5.10 A that our device with BG3.3 is able to see $\mathrm{BB}$ temperatures down to $150{ }^{\circ} \mathrm{C}$. Therefore, our device must be operating with an EQE greater than unity. In PC systems this is entirely allowable as the applied bias creates the electric field that moves the carriers and an EQE greater than unity means only that an electron/hole cycles through the circuit multiple times before its corresponding hole/electron partner reaches the opposite electrode. There are three main physical requirements that must be satisfied for photogain through charge trapping to occur. They are, that both contacts are ohmic, that both carriers are free in the material and that the trap lifetime of one of the carriers is much greater than the other $[107,218-220]$. In our previous work on charge dependent memory in HgTe devices we demonstrated that all these conditions are indeed met with this material (Chapter 4). Figure 5.10 A shows the $\mathrm{EQE}$ range, for $\mathrm{BG}$ 3.3. The inset shows the spectral incident flux on the device for $150{ }^{\circ} \mathrm{C}$ and $700{ }^{\circ} \mathrm{C}$ target temperatures. It should be noted that not only does the $700{ }^{\circ} \mathrm{C}$ flux include photon energies down to $\approx 1.24$ $\mathrm{eV}$, compared with $0.62 \mathrm{eV}$ for the $150{ }^{\circ} \mathrm{C}$ target, it also has 3 orders of magnitude more photons. Gain in the film must then be increased as total photon flux decreases or as the maximum energy of individual photons decreases. The inset also shows the raw current-time plots for the exposure to the shown flux, where the time response of the current decreases as the gain increases. Further study will be required to see if this is an intractable feature of high gain devices. To summarise, the EQE increases above $100 \%$ at a target temperature of $\approx 350{ }^{\circ} \mathrm{C}$ and reaches a maximum 
value of $1940 \pm 290 \%$ when exposed to a $150{ }^{\circ} \mathrm{C}$ target. Below this target temperature the current response drops below $3 \sigma$ of noise.
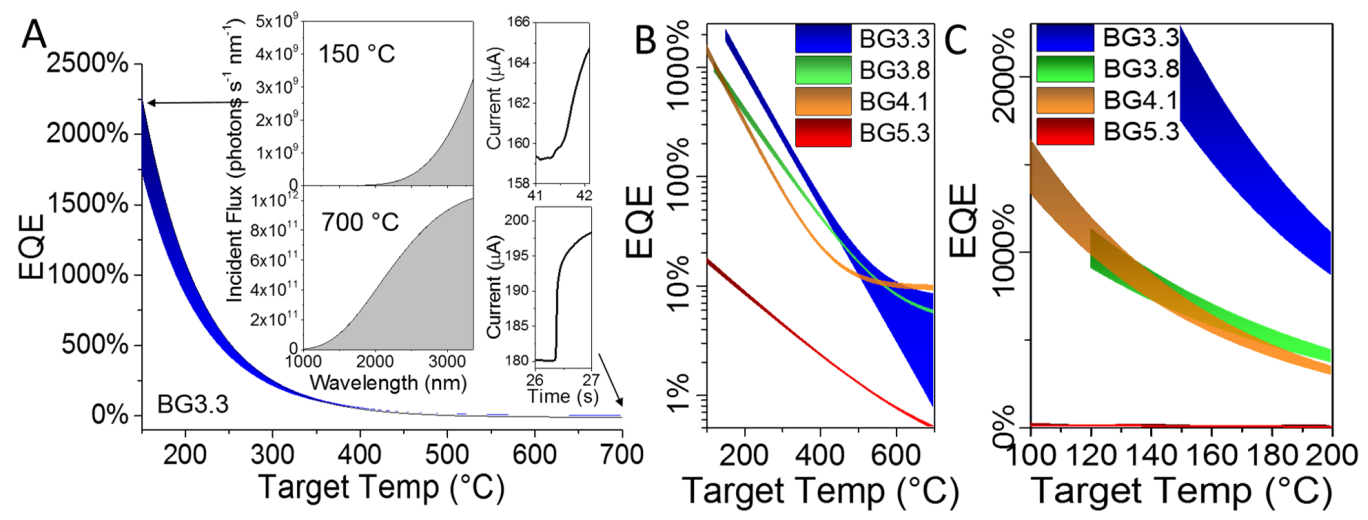

Figure 5.10: $A$, the EQE curve for BG3.3, the shaded area represents the error in the calculated $E Q E$ value. Inset shows the spectral incident flux and raw current change caused by exposure that combine to give the EQE value, for $700^{\circ} \mathrm{C}$ and $150^{\circ} \mathrm{C}$ targets. $B$, semi$\log E Q E$ curve showing the EQE of all 4 materials for the full target temperature range. $C, E Q E$ plot for target temp between $100^{\circ} \mathrm{C}$ and $200^{\circ} \mathrm{C}$. Note the cut off temperatures for $B G 3.3$ and BG3.8. In all figures the shaded area is the error region.

Figure $5.10 \mathrm{~B}$ shows the full range of EQE for all 4 colours used in this work. This range is calculated using the uncertainty in the bandgap estimation to give a minimum and maximum flux and the uncertainty in the solid angle given by the uncertainty in the optical calibration of the system. It is immediately obvious that BG5.3 never demonstrated an EQE > $100 \%$, but did still show an increase of EQE with reducing incident flux. It is instructive to look at the performance of these devices in the high temperature limit $\left(700{ }^{\circ} \mathrm{C}\right.$ target) as that is the regime where the majority of results for these materials have been published to date. For BG3.3BG5.3 the performances at $5 \mathrm{~V}$ bias yield responsivities of $0.22,0.13,0.23$ and $0.015 \mathrm{~A} \mathrm{~W}^{-1}$ respectively. These values are better, but within the same range, than previously published values for similar air exposed materials. The best performance is shown by BG4.1, displaying very good metrics for $\mathrm{a}>4 \mu \mathrm{m}$ bandgap material. Figure $5.10 \mathrm{C}$ shows the EQE range for 
low target temperatures, in this region all but BG5.3 are showing large EQE and thus must be operating with photogain. There is not a definite relationship between QD size and EQE across the colours, most likely due to differences in film quality, ligand coverage and oxygen exposure at the QD surface. The general trend is that smaller NCs display a greater maximum EQE. This can be explained with a simple geometric argument as if the trap density per dot and trapping rate is constant, films of smaller dots will require more hops between electrodes. Therefore, a film of smaller NCs will trap each individual carrier for a longer total time compared to a film of larger dots, thus increasing the observed gain. However, there are a few competing effects. Given the drastic reduction in gain between the three smaller dots and the largest, it is likely that the trap states causing gain have, as the bandgap reduces, become sufficiently close in energy to the valence band that they are now more easily depopulated by de-trapping.

The behaviour shown in Figure 5.10 also unequivocally demonstrates in all the colours that there is a relationship between the effective trap lifetime of holes and either the total photon flux or the energy distribution of the carriers, or indeed both. This is not unprecedented as it has been shown that the effective trap lifetime in these materials is inversely related to incident light intensity [125], due to larger fluxes reducing the trap time of holes, through interaction with a photogenerated carrier. This observation also agrees with the numerous reports of an increase in responsivity as the total incident power decreases $[108,215,216]$. The EQE dependence on the energy of the highest energy individual photons was investigated by the use of a $2400 \mathrm{~nm}$ long pass (LP) filter that blocked all photons with an energy $>517 \mathrm{meV}$. Figure $5.11 \mathrm{~B}$ shows the current response for two separate devices to the same source temperature range with and without the LP filter in place. The $y$-axis has been plotted as total incident photon flux. Both of these plots show the same trends in current response 
with and without the longpass filter in place. If high energy photons were more likely to reduce trap time then the current change with the filter in place would be higher and this is not observed. This strongly implies that gain is more strongly affected by the number of photons than by the energy of those photons, thus "hot carrier" specific effects are not dominant in this material.
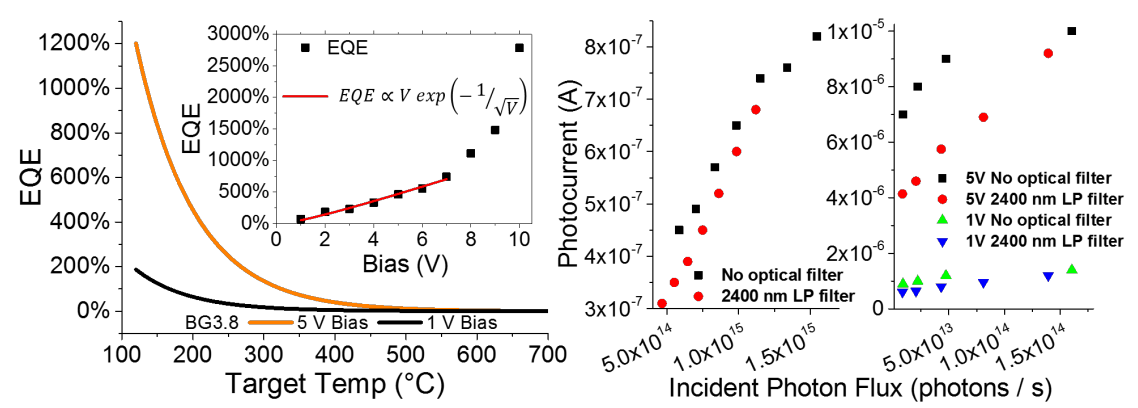

Figure 5.11: $A, E Q E$ of $B G 3.8$ device under $5 \mathrm{~V}$ and $1 \mathrm{~V}$ bias, the inset shows the behaviour of a film under constant optical flux but operated at various bias voltages, the fit line shows field driven hopping behaviour. B, the current change with incident flux plots for two devices, showing the behaviour for incident optical spectra with and without the highest energy photons (>517 meV).

In Figure 5.11 A we look at the bias dependence on EQE for BG3.8 under two bias conditions. As would be expected for a PC device the EQE is always lower for a lower bias voltage. The inset to Figure 5.11 A shows how the EQE changes with bias at a fixed photon flux. As with all films of nanomaterials, the limiting factor on conductivity is carrier movement between individual QDs not carrier movement, if it exists, within each QD. In these films, at $>1 \mathrm{~V}$ bias and at room temperature, the conduction mechanism is dominated by field driven nearest neighbour hopping (NNH). The QDs are well within the strong confinement regime $\left(r_{Q D}^{H g T e}<r_{B o h r}^{H g T e}\right)$ therefore the degeneracy within each QD is determined by the 0-D density of states and carrier movement within each QD is not a factor. This hopping mode 
will be $G \propto \exp \frac{-1}{\sqrt{E}}$ with applied field [40], therefore $I \propto V \exp \frac{-1}{\sqrt{V}}$, where for an unchanging photon flux, as is the case here, $I \propto E Q E$. The relationship in Figure 5.11 A inset fits this behaviour below $7 \mathrm{~V}$ and then deviates, moving to a stronger dependence on applied field. Using EQE as a proxy for conductance is valid as long as the hole trap lifetimes are bias independent. It therefore appears that at fields above $1.4 \times 10^{6} \mathrm{~V} \mathrm{~m}^{-1}$, in this sample, there is another driver for increased photogain beyond field driven carrier cycling, possibly an increase of trap lifetime.

Pixel to pixel variation in the electrical behaviour of QD films is one obstacle to reliable multi pixel devices. Indeed, when developing these devices the use of a single spin coated film over the entire device gave a pixel to pixel variation in measured resistance that covered 3 orders of magnitude. The spray coating method used for the final devices reported here was optimised by varying the solution concentration, spray stand off and stencil mount configuration in order to reduce pixel to pixel variation. However, as can be seen in Figure 5.8 and Figure 5.9 there is still noticeable variation between pixels on the same device. After process optimisation the variation in measured pixel resistance of the best performing devices was reduced to $\pm 50 \%$. One hurdle to prototyping these devices was the requirement to show a device where every pixel on the device functioned correctly. This is shown in Figure 5.12 A, showing the normalised current response to a $700{ }^{\circ} \mathrm{C}$ BB source being exposed, for every pixel. This may seem facile but it is a vital fabrication stepping stone to overcome if this concept is to be further miniaturised.

It can be argued that the real functionality of any imaging system is dominated more by the optics than the sensor, and the optics used here, are in an imaging sense, non-existent. It is still important however to show that the spatial separation of pixels at the device can deliver some spatial resolution in the signal response. Figure $5.12 \mathrm{~B}$ shows both the raw current data and how this maps spatially to the pixels for a device that only sees 


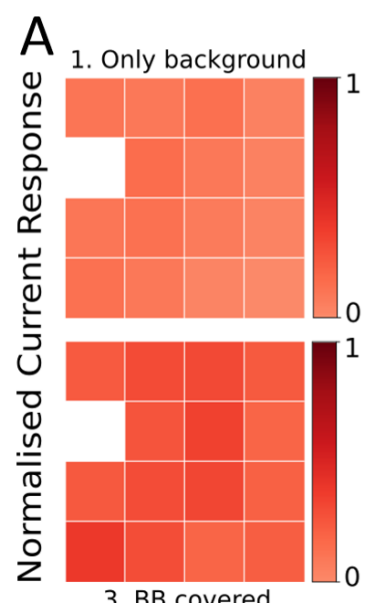

3. BB covered

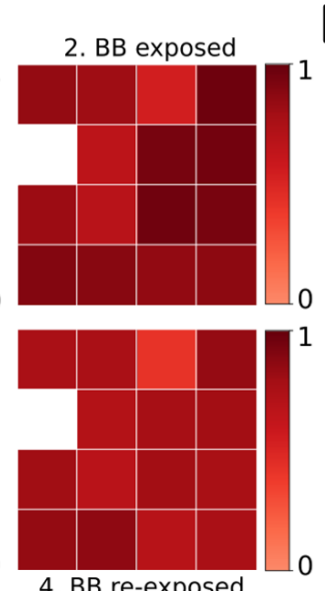

4. BB re-exposed
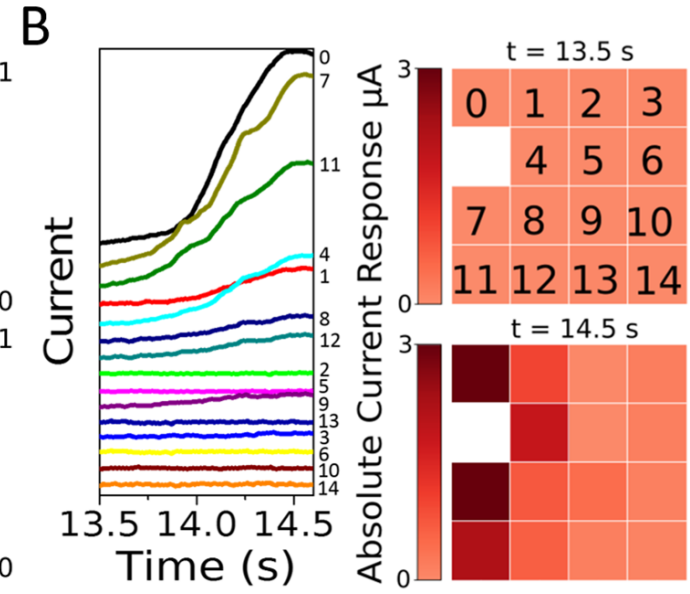

Figure 5.12: $A$, normalised current response of a device with 15 (out of 15) working pixels to two exposures of $a 700^{\circ} \mathrm{C} B B . B$, current-time curve and mapped pixel response to an exposure of a vertically occluded $B B$ at $700{ }^{\circ} \mathrm{C}$. The channels are annotated $0-14$, the channels have been vertically separated in the graph for clarity.

half (vertically) of the BB source compared to that seen by the detector in Figure 5.12 A. There is a clear distinction between the pixels on the side of the device that is exposed compared to the other side. This may seem trivial but it should be noted that a BB is an extended divergent source and the collimation in the system is limited at best.

\subsection{Conclusion}

Using only basic photolithography, readily available polymer substrates and a spraycoated layer of QDs this work has demonstrated device response that can only exist due to high levels of photogain in the film, delivering upon some of the oft-mooted reasons that QD devices are the future. The sensitivity to objects that have a temperature within $75{ }^{\circ} \mathrm{C}$ of the device temperature for an uncooled device is unprecedented and is possible due to the unique electrical behaviour of QD films. Furthermore, it has been shown that solution patterning, not just solution deposition, can 
deliver imaging potential. Further development in these materials must now concentrate on maintaining the performance, increasing device speed through ligand tuning and surface passivation and increasing pixel density using similarly simple and cheap patterning techniques. Due to the simplicity of the fabrication method used here this device structure and method has great flexibility. Bandgap selectivity can easily be extended to a greater number of QD sizes to give greater temperature fidelity. If the region between 4 and $5 \mu \mathrm{m}$ is of interest then only QDs with bandgaps in that region can be used. Finally it would be facile to extend this method to other materials i.e. a hyperspectral grid with LWIR, MIR, NIR, VIS and UV QDs on individual pixels. 
184 Chapter 5. Multi Pixel Imaging with Photogain Enhanced Detection 


\section{Chapter 6}

\section{Tin Chalcogenide Quantum Dots}

Parts of this work are published in:

Room Temperature MIR Detection through Localized Surface Vibrational States of SnTe Nanocrystals, Cryer, M. E.; Halpert, J. E. ACS Sensors, 2018.

\subsection{Motivation}

This work was motivated by the search for an MIR semiconductor QD that did not contain mercury or cadmium, as these are toxic materials that pose risks to health and the environment and are therefore unlikely to be suitable for mass market, consumer applications [221]. The size tunability of bandgap afforded by quantum confinement should allow, in a similar vein to HgTe, any bulk semimetal or small bandgap semiconductor to demonstrate tunable bandgaps throughout the IR. The more difficult part of this requirement is that films of these QDs should be electrically active in a simple, planar electrical structure. Fortunately, the solution processability of QDs, as seen throughout this thesis, allows easy device prototyping and ligand exchange testing. Thus the idea behind this work was to find colloids that offer MIR absorption and then use the methods from Chapters 
3 and 4 to test if these QDs offered measurable electrical behaviour with a simple post deposition ligand exchange. The Background Section below aims to give a brief perspective on non- $\mathrm{Hg}$ based QDs and, as SnTe based QDs were found to be the most promising candidate, a brief history of SnTe QDs. Due to the debatable nature of the MIR absorption feature, at points this work diverges from QD device behaviour into a spectroscopic study of what has commonly been reported as, but is not, QD band absorption.

\subsection{Background}

SnTe is relatively non-toxic, has a bulk bandgap of $0.18 \mathrm{eV}$ [222] and can be made into a zero bandgap material in the bulk, due to a bandgap crossing when alloyed with lead [223]. Additionally, the band structure of SnTe QDs is similar to HgTe QDs which have tuneable bandgaps in the MIR and LWIR [96], and would therefore appear to be an ideal candidate for a mercury free device. However, even with the numerous published reports of mercury-free, MIR absorbing CQDs [223-226] there are no reports of successful MIR photodetectors using these materials [226-229]. Similarly, quantum confinement [75] and band edge tuning in Hg-based materials remains the only aspect of QD physics that has been effectively utilised in MIR photodetectors [230]. Even though there have been many reports of QDs demonstrating other physical effects in the MIR, such as localised surface plasmon resonance (LSPR) [231-234] and surface interactions with adsorbed species [235-237], none of these have yet yielded a functioning optical device. The surface interface between neighbouring QDs is known to have a large influence on the conductance of QD thin films [23,78]. Yet the surface of MIR CQDs has always been treated as an area to passivate rather than utilise for detection. MIR absorption peaks have been shown in various reports of SnTe [226-229] and PbSnTe [223] QDs and have been variously ascribed to bandgap absorption [223,226], LSPR absorption [228] 
and ligand absorption [229]. Here we demonstrate that for films made from three sizes of SnTe QDs, as well as in PbSnTe QDs, there is an absorption peak that can be used to detect MIR photons due to changes in the resistance of the film. We are able to show that this is not a band edge effect because only IR light that is resonant with the surface absorption feature can be detected. Not only is this the first reported instance of MIR detection using SnTe CQDs but also represents the first device to use MIR QD surface species to sense incident MIR radiation though a simple 2terminal measurement of current. This effect cannot be seen in the bulk semiconductor or nanoparticles of tin oxide. Although PbSnTe QDs are more toxic than pure SnTe QDs they are included here as they represent one of the few published MIR absorbing QDs and, due to their differing band edge from SnTe, allow a greater understanding of the underlying mechanism.

\subsection{Experimental}

PbSnTe QDs were synthesised using a two-step hot injection method. In a typical synthesis $56 \mathrm{mg}$ of $\mathrm{PbCl}_{2}$ was dissolved in $12.5 \mathrm{ml}$ of oleylamine (OLA) and the mixture was then degassed under vacuum at $120{ }^{\circ} \mathrm{C}$ for $1 \mathrm{~h}$. The mixture was then placed under nitrogen and $0.16 \mathrm{ml}$ of bis[bis(trimethylsilyl)amino]tin(II) in $7.5 \mathrm{ml}$ of dry octadecene (ODE) was injected and the temperature raised to $150{ }^{\circ} \mathrm{C} .0 .73 \mathrm{ml}$ of a dry $1.0 \mathrm{M}$ trioctylphosphine:tellurium (TOP:Te) precursor was then injected and the mixture instantly turned black. The heat was removed, the mixture was left for $90 \mathrm{~s}$ and then quenched by submerging the vessel in ice water, followed by an injection of $3 \mathrm{ml}$ of oleic acid (OA). Once at $25{ }^{\circ} \mathrm{C}, 10 \mathrm{ml}$ of $1: 1$ chloroform:acetone is added and the mixture can then be either stored or processed. QD purification was performed in air by centrifuging with acetone/chloroform as the anti-solvent/solvent pair. PbTe QDs were synthesised using the same method without the $S n$ precursor. For 


\begin{tabular}{l|l|l|l|l|l} 
Sample & OLA 80 \% (ml) & ODE $(\mathrm{ml})$ & Sn $(\mathrm{mmol})$ & Te $(\mathrm{mmol})$ & $\mathbf{P b}(\mathrm{mmol})$ \\
\hline $9 \mathrm{~nm} \mathrm{SnTe}$ & 2.5 & 17.5 & 0.4 & 0.4 & - \\
$10 \mathrm{~nm} \mathrm{SnTe}$ & 5 & 15 & 0.4 & 0.4 & - \\
$12 \mathrm{~nm} \mathrm{SnTe}$ & 12.5 & 7.5 & 0.4 & 0.1 & - \\
$14 \mathrm{~nm} \mathrm{SnTe}$ & 15 & 5 & 0.4 & 0.4 & - \\
PbSnTe & 12.5 & 7.5 & 0.4 & 0.73 & 0.1 \\
Te NW soln & 1 & 19 & 0.4 & 0.4 & -
\end{tabular}

Table 6.1: Table showing the precursors used in the SnTe and PbSnTe QD syntheses.

$\mathrm{PbTe}$ the mixture does not turn black until $180{ }^{\circ} \mathrm{C}$ and was heated at this temperature for $20 \mathrm{~min}$ before quenching. SnTe QDs were synthesised using the same method without the $\mathrm{Pb}$ precursor. Differing sizes were achieved by varying the OLA concentration in the mixture. Exact details can be found in Table 6.1.

TEM samples were placed on Agar 300 mesh $\mathrm{Cu}$ on Formvar grids. Colloids were washed four times and dispersed in tetrachloroethylene (TCE) before being placed on the grids. Before imaging the grids were oxygen plasma cleaned for $30 \mathrm{~min}$. TEM, STEM and EDS measurements were done on a JEOL-2100 TEM. Absorbance measurements were done on a Bruker Tensor 27 FTIR with a diamond ATR attachment and a VISNIRMIR Bruker Vertex 80v. All film measurements were done on $1 \mathrm{~mm}$ thick $\mathrm{CaF}_{2}$ substrates purchased from Eskma Optics. QDs $\left(20 \mathrm{mg} \mathrm{ml}^{-1}\right)$ were spin coated onto substrates and devices at $1 \mathrm{~K} \mathrm{rpm}$ for $40 \mathrm{~s}$. In between layers the films were treated with a ligand exchange solution of $5 \mathrm{mg} \mathrm{As} \mathrm{As}_{3}$ in $30 \mathrm{ml}$ propylamine (PPA) and $30 \mathrm{ml}$ ethanol $(\mathrm{EtOH})$ and then rinsed in ethanol. Due to the low wetting angle of $\mathrm{CaF}_{2}$ compared to silane treated $\mathrm{SiO}_{2}$ the absorption of the $\mathrm{CaF}_{2}$ films provides a low estimate of the absorption of the film on the $\mathrm{SiO}_{2}$ devices. Device substrates were $\mathrm{Si} / \mathrm{SiO}_{2}$ wafers with $300 \mathrm{~nm}$ oxide layers. The first step in fabrication was surface treatment ((3-aminopropyl)trimethoxysilane in dry toluene for $30 \mathrm{~min})$ to deposit a silane self-assembled monolayer (SAM) and then $\mathrm{Cr} / \mathrm{Au}$ (5/50 $\mathrm{nm}$ ) inter-digitated electrodes (IDE) (36 fingers per side, $2 \mathrm{~mm} \times 20 \mu \mathrm{m}$ with 
$10 \mu \mathrm{m}$ spacing, device area is $4 \times 10^{-6} \mathrm{~m}^{2}$ ) networks were 1-step photolithographically deposited. All devices had drain source leakage currents below $10 \mathrm{pA}$ and gate source leakage below $1 \mathrm{nA}$ before QD films were deposited. Electrical measurements were completed on a Keithley SCS4200 parameter analyser with the device in ambient atmosphere in the sample chamber of an uncooled Janis VNF-100 optical cryostat. Both the mount and the chamber were monitored for temperature change during measurements. With optical filters in place the maximum recorded temperature change during measurement was $0.05 \mathrm{~K} \mathrm{~min}^{-1}$. The optical path for device illumination consisted of the cavity opening of an Omega BB$4 \mathrm{~A}$ blackbody (BB), $12 \mathrm{~cm}$ of air shielded from external light, the long pass filter, the chopper blade and two sapphire windows. Incident flux was calculated using a spectrally integrated plank function over the transmittance of the path and the effective solid angle obtained from calibration using a Thorlabs PM-100D with S401C power meter.

To remove any extant background effects the change in current was recorded for the $\mathrm{BB}$ being covered/exposed rather than the sample chamber being covered/exposed, which means that the measured current response is a result of the BB exposure, not exposure to any background level of high energy visible photons. Responsivity was calculated as the difference between the current during exposure and the current level to which the device returned once the IR radiation was removed, in order to avoid errors arising from film degradation.

To conclusively prove that some aspect of the SnTe QDs were responsible for the behaviour reported a number of tests were completed using the same ligand exchange and optical tests. Blank devices demonstrated no response. HgTe QD films treated with the same ligand behaved in a conventional photoconductive manner. Pure $\mathrm{SnO}_{2}$ nanoparticles (NPs) in powder form demonstrated similar conductivity to the SnTe QDs but showed no response to visible or IR light. Finally spin coated $\mathrm{SnO}_{2}$ dispersed in tetrahydrofuran (THF) had a resistance greater than $1 \mathrm{G} \Omega$ and 
no photoresponse to visible or IR light. Non IR illumination was provided by a Thorlabs $670 \mathrm{~nm}$ laser diode and any change in current upon illumination was less than the signal noise of the device. Current vs time measurements were taken with an effective sampling rate of $15 \mathrm{~ms}$ and a constantly applied bias. Temperature dependent conductivity measurements were taken with the same setup and filling the sample chamber with liquid nitrogen vapour to the required temperature. SEM images were taken on a JEOL 6500 SEM where before imaging the device was carbon coated to reduce charging. X-Ray diffraction patterns were recorded by a Panalytical $\mathrm{X}$-Ray diffractometer using $\mathrm{Cu}-\mathrm{K} \alpha$ radiation, with an operating voltage of $45 \mathrm{kV}$ and current of $40 \mathrm{~mA}$. The reference PDFs used were $\mathrm{PbO}_{2}$ 00-0411492, $\mathrm{SnO}_{2}$ 00-041-1445, SnTe 00-046-1210, PbTe 00-038-1435, $\mathrm{Pb}_{0.45} \mathrm{Sn}_{0.55} \mathrm{Te}$ 04-002-7237 and $\mathrm{Pb}_{0.1} \mathrm{Sn}_{0.9}$ Te 04-018-1386. ICP-MS samples were prepared by dissolving a solution of QDs in toluene after 4 washes, in $1 \mathrm{ml}$ nitric acid. This was then diluted by a factor of $10^{5}$ before measurement. XPS samples were prepared on pure Si substrates and treated with the same ligand exchange procedure as the devices.

\subsection{Results}

\subsubsection{XRD and Absorbance}

The samples were characterised initially with XRD to confirm the crystal structure. Example XRD data is shown in Figure 6.1 and confirms the cubic halite crystalline structure. 

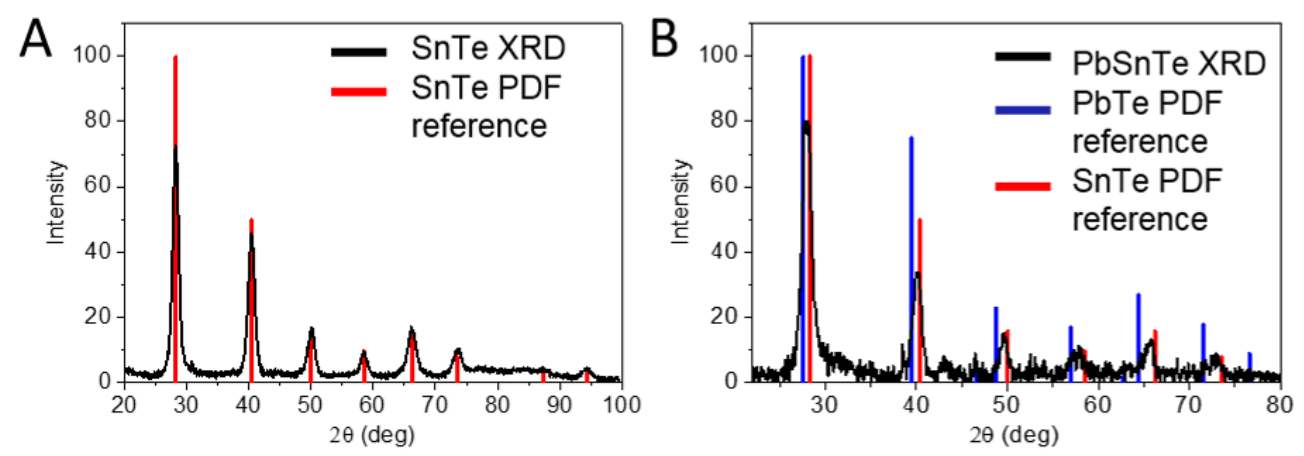

Figure 6.1: XRD data and reference for dried films of the A, $12 \mathrm{~nm}$ SnTe and B, PbSnTe $Q D s$, the PbSnTe peaks sit between the reference spectra of SnTe and PbTe. There are no peaks attributable to oxides in the XRD data. Indicating that the majority of the samples here are semiconductor lattice.

To determine if the various samples showed MIR absorbance they were looked at with ATR absorption measurements. For the samples that were subsequently used in devices the initial ATR results are shown in Figure 6.2. These spectra are similar to those previously reported in the literature for these materials [223,226-229]. The largest SnTe QDs at $13.6 \mathrm{~nm}$ did not make reliable devices but are included to show the band edge progression with size. As well as what looks like a band edge there is also a very broad absorption peak centred around $3125 \mathrm{~cm}^{-1}$ (shown by the red dashed line at $3125 \mathrm{~nm}$, the peak stretches from $2700 \mathrm{~nm}$ to $\approx 5000 \mathrm{~nm}$ ). This feature does not vary in position with either reaction condition or QD size, this is highlighted here as it is this feature that is discussed in the rest of this Chapter. In these materials, as will be shown, it is not possible to state an accurate bandgap value due to the absorption spectra being due to a number of features. Though it may appear at first inspection that these are just highly disperse MIR active QDs similar to HgTe, this is not the case as will be shown. 


\section{Wavelength $(\mu \mathrm{m})$}

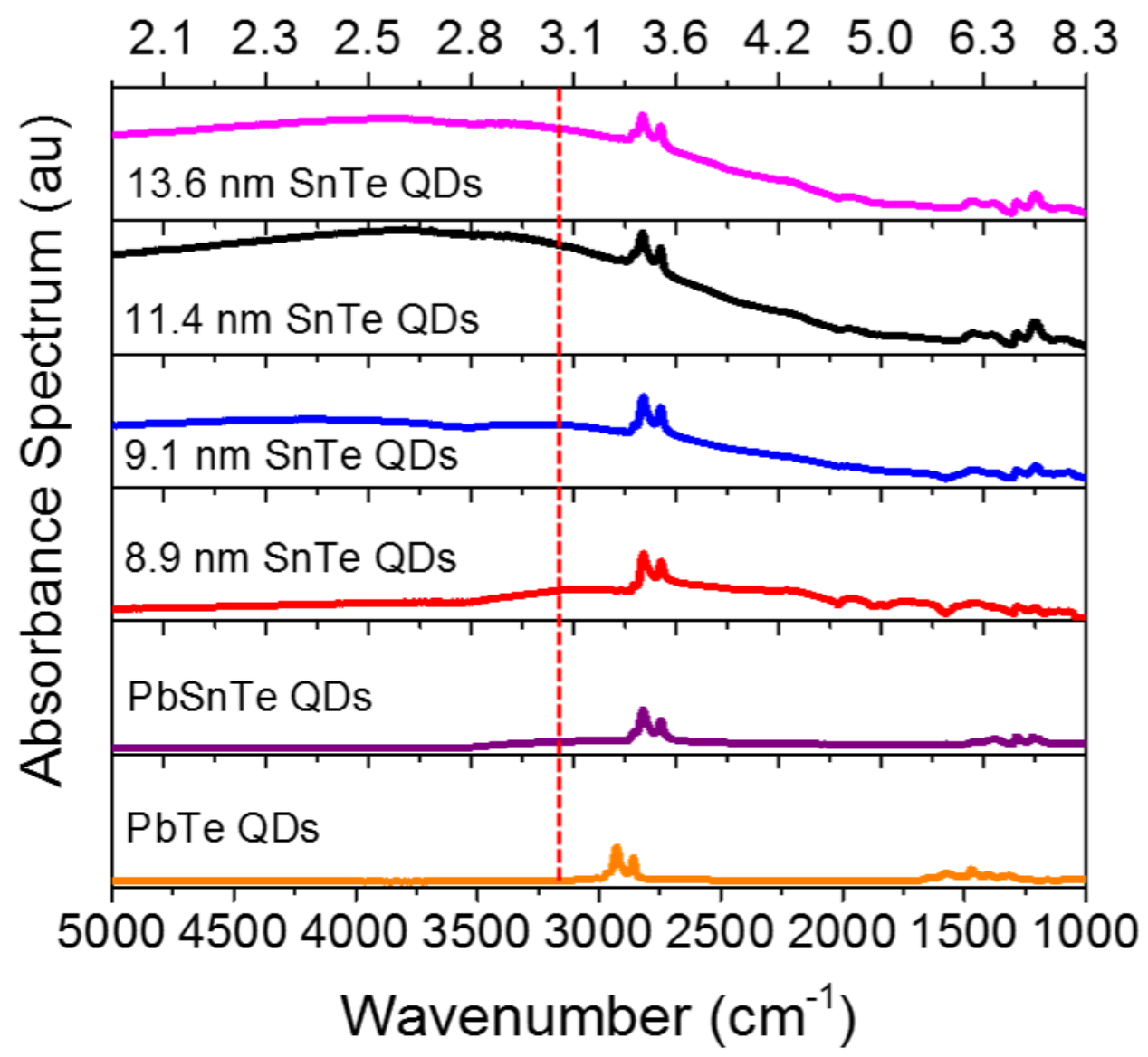

Figure 6.2: Absorption of the CQD solutions as synthesised, drop-cast and dried on the ATR module of an FTIR. There is a clear absorption at $2.95 \mu \mathrm{m}$, except in the PbTe samples. This is marked with the red dashed line.

Films of the colloids were successfully ligand exchanged with the infrared inactive material $\mathrm{As}_{2} \mathrm{~S}_{3}$ to increase the conductivity of the thin film. As the QDs are predominantly OA capped following synthesis a number of more common ligand exchanges were attempted, including ethandithiol, ethanediamine and formic acid, however none of these yielded increases in film conductivity. Figure 6.3 A shows the increase in 
conduction for the same sample before and after the inorganic exchange, giving an increase of three orders of magnitude to the measured current. Fig 6.3 B shows the film absorbance for a typical (although not strongly absorbing) spin cast SnTe film on $\mathrm{CaF}_{2}$, under three post treatment conditions. As absorbance will be heavily discussed in this Chapter
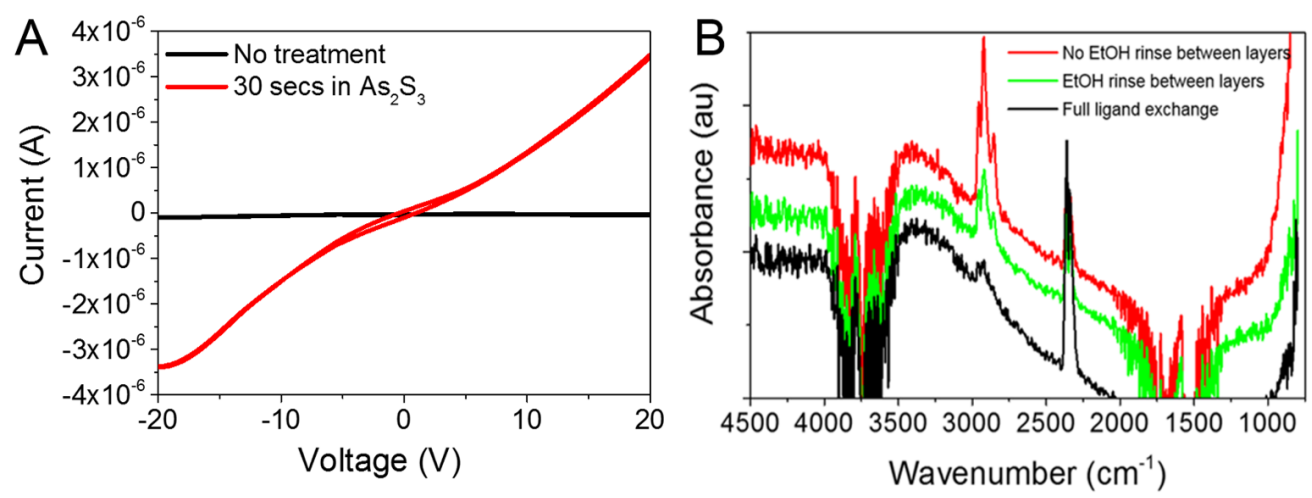

Figure 6.3: $A, I V$ curve of example SnTe device before and after the ligand exchange. $B$, Film absorbance of SnTe films (identical in all other respects) made with different processes, vertically separated for clarity, described at length in the text.

it is instructive to explain the dominant features in Figure 6.3 B. The noise between 4000 and $3500 \mathrm{~cm}^{-1}$ is due to atmospheric $\mathrm{O}-\mathrm{H}$ sretching in the beam path, noting that atmospheric $\mathrm{H}_{2} \mathrm{O}$ absorbs at a different wavenumber than liquid water. The feature centred at $\approx 3200 \mathrm{~cm}^{-1}$ is the feature of interest and is discussed at length throughout this Chapter. The C-H stretch is seen at $2900 \mathrm{~cm}^{-1}$ and is due to the ligands and any other non surface bound organics that remain in the film. The feature at 2400 $\mathrm{cm}^{-1}$ is the $\mathrm{C}-\mathrm{O}$ stretch of atmospheric $\mathrm{CO}_{2}$ in the beam path. The noise centred at $1650 \mathrm{~cm}^{-1}$ is atmospheric $\mathrm{O}-\mathrm{H}$ bend and the feature below 800 $\mathrm{cm}^{-1}$ is attributed to the $\mathrm{Sn}-\mathrm{O}$ stretch of oxidised surface Sn lattice sites, although the SnTe lattice phonon is also likely to absorb in this region.

Each line in Figure 6.3 B represents a film made from an identical (same substrate, same colloid, same volume, same ramp rate etc) single spin coating step, followed by one of three treatments, as indicated. There is a clear 
reduction in the organics when just using the $\mathrm{EtOH}$ rinse (green line compared to red line) indicating that the EtOH is definitely removing organics. Without further, very detailed work it is not possible to say whether these are just excess organics remaining from synthesis, or if ligands have also been removed. However, as there is a further reduction with the ligand exchange step (black line compared to green line), which is also partnered by the conduction increase, it is likely that at least a portion of the ligands remain after the $\mathrm{EtOH}$ rinse and these are substantially reduced further by replacement with $\mathrm{As}_{2} \mathrm{~S}_{3}$.

The near-infrared (NIR)-MIR absorbance of the films (normalised at $12000 \mathrm{~cm}^{-1}$ ) is shown in Figure 6.4. In this plot the absorbance feature at $3200 \mathrm{~cm}^{-1}$ is present in all samples and the band edge of the QD films is also clearly visible. Importantly the band edge of the PbSnTe film drops to zero before $4000 \mathrm{~cm}^{-1}$ and it can therefore be seen that the feature at $3200 \mathrm{~cm}^{-1}$ is separate to the band edge absorption of the nanocrystal. Additionally, the pseudo-excitonic peak in the SnTe QD films is in fact a juxtaposition of the $3200 \mathrm{~cm}^{-1}$ feature and the monotonically decreasing band edge, rather than solely representing the band edge excitonic states of a highly confined QD (as is seen in the HgTe absorption in Chapter 3). Considering the size dispersions of these colloids (see the TEM images and sizing below) it would not be expected than an excitonic peak is visible. Certainly it would not be at exactly the same position (red dashed line in Figures 6.2 and 6.4) for all the sizes of QD. Additionally it is very important to indicate that in Figure 6.2 each plot shares the same $\mathrm{y}$-scale, and therefore it can be seen that the SnTe band edge absorption is, relatively, a lot stronger than the feature absorption. 


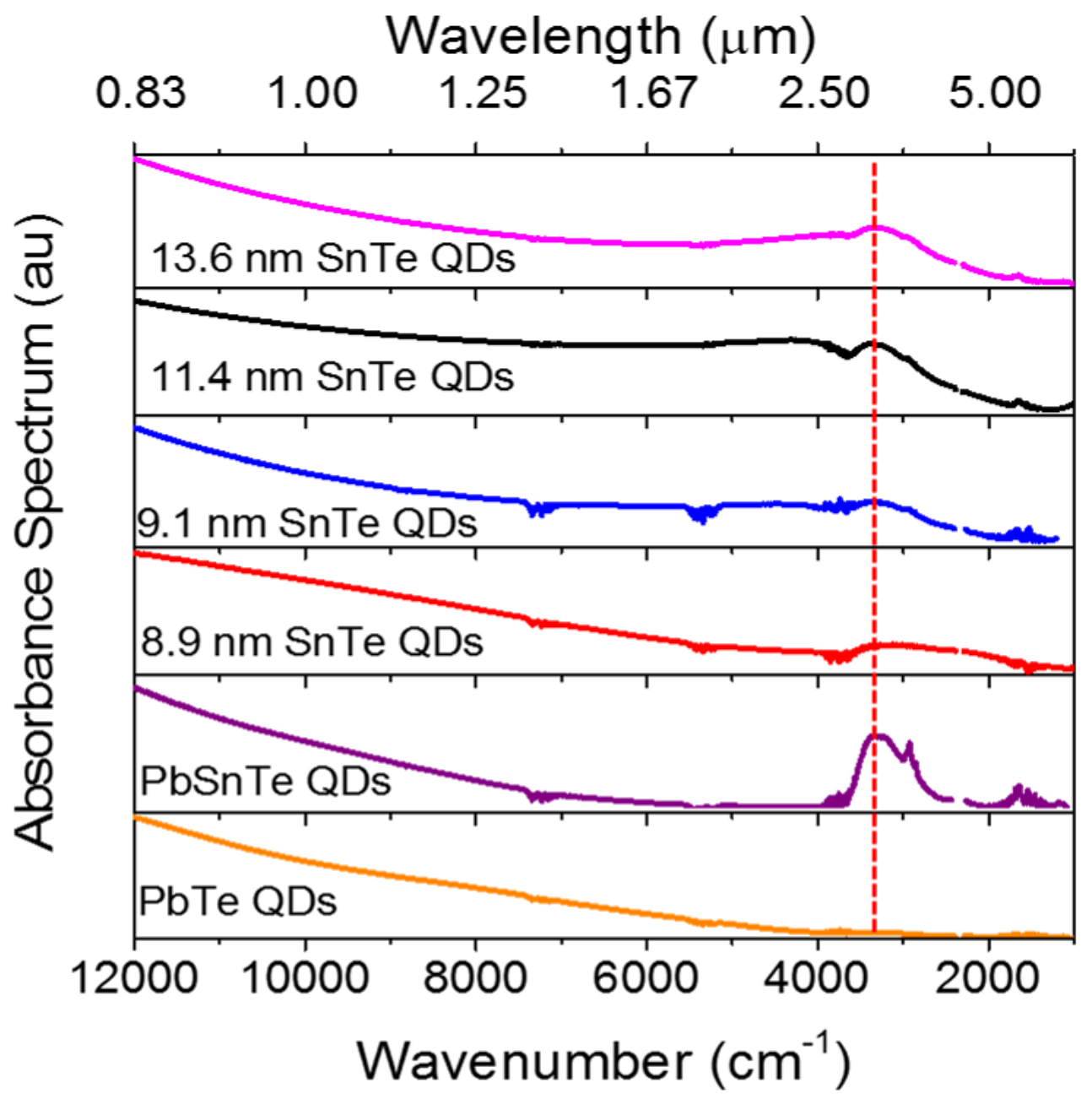

Figure 6.4: NIR-MIR absorption of ligand exchange treated films on $\mathrm{CaF}_{2}$ substrates. Normalised to the absorption at $12000 \mathrm{~cm}^{-1}$, all measurements were done in air and the $\mathrm{CO}_{2}$ absorption at $2400 \mathrm{~cm}^{-1}$ has been removed. The remaining noise areas are due to atmospheric $\mathrm{H}_{2} \mathrm{O}$ in the optical path. There is a clear absorption at $2.95 \mu \mathrm{m}$, except in the PbTe samples, this is marked with the red dashed line.

\subsubsection{QD Characterisation}

In the TEM images (Figures 6.5 to 6.9) SnTe and PbSnTe lattice fringes are clearly evident and show lattice spacing that is attributed to the halite structure observed by XRD. It is expected that unpassivated Sn surfaces 
would quickly oxidise in air [238]. However, there are no tin oxide peaks visible in the XRD data (Figure 6.1) so oxidation is presumed to be limited to a small region at the surface, rather than individual $\mathrm{SnO}_{2} \mathrm{NPs}$ or other large crystalline volumes.

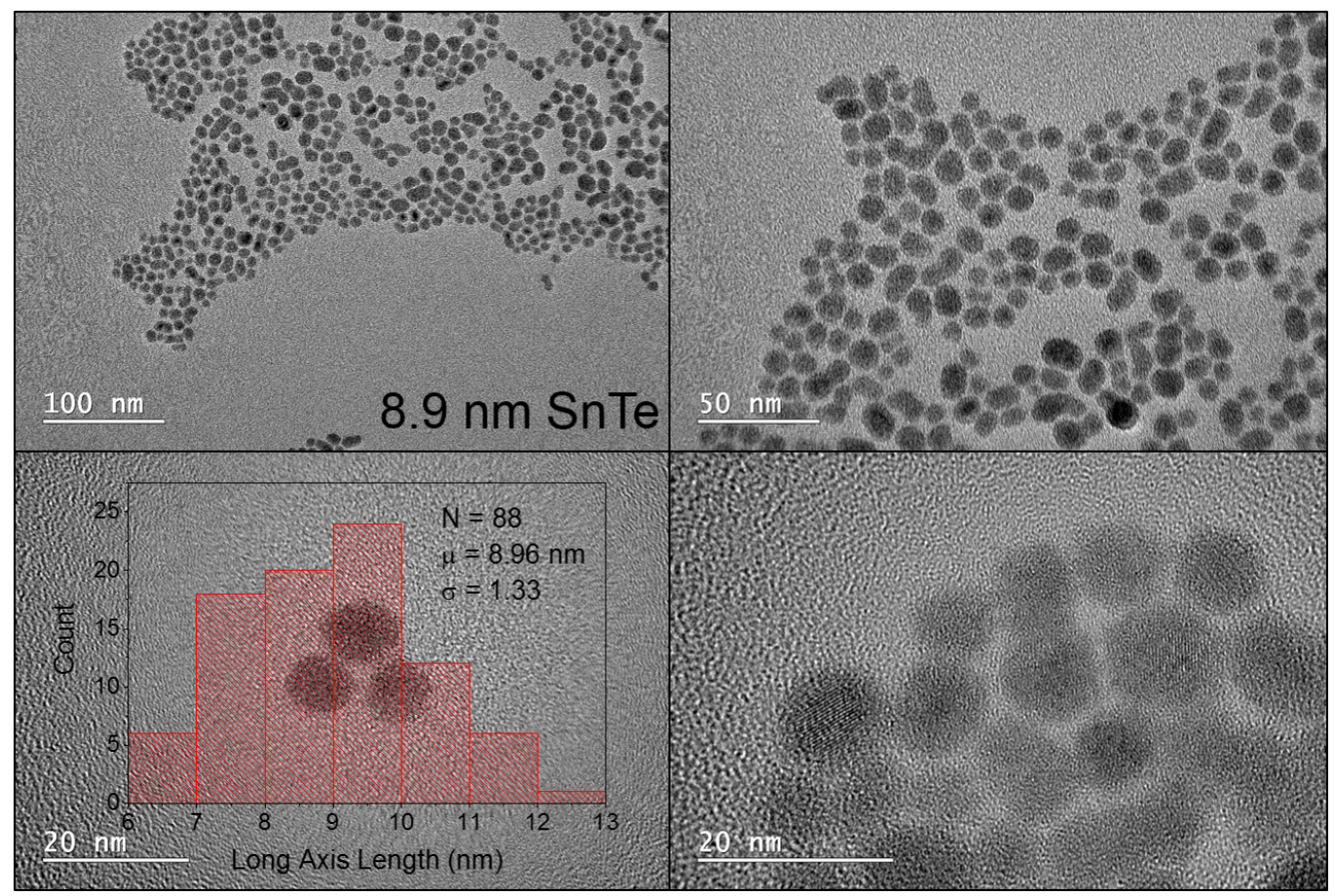

Figure 6.5: TEM images at various magnifications of the smallest sample that made functioning devices. Note the large size dispersion that would indicate a long Urbach tail, causing an ill-defined band edge and little excitonic featuring visible in the absorption spectra. 


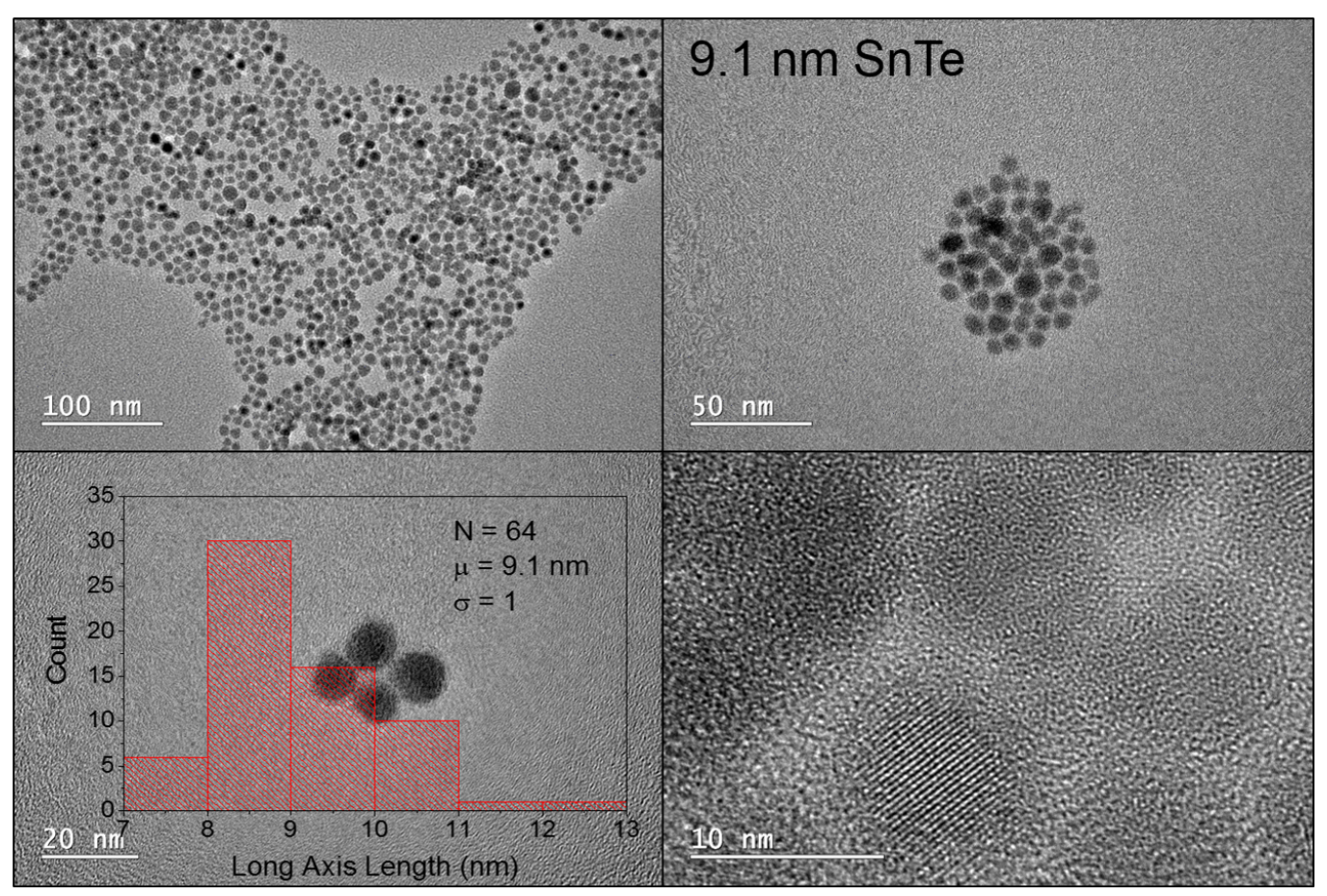

Figure 6.6: TEM images at various magnifications of the $9.1 \mathrm{~nm}$ samples. The crystalline core with some shell materials is visible in the bottom right. 


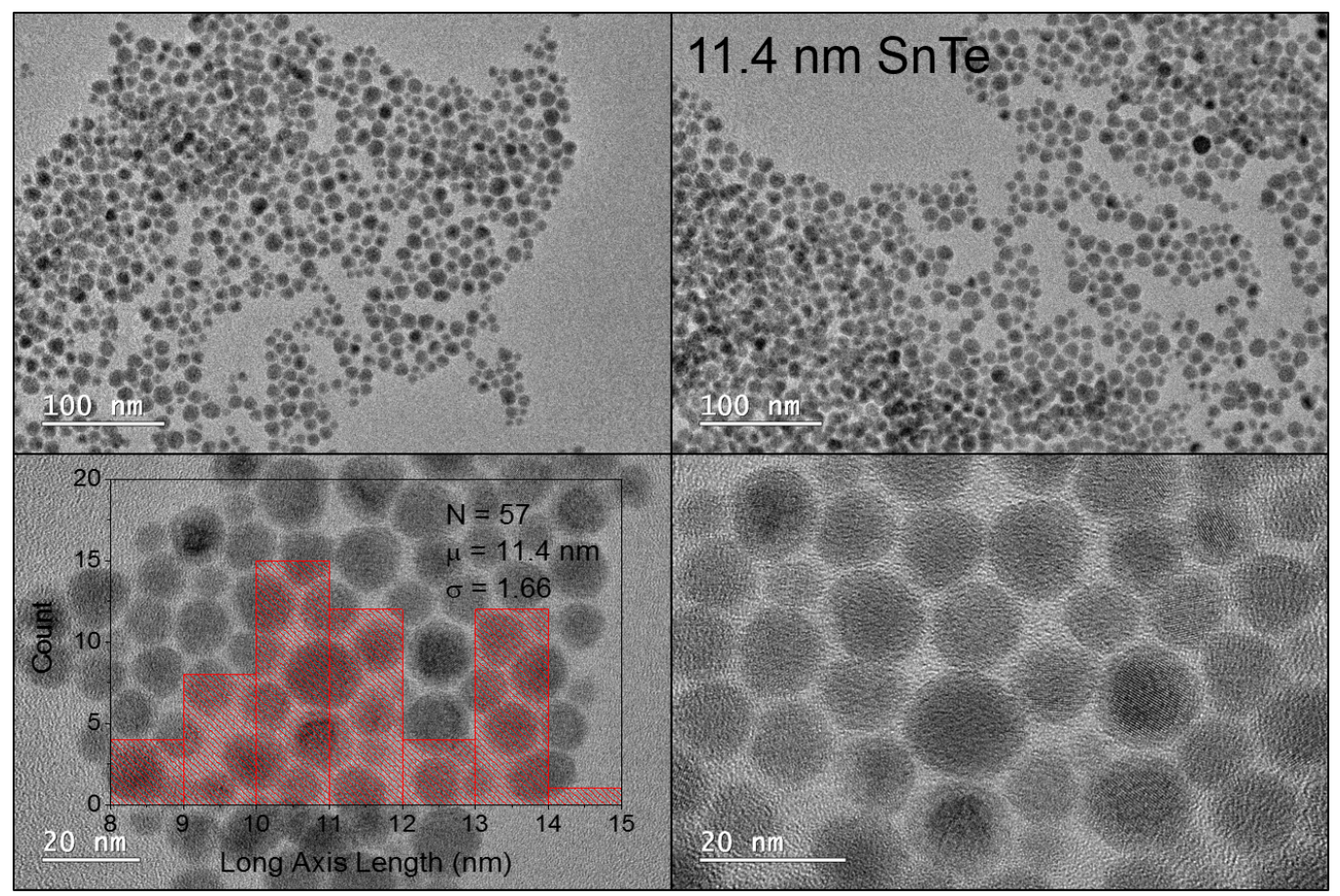

Figure 6.7: TEM images at various magnifications of the $11.4 \mathrm{~nm}$ samples. 


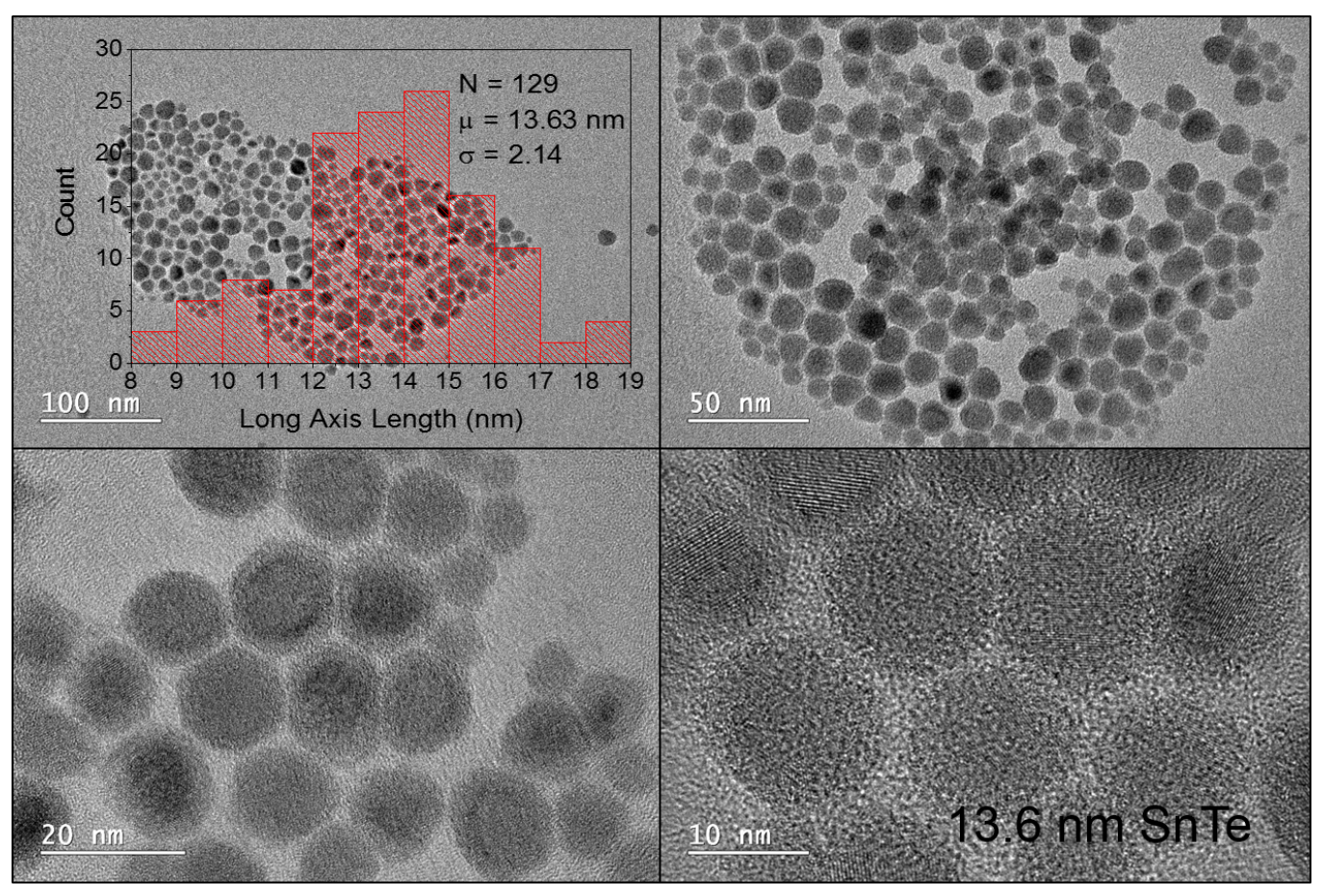

Figure 6.8: TEM images at various magnifications of the $13.6 \mathrm{~nm}$ samples. These QDs could not be fabricated into electrically stable devices. The core/shell structuring is visible in the bottom left. 


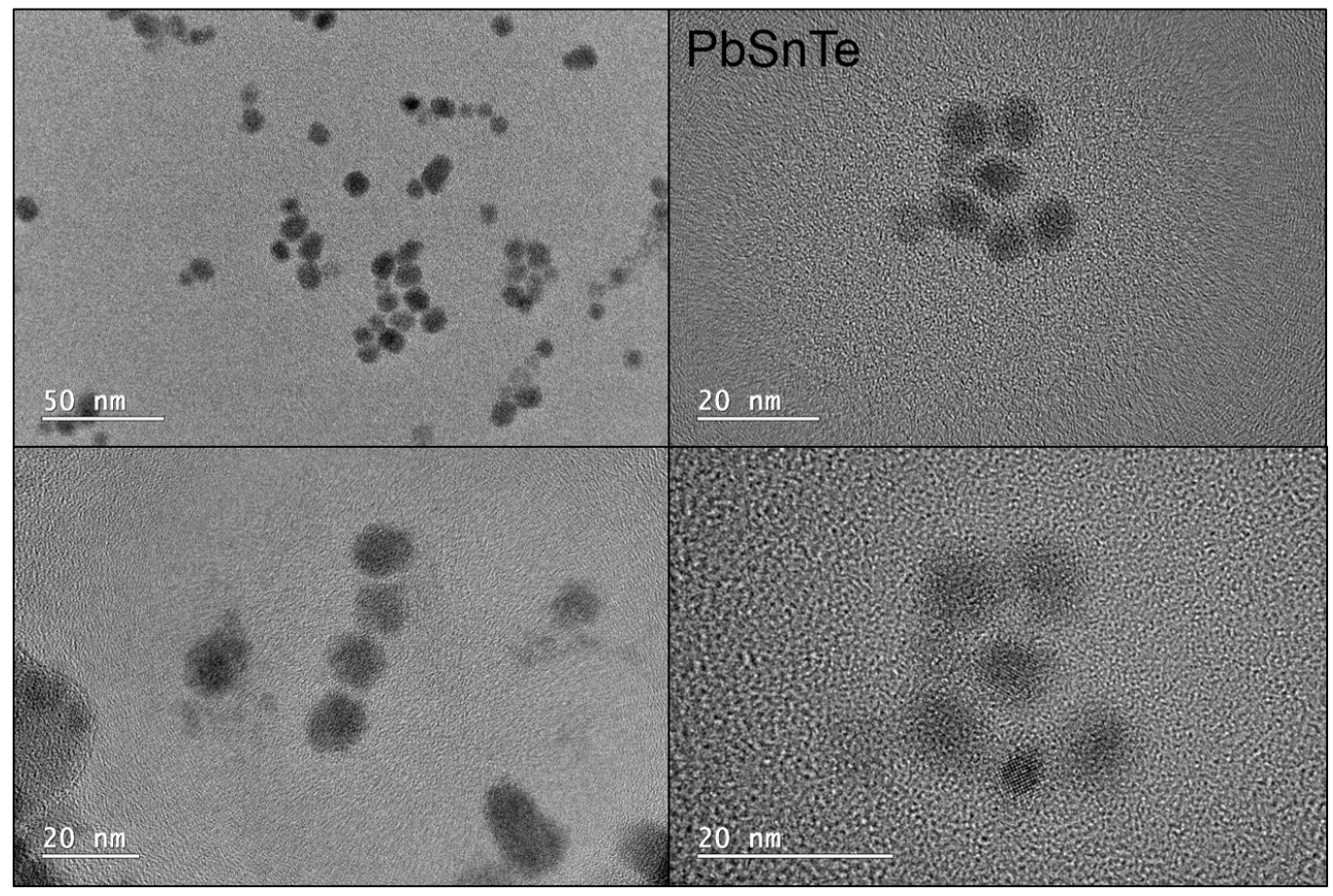

Figure 6.9: TEM images at various magnifications of the PbSnTe sample that was used in the devices reported here. 
The TEM images frequently show a shell region with discontinuous lattice fringes, indicative of an amorphous surface, examples of which are shown in Figure 6.10. For context these images can be compared with those of HgTe in Chapter 3 to see what lattice fringes look like for a QD with a complete crystalline structure. To be clear, in this context core/shell means that the observed physical structure in TEM imaging has two distinct regions, a core region and a shell region. It is not commenting on the energy levels of the material.

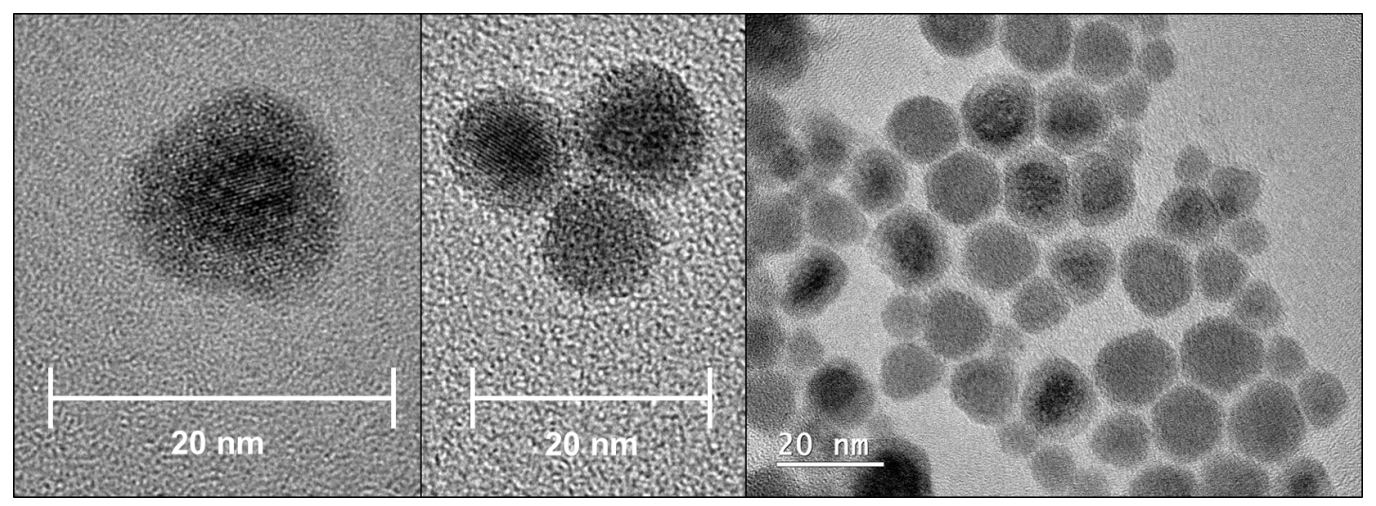

Figure 6.10: TEM images showing very clear examples of $Q D$ s that have a crystalline discontinuity in their structure.

Continued investigation with STEM EDS mapping (shown in Figure 6.11 and Figure 6.12) confirms that all of the intended precursors are localised to the QDs, indicating that TEM EDS measurements are accurately measuring QD composition and not including some background value for one of the elements of interest. Despite much effort allocated to imaging, the shell region was never sufficiently distinguishable in EDS mapping to the same degree that it was observable in standard TEM. Guo et al. showed explicitly that SnTe nanocubes oxidise at their surface, and were able to explicitly map this with STEM, which can be seen in Figure 3 of reference [228]. Those materials also showed absorption features similar to that shown here. 


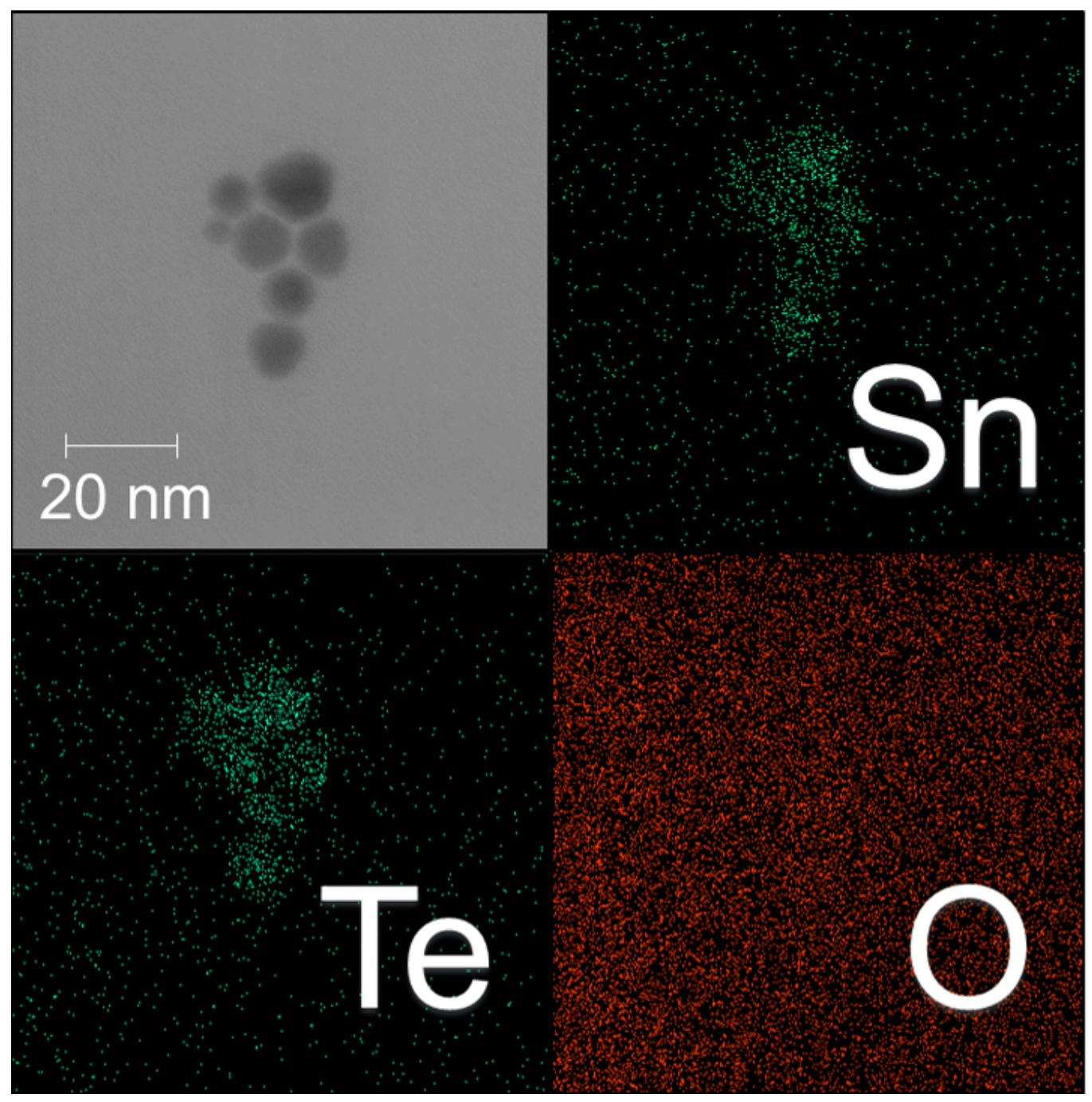

Figure 6.11: STEM mapping for $11.4 \mathrm{~nm}$ sample, showing the localisation of the elements that are being measured with EDS. The oxygen plot is included to demonstrate that any oxide layer here is not sufficient to be detected above the background on the grid. 


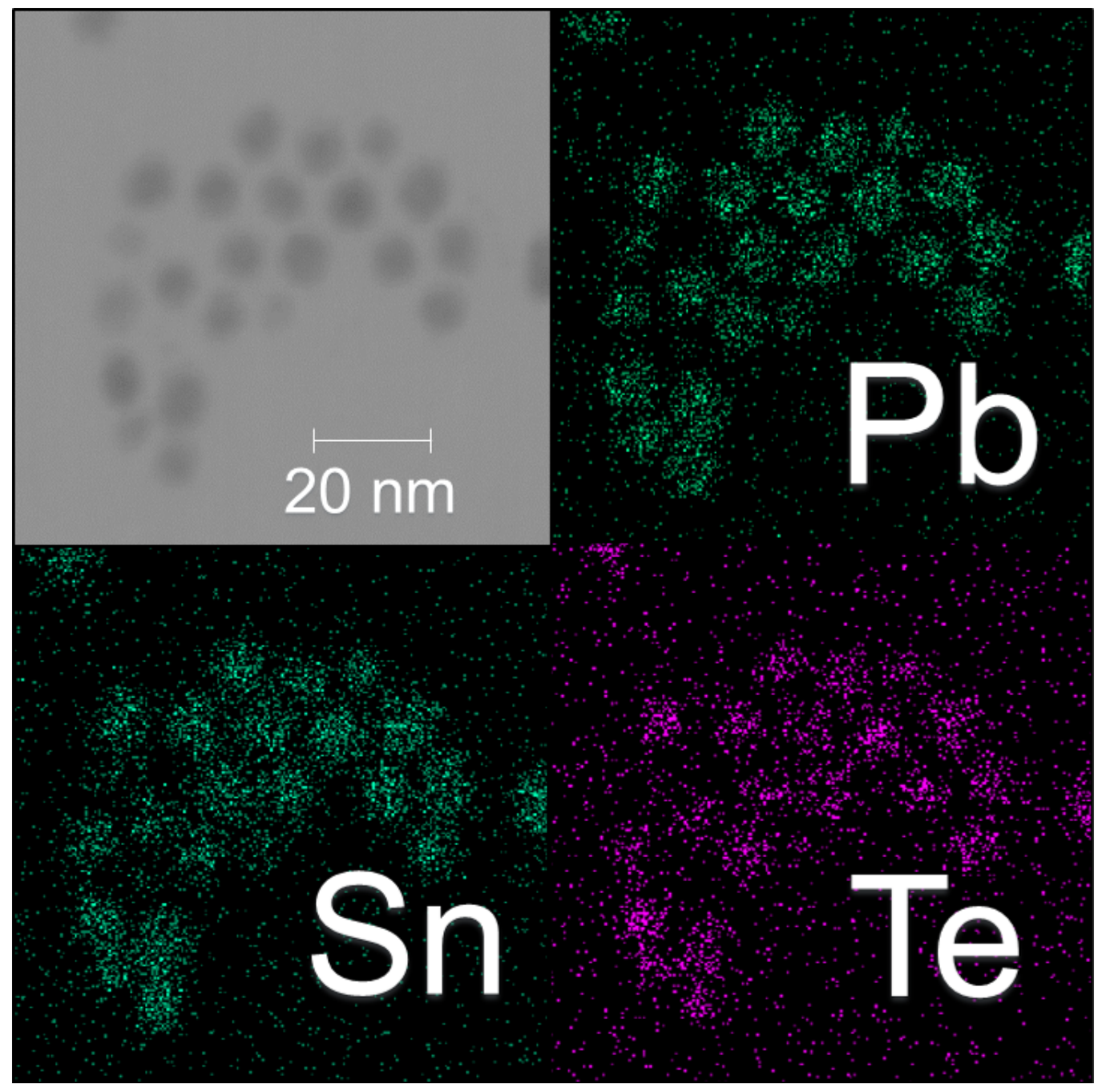

Figure 6.12: STEM mapping for PbSnTe, showing the localisation of the elements that are being measured with EDS. The Te deficiency is qualitatively visible in the Te map. 
TEM EDS shows a consistent stoichimetric Te deficiency, and this was confirmed by ICP-MS measurements. A comparison between these two compositional measurements is shown in Table 6.2. There is consistent discrepancy with the ICP-MS data giving higher Te values (lower ratios) than the TEM EDS. ICP-MS samples were run for a large number of samples and this difference remained. The constant higher quantity of Te found in the ICP-MS samples is attributed to the oxygen displaced Te physically remaining solution that was then diluted into the liquid samples run through the ICP-MS. Comparatively, the TEM EDS samples were targeted on areas of high QD density so would not include remaining Te precursor in the same ratio. All of which is highly indicative of a semiconductor QD with a $\mathrm{Sn}$ rich surface region where the $\mathrm{Sn}$ has oxidised, forming $\mathrm{SnO}_{2}$. This hypothesis was later directly confirmed by XPS measurements (Figure 6.13) which showed that SnTe QDs returned a dominant Sn oxidation state of +4 , consistent with $\mathrm{SnO}_{2}$, with a slight amount of +2 which is the SnTe lattice state. Conversely the Te oxidation state in SnTe samples was -2 which is consistent with the SnTe lattice only [239]. In short, at the surface of the NCs the Sn is mostly oxidised but the Te in the same depth region is still part of the lattice.

\begin{tabular}{l|l|l|l|l|l|l} 
& \multicolumn{2}{|l|}{ TEM EDS Atomic Ratio } & \multicolumn{2}{l|}{$\begin{array}{l}\text { ICP-MS Atomic Ratio } \\
\text { Sn:Te }\end{array}$} & $\begin{array}{l}\text { Preparation } \\
\text { OLA 80 \% (ml) }\end{array}$ & ODE \\
\hline SN1 & 1.06 & N/A & 0.84 & N/A & 5 & 15 \\
SN2 & 1.78 & N/A & 1.53 & N/A & 7.5 & 12.5 \\
SN3 & 1.76 & N/A & 1.56 & N/A & 10 & 10 \\
PN1 & 2.83 & 3.25 & 2.73 & 3.19 & 12.5 & 7.5
\end{tabular}

Table 6.2: Table showing TEM EDS and ICP-MS atomic ratios for 4 samples. 


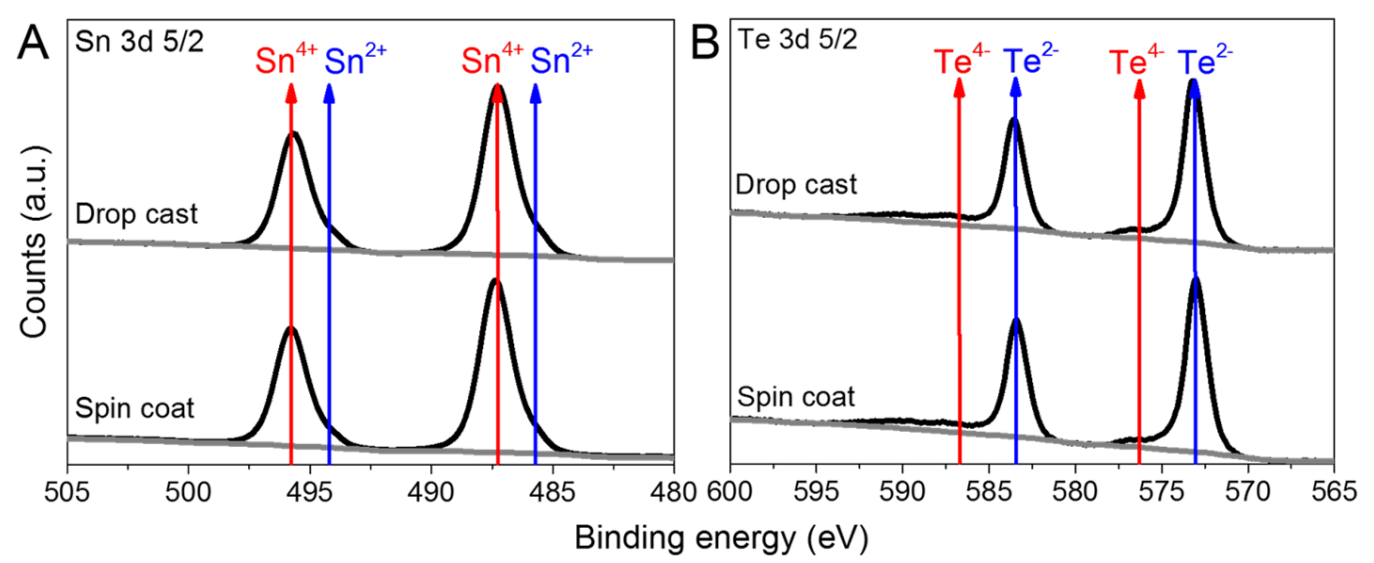

Figure 6.13: A, XPS data for the Sn oxidation state. B, XPS data for the Te oxidation state. Drop cast samples were used in addition to spin coated samples in order to ensure that there were sufficient QDs on the sample. All samples were treated with the same ligand exchange procedure as all the devices and films reported in this Chapter.

\subsubsection{Device Characterisation}

For electrical measurements the QDs were spin-coated onto gold interdigitated electrode (IDE) structures on $\mathrm{Si} / \mathrm{SiO}_{2}$, and then ligand exchanged producing an even film. SEM images of a SnTe device are shown in Figure 6.14 A, and SEM EDS maps of the same image are shown in Figure 6.14 B. The EDS mapping shows that the films have covered the devices evenly. The oxygen map offers no evidence with regard to film oxidation as there is a layer of oxide directly beneath it. Due to the unprecedented behaviour reported here, a large number of control devices were made, sensitised with: no QDs, $\mathrm{SnO}_{2}$ NPs and HgTe QDs. All were investigated with and without ligand exchange. None of the control devices showed behaviour similar to the Sn-based QD devices. The HgTe devices were photosensitive to the MIR, but these showed the expected normal photoconductive behaviour. 


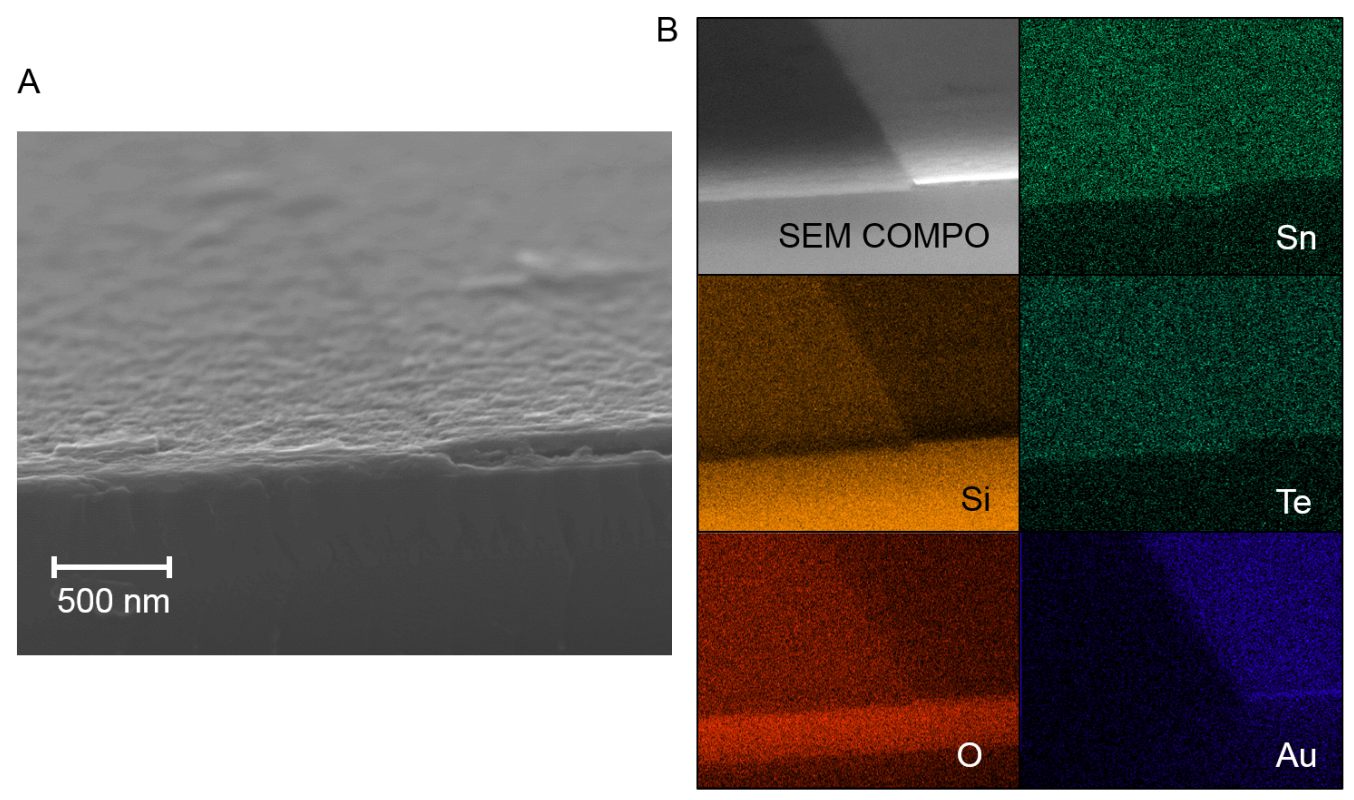

Figure 6.14: A, SEM image of the both the surface and cross section of an SnTe device. Note the even film coverage, the raised feature to the right of the image is one of the Au electrodes. B, EDS mapping of the same area. The Sn and Te are evenly spread across the surface, any oxidation in the film is obscured by the oxide layer beneath.

\subsubsection{MIR Optical Response}

Figure 6.15 shows a typical set of IV curves for the SnTe and PbSnTe QD devices, with and without MIR radiation, operated at room temperature in air. None of the devices displayed a photoresponse to visible light. However, when exposed to a broadband IR source generated by a BB source the films demonstrated a perfectly time correlated decrease in conductivity. 


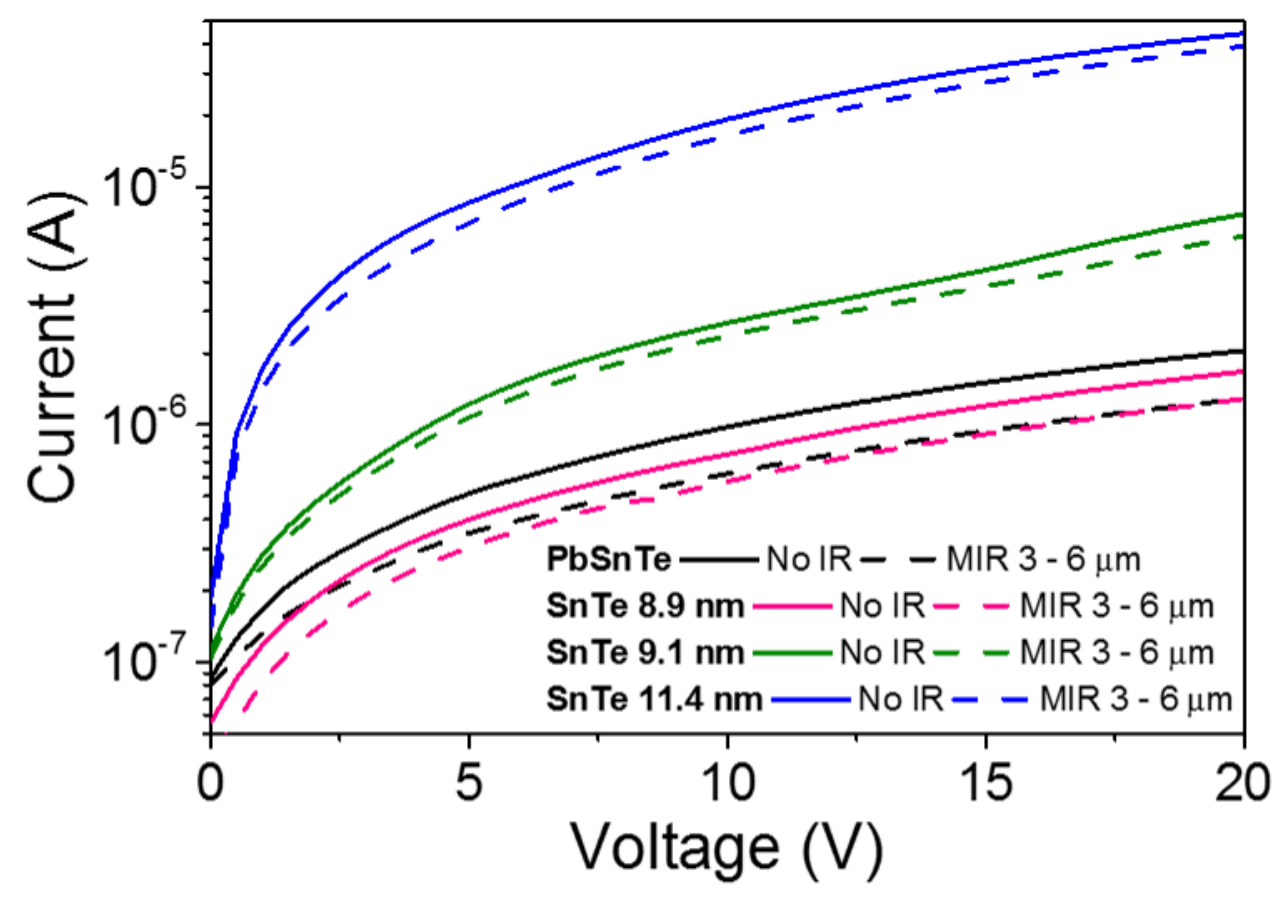

Figure 6.15: All of the devices show a reduction in conductivity when exposed to MIR illumination in a controlled setting where the only variable is the presence of an MIR source. The solid line is measured after the dashed line in all cases. None of these samples showed a measurable change in conductivity when exposed to visible light of any wavelength or intensity.

The magnitude of the conductivity decrease was proportional to the intensity of the incident radiation between 2.8 and $5 \mu \mathrm{m}$. When the IR was removed the conductivity returned to the original level. The incident flux is controlled via longpass filters that spectrally cut the incident light and as a result also modulate the total incident power. This confirms that there is both a proportional electrical response to the incident light and more importantly, that it is observed when only MIR photons are present, as shown in Figure 6.16. 


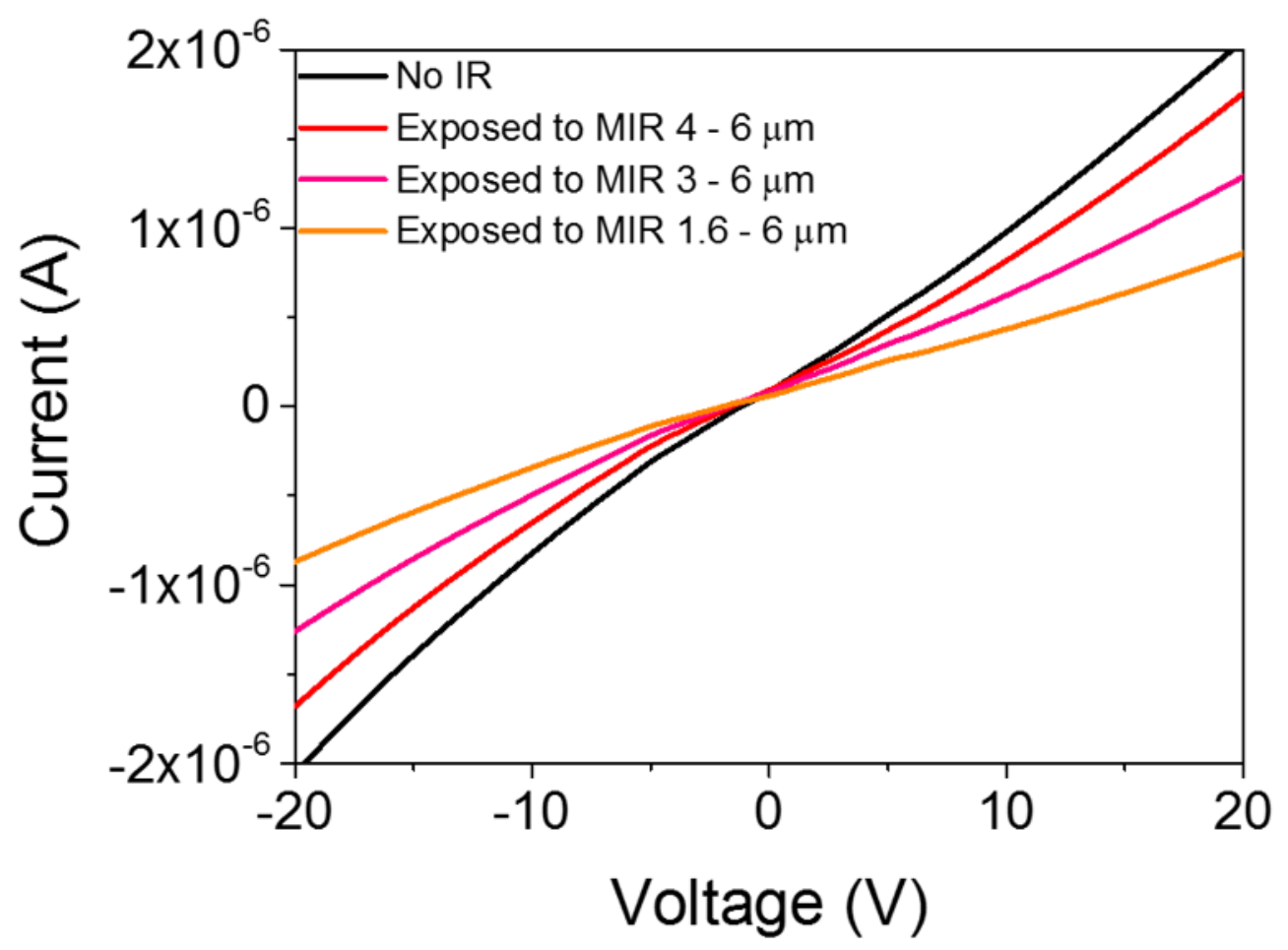

Figure 6.16: The current flowing through the device is inversely related to the intensity of the IR illumination, as the BB source is broadband the total intensity can be modulated by spectrally cutting the source. These measurements are taken with the current increasing, i.e. the largest IR flux measurement, resulting in the largest reduction in current is taken first and then the current recovers as the incident IR flux is reduced.

Figure 6.17 A shows the responsivity versus bias for each QD sample to incident BB spectra with a maximum photon energy of $410 \mathrm{meV}$, with a $10 \mathrm{~s}$ settle time. The highest responsivity was found for the $11.4 \mathrm{~nm} \mathrm{SnTe}$ QD device, with a value of $2 \mathrm{~mA} \mathrm{~W}^{-1}$ at wavelengths above $3 \mu \mathrm{m}$, at room temperature, with $5 \mathrm{~V}$ bias. Figure $6.17 \mathrm{~B}$ shows the decrease of current proportionally to the dark current (which in these devices is the higher value). 

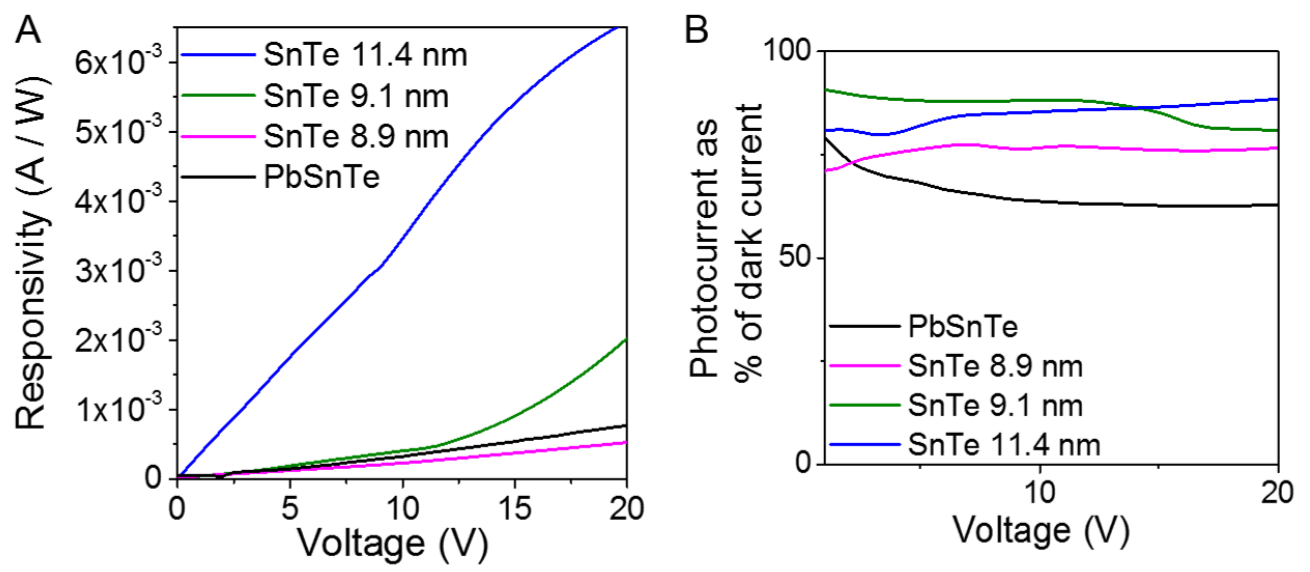

Figure 6.17: A, the performance of the devices as defined by the responsivity as a function of voltage for illumination with a 3-6 $\mu \mathrm{m}$ MIR source. B, the photocurrent varying with voltage used to calculate the responsivity in A shown as a percentage of the dark current in each device. This plot is showing how each device had its dark current reduced, relatively, by exposure to the 3-6 $\mu \mathrm{m}$ MIR source.
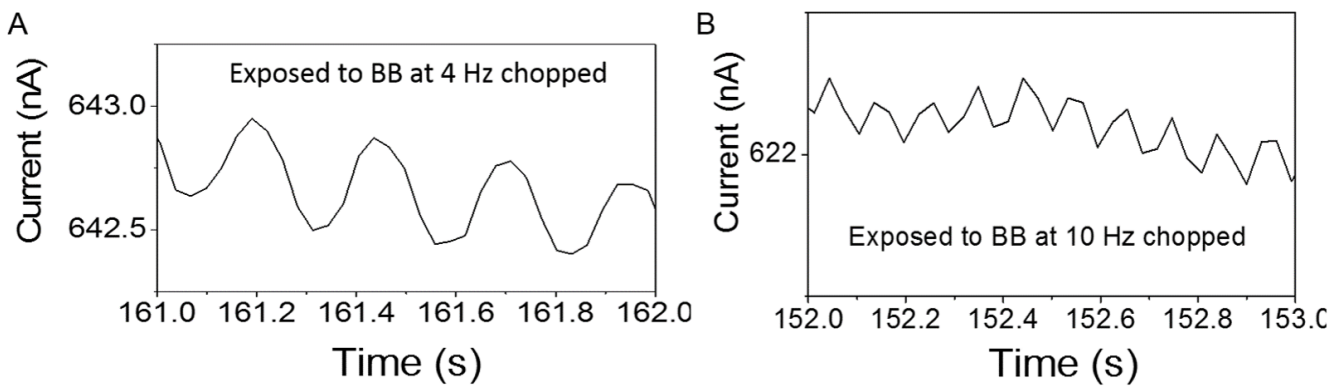

Figure 6.18: Both plots are for the same PbSnTe based device. A, current time plot for $B B$ source chopped at $4 \mathrm{~Hz}$. B, current time plot for BB source chopped at $10 \mathrm{~Hz}$.

Figure 6.18 shows the current response to the MIR source when the 3-6 $\mu \mathrm{m}$ signal from a $1073 \mathrm{~K} \mathrm{BB}$ is chopped at $4 \mathrm{~Hz}$ and $10 \mathrm{~Hz}$. The response time of these devices is slow compared to the best HgTe sensors [205] but better than that reported for HgSe devices [112]. From a performance perspective, an inverse photoresponse has a limited specific detectivity, due to its inherently high noise level. The mechanism and the zero current response to visible light shown here offer valuable insight into QD film 
physics and perhaps suggest a way to produce novel light and even analyte sensors using surface species. As such, and as this is the first report of this behaviour, it is worth exploring in depth.

\subsection{Discussion}

Any discussion of the mechanism in these devices must address two key issues, the MIR absorption feature and the inverse photoresponse of the devices. As is shown, the photoresponse only occurs for IR light that overlaps the absorption feature around $3200 \mathrm{~cm}^{-1}$, and not for light sources at higher energies $(\lambda<2 \mu \mathrm{m})$. The mechanism is therefore almost certainly related to a resonant effect in the MIR. In the case of band absorption, the features would be expected to vary with QD composition, shape and size and cause an increase of carriers with excitation, not a decrease. This limits the mechanism to either LSPR or vibrational excitation. There have been several previous reports of MIR features in this region [112, 231,240,241], however further involved discussion of this topic is warranted given the device behaviour observed here.

\subsubsection{Absorbance Features}

It has been suggested that this feature is an LSPR [228], but if that were so it must change energy as the QD size and composition varies [231]. As well as noting the large variation in size and composition of the QDs shown here, these MIR absorption features have also been reported in various forms of SnTe QDs that are reported as being both Sn deficient [227] and Sn rich [228]. This strongly implies that the feature is a result of Sn-related surface chemistry rather than an LSPR or intraband state. The PbTe QDs showed no such MIR absorption [242], but were prepared with the same organic ligands, discounting the oleic acid terminal group as the cause of the static absorption feature. It is well established, and shown again here 
with the XPS measurements, that any unpassivated surface Sn sites of SnTe are quick to oxidise [238]. Here we suggest that the absorption feature is the result of an adsorbed hydroxyl group on the oxidised QD surface of Sn containing QDs, a well understood feature reported many times previously [237,243-246]. This oxidation occurs at poorly passivated regions of the QD surface from trace oxygen introduced during the synthesis or during processing [242,247].
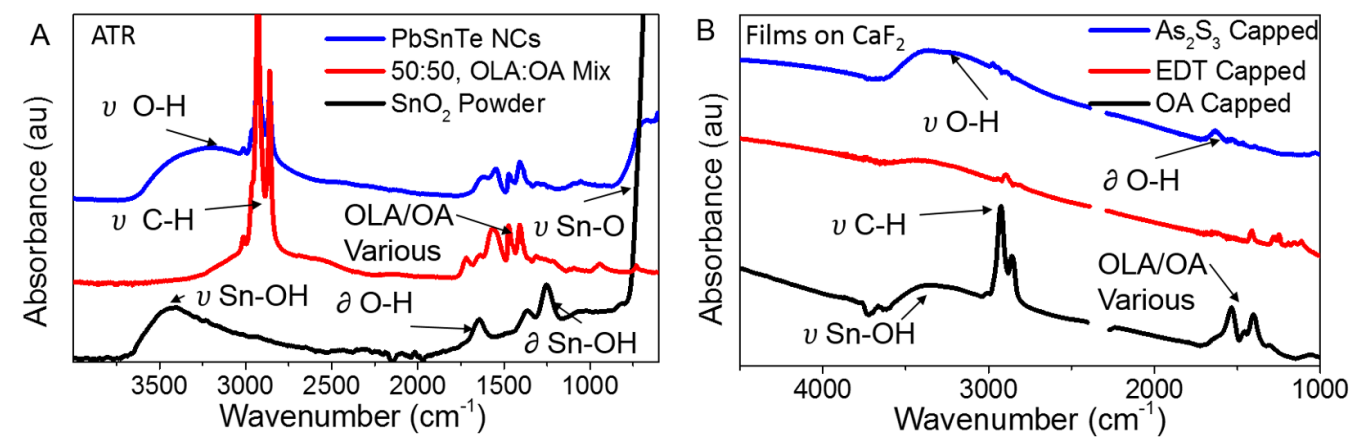

Figure 6.19: $A$, ATR spectra, y-separated, of drop-cast PbSnTe QDs with pure $\mathrm{SnO}_{2}$ and OLA/OA mix shown for reference with the key stretching $(\nu)$ and bending $(\delta)$ vibrational modes annotated. The key argument outlined in the text is that the blue line can be thought of as a summation of the black and red lines. B, y-separated absorbance of films on $\mathrm{CaF}_{2}$ with various post deposition treatments.

Figure 6.19 A shows the ATR absorbance of a drop cast PbSnTe sample (blue line), i.e. without the restriction imposed by the onset of $\mathrm{CaF}_{2}$ absorbance below $1000 \mathrm{~cm}^{-1}$. Firstly, there is no absorption from the QD sample at energies above $3600 \mathrm{~cm}^{-1}(\approx 2780 \mathrm{~nm})$. For immediate reference, and measured on the same instrument, the absorbance of pure $\mathrm{SnO}_{2} \mathrm{NPs}$ is shown (black line), from which we can see the existence of the broad Sn-OH absorption $\left(\approx 3500 \mathrm{~cm}^{-1}\right)$, and the very significant $\mathrm{Sn}-\mathrm{O}$ stretch ( $<$ $5000 \mathrm{~cm}^{-1}$ ) [237, 243, 244, 247-252]. Rooted $\mathrm{Sn}-\mathrm{OH}$ in this context meaning that the $\mathrm{O}$ is provided by the $\mathrm{SnO}_{2}$ not the adsorbed water. The other features in the QD absorption in Figure 6.19 are explained by the oleic 
acid (OA) and oleylamine (OLA) ligands that are present. As the unique $\mathrm{Sn}-\mathrm{O}$ stretch is seen with the QDs, the existence of an amorphous $\mathrm{SnO}_{2}$ shell can be inferred. The ratio of the Sn-O stretch to the feature at 3200 $\mathrm{cm}^{-1}$ for the pure $\mathrm{SnO}_{2}$ is 34:1. As this ratio is observed for $100 \mathrm{~nm} \mathrm{NPs,}$ any lower ratio can only be caused by a higher surface to volume ratio of $\mathrm{SnO}_{2}$. For the PbSnTe QDs, for which the stretches are unobscured by the band edge and can thus be more accurately measured, this ratio is less than 2:1. Along with the confirmation provided by XPS of the Sn binding states at the surface, as TEM and XRD also prove that the samples are $\mathrm{SnTe}$ or PbSnTe crystals the only explanation is that there are $\mathrm{SnO}_{2}$ surface regions for both types of QDs.

Water molecules in atmosphere quickly adsorb to $\mathrm{SnO}_{2}$ surfaces via the mechanism shown in the first two images of Figure 6.20. The water homolytically disassociates as the lattice oxygen site reacts with a neutral $\mathrm{H}$ atom, forming the rooted $\mathrm{HO}_{\text {lat }}$ group, which is the origin of the stretch shown in Figure 6.19 as $\nu \mathrm{Sn}-\mathrm{OH}[246,248,249,252,253]$. This rooted group is then ionised, donating an electron to the conduction band. Additionally, the remaining $\mathrm{OH}$ group forms a weak dipole bond with the lattice $\mathrm{Sn}$. This associated hydroxyl group has a stretch seen close to the usual $\nu$ $\mathrm{O}-\mathrm{H}$ position $[246,253]$. The mechanism for this is shown in Figure 6.20. These two dissociated hydroxyl groups have active IR modes at exactly the observed wavenumber of the static feature at $3200 \mathrm{~cm}^{-1}[237,243-245]$. Together they account for the MIR absorbance feature in these QDs. It is key to note that for the PbSnTe QDs shown in Figure 6.19 A, the band edge is not in the MIR. However, for the SnTe QDs which do have an MIR band edge, the measured absorbance is given by a summation of features (Figure 6.2).

Figure 6.19 B shows the same film with three different capping ligands. The OA capped sample is shown for reference. The EDT capped film 


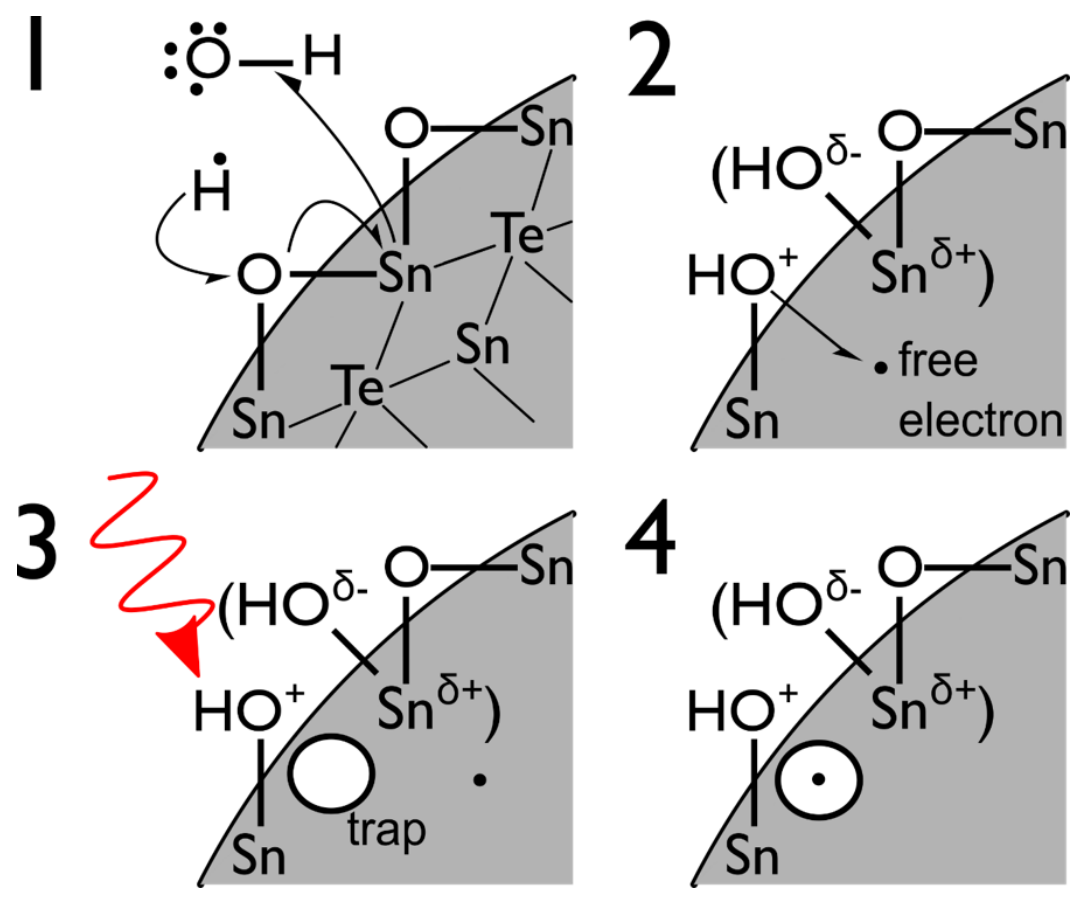

Figure 6.20: The steps of the water dissociation process at the $\mathrm{SnO}_{2}$ surface are: 1) $\mathrm{SnO}$ rich surface in the presence of homolytically disassociated atmospheric water. The lone $\mathrm{H}$ bonds with a rooted lattice oxygen, forming the rooted $\mathrm{HO}_{\text {lat }}$ group. The remaining associated $\mathrm{OH}$ group then forms a weak dipole with the lattice Sn $[248,249]$. 2) The rooted $\mathrm{OH}$ group is ionised and donates an electron to the conduction band in the surface region, which can contribute to hopping transport. 3) The molecular vibration of the rooted $\mathrm{OH}+$ resonates with incident MIR radiation. Vibronic coupling occurs between the vibrational state and the bulk electrical state [236]. This creates a new state that behaves as an electron trap. 4) An electron from the conduction band is trapped, reducing the carriers available for hopping, thus the bulk observable effect of incident MIR light is a reduction in measured current at constant voltage.

shows a clear relative reduction in the $\mathrm{OH}$ feature size. This implies that this strongly binding ligand has passivated the surface more thoroughly than both of the other ligands shown and displaced surface $\mathrm{OH}$. This can also be confirmed by the reduction in the $\delta \mathrm{O}-\mathrm{H}$ feature for the EDT treated film. Although useful spectroscopically, the EDT treated films did not show a conduction increase or any detectable photoresponse. The $\mathrm{As}_{2} \mathrm{~S}_{3}$ treated film, used in all the devices reported, shows an almost complete 
reduction in stretches related to alkyl ligands and leaves just the $\mathrm{Sn}-\mathrm{OH}$ (rooted) / O-H (associated) feature. Further, although arguably circumstantial, evidence of surface oxidation is provided by the stark variance in oxygen adsorption between Te and SnTe materials and is illustrated in Figure 6.21. Figure 6.21 shows that oxygen binds preferentially to Sn over Te and that the measured oxygen in TEM EDS is not an artefact of a noise signal from areas of high material density, relative to the TEM grid.

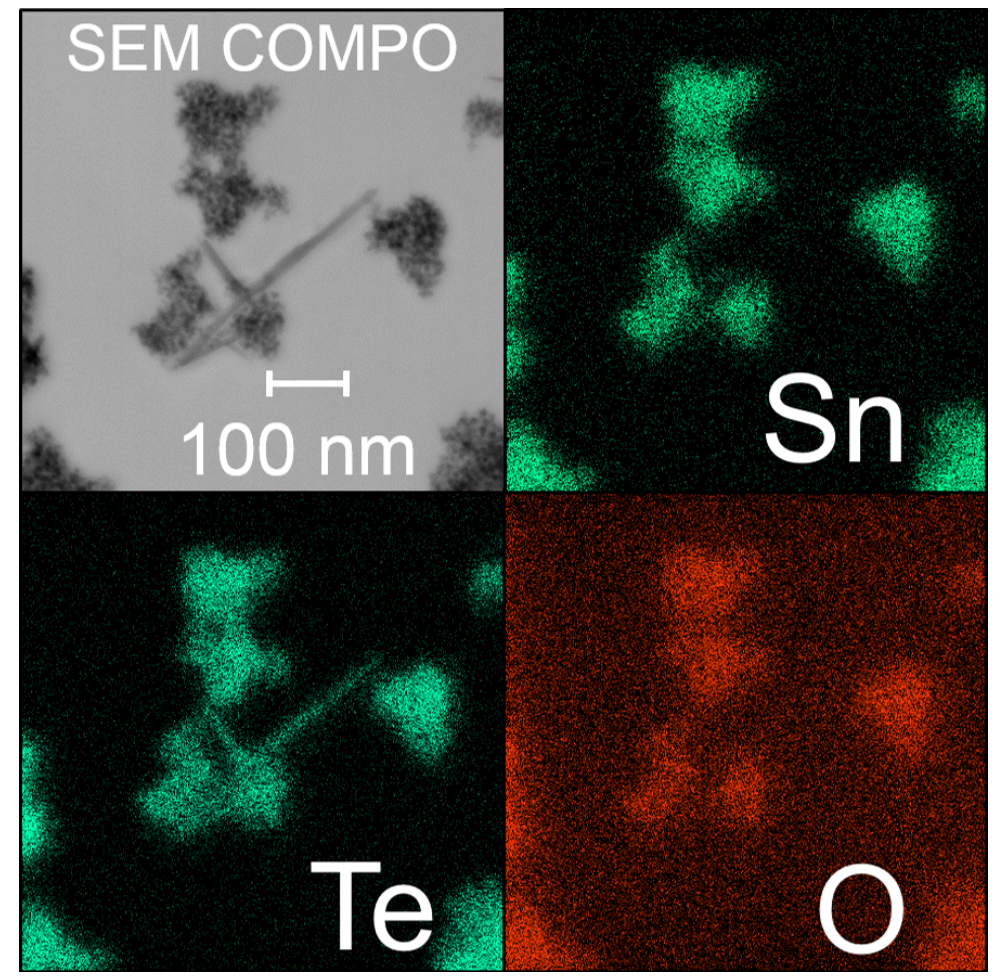

Figure 6.21: STEM EDS mapping of a SnTe prep with a sufficiently low OLA content to allow the formation of Te NWs (due to a lack of Sn coordination). It can be seen that the $N W$ features do not show any oxygen signal, whereas the QD clusters do show an oxygen signal above the background. It is clear that within the same colloidal suspension the Sn surfaces have adsorbed oxygen and the purely Te surfaces have not. This means that the $\mathrm{Sn}$ is the preferred oxygen bonding site over Te. There is no lead in this sample.

The same film changes that allow measurable photoresponse also come at the expense of QD surface passivation. It is well established that heating 
$\mathrm{SnO}_{2}$ will drive off some hydroxyl species, [237,245] however using high temperatures $\left(>300{ }^{\circ} \mathrm{C}\right)$ here would sinter the film. However, it has been found that the weakly bound associated hydroxyls can be temporarily replaced with OD (deuterium) groups if exposed to $\mathrm{D}_{2} \mathrm{O}$ at room temperature. This exchange was performed on a film that would be identical to the film on the devices reported above. The results are shown in Figure 6.22. The rooted $\mathrm{Sn}-\mathrm{OH}$ stretch is unaffected, but when there is a measurable

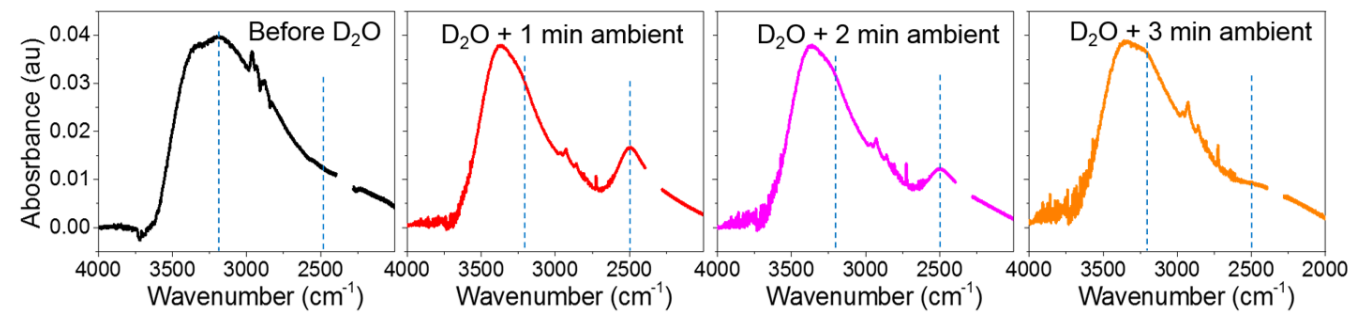

Figure 6.22: $A s_{2} S_{3}$ treated film of $Q D$ s, showing effect of $D_{2} \mathrm{O}$ exposure that replaces some OH groups, with $O D$ groups. The left hand line is at the same position in all 4 plots and shows the position of the associated hydroxyl O-H stretch, the right hand line is at the expected position for the $O-D$ stretch.

O-D stretch (right hand line) there is also a reduction in the absorbance region for the associated hydroxyl (left hand line). It has been reported that the rooted $\mathrm{OH}$ group remains on $\mathrm{SnO}_{2}$ surfaces even after outgassing at $773 \mathrm{~K}$ and major OD replacement has only been achieved after $48 \mathrm{~h}$ of $\mathrm{D}_{2} \mathrm{O}$ vapour at $633 \mathrm{~K}[245,254]$. Additionally with the geometry in a QD film, the only surface groups that would be available for this exchange are those associated groups that are not morphologically encapsulated in the film and hence, for both of these reasons, full exchange would not be expected. The total intensity of the absorbance loss at the $\mathrm{O}-\mathrm{H}$ position matches closely to the increased absorbance at the O-D position. After several minutes in ambient atmosphere the OD group disappears and the feature regains its original shape. The fact that a change of feature shape is observed implies that there is a group replacement, rather than just excess OD in the system. 


\subsubsection{Electrical Response}

The results above conclusively establish that the semiconductor QDs develop an oxide surface, that this surface interacts with atmospheric water and that this results in the absorption spectra observed. The electrical response to incident MIR must then, due to the fact that PbSnTe devices which have no MIR bandedge yet have a photoresponse, be due to an interaction between the adsorbed $\mathrm{OH}$ groups and incident radiation. This is not unlikely, as it is again well established that the adsorption process shown in Figure 6.20 causes an increase in electron density in the material $[248,249,252]$. The evidence suggests that when the vibrational stretch of the adsorbed species is excited by incident radiation there is a vibronic coupling [255] between the vibrational state of the Sn-OH stretch and the bulk electrical states that creates a new localised state [236, 256,257]. This state could trap a free electron, thus reducing the effective carrier density and decreasing conductivity of the film. This model is summarised in its entirety in Figure 6.20, and although not previously observed in tin chalcogenides specifically, explains the inverse photoresponse, the MIR absorption, the resonant electrical coupling and the behaviour of the device in wet air, dry nitrogen, and vacuum atmospheres. Clearly, further extensive work must be done to establish the veracity of this claim, however, with the current evidence this is the best explanation.

The presence of adsorbed species from hydroxyl groups on the (oxidised) surface explains the change in conduction by a change in the carrier density of the nanocrystal [247-249,252]. This type of surface doping has been well established for a variety of oxide surfaces commonly used for reversible gas sensing $[235,252,258-260]$. The mechanism of carrier doping involves a change in available carriers due to the specific species that is bound at the surface. This idea is illustrated by Figure $6.23 \mathrm{~A}$ and B which shows the absorbance of treated SnTe and PbSnTe QD films in air and in vacuum, respectively. In both films the absorbance is identical apart 

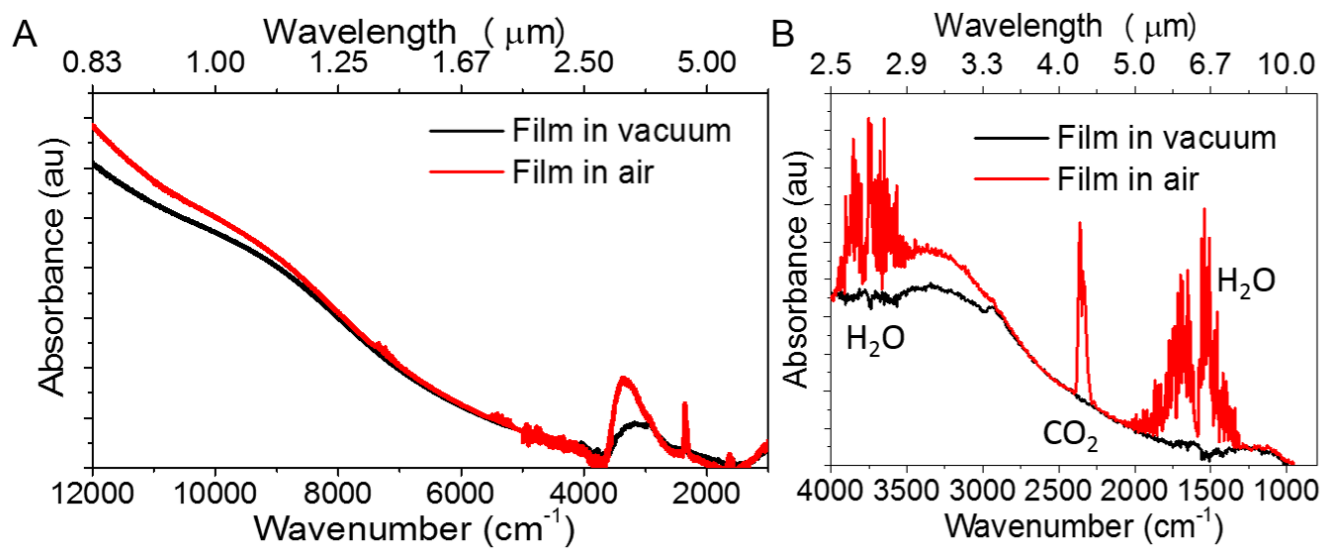

Figure 6.23: $A$, NIR-MIR absorbance of same PbSnTe film in air and vacuum. $B$, the MIR absorbance of the same SnTe film in air and in vacuum, for both $A$ and $B$, aside from the expected atmospheric $\mathrm{H}_{2} \mathrm{O}$ and $\mathrm{CO}_{2}$ changes in the path, the absorbance of the films fit except for in the region of interest $\left(3000-3500 \mathrm{~cm}^{-1}\right)$. The vacuum has clearly changed the absorbance of the film.

from the $\mathrm{OH}$ caused feature around $3200 \mathrm{~cm}^{-1}$. This evidence shows that a proportion of the absorbing species are reversibly removed by placing the film in a vacuum (or more slowly, in dry nitrogen), which indicates that surface species are playing a dominant role in the conductivity. This, coupled with the fact that the associated hydroxyl group can be replaced with an OD group strongly implies that surface groups are responsible for conduction and that these surface groups are water based. As there is no conventional photoconductive behaviour observed for SnTe, it is reasonable to conclude that photo-induced carriers in the SnTe core of the NC are not contributing strongly to conduction. The rooted hydroxyl group, which is known to increase the electron carrier density is responsible for providing excess carriers that give the high dark current. As shown in Figure 6.24 C and $\mathrm{D}$, applying a vacuum or nitrogen atmosphere $(1 \mathrm{~atm})$ quickly causes a severe but reversible change in conduction in the dark. Low conductivity in the presence of nitrogen is due to the "natural" conduction in the QD film, essentially hopping conduction that occurs without the increase in surface free carrier density caused by the rooted hydroxyl groups on the 
QD surface.

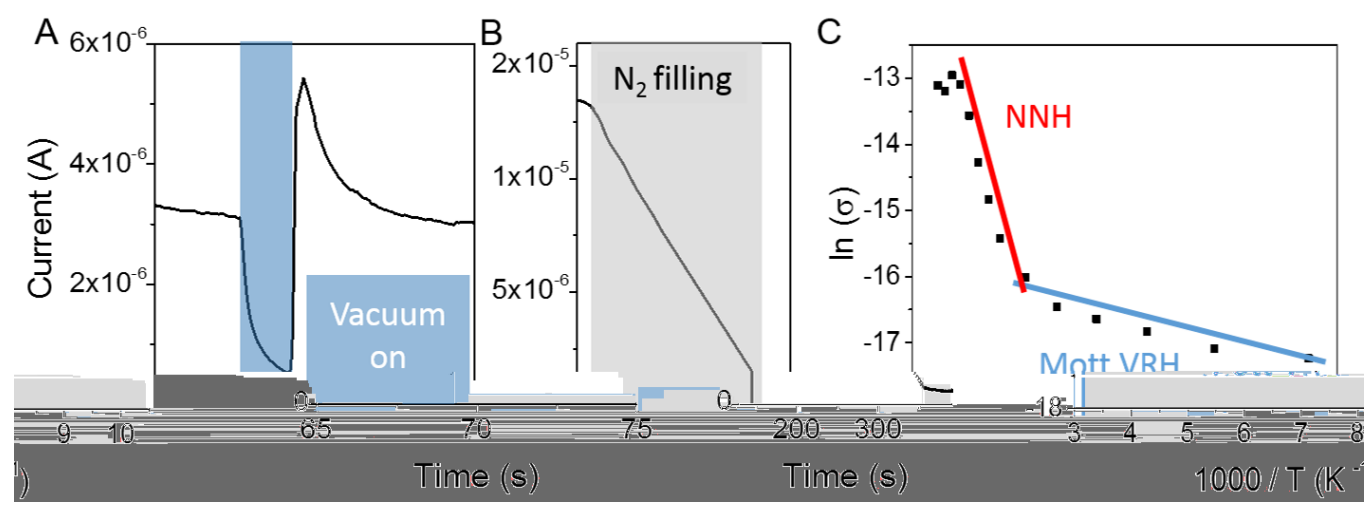

Figure 6.24: A, electrical behaviour of the device over time as a vacuum is applied, 10 Vbias. B, electrical behaviour of the device over time as $N_{2}$ is slowly let in to the chamber, this is achieved using a needle valve on the cryostat, 10 Vbias. C, conductivity dependence on temperature for the film under nitrogen (1 atm) where SnTe acts as a typical QD film with a Mott-VRH regime below $200 \mathrm{~K}$ and 1/T dependent NNH regime above $200 \mathrm{~K}$.

To emphasize this effect, the temperature dependence of the dark film conductivity in nitrogen is shown in Figure 6.24 C. Between $100 \mathrm{~K}$ and 200 $\mathrm{K}$ the conductivity proceeds via Mott variable range hopping (VRH) [48]. The localisation length for the Mott VRH is estimated at $5 \mathrm{~nm}$ which is small relative to the SnTe QD size in the film and therefore implies a low absolute conductivity in the system in this temperature range. At $200 \mathrm{~K}$, there is a clear crossover to an $\exp (1 / T)$ dependence that is indicative of nearest neighbour hopping (NNH), this is common in QD films and has previously been observed in, among other systems, PbSe, CdSe, CdTe and $\mathrm{ZnO}[40,197,261,262]$. Fitting this to an Arrhenius fit yields an activation energy of $268 \mathrm{meV}$, a value that is higher than that reported for similar QD systems [43] and so indicates that even at higher temperatures, without the hydroxyl surface states, conduction through the film is severely inhibited. This is qualitatively to be expected if the effective hopping length not only includes the inter-QD separation but also any oxide region on the surface. We can also use the NNH fit to calculate the expected change in current if 
the photoresponse were due to a heating effect. At room temperature, the observed temperature change during measurement would cause the current to increase by $<0.1 \mathrm{nA}$ (at $10 \mathrm{~V}$ ), not decrease by a much larger value, and thus cannot be the cause of the detection signal. This data also eliminates the chance that a pure $\mathrm{SnO}_{2}$ system is responsible for the observed behaviour since tin oxide at room temperature is known to have a higher conductivity under nitrogen than in air and displays a positive correlation of conductivity with temperature [263]. This is supported by the lack of photodetection from pure $\mathrm{SnO}_{2} \mathrm{NP}$ films in the same device geometry. All these observations show that the underlying QD scaffold is vital to the function of these devices and that the thin oxidised layer on the surface acts only as a hydroxyl bonding site that creates a measurable change in free carrier density in the conduction band of the SnTe core nanocrystals. It is interesting, from a technological standpoint, that these detectors do not respond to visible light. This is demonstrated in Figure 6.25, where Figure 6.25 A shows the operation of an HgTe device that is otherwise identical to the SnTe device. Similarly to previously described devices in Chapter 3 when the $670 \mathrm{~nm}$ laser diode (LD) is activated it causes a photocurrent in the HgTe device. Even the latest narrowband HgSe devices have interband absorptions at higher energies and thus require the use of an external longpass filter. In contrast the operation of the laser diode does not affect the behaviour of the SnTe device (Figure $6.25 \mathrm{~B}$ ) to incident IR light and thus an imaging device based on these SnTe detectors could not be blinded by visible wavelengths.

\subsection{Conclusion}

We have shown that the MIR absorbing states of surface species on SnTe and $\mathrm{PbSnTe}$ QDs can be used to detect MIR radiation at room temperature and with exposure to air during synthesis and fabrication. These simple planar devices display responsivities up to $6 \mathrm{~mA} \mathrm{~W}^{-1}$ at high bias and 

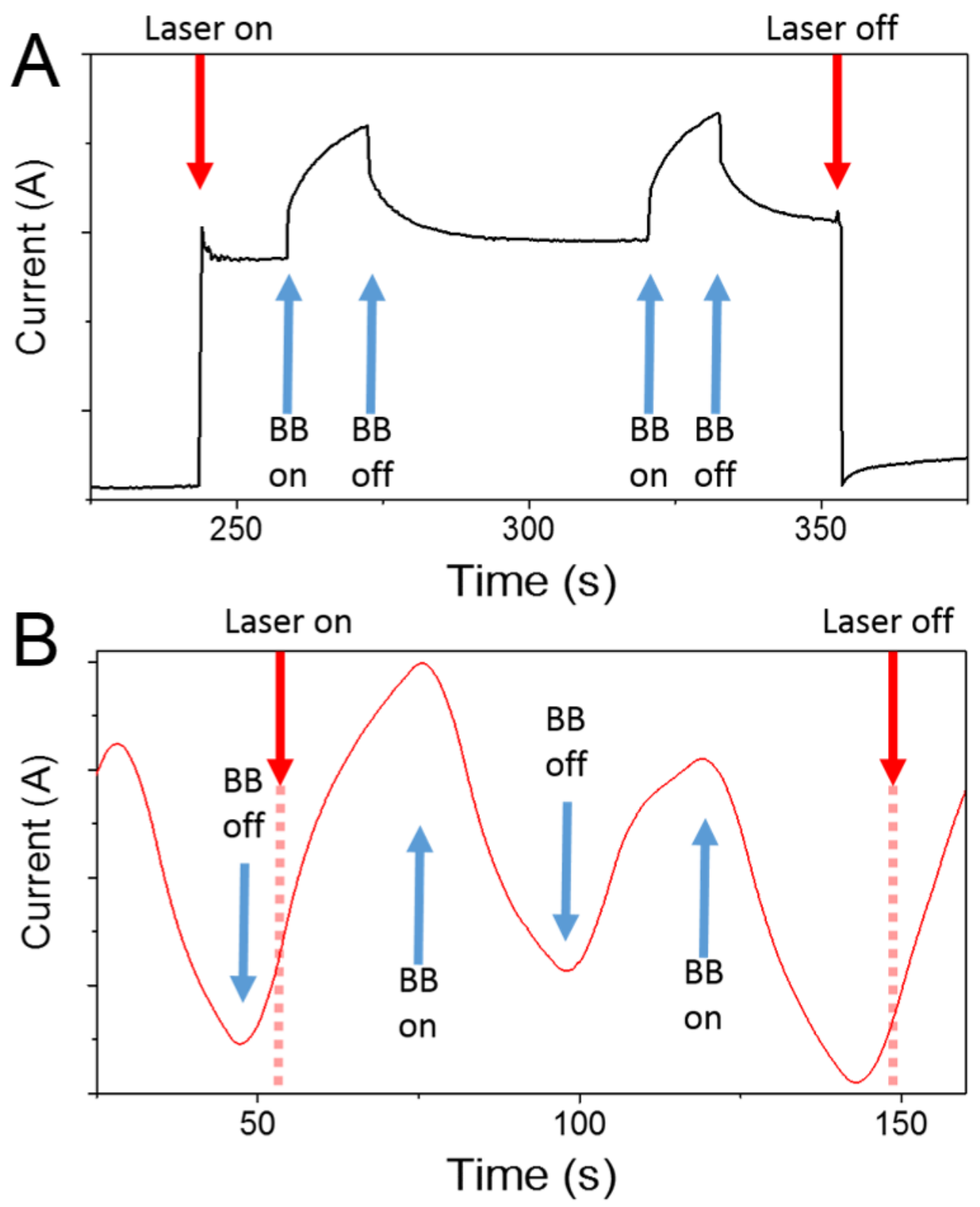

Figure 6.25: $A$, the operation of a conventional $\mathrm{HgTe} C Q D$ MIR detector at $1 \mathrm{~V}$ bias, the $670 \mathrm{~nm}$ laser diode (LD) causes a photocurrent that can blind the detector electronics at intensities above threshold. B, the SnTe device operated at $1 \mathrm{~V}$ bias. Although far slower than the HgTe device in this configuration, it displays an inverse photoresponse that is unaffected by the laser diode. 
$2 \mathrm{~mA} \mathrm{~W}^{-1}$ below $5 \mathrm{~V}$, to purely MIR radiation, and are resistant to exposure from higher energy light sources. From fitting the data to the known behaviour of $\mathrm{SnO}_{2}$ surfaces it appears that the molecular vibration of an adsorbed hydroxyl species is responsible for the detection of the MIR light. It is definitely the case that the behaviour presented here is a result of the surface chemistry on individual QDs within the film and thus represents one of the first applications of reversible surface doping in QD detector systems. The proposed mechanism of free carriers caused by adsorption of hydroxyl species to tin oxide sites is consistent with all observed data, is based on the well understood behaviours of tin surfaces and fits qualitatively within a hopping model. As such this represents the first verified observation of MIR detection using SnTe and PbSnTe QDs. With further refinement in the device performance, these materials could perhaps replace mercury NC based detectors in applications where toxicity is a major concern but the speed of imaging is not. Ultimately though it may be the light activated electrical sensing of a surface species, the mechanism for MIR sensing in SnTe, that makes this system uniquely interesting for further study. 


\section{Chapter 7}

\section{Conclusion and Further Work}

\subsection{Conclusion}

This work as a whole demonstrates that QDs can function as solution processable absorber layers in optoelectronic devices without any of the complexity of current semiconductor fabrication. Cheap and robust polymer materials offer superior functionality when used as the device substrate, which together with the solution based QD fabrication methods, offer a real opportunity to deliver optical sensors at a lower cost than those based on bulk semiconductors. As such there is definite commercial viability in pursuing planar QD devices as replacements for microbolometers. Alongside this, the discovery of 2-terminal charge memory over second timescales and the optical interactions at the surface of tin based QDs continue to demonstrate that QDs offer a large degree of yet to be discovered functionality when used in devices.

\subsubsection{HgTe Quantum Dots for MIR Imaging}

Colloidal HgTe QDs have continued to demonstrate that they are a very robust and stable material. As has been shown here, they offer a sharp easily controllable bandgap, an adaptable surface environment and a tol- 
erance to environmental impurities. With regard to their use in devices the key progress points proven in this work are:

- The colloidal bandgap is maintained with predictable redshift when forming solids, even with all ambient processing and fabrication. This means that the QDs are remaining spatially separated following the ligand exchange and the redshift is a result of wavefunction overlap or leakage. This is still an area of disagreement in the literature however it is more feasible in a $10 \mathrm{~nm} \mathrm{HgTe} \mathrm{QD} \mathrm{with} \mathrm{a} \mathrm{Bohr}$ radius of $42 \mathrm{~nm}$ than in a $5 \mathrm{~nm}$ CdSe QD with a Bohr Radius of 5.4 nm.

- Inorganic ligand exchange can deliver time response superior to thermal detectors in spite of oxygen surface trap sites. This is not to say that air exposed film performance is superior, or equal to inert processed devices. However as the time performance demonstrated here is superior to the in-use commercial competitor it acts as a sufficient proof of concept.

- Thermal dark current at room temperature is low enough that signal can be observed. This has not been achieved by minimising photoconductive dark current, but by showing that photogenerated carriers are sufficiently numerous that a signal can be detected at room temperature. This is due to a number of complementary processes. Firstly, the HgTe QDs must remain highly intrinsic even with surface effects. Secondly, the different thermal filling in each dot means that the room temperature dark current of the QD film is lower than a bulk crystal under the same condition. Thirdly, the lifetime of a photogenerated carrier must be longer than the hopping time, particularly with inorganic ligands, thus allowing the carriers to hop out of the dot under the effect of an applied field before recombination. This latter point may be due to a phonon bottleneck. 
- Organically capped HgTe QDs adhere more strongly as hydrophobicity of the surface increases. The use of these higher wetting angle materials eliminates the inorganic ligand exchange delamination problem found when using oxide materials as the substrate. This facilitates tuning of the ligand exchange such that the electrical response can be optimised, rather than being physically limited by film delamination.

- Spray coating from non-polar solvents can be used to create electrically active films that are spatially patterned, in only one fabrication step. As the size of these areas decreases the electrical performance of the film is predictable. This removes the possible film degradation that would occur from a further patterning step that is conducted on a large film.

- Device response with an EQE greater than 1 has been demonstrated in a number of devices and with multiple sizes of QD. This allows devices to detect a calibrated target source that is only $75{ }^{\circ} \mathrm{C}$ warmer than the device itself. This suggests that the idea of using MIR, rather than LWIR, signals for detection of objects in the ambient environment is now possible. Accordingly, if MIR radiation becomes detectable by affordable devices then there will no longer be a technical barrier to the production of multicolour MIR devices.

Together, the work presented here acts as proof of a number of the postulated benefits of QD use in optoelectronic devices. It has been shown that solution processability is of benefit in reducing complexity in fabrication and facilitating simple patterning. That bandgap tuning can offer enhanced device functionality through threshold temperature detection regimes and that photoconductive gain through charge trapping can increase device sensitivity. 


\subsubsection{Memory Applications}

Through an investigation of reproducible electronic hysteresis it has been shown that oxygen based hole traps cause charge memory in a 2-terminal device. The behaviour of these traps can be manipulated by temperature, illumination and 3rd terminal gating. These devices are not competitive with modern flash and RAM memory currently, but the large number of control parameters and very simple operation may lead to new designs of memory device in the future. If the trapping time of a hole is on the order of milliseconds, as suggested by the behaviour shown here, then it is not unfeasible that single QDs can demonstrate refresh rates and power use that is superior to current RAM modules. If that is the case, as a single QD occupies a much smaller volume than the CMOS based architecture currently used, it may be possible to use a single QD as a memory bit in the future.

\subsubsection{Tin based Quantum Dots for Sensing}

From a QD synthesis and process perspective SnTe and PbSnTe QDs behave very similarly to HgTe QDs. However, when placed in a planar device architecture the Sn-based QDs show completely different behaviour. Through a spectroscopic investigation it has been shown that the interaction with water at the Sn surface fundamentally changes the electronic behaviour of the QD films in a way not seen with HgTe QDs. Due to the hopping conduction between QDs the charge density in the surface region has a large effect on the measured electrical behaviour. The $\mathrm{O}-\mathrm{H}$ vibration of adsorbed water is in the MIR region and it was discovered that the resistance of the films increased when they were illuminated, but only by MIR radiation. The interaction between MIR radiation and the surface species reduces the charge density at the surface of the dot, which is the first report of an optoelectronic QD device that operates via an interaction between light and a surface species. The devices reported here are 
not suitable for imaging at the current time but with further investigation it may be possible to develop a device that can be optically sensitised by attaching different molecules at the surface.

\subsection{Further Work}

\subsubsection{HgTe QDs}

As is evident from the work shown in this thesis, this field is still very much in its infancy and there are still a wide variety of areas that need further research. Several obvious ones are:

- The electrical behaviour during absorption in inorganically capped films. Why do devices seem to have a wavelength dependent electrical response, beyond the simple band edge/photoconductive model. Transient Absorption Spectroscopy would be an ideal candidate to use on these films however MIR TAS is not yet commonly available. Is the postulate from chapter 5 that the observed responses are due to a numerical interaction of photogenerated carriers with trapped carriers correct?

- Trap state energetics; where are the oxygen derived trap states and how deep are they? How long lived is an individual trap? Is it long enough to potentially design a bespoke memory device?

- Do the generation, recombination and conduction mechanics allow true to life simulations of QD film systems? If not, what theoretical piece is missing? The creation of a simulated QD film that can show the behaviours reported here should be a priority for any group looking to combine experimental device prototyping with an understanding of novel behaviours.

- The production of an architecture that allows an array of $160 \times 120$ planar pixels needs to be developed. The aspiration should be 30 
$\mu \mathrm{m}$ square pixels comprised of $8,2 \mu \mathrm{m}$ trenches. The difficulty will be in contacting both rails of each pixel probably using a number of photoresist layers with pinholes.

- Film optimisation and passivation techniques, the opportunity for passivation that more thoroughly protects the surface but without affecting electrical behaviour is vast. As a starting point, a well controlled study investigating other inorganic ligands should be conducted. Using as metrics the MIR responsivity and dark current noise. Following from this an extensive process optimisation for the best performing ligand should be conducted. Specifically a large controlled study investigating ligand concentration in the exchange solution and film parameters such as thickness or solution concentration. Once this work has delivered an optimised film then a study on film passivation should be undertaken, there are a large range of potential options. Initially an investigation of the effect of halide acids and metal halide salts on the film should be conducted, both before and after ligand exchange. The intent being a passivation that delivers films that do not change their electrical behaviour over time, show the sharpest possible band edge and give the quickest rise time for MIR radiation.

\subsubsection{Ambipolar Transistor Behaviour}

As briefly remarked upon when discussing ambipolar transistor behaviour, the integration of observed effects in NC film transistors with physical theory is one area that could yield interesting results. Individual QDs should be well understood as surface effect dominated bulk semiconductors with an altered band structure, and theoretical work to integrate models of ensembles of individual QDs into a bulk conduction model could be easily confirmed or denied with relatively simple experiments. Perhaps this would then lead to enhanced device 
functionality as passivation methodology advances in line with accurate modelling. This could be attached to an investigation of the bias stress effect. The road map for such an investigation would begin with work to fabricate electrically similar transistors. Once a highly reproducible device was possible then the bias stress effect could be investigated under difficult temperature, optical and environmental conditions. This could also extend to a study of the dielectric surface itself, both oxide material and any surface functionalisation molecules present.

\subsubsection{Memory Behaviour}

If directly following on from the work described here then the first objective would be to create a highly reproducible device, in terms of quantitative behaviour rather than just qualitative. Without that any further optimisation would be impossible to correctly ascribe. Once achieved an investigation into the optimal amount of oxygen exposure should be conducted, which would likely also involve a process that optimises the organic ligand exchange for the minimum surface passivation that also gives an easily measurable on current. Following from that the objective would be to maximise trap lifetime per dot, which, as a first guess, is likely to be a feature of QD composition and size. Concurrently a project to shrink the device architecture to sub-micron feature size would be very beneficial, even if the only way to do so is via electron beam lithography as an initial proof of concept.

\subsubsection{SnTe Devices}

The number of potential follow on projects from this work is very large but some options are, to develop a QD synthesis method that gives a monodisperse sample as the soft band edge and inhomogeneity in the sample must be improved. As the ligand used is critical in determining the electrical behaviour and the absorption of the film, a more involved study using a large 
number of ligands would help to strictly clarify what is actually happening in the film. Finally although very difficult it is definitely worthwhile to try and synthesise the QDs in a deuterium rich environment followed by purification and optical characterisation in an inert environment. Purely deuterium capped QDs would be a fascinating tool with which to probe the proposed mechanism.

\subsection{Summary}

This work has detailed the steps taken to synthesise two families of QD, develop and fabricate two main device types on a wide number of substrate materials, design and build two optoelectronic test suites and details the characterisation of numerous devices. The main thrust of the work has delivered devices that show the potential of QDs from a fabrication perspective but also perform at or above the level in the published literature for similar device designs. Furthermore, a new interaction of MIR radiation with SnTe QD films was identified along with an investigation that clarified features that had been observed but not convincingly investigated in the prior literature. Finally, the electrical hysteresis in air exposed $\mathrm{HgTe}$ films was characterised leading to a report of electrical memory that originates at the surface of individual QDs. 


\section{Bibliography}

[1] Eric Mounier. Uncooled Infrared Imagers - Market E Technology report. Yole Developpement, Lyon, France, August 2017. 1, 2, 76

[2] Yann de Charentenay. Infrared Detectors - Technology and Market Trends. Yole Developpement, Lyon, France, October 2015. 1, 2, 76

[3] Manijeh Razeghi and Binh-Minh Nguyen. Advances in mid-infrared detection and imaging: a key issues review. Reports on Progress in Physics, 77(8):082401, 2014. 1, 27

[4] Antoni Rogalski. Infrared detectors. Taylor \& Francis, Boca Raton, 2nd edition, 2011. $1,8,28,67,68,69,77,239$

[5] E. Lhuillier, S. Keuleyan, H. Liu, and P. Guyot-Sionnest. Mid-IR Colloidal Nanocrystals. Chemistry of Materials, 25(8):1272-1282, April 2013. 1, 28, 40, 43, 45

[6] E. H. Sargent. Infrared Quantum Dots. Advanced Materials, 17(5):515-522, March 2005. 1,42

[7] Jason Palidwar Technologies, Iridian Spectral. Optical Filters Open Up New Uses for MWIR, LWIR Systems. 1

[8] Marc P. Hansen and Douglas S. Malchow. Overview of SWIR detectors, cameras, and applications. In Thermosense X, volume 6939, page 69390I. International Society for Optics and Photonics, March 2008. 1, 76

[9] Nibir K., Ravi Dat, and Ashok K. Advances in Infrared Detector Array Technology. In Sergei Pyshkin, editor, Optoelectronics - Advanced Materials and Devices. InTech, January 2013. 1, 12

[10] Thirimachos Bourlai. Mid-wave IR face recognition systems. SPIE Newsroom, February 2013. 2

[11] Ioannis Pavlidis, Norman L. Eberhardt, and James A. Levine. Human behaviour: Seeing through the face of deception. Nature, 415(6867):35, January 2002. 2, 76

[12] Jane Loveday, Grant Loveday, Josh Byrne, Boon-lay Ong, and Peter Newman. Quantifying radiation from thermal imaging of residential landscape elements. Renewable Energy and Environmental Sustainability, 2:17, 2017. 2, 76

[13] Infrared, January 2019. Page Version ID: 877289846. 3 
[14] John Lester Miller. Principles of infrared technology: a practical guide to the state of the art. Van Nostrand Reinhold, New York, 1994. 3, 6

[15] Morton Masius and Max Planck. The theory of heat radiation. P. Blakiston's Son \& Co., Philadelphia, 1914. 4

[16] P. W. Atkins, P. W. Atkins, and Julio De Paula. Atkins' Physical chemistry. Oxford University Press, Oxford ; New York, 7th edition, 2002. 5, 60, 62, 63

[17] William L Wolfe and George J Zissis. The infrared handbook. the infrared information analysis (iria) center. Environmental Research Institute of Michigan, 4th Printing, 1993. $6,7,9,28,32,65,67,68,73,239$

[18] Michael A. Kinch. Fundamental physics of infrared detector materials. Journal of Electronic Materials, 29(6):809-817. 7, 30, 168

[19] S. M. Sze. Semiconductor devices, physics and technology. Wiley, New York, 2nd edition, 2002. 7, 13, 16, 19, 21, 24, 140

[20] John David Vincent. Fundamentals of infrared and visible detector operation and testing. Wiley, Hoboken, New Jersey, 2nd edition, 2016. 8, 9, 12, 28, 30, 31

[21] Anthony J. Ciani, Richard E. Pimpinella, Christoph H. Grein, and Philippe GuyotSionnest. Colloidal quantum dots for low-cost MWIR imaging. volume SPIE Proceedings 9819, pages 981919-981919-9, 2016. 8, 27, 50, 163

[22] Safa O. Kasap. Optoelectronics \& Photonics: Principles \& Practices: International Edition. Pearson Higher Ed, 2013. 9, 10, 16, 19, 27, 30, 32, 33, 43

[23] Cherie R. Kagan, Efrat Lifshitz, Edward H. Sargent, and Dmitri V. Talapin. Building devices from colloidal quantum dots. Science, 353(6302):aac5523, August 2016. 12, $38,40,42,43,186$

[24] A. Hoffman. Semiconductor processing technology improves resolution of infrared arrays. Laser Focus World, 42(2):81-84, 2006. 12, 28

[25] Thomas Ihn. Semiconductor nanostructures: quantum states and electronic transport. Oxford University Press, Oxford ; New York, 2010. OCLC: ocn430496978. 12

[26] Jana Zaumseil and Henning Sirringhaus. Electron and Ambipolar Transport in Organic Field-Effect Transistors. Chemical Reviews, 107(4):1296-1323, April 2007. $12,13,14,15$

[27] Dmitri V. Talapin and Christopher B. Murray. PbSe Nanocrystal Solids for n- and pChannel Thin Film Field-Effect Transistors. Science, 310(5745):86-89, October 2005. 12

[28] J. C. Scott and L. D. Bozano. Nonvolatile Memory Elements Based on Organic Materials. Advanced Materials, 19(11):1452-1463, June 2007. 15, 16

[29] Ting-Chang Chang, Fu-Yen Jian, Shih-Cheng Chen, and Yu-Ting Tsai. Developments in nanocrystal memory. Materials Today, 14(12):608-615, December 2011. 15, 16

[30] International Technology Roadmap for Semiconductors 2.0. 15, 124 
[31] Bart V Van Zeghbroeck. Principles of semiconductor devices and heterojunctions. Prentice Hall, Upper Saddle River, N.J.; London, 2010. OCLC: 456836708. 16

[32] Jin Young Kim, Oleksandr Voznyy, David Zhitomirsky, and Edward H. Sargent. 25th Anniversary Article: Colloidal Quantum Dot Materials and Devices: A Quarter-Century of Advances. Advanced Materials, 25(36):4986-5010, September 2013. $18,36,42,45$

[33] Peter Y. Yu and Manuel Cardona. Fundamentals of semiconductors: physics and materials properties. Graduate texts in physics. Springer, Berlin, fourth edition, 2010. OCLC: 390878032.20

[34] E. Fred Schubert. Physical Foundations of Solid-State Devices. E. Fred Schubert, March 2015. Google-Books-ID: 15gSBwAAQBAJ. 20

[35] Jingshan Zhang and Boris I. Shklovskii. Density of states and conductivity of a granular metal or an array of quantum dots. Physical Review B, 70(11):115317, September 2004. 21

[36] Philippe Guyot-Sionnest. Electrical Transport in Colloidal Quantum Dot Films. The Journal of Physical Chemistry Letters, 3(9):1169-1175, May 2012. 21, 22, 23, 24, 25, 26, 43,103

[37] Gerasimos Konstantatos, editor. Colloidal quantum dot optoelectronics and photovoltaics. Cambridge University Press, New York, 2013. 21, 22

[38] Charles Kittel. Introduction to solid state physics. Wiley, Hoboken, NJ, 8th ed edition, 2005. 22,23

[39] RJ Brown, MJ Kelly, R Newbury, M Pepper, B Miller, H Ahmed, DG Hasko, DC Peacock, DA Ritchie, JEF Frost, and GAC Jones. The one dimensional quantised ballistic resistance in GaAs/AlGaAs heterojunctions with varying experimental conditions. Solid-State Electronics, 32(12):1179-1183, December 1989. 23

[40] Dong Yu, Congjun Wang, Brian L. Wehrenberg, and Philippe Guyot-Sionnest. Variable Range Hopping Conduction in Semiconductor Nanocrystal Solids. Physical Review Letters, 92(21):216802, 5 2004. 24, 149, 181, 218

[41] International Union of Pure and Applied Chemistry. IUPAC Gold Book - Arrhenius equation. 24

[42] Jiang Tang and Edward H. Sargent. Infrared Colloidal Quantum Dots for Photovoltaics: Fundamentals and Recent Progress. Advanced Materials, 23(1):12-29, January 2011. 24

[43] Sean Keuleyan, Emmanuel Lhuillier, Vuk Brajuskovic, and Philippe GuyotSionnest. Mid-infrared HgTe colloidal quantum dot photodetectors. Nature Photonics, 5(8):489-493, August 2011. 24, 47, 49, 73, 76, 93, 112, 120, 218

[44] Yao Liu, Markelle Gibbs, James Puthussery, Steven Gaik, Rachelle Ihly, Hugh W. Hillhouse, and Matt Law. Dependence of Carrier Mobility on Nanocrystal Size and Ligand Length in PbSe Nanocrystal Solids. Nano Letters, 10(5):1960-1969, May 2010. $24,40,47$ 
[45] Heng Liu, Alexandre Pourret, and Philippe Guyot-Sionnest. Mott and EfrosShklovskii Variable Range Hopping in CdSe Quantum Dots Films. ACS Nano, 4(9):5211-5216, September 2010. 25, 26

[46] Abdullah Yildiz, Necmi Serin, Tülay Serin, and Mehmet Kasap. Crossover from Nearest-Neighbor Hopping Conduction to Efros-Shklovskii Variable-Range Hopping Conduction in Hydrogenated Amorphous Silicon Films. Japanese Journal of Applied Physics, 48(11R):111203, November 2009. 25

[47] Dong Yu, Congjun Wang, Brian L. Wehrenberg, and Philippe Guyot-Sionnest. Variable Range Hopping Conduction in Semiconductor Nanocrystal Solids. Physical Review Letters, 92(21):216802, May 2004. 26

[48] N. F. Mott. Conduction in non-crystalline materials. Clarendon Press ; Oxford University Press, Oxford : New York, 1987. 26, 218

[49] B. I. Shklovskii and Aleksej L. Ėfros. Electronic properties of doped semiconductors. Number 45 in Springer series in solid-state sciences. Springer-Verlag Berlin Heidelberg $\mathrm{GmbH}$, Berlin Heidelberg, softcover reprint of the original 1st edition 1984 edition, 2013. 26

[50] Antoni Rogalski. Infrared detectors: an overview. Infrared Physics \& Technology, 43(3-5):187-210, June 2002. 27

[51] Robert Bogue. From bolometers to beetles: the development of thermal imaging sensors. Sensor Review, 27(4):278-281, September 2007. 27, 28

[52] A. Rogalski. HgCdTe infrared detector material: history, status and outlook. Reports on Progress in Physics, 68(10):2267, 2005. 27, 28, 29

[53] Antoni Rogalski. Progress in focal plane array technologies. Progress in Quantum Electronics, 36(2-3):342-473, March 2012. 27

[54] Philippe Guyot-Sionnest and John Andris Roberts. Background limited midinfrared photodetection with photovoltaic HgTe colloidal quantum dots. Applied Physics Letters, 107(25):253104, December 2015. 27, 44, 48, 49, 76, 89, 91, 120, 162

[55] Frank Niklaus, Christian Vieider, and Henrik Jakobsen. MEMS-based uncooled infrared bolometer arrays: a review. volume SPIE Proceedings 6836, pages 68360D68360D-15, 2007. 28

[56] Werner Martienssen. Semiconductors. In Springer Handbook of Condensed Matter and Materials Data, pages 575-694. Springer, 2005. 29, 34

[57] D. D. Coon and R. P. G. Karunasiri. New mode of IR detection using quantum wells. Applied Physics Letters, 45(6):649-651, September 1984. 29

[58] B. F. Levine. Quantum-well infrared photodetectors. Journal of Applied Physics, 74(8):R1-R81, October 1993. 29

[59] Liu. Schneider, Harald. Hui C. Semiconductor Quantum Wells and Intersubband. In Quantum Well Infrared Photodetectors, number 126 in Springer Series in OPTICAL SCIENCES, pages 13-44. Springer Berlin Heidelberg, 2006. DOI: 10.1007/978-3540-36324-8_3. 29 
[60] C. H. Grein, P. M. Young, and H. Ehrenreich. Minority carrier lifetimes in ideal InGaSb/InAs superlattices. Applied Physics Letters, 61(24):2905-2907, December 1992. 30

[61] D. L. Smith and C. Mailhiot. Proposal for strained type II superlattice infrared detectors. Journal of Applied Physics, 62(6):2545-2548, September 1987. 30

[62] Alexander Goushcha and Bernd Tabbert. Optical Detectors. In Frank Träger Prof, editor, Springer Handbook of Lasers and Optics, pages 503-562. Springer New York, 2007. DOI: $10.1007 / 978-0-387-30420-5$-9. 30, 31, 32

[63] Sheng S. Li. Proceedings of the Sixth International Symposium on Long Wavelength Infrared Detectors and Arrays: Physics and Applications. The Electrochemical Society, 1999. 30, 31

[64] Jamie Phillips. Evaluation of the fundamental properties of quantum dot infrared detectors. Journal of Applied Physics, 91(7):4590-4594, April 2002. 32, 42, 43

[65] J. B. Johnson. Thermal Agitation of Electricity in Conductors. Physical Review, 32(1):97-109, July 1928. 33

[66] Heng Liu, Emmanuel Lhuillier, and Philippe Guyot-Sionnest. 1/f noise in semiconductor and metal nanocrystal solids. Journal of Applied Physics, 115(15):154309, April 2014. 33, 112

[67] A. P. Alivisatos. Semiconductor clusters, nanocrystals, and quantum dots. Science, 271(5251):933, February 1996. 33, 34, 43

[68] Armando Rastelli, Suwit Kiravittaya, and Oliver G. Schmidt. Growth and control of optically active quantum dots. In Peter Michler, editor, Single Semiconductor Quantum Dots, NanoScience and Technology, pages 31-69. Springer Berlin Heidelberg, 2009. DOI: 10.1007/978-3-540-87446-1_2. 33

[69] E. H. Kennard. Zur Quantenmechanik einfacher Bewegungstypen. Zeitschrift für Physik, 44(4-5):326-352, 1927. 34

[70] R. Rossetti, S. Nakahara, and L. E. Brus. Quantum size effects in the redox potentials, resonance Raman spectra, and electronic spectra of CdS crystallites in aqueous solution. The Journal of Chemical Physics, 79(2):1086-1088, July 1983. 34

[71] R. C. Miller, D. A. Kleinman, W. T. Tsang, and A. C. Gossard. Observation of the excited level of excitons in GaAs quantum wells. Physical Review B, 24(2):1134-1136, July 1981. 34

[72] Christopher M. Evans, Laura C. Cass, Kathryn E. Knowles, Daniel B. Tice, Robert P. H. Chang, and Emily A. Weiss. Review of the synthesis and properties of colloidal quantum dots: the evolving role of coordinating surface ligands. Journal of Coordination Chemistry, 65(13):2391-2414, July 2012. 34, 37

[73] S. V. Gaponenko. Introduction to nanophotonics. Cambridge University Press, Cambridge ; New York, 2010. OCLC: ocn460059731. 34 
[74] Rolf Koole, Esther Groeneveld, Daniel Vanmaekelbergh, Andries Meijerink, and Celso de Mello Donegá. Size Effects on Semiconductor Nanoparticles. In Celso de Mello Donegá, editor, Nanoparticles, pages 13-51. Springer Berlin Heidelberg, 2014. DOI: $10.1007 / 978-3-662-44823-6 \_2.34,35$

[75] Louis Brus. Electronic wave functions in semiconductor clusters: experiment and theory. The Journal of Physical Chemistry, 90(12):2555-2560, June 1986. 35, 186

[76] Weiming Que. Excitons in quantum dots with parabolic confinement. Physical Review B, 45(19):11036-11041, May 1992. 35

[77] Stephen V. Kershaw, Andrei S. Susha, and Andrey L. Rogach. Narrow bandgap colloidal metal chalcogenide quantum dots: synthetic methods, heterostructures, assemblies, electronic and infrared optical properties. Chemical Society Reviews, 42(7):3033, 2013. 36, 42

[78] Michael A. Boles, Daishun Ling, Taeghwan Hyeon, and Dmitri V. Talapin. The surface science of nanocrystals. Nature Materials, 15(2):141-153, February 2016. 37, 38,186

[79] R. Rossetti, J. L. Ellison, J. M. Gibson, and L. E. Brus. Size effects in the excited electronic states of small colloidal CdS crystallites. The Journal of Chemical Physics, 80(9):4464-4469, May 1984. 37

[80] Mauricio Meyer, Christer Wallberg, Kazue Kurihara, and Janos H. Fendler. Photosensitized charge separation and hydrogen production in reversed micelle entrapped platinized colloidal cadmium sulphide. Journal of the Chemical Society, Chemical Communications, 0(2):90-91, January 1984. 37

[81] A. P. Alivisatos, A. L. Harris, N. J. Levinos, M. L. Steigerwald, and L. E. Brus. Electronic states of semiconductor clusters: Homogeneous and inhomogeneous broadening of the optical spectrum. The Journal of Chemical Physics, 89(7):4001-4011, October 1988. 37

[82] A. Henglein. Photochemistry of colloidal cadmium sulfide. 2. Effects of adsorbed methyl viologen and of colloidal platinum. The Journal of Physical Chemistry, 86(13):2291-2293, June 1982. 37

[83] M. O'Neil, J. Marohn, and G. McLendon. Dynamics of electron-hole pair recombination in semiconductor clusters. The Journal of Physical Chemistry, 94(10):43564363, May 1990. 37

[84] C. B. Murray, D. J. Norris, and M. G. Bawendi. Synthesis and characterization of nearly monodisperse $\mathrm{CdE}(\mathrm{E}=$ sulfur, selenium, tellurium) semiconductor nanocrystallites. Journal of the American Chemical Society, 115(19):8706-8715, September 1993. 38, 53

[85] C. B. Murray, S. Sun, W. Gaschler, H. Doyle, T. A. Betley, and C. R. Kagan. Colloidal synthesis of nanocrystals and nanocrystal superlattices. IBM Journal of Research and Development, 45(1):47-56, January 2001. 38

[86] Victor K. LaMer and Robert H. Dinegar. Theory, Production and Mechanism of Formation of Monodispersed Hydrosols. Journal of the American Chemical Society, 72(11):4847-4854, November 1950. 38 
[87] Dmitri V. Talapin, Andrey L. Rogach, Andreas Kornowski, Markus Haase, and Horst Weller. Highly Luminescent Monodisperse CdSe and CdSe/ZnS Nanocrystals Synthesized in a Hexadecylamine-Trioctylphosphine Oxide-Trioctylphospine Mixture. Nano Letters, 1(4):207-211, April 2001. 38

[88] Yadong Yin and A. Paul Alivisatos. Colloidal nanocrystal synthesis and the organic-inorganic interface. Nature, 437(7059):664-670, September 2005. 38

[89] Intermolecular and Surface Forces: Revised Third Edition. Elsevier Science \& Technology Books. 39

[90] Mark D. Peterson, Laura C. Cass, Rachel D. Harris, Kedy Edme, Kimberly Sung, and Emily A. Weiss. The Role of Ligands in Determining the Exciton Relaxation Dynamics in Semiconductor Quantum Dots. Annual Review of Physical Chemistry, 65(1):317-339, 2014. 40

[91] M. V. Kurik. Urbach rule. physica status solidi (a), 8(1):9-45, 1971. 40

[92] Maksym V. Kovalenko, Marcus Scheele, and Dmitri V. Talapin. Colloidal Nanocrystals with Molecular Metal Chalcogenide Surface Ligands. Science, 324(5933):1417-1420, June 2009. 40, 46, 103

[93] Angshuman Nag, Hao Zhang, Eric Janke, and Dmitri V. Talapin. Inorganic Surface Ligands for Colloidal Nanomaterials. Zeitschrift für Physikalische Chemie, 229(12):85-107, 2014. 40

[94] Sean E. Keuleyan, Philippe Guyot-Sionnest, Christophe Delerue, and Guy Allan. Mercury Telluride Colloidal Quantum Dots: Electronic Structure, Size-Dependent Spectra, and Photocurrent Detection up to $12 \mu \mathrm{m}$. ACS Nano, 8(8):8676-8682, August 2014. 40, 41, 49, 76, 120

[95] Sungwoo Kim, Taehoon Kim, Sang Hyuk Im, Sang Il Seok, Kang Wook Kim, Sungjee Kim, and Sang-Wook Kim. Bandgap engineered monodisperse and stable mercury telluride quantum dots and their application for near-infrared photodetection. Journal of Materials Chemistry, 21(39):15232-15236, September 2011. 40

[96] Guy Allan and Christophe Delerue. Tight-binding calculations of the optical properties of HgTe nanocrystals. Physical Review B, 86(16):165437, October 2012. 41, 186

[97] Mark Green, Gareth Wakefield, and Peter J. Dobson. A simple metalorganic route to organically passivated mercury telluride nanocrystals. Journal of Materials Chemistry, 13(5):1076-1078, April 2003. 41, 42, 76

[98] Sean Keuleyan, Emmanuel Lhuillier, and Philippe Guyot-Sionnest. Synthesis of Colloidal HgTe Quantum Dots for Narrow Mid-IR Emission and Detection. Journal of the American Chemical Society, 133(41):16422-16424, October 2011. 41, 47, 76

[99] Emmanuel Lhuillier, Sean Keuleyan, Pavlo Zolotavin, and Philippe GuyotSionnest. Mid-Infrared HgTe/As2s3 Field Effect Transistors and Photodetectors. Advanced Materials, 25(1):137-141, January 2013. 41, 48, 49, 76, 91, 93, 95, 112, 115, $120,140,142,162$ 
[100] Emmanuel Lhuillier, Sean Keuleyan, and Philippe Guyot-Sionnest. Optical properties of HgTe colloidal quantum dots. Nanotechnology, 23(17):175705, 2012. 41, 104, 129,131

[101] D. Leonard, M. Krishnamurthy, C. M. Reaves, S. P. Denbaars, and P. M. Petroff. Direct formation of quantum-sized dots from uniform coherent islands of InGaAs on GaAs surfaces. Applied Physics Letters, 63(23):3203-3205, December 1993. 42

[102] Andrey Rogach, Stephen V. Kershaw, Mike Burt, Mike T. Harrison, Andreas Kornowski, Alexander Eychmüller, and Horst Weller. Colloidally Prepared HgTe Nanocrystals with Strong Room-Temperature Infrared Luminescence. Advanced Materials, 11(7):552-555, May 1999. 42

[103] Maksym V. Kovalenko, Erich Kaufmann, Dietmar Pachinger, Jürgen Roither, Martin Huber, Julian Stangl, Günter Hesser, Friedrich Schäffler, and Wolfgang Heiss. Colloidal HgTe Nanocrystals with Widely Tunable Narrow Band Gap Energies: From Telecommunications to Molecular Vibrations. Journal of the American Chemical Society, 128(11):3516-3517, March 2006. 42

[104] Jason P. Clifford, Keith W. Johnston, Larissa Levina, and Edward H. Sargent. Schottky barriers to colloidal quantum dot films. Applied Physics Letters, 91(25):253117, December 2007. 42

[105] U. Bockelmann and G. Bastard. Phonon scattering and energy relaxation in two, one-, and zero-dimensional electron gases. Physical Review B, 42(14):8947-8951, November 1990. 44

[106] Tarek A. Ameen, Yasser M. El-Batawy, and A. A. Abouelsaood. Modeling of the quantum dot filling and the dark current of quantum dot infrared photodetectors. Journal of Applied Physics, 115(6):063703, February 2014. 44

[107] Gerasimos Konstantatos, Ian Howard, Armin Fischer, Sjoerd Hoogland, Jason Clifford, Ethan Klem, Larissa Levina, and Edward H. Sargent. Ultrasensitive solutioncast quantum dot photodetectors. Nature, 442(7099):180, July 2006. 46, 84, 162, 177

[108] M. Böberl, M. V. Kovalenko, S. Gamerith, E. J. W. List, and W. Heiss. Inkjet-Printed Nanocrystal Photodetectors Operating up to $3 \mu \mathrm{m}$ Wavelengths. Advanced Materials, 19(21):3574-3578, November 2007. 46, 77, 163, 179

[109] Nengjie Huo, Shuchi Gupta, and Gerasimos Konstantatos. $\mathrm{MoS}_{2}-\mathrm{HgTe}$ Quantum Dot Hybrid Photodetectors beyond 2 micron. Advanced Materials, page 160576, February 2017. 48, 49, 76, 115

[110] Mengyu Chen, Haipeng Lu, Nema M. Abdelazim, Ye Zhu, Zhen Wang, Wei Ren, Stephen V. Kershaw, Andrey L. Rogach, and Ni Zhao. Mercury Telluride Quantum Dot Based Phototransistor Enabling High-Sensitivity Room-Temperature Photodetection at $2000 \mathrm{~nm}$. ACS Nano, 11(6):5614-5622, June 2017. 49, 76, 77, 84, 112, 115, 120

[111] Xin Tang, Xiaobing Tang, and King Wai Chiu Lai. Scalable Fabrication of Infrared Detectors with Multispectral Photoresponse Based on Patterned Colloidal Quantum Dot Films. ACS Photonics, 3(12):2396-2404, December 2016. 49, 77, 103, 112, 120,163 
[112] Zhiyou Deng, Kwang Seob Jeong, and Philippe Guyot-Sionnest. Colloidal Quantum Dots Intraband Photodetectors. ACS Nano, 8(11):11707-11714, November 2014. 50, 209, 210

[113] Emmanuel Lhuillier, Marion Scarafagio, Patrick Hease, Brice Nadal, Hervé Aubin, Xiang Zhen $\mathrm{Xu}$, Nicolas Lequeux, Gilles Patriarche, Sandrine Ithurria, and Benoit Dubertret. Infrared Photodetection Based on Colloidal Quantum-Dot Films with High Mobility and Optical Absorption up to THz. Nano Letters, 16(2):1282-1286, February 2016. 50

[114] Xin Tang, Guang fu Wu, and King Wai Chiu Lai. Plasmon resonance enhanced colloidal HgSe quantum dot filterless narrowband photodetectors for mid-wave infrared. Journal of Materials Chemistry C, 5(2):362-369, 2017. 50

[115] Aicha Elshabini and Fred D. Barlow. Thin film technology handbook. Electronic packaging and interconnection series. McGraw-Hill, New York, 1998. 55, 57

[116] Thermo Fisher Scientific. FTIR Basics. 58

[117] R. F. Egerton. Physical principles of electron microscopy: an introduction to TEM, SEM, and AEM. Springer, New York, NY, 2005. OCLC: ocm61714990. 59, 60, 61

[118] Eustace L. Dereniak and G. D. Boreman. Infrared detectors and systems. Wiley series in pure and applied optics. Wiley, New York, 1996. 67, 239

[119] https://www.thorlabs.com/newgrouppage9.cfm?objectgroup_id= 6479. Thorlabs photo conductive sensors. 71

[120] If the reader is looking for a resource to enable them to get a feel for these calculations then the author recommends http://www.spectralcalc. com/blackbody_calculator/blackbody.php. the definitive references as used in this work are $[4,17,118]$, however if the reader is looking for slightly more approachable references the author recommends http://www.oceanopticsbook.info/view/light_and_radiometry/ level_2/blackbody_radiation and http://www.spectralcalc.com/ blackbody/blackbody.html. 74

[121] Yomna Abdelrahman, Pascal Knierim, Pawel W. Wozniak, Niels Henze, and Albrecht Schmidt. See Through the Fire: Evaluating the Augmentation of Visual Perception of Firefighters Using Depth and Thermal Cameras. In Proceedings of the 2017 ACM International Joint Conference on Pervasive and Ubiquitous Computing and Proceedings of the 2017 ACM International Symposium on Wearable Computers, UbiComp '17, pages 693-696, New York, NY, USA, 2017. ACM. 76

[122] Chandler Downs and Thomas E. Vandervelde. Progress in Infrared Photodetectors Since 2000. Sensors, 13(4):5054-5098, April 2013. 76

[123] A. Rogalski. Recent progress in infrared detector technologies. Infrared Physics $\mathcal{E}$ Technology, 54(3):136-154, May 2011. 76

[124] A. Rogalski and P. Martyniuk. InAs/GaInSb superlattices as a promising material system for third generation infrared detectors. Infrared Physics \& Technology, 48(1):39-52, April 2006. 76 
[125] Matthew E. Cryer, Holger Fiedler, and Jonathan E. Halpert. Photo-Electrosensitive Memristor Using Oxygen Doping in HgTe Nanocrystal Films. ACS Applied Materials $\mathcal{E}$ Interfaces, 10(22):18927-18934, June 2018. 76, 179

[126] Yuval Yifat, Matthew Ackerman, and Philippe Guyot-Sionnest. Mid-IR colloidal quantum dot detectors enhanced by optical nano-antennas. Applied Physics Letters, 110(4):041106, January 2017. 76

[127] E. Lhuillier, S. Keuleyan, H. Liu, and P. Guyot-Sionnest. Mid-IR Colloidal Nanocrystals. Chemistry of Materials, 25(8):1272-1282, April 2013. 76

[128] Stephen V. Kershaw, Mike Burt, Mike Harrison, Andrey Rogach, Horst Weller, and Alex Eychmüller. Colloidal CdTe/HgTe quantum dots with high photoluminescence quantum efficiency at room temperature. Applied Physics Letters, 75(12):16941696, September 1999. 76

[129] Jie Bao and Moungi G. Bawendi. A colloidal quantum dot spectrometer. Nature, 523(7558):67, July 2015. 76

[130] Emmanuel Lhuillier, Adrien Robin, Sandrine Ithurria, Herve Aubin, and Benoit Dubertret. Electrolyte-Gated Colloidal Nanoplatelets-Based Phototransistor and Its Use for Bicolor Detection. Nano Letters, 14(5):2715-2719, May 2014. 76

[131] Paul M. Hubel, John Liu, and Rudolph J. Guttosch. Spatial frequency response of color image sensors: Bayer color filters and Foveon X3. In Sensors and Camera Systems for Scientific, Industrial, and Digital Photography Applications V, volume 5301, pages 402-408. International Society for Optics and Photonics, June 2004. 77

[132] G. Konstantatos, C. Huang, L. Levina, Z. Lu, and E. H. Sargent. Efficient Infrared Electroluminescent Devices Using Solution-Processed Colloidal Quantum Dots. Advanced Functional Materials, 15(11):1865-1869, November 2005. 77

[133] Murat Tepegoz, Alper Kucukkomurler, Firat Tankut, Selim Eminoglu, and Tayfun Akin. A miniature low-cost LWIR camera with a $160 \times 120$ microbolometer FPA. In Infrared Technology and Applications XL, volume 9070, page 90701O. International Society for Optics and Photonics, June 2014. 77, 115

[134] P.V. Datskos and N.V. Lavrik. Detectors-Figures of Merit. In Encyclopedia of optical engineering, volume 1, pages 349-357. Marcel Dekker, New York, 2003. 77

[135] Han Yue Zheng, Omar A. Alsager, Bicheng Zhu, Jadranka Travas-Sejdic, Justin M. Hodgkiss, and Natalie O. V. Plank. Electrostatic gating in carbon nanotube aptasensors. Nanoscale, 8(28):13659-13668, 2016. 99

[136] Ahmad Rezaei Kolahchi, Abdellah Ajji, and Pierre. J. Carreau. Improvement of PET surface hydrophilicity and roughness through blending. AIP Conference Proceedings, 1664(1):030001, May 2015. 100, 108

[137] Lin-Wang Wang and Alex Zunger. Pseudopotential calculations of nanoscale CdSe quantum dots. Physical Review B, 53(15):9579-9582, April 1996. 102

[138] M G Bawendi, M L Steigerwald, and L E Brus. The Quantum Mechanics of Larger Semiconductor Clusters ("Quantum Dots"). Annual Review of Physical Chemistry, 41(1):477-496, 1990. 102 
[139] Y. Feng, S. Lin, S. Huang, S. Shrestha, and G. Conibeer. Can Tauc plot extrapolation be used for direct-band-gap semiconductor nanocrystals? Journal of Applied Physics, 117(12):125701, March 2015. 103

[140] Haitao Zhang, Bo Hu, Liangfeng Sun, Robert Hovden, Frank W. Wise, David A. Muller, and Richard D. Robinson. Surfactant Ligand Removal and Rational Fabrication of Inorganically Connected Quantum Dots. Nano Letters, 11(12):5356-5361, December 2011. 103

[141] X. W. Zhang and J. B. Xia. Electronic structure and electron $\mathrm{g}$ factors of HgTe quantum dots. Journal of Physics D: Applied Physics, 39(9):1815, 2006. 104

[142] Mark L. Brongersma, Naomi J. Halas, and Peter Nordlander. Plasmon-induced hot carrier science and technology. Nature Nanotechnology, 10(1):25-34, January 2015. 112

[143] William L Wolfe and George J Zissis. The Infrared Handbook. The Infrared Information Analysis (IRIA) Center. Environmental Research Institute of Michigan, 4th Printing, 1993. 112

[144] Selim Eminoglu. A 1024 by 768 digital roic for uncooled microbolometer fpas. In Infrared Technology and Applications XLIII, volume 10177, page 1017722. International Society for Optics and Photonics, June 2017. 115

[145] M. D. Fischbein and M. Drndic. CdSe nanocrystal quantum-dot memory. Applied Physics Letters, 86(19):193106, 5 2005. 124, 125

[146] Lei Wang, CiHui Yang, Jing Wen, and Shan Gai. Emerging Nonvolatile Memories to Go Beyond Scaling Limits of Conventional CMOS Nanodevices. Journal of Nanomaterials, page 927696. DOI: 10.1155/2014/927696. 124

[147] P. Mazumder, S. M. Kang, and R. Waser. Memristors: Devices, Models, and Applications. Scanning the Issue. Proceedings of the IEEE, 100(6):1911-1919. 124

[148] J. Joshua Yang, Dmitri B. Strukov, and Duncan R. Stewart. Memristive devices for computing. Nature Nanotechnology, 8(1):13-24. 124

[149] Yuriy V. Pershin and Massimiliano Di Ventra. Memory effects in complex materials and nanoscale systems. Advances in Physics, 60(2):145-227, 4 2011. 124, 125

[150] Ioannis Vourkas and Georgios Ch Sirakoulis. Memristor Fundamentals. Emergence, Complexity and Computation. Springer International Publishing. DOI: 10.1007/978-3-319-22647-7. 124, 135, 136

[151] Sascha Vongehr and Xiangkang Meng. The Missing Memristor has Not been Found. Scientific Reports, 5:11657, 6 2015. 124

[152] L. Chua. Memristor-The missing circuit element. IEEE Transactions on Circuit Theory, 18(5):507-519. 124

[153] L. O. Chua and Sung Mo Kang. Memristive devices and systems. Proceedings of the IEEE, 64(2):209-223. 124, 135

[154] Leon Chua. Resistance switching memories are memristors. Applied Physics A, 102(4):765-783, 3 2011. 124 
[155] Massimiliano Di Ventra and Yuriy V. Pershin. On the physical properties of memristive, memcapacitive and meminductive systems. Nanotechnology, 24(25):255201. 124

[156] YingTao Li, ShiBing Long, Qi Liu, HangBing Lü, Su Liu, and Ming Liu. An overview of resistive random access memory devices. Chinese Science Bulletin, 56(28-29):3072, 10 2011. 124, 125

[157] Dmitri V. Talapin, Jong-Soo Lee, Maksym V. Kovalenko, and Elena V. Shevchenko. Prospects of Colloidal Nanocrystals for Electronic and Optoelectronic Applications. Chemical Reviews, 110(1):389-458, 1 2010. 125

[158] Cherie R. Kagan, Efrat Lifshitz, Edward H. Sargent, and Dmitri V. Talapin. Building devices from colloidal quantum dots. Science, 353(6302):aac5523, 8 2016. PMID: 27563099. 125

[159] Michael A. Boles, Daishun Ling, Taeghwan Hyeon, and Dmitri V. Talapin. The surface science of nanocrystals. Nature Materials, 15(2):141-153. 125

[160] Y. Yang, J. Ouyang, L. Ma, R. J.-H. Tseng, and C.-W. Chu. Electrical Switching and Bistability in Organic/Polymeric Thin Films and Memory Devices. Advanced Functional Materials, 16(8):1001-1014, 5 2006. 125

[161] Byungjin Cho, Sunghun Song, Yongsung Ji, Tae-Wook Kim, and Takhee Lee. Organic Resistive Memory Devices: Performance Enhancement, Integration, and Advanced Architectures. Advanced Functional Materials, 21(15):2806-2829, 8 2011. 125

[162] Electric-field-induced charge transfer between gold nanoparticle and capping 2naphthalenethiol and organic memory cells. Applied Physics Letters, 86(12):123507, 3 2005. 125

[163] Jang-Sik Lee, Jinhan Cho, Chiyoung Lee, Inpyo Kim, Jeongju Park, Yong-Mu Kim, Hyunjung Shin, Jaegab Lee, and Frank Caruso. Layer-by-layer assembled chargetrap memory devices with adjustable electronic properties. Nature Nanotechnology, 2(12):790-795. 125

[164] Electrical bistability and charge transport behavior in Au nanoparticle/poly(Nvinylcarbazole) hybrid memory devices. Applied Physics Letters, 93(15):153305, 10 2008. 125

[165] V. Kannan, Y. S. Chae, Ch V. V. Ramana, Dong-Sik Ko, and J. K. Rhee. All-inorganic spin-cast quantum dot based bipolar nonvolatile resistive memory. Journal of Applied Physics, 109(8):086103, 4 2011. 125

[166] Jessamyn A. Fairfield, Tali Dadosh, and Marija Drndic. Characterization of memory and measurement history in photoconductivity of nanocrystal arrays. Applied Physics Letters, 97(14):143112, October 2010. 125

[167] Wen-Peng Lin, Shu-Juan Liu, Tao Gong, Qiang Zhao, and Wei Huang. PolymerBased Resistive Memory Materials and Devices. Advanced Materials, 26(4):570-606, 12014. 125 
[168] Qi-Dan Ling, Der-Jang Liaw, Chunxiang Zhu, Daniel Siu-Hung Chan, En-Tang Kang, and Koon-Gee Neoh. Polymer electronic memories: Materials, devices and mechanisms. Progress in Polymer Science, 33(10):917-978. 125

[169] Shuchao Qin, Ruixin Dong, Xunling Yan, and Qianqian Du. A reproducible write-(read)n-erase and multilevel bio-memristor based on DNA molecule. Organic Electronics, 22:147-153. 125

[170] B. C. Das, S. K. Batabyal, and A. J. Pal. A Bit per Particle: Electrostatic Assembly of CdSe Quantum Dots as Memory Elements. Advanced Materials, 19(23):4172-4176, 12 2007. 125

[171] Kallol Mohanta, Swarup K. Majee, Sudip K. Batabyal, and Amlan J. Pal. Electrical Bistability in Electrostatic Assemblies of CdSe Nanoparticles. The Journal of Physical Chemistry B, 110(37):18231-18235, 9 2006. 125

[172] Bikas C. Das and Amlan J. Pal. Memory Applications and Electrical Bistability of Semiconducting Nanoparticles: Do the Phenomena Depend on Bandgap? Small, 4(5):542-547, 5 2008. 125

[173] Batu Ghosh, Satyajit Sahu, and Amlan J. Pal. Core-Shell Nanoparticles: An Approach to Enhance Electrical Bistability. The Journal of Physical Chemistry C, 112(30):11290-11294, July 2008. 125

[174] Fushan Li, Dong Ick Son, Sung Hwan Cho, and Tae Whan Kim. Electrical bistabilities and operating mechanisms of memory devices fabricated utilizing $\mathrm{ZnO}$ quantum dot-multi-walled carbon nanotube nanocomposites. Nanotechnology, 20(18):185202. 125

[175] Timothy P. Osedach, Ni Zhao, Trisha L. Andrew, Patrick R. Brown, Darcy D. Wanger, David B. Strasfeld, Liang-Yi Chang, Moungi G. Bawendi, and Vladimir Bulović. Bias-Stress Effect in 1,2-Ethanedithiol-Treated PbS Quantum Dot FieldEffect Transistors. ACS Nano, 6(4):3121-3127, 4 2012. 125

[176] Yao Liu, Markelle Gibbs, James Puthussery, Steven Gaik, Rachelle Ihly, Hugh W. Hillhouse, and Matt Law. Dependence of Carrier Mobility on Nanocrystal Size and Ligand Length in PbSe Nanocrystal Solids. Nano Letters, 10(5):1960-1969, 52010. 125,141

[177] C. A. Leatherdale, C. R. Kagan, N. Y. Morgan, S. A. Empedocles, M. A. Kastner, and M. G. Bawendi. Photoconductivity in CdSe quantum dot solids. Physical Review B, 62(4):2669-2680, 7 2000. 125

[178] Deniz Bozyigit, Weyde M. M. Lin, Nuri Yazdani, Olesya Yarema, and Vanessa Wood. A quantitative model for charge carrier transport, trapping and recombination in nanocrystal-based solar cells. Nature Communications, 6:6180, 12015. 125

[179] Nicole Y. Morgan, C. A. Leatherdale, M. Drndić, Mirna V. Jarosz, Marc A. Kastner, and Moungi Bawendi. Electronic transport in films of colloidal CdSe nanocrystals. Physical Review B, 66(7):075339, 8 2002. 125 
[180] Marco Laurenti, Samuele Porro, Candido F. Pirri, Carlo Ricciardi, and Alessandro Chiolerio. Zinc Oxide Thin Films for Memristive Devices: A Review. Critical Reviews in Solid State and Materials Sciences, 42(2):153-172, 3 2017. 125

[181] Cong Ye, Jiaji Wu, Gang He, Jieqiong Zhang, Tengfei Deng, Pin He, and Hao Wang. Physical Mechanism and Performance Factors of Metal Oxide Based Resistive Switching Memory: A Review. Journal of Materials Science and Technology, 32(1):1-11. 125

[182] J. Joshua Yang, Feng Miao, Matthew D. Pickett, Douglas A. A. Ohlberg, Duncan R. Stewart, Chun Ning Lau, and R. Stanley Williams. The mechanism of electroforming of metal oxide memristive switches. Nanotechnology, 20(21):215201. 125, 149

[183] J. Joshua Yang, Matthew D. Pickett, Xuema Li, Douglas A. A. Ohlberg, Duncan R. Stewart, and R. Stanley Williams. Memristive switching mechanism for metal/oxide/metal nanodevices. Nature Nanotechnology, 3(7):429-433. 125, 149

[184] W. Szuszkiewicz. Optical absorption in Hg Te. physica status solidi (b), 79(2):691698, 2 1977. 132

[185] Optical Properties. In Physics and Properties of Narrow Gap Semiconductors, pages 239-383. Springer, New York, NY. DOI: 10.1007/978-0-387-74801-6. 132

[186] Scott Geyer, Venda J. Porter, Jonathan E. Halpert, Tamar S. Mentzel, Marc A. Kastner, and Moungi G. Bawendi. Charge transport in mixed CdSe and CdTe colloidal nanocrystal films. Physical Review B, 82(15):155201, 10 2010. 137

[187] Hyunsuk Kim, Kyoungah Cho, Dong-Won Kim, Hye-Ryoung Lee, and Sangsig Kim. Bottom- and top-gate field-effect thin-film transistors with $p$ channels of sintered HgTe nanocrystals. Applied Physics Letters, 89(17):173107, 2006. 140, 142

[188] Junggwon Yun, Kyoungah Cho, and Sangsig Kim. Flexible logic circuits composed of chalcogenide-nanocrystal-based thin film transistors. Nanotechnology, 21(23):235204, 2010. 140

[189] E. J. Meijer, D. M. de Leeuw, S. Setayesh, E. van Veenendaal, B.-H. Huisman, P. W. M. Blom, J. C. Hummelen, U. Scherf, and T. M. Klapwijk. Solution-processed ambipolar organic field-effect transistors and inverters. Nature Materials, 2(10):678682, October 2003. 140

[190] Dongyoon Khim, Eul-Yong Shin, Yong Xu, Won-Tae Park, Sung-Ho Jin, and YongYoung Noh. Control of Threshold Voltage for Top-Gated Ambipolar Field-Effect Transistor by Gate Buffer Layer. ACS Applied Materials \& Interfaces, 8(27):1741617420, July 2016. 141

[191] B. S. Sørensen, M. Aagesen, C. B. Sørensen, P. E. Lindelof, K. L. Martinez, and J. Nygård. Ambipolar transistor behavior in p-doped InAs nanowires grown by molecular beam epitaxy. Applied Physics Letters, 92(1):012119, January 2008. 141

[192] Yu Yamashita, Junto Tsurumi, Felix Hinkel, Yugo Okada, Junshi Soeda, Wojciech Zajaczkowski, Martin Baumgarten, Wojciech Pisula, Hiroyuki Matsui, Klaus Müllen, and Jun Takeya. Transition Between Band and Hopping Transport in Polymer Field-Effect Transistors. Advanced Materials, 26(48):8169-8173, December 2014. 142 
[193] J. C. Scott and L. D. Bozano. Nonvolatile Memory Elements Based on Organic Materials. Advanced Materials, 19(11):1452-1463, 6 2007. 145

[194] Ute Zschieschang, R. Thomas Weitz, Klaus Kern, and Hagen Klauk. Bias stress effect in low-voltage organic thin-film transistors. Applied Physics A, 95(1):139-145, 4 2009. 147

[195] Joseph M. Luther, Matt Law, Qing Song, Craig L. Perkins, Matthew C. Beard, and Arthur J. Nozik. Structural, Optical, and Electrical Properties of Self-Assembled Films of PbSe Nanocrystals Treated with 1,2-Ethanedithiol. ACS Nano, 2(2):271280, February 2008. 147

[196] Heng Liu, Alexandre Pourret, and Philippe Guyot-Sionnest. Mott and EfrosShklovskii Variable Range Hopping in CdSe Quantum Dots Films. ACS Nano, 4(9):5211-5216, 9 2010. 149

[197] Hugo E. Romero and Marija Drndic. Coulomb Blockade and Hopping Conduction in PbSe Quantum Dots. Physical Review Letters, 95(15):156801, 10 2005. 149, 218

[198] Lilei Hu, Andreas Mandelis, Zhenyu Yang, Xinxin Guo, Xinzheng Lan, Mengxia Liu, Grant Walters, Alexander Melnikov, and Edward H. Sargent. Temperatureand ligand-dependent carrier transport dynamics in photovoltaic PbS colloidal quantum dot thin films using diffusion-wave methods. Solar Energy Materials and Solar Cells, 164(Supplement C):135-145, 5 2017. 149

[199] Jianbo Gao and Justin C. Johnson. Charge Trapping in Bright and Dark States of Coupled PbS Quantum Dot Films. ACS Nano, 6(4):3292-3303, 4 2012. 149

[200] Fu-Chien Chiu. A Review on Conduction Mechanisms in Dielectric Films. Advances in Materials Science and Engineering, page 578168. DOI: 10.1155/2014/578168. 149

[201] D. S. Ginger and N. C. Greenham. Charge injection and transport in films of CdSe nanocrystals. Journal of Applied Physics, 87(3):1361-1368, 1 2000. 149

[202] Sean Keuleyan, Emmanuel Lhuillier, and Philippe Guyot-Sionnest. Synthesis of Colloidal HgTe Quantum Dots for Narrow Mid-IR Emission and Detection. Journal of the American Chemical Society, 133(41):16422-16424, 10 2011. 150

[203] Philippe Guyot-Sionnest and John Andris Roberts. Background limited midinfrared photodetection with photovoltaic HgTe colloidal quantum dots. Applied Physics Letters, 107(25):253104, 12 2015. 150

[204] Mengyu Chen, Hui Yu, Stephen V. Kershaw, Haihua Xu, Shuchi Gupta, Frederik Hetsch, Andrey L. Rogach, and Ni Zhao. Fast, Air-Stable Infrared Photodetectors based on Spray-Deposited Aqueous HgTe Quantum Dots. Advanced Functional Materials, 24(1):53-59, 12014.150

[205] Mengyu Chen, Haipeng Lu, Nema M. Abdelazim, Ye Zhu, Zhen Wang, Wei Ren, Stephen V. Kershaw, Andrey L. Rogach, and Ni Zhao. Mercury Telluride Quantum Dot Based Phototransistor Enabling High-Sensitivity Room-Temperature Photodetection at $2000 \mathrm{~nm}$. ACS Nano, 11(6):5614-5622, 6 2017. 150, 209 
[206] R. a. M. Hikmet, D. V. Talapin, and H. Weller. Study of conduction mechanism and electroluminescence in CdSe/ZnS quantum dot composites. Journal of Applied Physics, 93(6):3509-3514, 3 2003. 151

[207] Fatih Gul and Hasan Efeoglu. $\mathrm{ZnO}$ and $\mathrm{ZnO}_{1-x}$ based thin film memristors: The effects of oxygen deficiency and thickness in resistive switching behavior. Ceramics International, 43(14):10770-10775, 10 2017. 152

[208] Ruobing Pan, Jun Li, Fei Zhuge, Liqiang Zhu, Lingyan Liang, Hongliang Zhang, Junhua Gao, Hongtao Cao, Bing Fu, and Kang Li. Synaptic devices based on purely electronic memristors. Applied Physics Letters, 108(1):013504, 1 2016. 152

[209] Chih-Yang Lin, Sheng-Yi Wang, Dai-Ying Lee, and Tseung-Yuen Tseng. Electrical Properties and Fatigue Behaviors of $\mathrm{ZrO}_{2}$ Resistive Switching Thin Films. Journal of The Electrochemical Society, 155(8):H615-H619, 8 2008. 152

[210] Hyunsuk Kim, Kyoungah Cho, Dong-Won Kim, Hye-Ryoung Lee, and Sangsig Kim. Bottom- and top-gate field-effect thin-film transistors with $\mathrm{p}$ channels of sintered HgTe nanocrystals. Applied Physics Letters, 89(17):173107. 155

[211] As an honest means of properly illustrating the levels of performance that need to be surpassed once at the commercial stage, the author points the reader to https: //www.flir.com.au/products/flir-one-pro/. this is a microbolometer that offers a $160 \times 120$ pixel thermal sensor that operates in the 8-14 $\mu \mathrm{m}$ spectral range, has a pixel size of $12 \mu \mathrm{m}$, a frame rate of $8.7 \mathrm{~Hz}$ and can detect objects that are only $3{ }^{\circ} \mathrm{C}$ hotter than ambient temperature. this costs usd \$ 500 and can plug into an iphone. 162

[212] Amardeep Jagtap, Bertille Martinez, Nicolas Goubet, Audrey Chu, Clément Livache, Charlie Gréboval, Julien Ramade, Dylan Amelot, Paul Trousset, Amaury Triboulin, Sandrine Ithurria, Mathieu G. Silly, Benoit Dubertret, and Emmanuel Lhuillier. Design of a Unipolar Barrier for a Nanocrystal-Based Short-Wave Infrared Photodiode. ACS Photonics, 5(11):4569-4576, November 2018. 162

[213] Xin Tang, Matthew M. Ackerman, and Philippe Guyot-Sionnest. Thermal Imaging with Plasmon Resonance Enhanced HgTe Colloidal Quantum Dot Photovoltaic Devices. ACS Nano, 12(7):7362-7370, July 2018. 162, 169

[214] Matthew M. Ackerman, Xin Tang, and Philippe Guyot-Sionnest. Fast and Sensitive Colloidal Quantum Dot Mid-Wave Infrared Photodetectors. ACS Nano, 12(7):72647271, July 2018. 162, 169

[215] Matthew E. Cryer and Jonathan E. Halpert. $300 \mathrm{~nm}$ Spectral Resolution in the MidInfrared with Robust, High Responsivity Flexible Colloidal Quantum Dot Devices at Room Temperature. ACS Photonics, 5(8):3009-3015, August 2018. 163, 179

[216] Mengyu Chen, Hui Yu, Stephen V. Kershaw, Haihua Xu, Shuchi Gupta, Frederik Hetsch, Andrey L. Rogach, and Ni Zhao. Fast, Air-Stable Infrared Photodetectors based on Spray-Deposited Aqueous HgTe Quantum Dots. Advanced Functional Materials, 24(1):53-59, January 2014. 163, 179 
[217] Whi Dong Kim, Ji-Hee Kim, Sooho Lee, Seokwon Lee, Ju Young Woo, Kangha Lee, Weon-Sik Chae, Sohee Jeong, Wan Ki Bae, John A. McGuire, Jun Hyuk Moon, Mun Seok Jeong, and Doh C. Lee. Role of Surface States in Photocatalysis: Study of Chlorine-Passivated CdSe Nanocrystals for Photocatalytic Hydrogen Generation. Chemistry of Materials, 28(3):962-968, February 2016. 163

[218] Jong-Soo Lee, Maksym V. Kovalenko, Jing Huang, Dae Sung Chung, and Dmitri V. Talapin. Band-like transport, high electron mobility and high photoconductivity in all-inorganic nanocrystal arrays. Nature Nanotechnology, 6(6):348-352, June 2011. 177

[219] Hsiang-Yu Chen, Michael K. F. Lo, Guanwen Yang, Harold G. Monbouquette, and Yang Yang. Nanoparticle-assisted high photoconductive gain in composites of polymer and fullerene. Nature Nanotechnology, 3(9):543-547, September 2008. 177

[220] Gerasimos Konstantatos and Edward H. Sargent. PbS colloidal quantum dot photoconductive photodetectors: Transport, traps, and gain. Applied Physics Letters, 91(17):173505, October 2007. 177

[221] P Floyd, P Zarogiannis, M Crane, S Tarkowski, and V Bencko. Risks to Health and the Environment related to the use of Mercury Products. Technical report, The European Commission, August 2002. 185

[222] Sohrab Rabii. Energy-Band Structure and Electronic Properties of SnTe. Physical Review, 182(3):821-828, June 1969. 186

[223] Indika U. Arachchige and Mercouri G. Kanatzidis. Anomalous Band Gap Evolution from Band Inversion in $\mathrm{Pb}_{1-x} \mathrm{Sn}_{x}$ Te Nanocrystals. Nano Letters, 9(4):15831587, April 2009. 186, 191

[224] Maksym Yarema and Maksym V. Kovalenko. Colloidal Synthesis of InSb Nanocrystals with Controlled Polymorphism Using Indium and Antimony Amides. Chemistry of Materials, 25(9):1788-1792, May 2013. 186

[225] Jeffrey M. Pietryga, Richard D. Schaller, Donald Werder, Michael H. Stewart, Victor I. Klimov, and Jennifer A. Hollingsworth. Pushing the Band Gap Envelope: Mid-Infrared Emitting Colloidal PbSe Quantum Dots. Journal of the American Chemical Society, 126(38):11752-11753, September 2004. 186

[226] Maksym V. Kovalenko, Wolfgang Heiss, Elena V. Shevchenko, Jong-Soo Lee, Harald Schwinghammer, A. Paul Alivisatos, and Dmitri V. Talapin. SnTe Nanocrystals: A New Example of Narrow-Gap Semiconductor Quantum Dots. Journal of the American Chemical Society, 129(37):11354-11355, September 2007. 186, 191

[227] Youngjin Jang, Diana Yanover, Richard Karel Čapek, Arthur Shapiro, Nathan Grumbach, Yaron Kauffmann, Aldona Sashchiuk, and Efrat Lifshitz. Cation Exchange Combined with Kirkendall Effect in the Preparation of SnTe/CdTe and CdTe/SnTe Core/Shell Nanocrystals. The Journal of Physical Chemistry Letters, 7(13):2602-2609, July 2016. 186, 191, 210 
[228] Shaojun Guo, Andrew F. Fidler, Kai He, Dong Su, Gen Chen, Qianglu Lin, Jeffrey M. Pietryga, and Victor I. Klimov. Shape-Controlled Narrow-Gap SnTe Nanostructures: From Nanocubes to Nanorods and Nanowires. Journal of the American Chemical Society, 137(48):15074-15077, December 2015. 186, 191, 201, 210

[229] Ying Xu, Najeh Al-Salim, Justin M. Hodgkiss, and Richard D. Tilley. Solution Synthesis and Optical Properties of SnTe Nanocrystals. Crystal Growth \& Design, 11(7):2721-2723, July 2011. 186, 187, 191

[230] Ilka Kriegel, Jessica Rodríguez-Fernández, Andreas Wisnet, Hui Zhang, Christian Waurisch, Alexander Eychmüller, Aliaksei Dubavik, Alexander O. Govorov, and Jochen Feldmann. Shedding Light on Vacancy-Doped Copper Chalcogenides: Shape-Controlled Synthesis, Optical Properties, and Modeling of Copper Telluride Nanocrystals with Near-Infrared Plasmon Resonances. ACS Nano, 7(5):4367-4377, May 2013. 186

[231] Joseph M. Luther, Prashant K. Jain, Trevor Ewers, and A. Paul Alivisatos. Localized surface plasmon resonances arising from free carriers in doped quantum dots. Nature Materials, 10(5):361-366, May 2011. 186, 210

[232] Yang Liu, Maixian Liu, and Mark T. Swihart. Plasmonic Copper Sulfide-Based Materials: A Brief Introduction to Their Synthesis, Doping, Alloying, and Applications. The Journal of Physical Chemistry C, 121(25):13435-13447, June 2017. 186

[233] Xin Liu and Mark T. Swihart. Heavily-doped colloidal semiconductor and metal oxide nanocrystals: an emerging new class of plasmonic nanomaterials. Chemical Society Reviews, 43(11):3908-3920, 2014. 186

[234] Jacob A. Faucheaux, Alexandria L. D. Stanton, and Prashant K. Jain. Plasmon Resonances of Semiconductor Nanocrystals: Physical Principles and New Opportunities. The Journal of Physical Chemistry Letters, 5(6):976-985, March 2014. 186

[235] Fangyuan Lu, Juehan Yang, Renxiong Li, Nengjie Huo, Yongtao Li, Zhongming Wei, and Jingbo Li. Gas-dependent photoresponse of SnS nanoparticles-based photodetectors. Journal of Materials Chemistry C, 3(6):1397-1402, 2015. 186, 216

[236] A. Sa'ar, Y. Reichman, M. Dovrat, D. Krapf, J. Jedrzejewski, and I. Balberg. Resonant Coupling between Surface Vibrations and Electronic States in Silicon Nanocrystals at the Strong Confinement Regime. Nano Letters, 5(12):2443-2447, December 2005. 186, 213, 216

[237] Venkataramana Bonu, Arindam Das, S. Amirthapandian, Sandip Dhara, and Ashok Kumar Tyagi. Photoluminescence of oxygen vacancies and hydroxyl group surface functionalized $\mathrm{SnO}_{2}$ nanoparticles. Physical Chemistry Chemical Physics, 17(15):9794-9801, 2015. 186, 211, 212, 215

[238] M. Bettini and H. J. Richter. Oxidation in air and thermal desorption on $\mathrm{PbTe}$, SnTe and $\mathrm{Pb}_{0.8} \mathrm{sn}_{0.2}$ te Surfaces. Surface Science, 80:334-343, February 1979. 196, 211

[239] X-ray Photoelectron Spectroscopy (XPS) Reference Pages. 204

[240] Pavel Lukashev, Walter R. L. Lambrecht, Takao Kotani, and Mark van Schilfgaarde. Electronic and crystal structure of $\mathrm{Cu}_{2-x} \mathrm{~S}$ : Full-potential electronic structure calculations. Physical Review B, 76(19):195202, November 2007. 210 
[241] Mark J. Polking, Prashant K. Jain, Yehonadav Bekenstein, Uri Banin, Oded Millo, Ramamoorthy Ramesh, and A. Paul Alivisatos. Controlling Localized Surface Plasmon Resonances in GeTe Nanoparticles Using an Amorphous-to-Crystalline Phase Transition. Physical Review Letters, 111(3):037401, July 2013. 210

[242] Loredana Protesescu, Tanja Zünd, Maryna I. Bodnarchuk, and Maksym V. Kovalenko. Air-Stable, Near- to Mid-Infrared Emitting Solids of PbTe/CdTe Core-Shell Colloidal quantum dots. ChemPhysChem, 17(5):670-674, March 2016. 210,211

[243] Humaira Seema, K. Christian Kemp, Vimlesh Chandra, and Kwang S. Kim. Graphene- $\mathrm{SnO}_{2}$ composites for highly efficient photocatalytic degradation of methylene blue under sunlight. Nanotechnology, 23(35):355705, 2012. 211, 212

[244] N. M. Shaalan, D. Hamad, A. Y. Abdel-Latief, and M. A. Abdel-Rahim. Preparation of quantum size of tin oxide: Structural and physical characterization. Progress in Natural Science: Materials International, 26(2):145-151, April 2016. 211, 212

[245] D Amalric-Popescu and $\mathrm{F}$ Bozon-Verduraz. Infrared studies on $\mathrm{SnO}_{2}$ and $\mathrm{Pd} / \mathrm{SnO}_{2}$. Catalysis Today, 70(1):139-154, October 2001. 211, 212, 215

[246] S. Harbeck, A. Szatvanyi, N. Barsan, U. Weimar, and V. Hoffmann. DRIFT studies of thick film un-doped and Pd-doped $\mathrm{SnO}_{2}$ sensors: temperature changes effect and $\mathrm{CO}$ detection mechanism in the presence of water vapour. Thin Solid Films, 436(1):76-83, July 2003. 211, 212

[247] Matthias Batzill and Ulrike Diebold. The surface and materials science of tin oxide. Progress in Surface Science, 79(2):47-154, January 2005. 211, 216

[248] F. Hernandez-Ramirez, S. Barth, A. Tarancon, O. Casals, E. Pellicer, J. Rodriguez, A. Romano-Rodriguez, J. R. Morante, and S. Mathur. Water vapor detection with individual tin oxide nanowires. Nanotechnology, 18(42):424016, 2007. 211, 212, 213, 216

[249] N. Bârsan and U. Weimar. Understanding the fundamental principles of metal oxide based gas sensors; the example of $\mathrm{CO}$ sensing with $\mathrm{SnO}_{2}$ sensors in the presence of humidity. Journal of Physics: Condensed Matter, 15(20):R813, 2003. 211, 212, 213,216

[250] Soumen Das and V. Jayaraman. $\mathrm{SnO}_{2}$ : A comprehensive review on structures and gas sensors. Progress in Materials Science, 66:112-255, October 2014. 211

[251] Wenjin Wan, Yuehua Li, Xingping Ren, Yinping Zhao, Fan Gao, and Heyun Zhao. $2 \mathrm{~d} \mathrm{SnO}_{2}$ Nanosheets: Synthesis, Characterization, Structures, and Excellent Sensing Performance to Ethylene Glycol. Nanomaterials, 8(2):112, February 2018. 211

[252] G. Heiland and D. Kohl. Physical and Chemical Aspects of Oxidic Semiconductor Gas Sensors. In Tetsuro Seiyama, editor, Chemical Sensor Technology, Chemical Sensor Technology, pages 15-38. Elsevier, Amsterdam, 1988. 211, 212, 216

[253] Artem V. Marikutsa, Marina N. Rumyantseva, Lada V. Yashina, and Alexander M. Gaskov. Role of surface hydroxyl groups in promoting room temperature $\mathrm{CO}$ sensing by Pd-modified nanocrystalline $\mathrm{SnO}_{2}$. Journal of Solid State Chemistry, 183(10):2389-2399, October 2010. 212 
[254] Philip G. Harrison and Barry M. Maunders. Tin oxide surfaces. Part 12.-A comparison of the nature of tin(IV) oxide, tin(IV) oxide-silica and tin(IV) oxide-palladium oxide: surface hydroxyl groups and ammonia adsorption. Journal of the Chemical Society, Faraday Transactions 1: Physical Chemistry in Condensed Phases, 80(6):1341-1356, 1984. 215

[255] Tohru Azumi and Kazuo Matsuzaki. What Does the Term "Vibronic Coupling" Mean? Photochemistry and Photobiology, 25(3):315-326, March 1977. 216

[256] Ammar Zakar, Rihan Wu, Dimitri Chekulaev, Vera Zerova, Wei He, Leigh Canham, and Andrey Kaplan. Carrier dynamics and surface vibration-assisted Auger recombination in porous silicon. Physical Review B, 97(15):155203, April 2018. 216

[257] Stephen L. Weeks, Rohan P. Chaukulkar, Paul Stradins, and Sumit Agarwal. Photoluminescence behavior of plasma synthesized $\mathrm{Si}$ nanocrystals oxidized at low temperature in pure $\mathrm{O}_{2}$ and $\mathrm{H}_{2} \mathrm{O}$. Journal of Vacuum Science \& Technology A: Vacuum, Surfaces, and Films, 32(5):050604, August 2014. 216

[258] J. X. Wang, X. W. Sun, Y. Yang, H. Huang, Y. C. Lee, O. K. Tan, and L. Vayssieres. Hydrothermally grown oriented $\mathrm{ZnO}$ nanorod arrays for gas sensing applications. Nanotechnology, 17(19):4995, 2006. 216

[259] Zhiyong Fan and J. G. Lu. Chemical sensing with $\mathrm{ZnO}$ nanowire field-effect transistor. IEEE Transactions on Nanotechnology, 5(4):393-396, July 2006. 216

[260] Liwei Wang, Yanfei Kang, Xianghong Liu, Shoumin Zhang, Weiping Huang, and Shurong Wang. $\mathrm{ZnO}$ nanorod gas sensor for ethanol detection. Sensors and Actuators B: Chemical, 162(1):237-243, February 2012. 216

[261] SK Bera, S Chaudhuri, and AK Pal. Electron transport properties of cdte nanocrystals in $\mathrm{SiO}_{2} / \mathrm{CdTe} / \mathrm{SiO}_{2}$ thin film structures. Thin Solid Films, 415(1-2):68-77, 2002. 218

[262] Aarnoud L. Roest, John J. Kelly, and Daniël Vanmaekelbergh. Coulomb blockade of electron transport in a ZnO quantum-dot solid. Applied Physics Letters, 83(26):55305532, December 2003. 218

[263] A. Kolmakov, Y. Zhang, G. Cheng, and M. Moskovits. Detection of CO and O2 Using Tin Oxide Nanowire Sensors. Advanced Materials, 15(12):997-1000, June 2003. 219 


\section{Appendix A}

\section{Acronyms}

MIR Mid Infrared

NC Nanocrystal

QD Quantum Dot

HgTe Mercury Telluride

PbSnTe Lead Tin Telluride

IR Infrared

NIR Near Infrared

SWIR Short Wave Infrared

LWIR Long Wave Infrared

EHP Electron Hole Pair

PC Photoconductive

PV Photovoltaic

PN P-doped N-doped semiconductor junction 
MBE Molecular Beam Epitaxy

OFET Organic Field Effect Transistor

TFT Thin Film Transistor

FET Field Effect Transistor

$N_{C} \quad$ Number of carriers in conduction band

$N_{V} \quad$ Number of carriers in valence band

$V_{d} \quad$ Drain voltage

$V_{g} \quad$ Gate voltage

$V_{d s} \quad$ Drain-source voltage

$V_{T h} \quad$ Threshold voltage

CMOS Complementary metal-oxide-semiconductor

RAM Random Access Memory

DOS Density of States

CQD Colloidal Quantum Dot

CQDS Colloidal Quantum Dot Solid

VRH Variable Range Hopping

NNH Nearest Neighbour Hopping

MCT MerCad Telluride (HgCdTe)

FPA Focal Plan Array

QDIP Quantum Dot Infrared Photodetector

QWID Quantum Well Detector 
ETL Electron Transport Layer

HTL Hole Transport Layer

EDT Ethanedithiol

NW Nanowire

IDE Inter-digitated Elecrode

NEDT Net Equivalent Temperature Difference

ROIC Read Out Integrated Circuit

UV Ultraviolet

FTIR Fourier Transform Infrared Spectrometer

ATR Attenuated Total Reflectance (spectroscopy)

BB Blackbody

TEM Transmission Electron Microscopy

SAED Selective Area Electron Diffraction

EDS Energy Dispersive X-ray Spectroscopy (EDX)

STEM Scanning Transmission Electron Microscopy

SEM Scanning Electron Microscopy

XRD X-ray Diffraction

SMU Source Measurement Unit

PM Power Meter

PA Parameter Analyser

R Responsivity 
D* Specific Detectivity

PCB Printed Circuit Board

EQE External Quantum Efficiency

TOP Trioctylphosphine

DDT Dodecanethiol

OLA Oleylamine

EtOH Ethanol

PPA Propylamine

ODT Octadecanethiol

OA Oleic Acid

TCE Tetrachloroethylene

MeOH Methanol

DI Distilled (water)

PET Polyethylene Terepthalate

IPA Isopropyl Alcohol

PR Photoresist

FPS Frames per Second

NSD Noise Spectral Density

LSPR Localised Surface Plasmon Resonance

HRS High Resistance State

LRS Low Resistance State 
SAM Self Assembled Monolayer

APTMS (3-Aminopropyl)trimethoxysilane DUT Device Under Test 
Chapter A. Acronyms 


\section{Appendix B}

\section{Why Use Multiple Band Gaps?}

As was touched on in Chapters 1 and 3, one of the key advantages of CQDs is that they make it considerably easier to construct a transducer element that has more than one MIR bandgap. The clear follow up question to this is, how would multiple bandgaps at the sensor actually give extra information to a prospective user? Due to the spectral nature of thermal radiation this is a more complicated question than in the visible region. Conventional LWIR systems give an intensity reading that is proportional to the absorbed power between 8 and $14 \mu \mathrm{m}$, therefore it can be difficult to distinguish between, for example, a high emissivity colder object with a lower emissivity hotter object, as the emitted power per unit area of target may be similar. In contrast the simplest way to use multiple bandgaps (multicolour) is shown in Figure B.1 A, which shows the onset of spectral emission for the temperatures indicated. Consider a $2 \mu \mathrm{m}$ and a $3 \mu \mathrm{m}$ pixel, the $2 \mu \mathrm{m}$ pixel cannot see any of the temperatures shown, the $3 \mu \mathrm{m}$ can see (under perfect conditions) the $350 \mathrm{~K}$ and the $400 \mathrm{~K}$ BBs. Therefore no matter what intensity of flux is being detected, this arrangement of pixels can tell us if the target is hotter than $400 \mathrm{~K}$ (intensity information from both pixels), the target is between 400 and $300 \mathrm{~K}$ ( $2 \mu \mathrm{m}$ pixel is reading zero) or the target is colder than $300 \mathrm{~K}$ (both pixels are reading zero). Now clearly this argument is postulated on a perfect detector, for any real case 


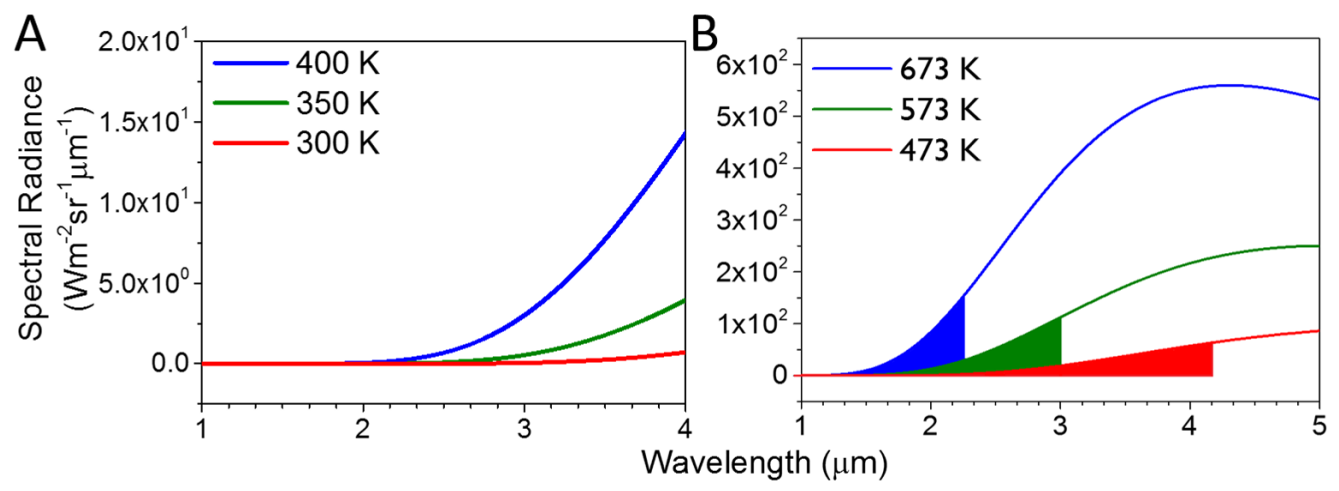

Figure B.1: $A$, wavelength region of the spectral onset of emission for the temperature BBs shown. B, spectral radiance of the the temperature BBs shown. The shaded areas represent the same area.

there will be some minimum detectable flux needed to cause a detectable signal. Figure B.1 B shows an example of this, where the shaded area is the same total radiance. The same argument as previously holds, but is modified as instead of looking at the spectral onset of emission, the requirement to be met for detection is that the total above bandgap radiance is above the minimum threshold. In this, hypothetical case, if the shaded area is the minimum detectable signal then, for a $3 \mu \mathrm{m}$ pixel the minimum detectable temperature is $573 \mathrm{~K}$. For a $4.2 \mu \mathrm{m}$ pixel the minimum detectable temperature is $473 \mathrm{~K}$. Using these limits one can again reconstruct temperature information, as well as intensity information. 